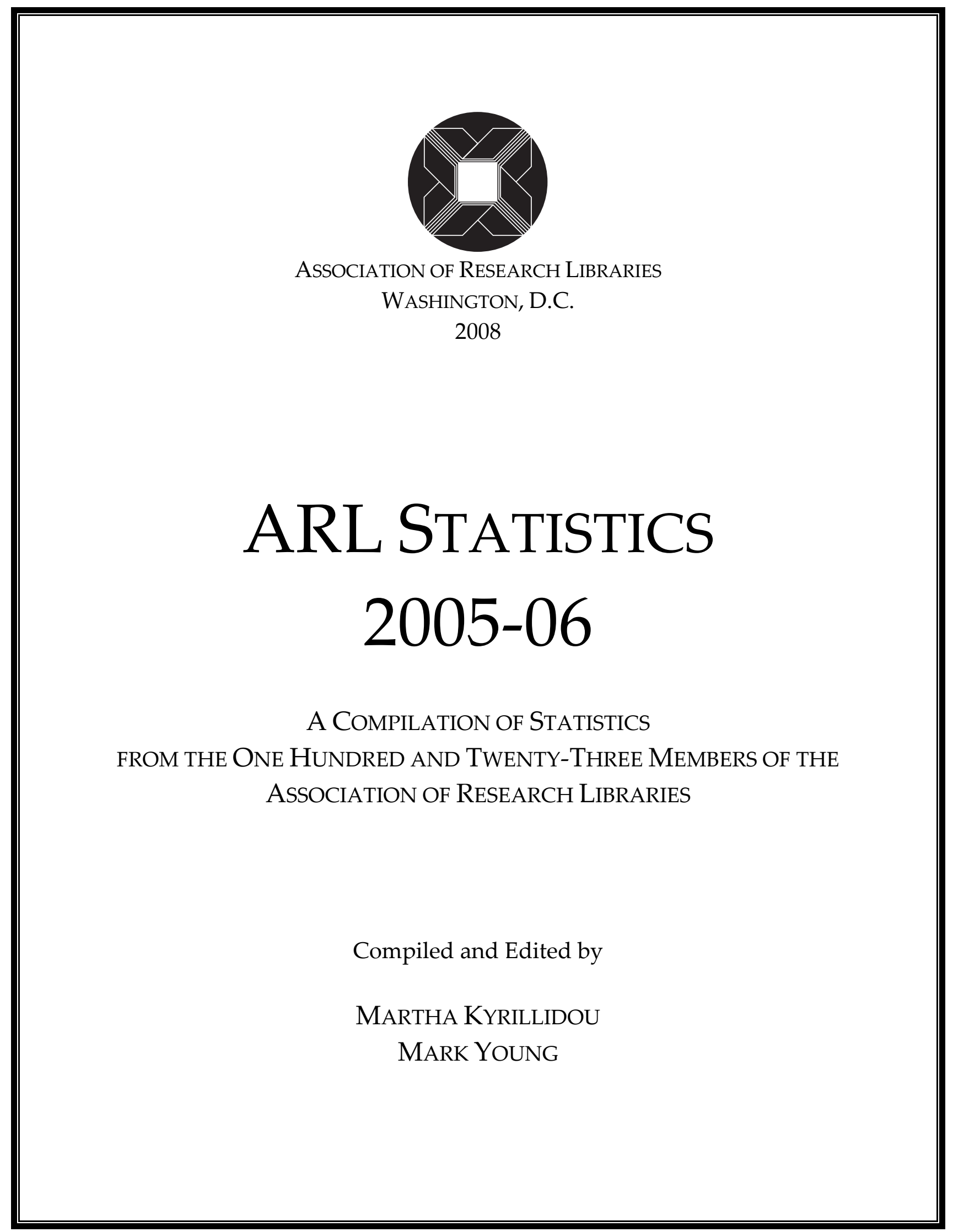


ARL Statistics is published annually by

\author{
Association of Research Libraries \\ 21 Dupont Circle, NW, Suite 800 \\ Washington, D.C. 20036 \\ Telephone: (202) 296-2296 \\ FAX: (202) 872-0884 \\ email: pubs@arl.org
}

\begin{abstract}
The ARL Statistics datafiles, with accompanying documentation, are available at http://www.arl.org/stats/
\end{abstract}

The tables presented in this publication are not indicative of performance and outcomes and should not be used as measures of library quality. In comparing any individual library to ARL medians or to other ARL members, one must be careful to make such comparisons within the context of differing institutional and local goals and characteristics.

ISBN 1-59407-785-1

EAN 9781594077852

(C) Copyright Notice

The compilation is copyrighted by the Association of Research Libraries. Blanket permission is granted to reproduce and distribute copies of this work for nonprofit, educational, or library purposes, provided that the author, source, and copyright notice are included on each copy. This permission is in addition to rights of reproduction granted under Sections 107, 108, and other provisions of the U.S. Copyright Act.

The paper used in this publication meets the minimum requirements of the American National Standard for Information Science and National Information Standards Organization standard-Permanence of Paper for Publications and Documents in Libraries and Archives, ANSI/NISO Z39.48-1992(R1997).

\title{
Printed in the United States of America
}




\section{TABLE OF CONTENTS}

Research Library Trends: An Introduction .....................................................................................

ARL Statistics Interactive Edition on the World Wide Web...............................................................25

ARL Library Data Tables, 2005-06

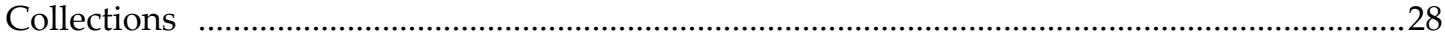

Summary Data

Expenditures

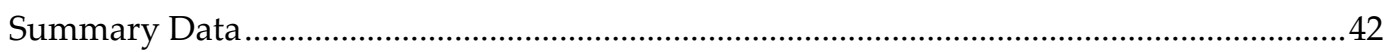

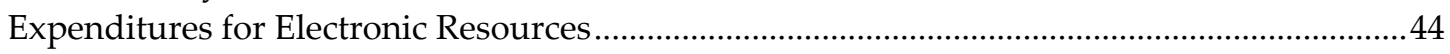

Summary Data

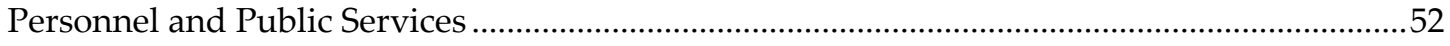

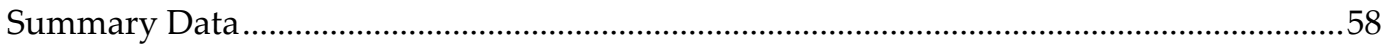

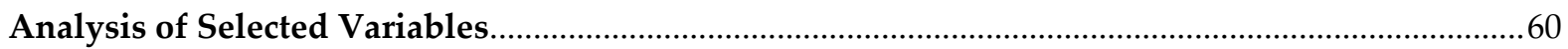

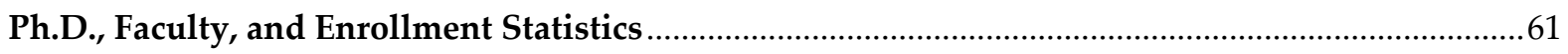

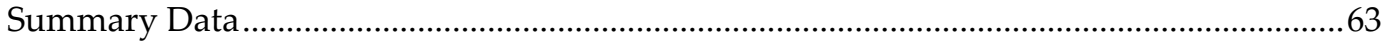

Rank Order Tables of University Libraries, 2005-06

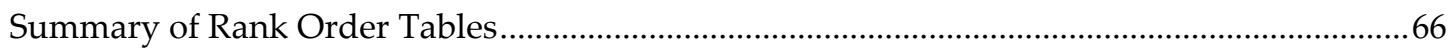

Individual Tables

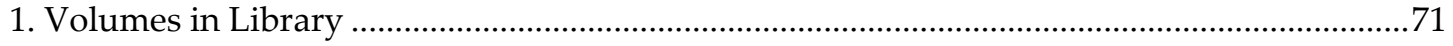

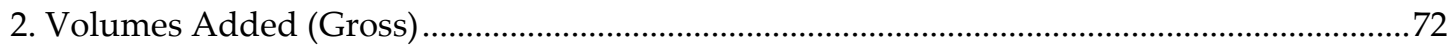

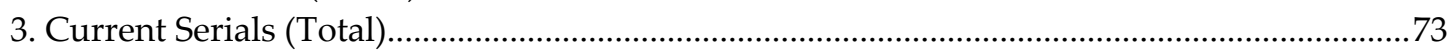

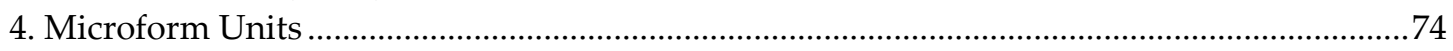

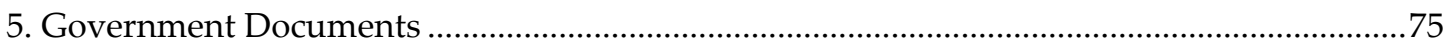

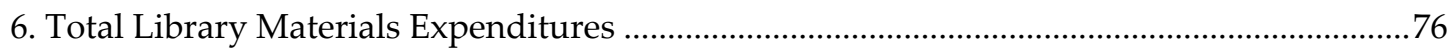

7. Total Salaries \& Wages Expenditures.....................................................................................7

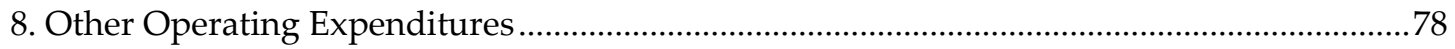

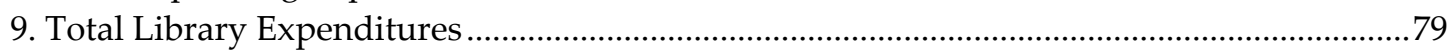

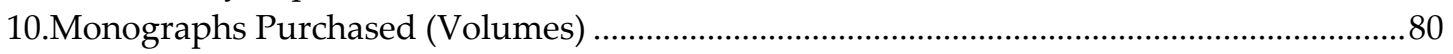

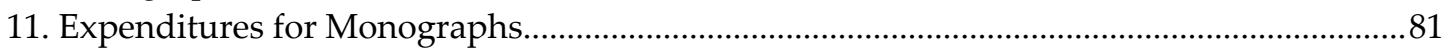

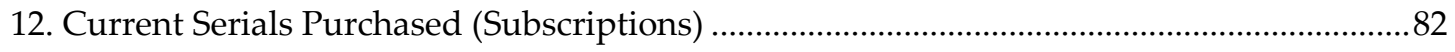

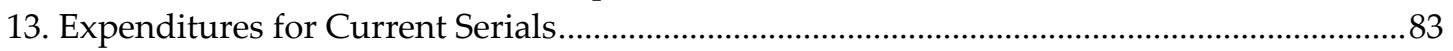

14. Total Items Loaned (ILL/DD) ………………

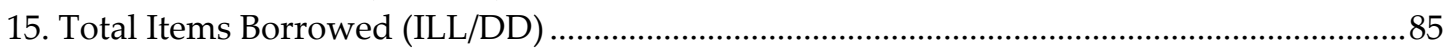

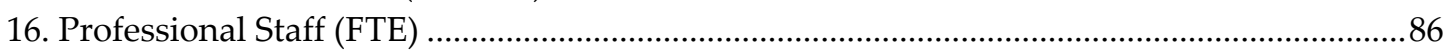

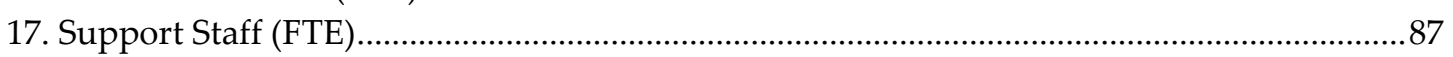

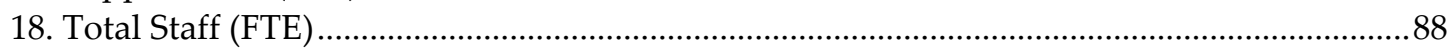

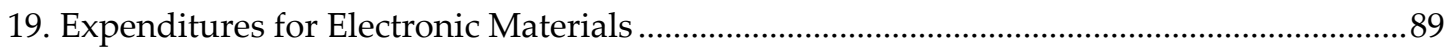

20. Electronic Materials as a Percent of Total Library Materials....................................................90 
ARL Statistics Questionnaire and Instructions, 2005-06 .93

Footnotes to the ARL Statistics 2005-06 107

Appendix A. ARL Member Libraries as of January 2008 .....................................................................130

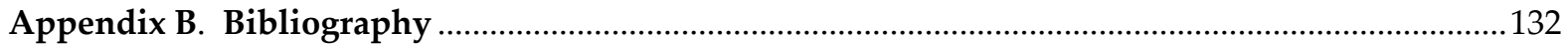

\section{GRAPHS}

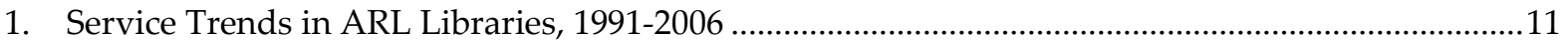

2. Monograph and Serial Costs in ARL Libraries, 1986-2006 ….......................................................13

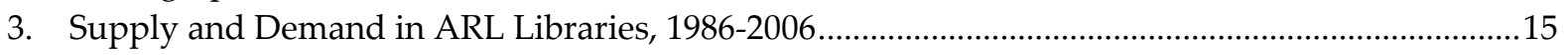

4. Expenditure Trends in ARL Libraries, 1986-2006 .........................................................................17

5. Resources per Student in ARL University Libraries, 1986-2006 .......................................................19

6. Yearly Increases in Average: Electronic Resources vs. Total Materials Expenditures, 1993-2006...21

\section{TABLES}

1. Service Trends in ARL Libraries, 1991-2006

2. Monograph and Serial Costs in ARL Libraries, 1986-2006 ….......................................................12

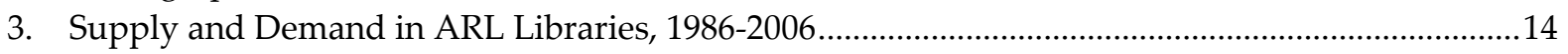

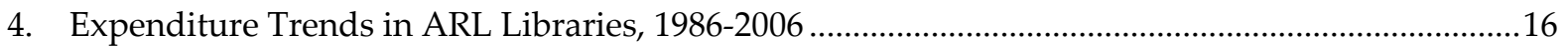

5. Resources per Student in ARL University Libraries, 1986-2006 .......................................................18

6. Electronic Materials Expenditures in ARL University Libraries, 2005-06 ........................................20

7a. Electronic Resources and Materials Expenditures in ARL University Libraries, 1992-2002 ............22

7b. Electronic Resources and Materials Expenditures in ARL University Libraries, 2002-06...............23 


\section{Reshaping ARL STATISTICS TO CAPTURE THE NEW ENVIRONMENT ${ }^{1}$}

The ARL Statistics 2005-06 describe a familiar picture for research libraries in North America. The rising cost of serials is outpacing general inflation, the cost of monographs is hovering close to inflation, and salaries are increasing moderately more quickly than inflation. As seen in Table 1 and Graph 1, the numbers of reference and circulation transactions have fallen from their levels of 10 years ago, but more users participated in instructional services offered by the library. Librarians are becoming more involved in the instructional process and are increasingly an integral part of the teaching and learning infrastructure at their institutions.

Historically, the perceived strength of a research library has been manifested in the size of its research collection-number of volumes held, volumes added, and serial subscriptions have been key indicators of quality as well as quantity in the eyes of some stakeholders. In a world where the basic unit of research information was the printed word, it was a plausible notion that the more you have of these things, the better equipped you may be in supporting high-quality research. One could argue that this is still the case. However, with the introduction of digital information and the dramatic changes in the nature of content, measuring the size of library collections cannot be what it used to be.

For example, in 2005-06 ARL libraries spent $43 \%$ of their materials budget on electronic resources - a total of $\$ 431$ million out of $\$ 1.1$ billion. This measure indicates the quantity and complexity that libraries are dealing with, but ultimately these figures cannot offer much when it comes to describing the quality of research, teaching, and learning at an institution. We need new measures to do this. ARL is beginning to address this need with important changes and additions to the ARL Statistics.

\section{From SERIAL SUbSCRIPTIONS TO SERIAL TITLES}

The unit cost of a serial subscription that ARL has tracked becomes relatively uninformative in a world where research libraries are increasingly offering access to the same serial title via multiple subscriptions and interfaces. The impact of electronic publishing on research library investment in serials was one of the forces behind a recommendation to move away from tracking serial subscriptions and towards tracking serial titles. ${ }^{5}$ The ARL Statistics 2005-06 is the last time ARL will publish a unit cost for serial subscriptions (see Table 2).

The ARL Statistics and Assessment Committee determined that a new way of counting serials based on titles rather than subscriptions would better reflect the true scope of the serial content provided by research libraries and recommended that ARL transform the serial counts from subscriptions into titles. With the revised definitions for survey year 2006-07, ARL libraries are now asked to report

\footnotetext{
${ }^{1}$ This article first appeared in ARL: A Bimonthly Report on Research Library Issues and Actions from ARL, CNI, and SPARC 256 (February 2008): 9-11.

${ }^{5}$ Martha Kyrillidou, "The Impact of Electronic Publishing in Tracking Research Library Investments in Serials," ARL: A Bimonthly Report, no. 249 (December 2006): 6-7, http://www.arl.org/bm doc/arlbr249serials.pdf.
} 
unduplicated counts of serial titles. Dual-format titles will be reported as electronic-only in the $A R L$ Statistics 2006-07, reflecting the current transition from print to electronic formats. ${ }^{6}$

The process of deriving serials title counts was tested over the period of a year and, although not perfect, it is feasible and practical in the short-term and much more meaningful in the long-term. ARL reported extensively on the testing done at Texas A\&M University and a detailed process for implementation is documented in the ARL Statistics Webcast, which is available on the ARL Web site. ${ }^{7}$ Issues have emerged related to ISSN standardization practices, serials with no ISSN assignments, branch and department libraries that are independent from one another and hard to deduplicate, and difficulties regarding the implementation of new procedures. Collaborative discussion on how to address such issues is taking place through postings on the Library Assessment Blog8 and during in-person conversations at ARL Survey Coordinators workshops and meetings. ARL also provides an ARL Statistics FAQ online to help member libraries move into the new paradigm of counting serials. ${ }^{9}$

\section{FROM COLLECTIONS TO EXPENDITURES}

In an environment where collections are morphing into terabytes, petabytes, exabytes, zettabytes, and yottabytes of information, it is questionable whether the units of volumes held, volumes added, and serial subscriptions can continue to offer the utility they had in the past. As shown in Table 3 and Graph 3, it is now much more common for items to be loaned and lended between libraries than to be purchased.

The challenge of measuring collections in new ways gave rise to the work of the ARL Task Force on New Ways of Measuring Collections. ${ }^{10}$ During its two-year investigation, the task force systematically collected qualitative feedback through one-on-one interviews with each ARL library director and, during the second year of its operation, the task force deployed two top researchers in qualitative and quantitative methodologies, Yvonna Lincoln and Bruce Thompson. Two reports were produced for the ARL community: "Research Libraries as Knowledge Producers: A Shifting Context for Policy and Funding,"11 documenting the results of the qualitative inquiry, and "Some Alternative Quantitative Library Activity Descriptions/Statistics that Supplement the ARL Logarithmic Index,"12 documenting the results of the quantitative inquiry. Based on these reports, the task force forwarded a set of recommendations to the ARL Board of Directors that formed the following action agenda for the ARL Statistics and Assessment Committee during 2007:

1. Reserve use of the current Membership Criteria Index for those occasions when it is needed for consideration of membership issues.

2. Implement an Expenditures-Focused Index.

\footnotetext{
${ }^{6}$ Richard K. Johnson and Judy Luther, "The E-Only Tipping Point for Journals" (Washington DC: ARL, 2007), http://www.arl.org/bm doc/Electronic_Transition.pdf; Karla Hahn, "The State of the Large Publisher Bundle: Findings from an ARL Member Survey," ARL: A Bimonthly Report, no. 245 (April 2006): 1-6,

http:/ / www.arl.org/bm doc/arlbr245bundle.pdf.

${ }^{7}$ ARL Statistics Webcast, http://www.arl.org/arldocs/stats/statsevents/stats_webcast/120407ARL_final.html.

${ }^{8}$ Library Assessment Blog, http:/ / libraryassessment.info/?cat=28.

${ }^{9}$ ARL Statistics FAQ, http://www.arl.org/bm doc/statsfaq_dec3.pdf.

${ }^{10}$ Task Force on New Ways of Measuring Collections, http://www.arl.org/stats/aboutstats/tfnewways.shtml.

${ }^{11}$ Yvonna Lincoln, "Research Libraries as Knowledge Producers: A Shifting Context for Policy and Funding" (Washington DC: ARL, 2006), http://www.arl.org/bm doc/lincoln.pdf.

${ }^{12}$ Bruce Thompson, "Some Alternative Quantitative Library Activity Descriptions/Statistics that Supplement the ARL

Logarithmic Index" (Washington DC: ARL, 2006), http://www.arl.org/bm doc/bruce_3mk.pdf.
} 
3. Use the new Expenditures-Focused Index for any public reports, such as in the Chronicle of Higher Education.

4. Begin to develop a Services-Based Index that combines the following three factors: collections, services, and collaborative relationships.

5. Revise definitions for collections-related data categories currently collected and experiment with a variety of new measures, including usage data, strength of collections, and service quality measures to develop a richer set of variables for potential inclusion in the three-factor ServicesBased Index (see above).

6. Collect qualitative data to develop a profile of ARL member libraries.

The issue of fluctuating rankings in the ARL Membership Criteria Index previously published in the Chronicle of Higher Education gave rise to the systematic investigation of the nature of the five variables included in this Index: volumes held, volumes added (gross), serial subscriptions, total expenditures, and professional staff plus support staff. Through the quantitative analysis performed on the existing variables, alternative approaches were proposed. The first implementation was the development of an ARL Expenditures-Focused Index composed of four variables: total expenditures, expenditures for library materials, expenditures for professional salaries, and total professional staff plus support staff. As can be seen in Table 4 and Graph 4, the expenditures variables are much less prone to "spikes" in any given survey year, compared to the serial data shown in Graph 2.

Thus, for the first time this year, the Chronicle of Higher Education published the new ARL Expenditures-Focused Index. The Expenditures-Focused Index calculates principal component scores and the analysis is based on all university member libraries' data (as compared with the Membership Criteria Index, which is based on the 34 founding members of the Association). The ExpendituresFocused Index is a summary measure of relative size of the investment made by ARL university members' parent institutions in their libraries. It has been calculated retrospectively beginning with data from 2002-03.14

Although similar to the ARL Membership Criteria Index in reflecting the investments made in research libraries, the ARL Expenditures-Focused Index is less affected by the rapidly changing context of library collections.

\section{DEVELOPING NEW INDICATORS}

The new Expenditures-Focused Index is only the first step in reshaping the ARL Statistics. ARL's historical descriptive statistics are being re-examined and adjusted to reflect this changing context of collection access and ownership.

The ARL Statistics and Assessment Committee is currently engaged in developing new quantitative and qualitative indicators and indices to describe research library collections and services and their contribution to research, teaching, and learning. In particular, the notion of a three-factor index describing collections, services, and collaborative relations is a viable proposed construct as tested with the existing variables. Yet more work is needed to develop robust variables that withstand the passing of time and allow us to describe the nature of libraries into the future. For example, collaborative relations currently are being measured with two variables: interlibrary borrowing and lending. Other new ways of

\footnotetext{
${ }^{14}$ Expenditures-Focused Index, http://www.arl.org/stats/index/.
} 
sharing information like consortial purchasing, collaborative remote storage, and collaborative purchasing have emerged. We have a challenge in how we measure such concepts.

Key aspects of collaborative relations may be described only in qualitative terms in the future. For example, in the recently published commemorative volume of ARL's $75^{\text {th }}$ anniversary, Celebrating Research, the editors include library overviews and profiles for the rare and special collections available for use in research libraries. ${ }^{15}$ The ARL Statistics and Assessment Committee members are moving forward with the challenge of constructing profiles for entire research libraries and rendering them as succinct descriptions that will be analyzed to determine the elements of standardized ways for measuring research libraries in both qualitative and quantitative terms.

The complexity of research libraries in the digital future is hard for us to capture in the beginning of the $21^{\text {st }}$ century. We seek to define new ways for describing research libraries that will have the enduring value that has historically characterized the ARL Statistics.

${ }^{15}$ Philip N. Cronenwett, Kevin Osborn, Samuel A. Streit, eds., Celebrating Research: Rare and Special Collections from the Membership of the Association of Research Libraries (Washington DC: ARL, 2007), http:/ / www.celebratingresearch.org/. 


\section{RESEARCH LIBRARY TRENDS}

ARL Statistics 2005-06 is the latest in a series of annual publications that describe collections, staffing, expenditures, and service activities for the 123 members of the Association of Research Libraries (ARL). Of these, 113 are university libraries; the remaining 10 are public, governmental, and nonprofit research libraries. ARL member libraries are the largest research libraries in North America, representing 16 Canadian and 107 U.S. research institutions. The academic libraries, which comprise about $92 \%$ of the membership, include 14 Canadian and 99 U.S. libraries.

Statistics have been collected and published annually for the members of the Association of Research Libraries since 1961-62, and the data are available through an interactive Web interface. Prior to 1961-62, annual statistics for university libraries were collected by James Gerould, first at the University of Minnesota and later at Princeton University. ${ }^{16}$ These data, covering the years 1907-08 through 1961-62, are now called the Gerould statistics. ${ }^{17}$ The whole data series from 1908, which is available on the ARL FTP server, ${ }^{18}$ represents the oldest and most comprehensive continuing library statistical series in North America.

ARL libraries are a relatively small subset of libraries in North America, but they do account for a large portion of academic library resources in terms of assets, budgets, and the number of users they serve. The total library expenditures of all 123 member libraries in 2005-06 was more than $\$ 3.7$ billion; from that, roughly $\$ 2.84$ billion was spent by the 113 university libraries and more than $\$ 866$ million by the nonuniversity libraries. The pie charts below show how the two types of libraries divide these expenditures differently.

\section{University Libraries}

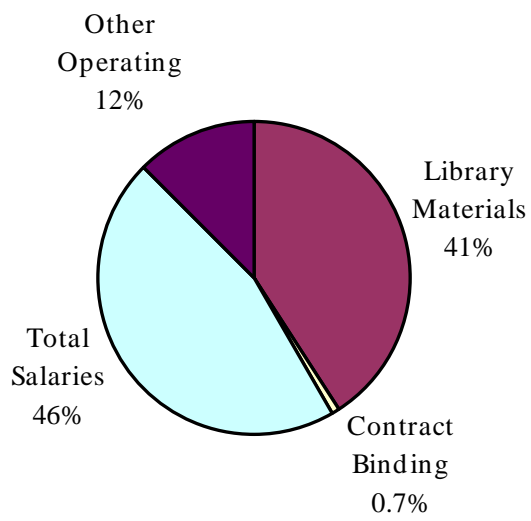

Nonuniversity Libraries

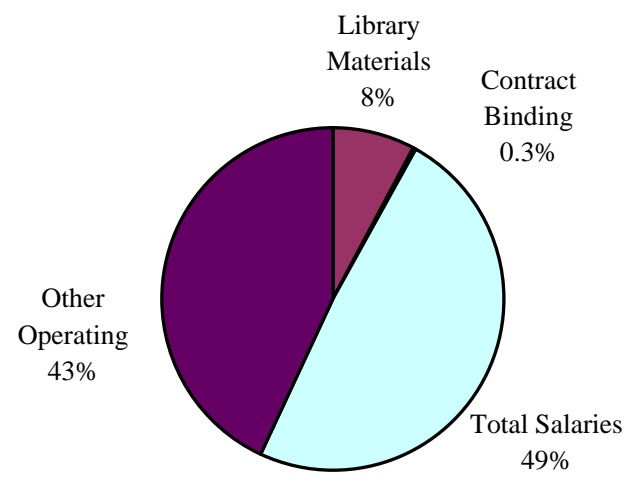

${ }^{16}$ Kendon L. Stubbs and Robert E. Molyneux, Research Library Statistics 1907-08 through 1987-88 (Washington, DC: ARL, 1990).

${ }^{17}$ Robert E. Molyneux, The Gerould Statistics 1907/08 - 1961/62. (Washington, DC: ARL, 1986),

http://fisher.lib.virginia.edu/gerould/.

${ }^{18} \mathrm{http}: / /$ www.arl.org/stats/arlstat/mrstat.html. 
Table 1

Service Trends in ARL Libraries, 1991-2006

Median Values for Time-Series Trends

\begin{tabular}{lrrrrrrrrr}
\hline Year & $\begin{array}{r}\text { ILL: } \\
\text { Borrowed }\end{array}$ & $\begin{array}{r}\text { Group } \\
\text { Pres. }\end{array}$ & $\begin{array}{r}\text { Participants } \\
\text { In Pres. }\end{array}$ & $\begin{array}{r}\text { Reference } \\
\text { Trans. }{ }^{19}\end{array}$ & $\begin{array}{r}\text { Initial } \\
\text { Circ. }\end{array}$ & $\begin{array}{r}\text { Total } \\
\text { Circ. }\end{array}$ & $\begin{array}{r}\text { Ratio of } \\
\text { Init./Tot. }\end{array}$ & $\begin{array}{r}\text { Total } \\
\text { Staff }\end{array}$ & $\begin{array}{r}\text { Total } \\
\text { Students }\end{array}$ \\
\hline (Libraries) & $(103)$ & $(84)$ & $(82)$ & $(79)$ & $(36)$ & $(80)$ & $(34)$ & $(105)$ & $(103)$ \\
1991 & 10,397 & 508 & 7,137 & 125,103 & 296,964 & 509,673 & 1.26 & 271 & 18,290 \\
1992 & 11,362 & 526 & 7,154 & 132,549 & 342,989 & 554,579 & 1.27 & 265 & 18,273 \\
1993 & 12,489 & 616 & 7,688 & 136,115 & 343,293 & 568,628 & 1.32 & 262 & 18,450 \\
1994 & 14,007 & 568 & 7,831 & 147,582 & 369,996 & 572,749 & 1.31 & 264 & 18,305 \\
1995 & 14,472 & 687 & 8,461 & 147,023 & 347,144 & 578,989 & 1.32 & 267 & 18,209 \\
1996 & 15,278 & 719 & 8,410 & 155,336 & 336,481 & 560,244 & 1.39 & 264 & 18,320 \\
1997 & 16,264 & 687 & 9,218 & 149,659 & 348,157 & 542,438 & 1.37 & 273 & 18,166 \\
1998 & 17,656 & 698 & 9,462 & 132,850 & 354,924 & 514,574 & 1.37 & 273 & 18,335 \\
1999 & 18,942 & 711 & 9,406 & 128,696 & 300,923 & 514,087 & 1.38 & 277 & 18,609 \\
2000 & 20,475 & 722 & 9,596 & 115,636 & 273,231 & 482,542 & 1.42 & 267 & 18,908 \\
2001 & 21,902 & 669 & 10,121 & 104,409 & 265,195 & 467,277 & 1.48 & 269 & 19,102 \\
2002 & 21,339 & 776 & 11,350 & 95,910 & 251,146 & 462,223 & 1.51 & 279 & 19,925 \\
2003 & 22,146 & 806 & 12,516 & 89,150 & 248,689 & 479,733 & 1.57 & 277 & 21,132 \\
2004 & 25,737 & 757 & 12,864 & 84,546 & 261,526 & 496,369 & 1.60 & 273 & 21,562 \\
2005 & 25,729 & 803 & 13,782 & 65,168 & 250,971 & 473,216 & 1.58 & 267 & 22,047 \\
2006 & 27,412 & 833 & 13,051 & 67,697 & 267,213 & 466,403 & 1.52 & 267 & 22,618 \\
\hline Avg annual & $6.2 \%$ & $3.1 \%$ & $3.8 \%$ & $-3.8 \%$ & $-0.7 \%$ & $-0.6 \%$ & $1.2 \%$ & $-0.1 \%$ & $1.3 \%$ \\
$\%$ change & & & & & & & & \\
\hline
\end{tabular}

Table 1 presents data about select public service activities such as circulation (initial and total), reference transactions, library instruction (group presentations and participants in these presentations), and interlibrary borrowing and lending.

As seen in Graph 1, for the third time in the last five years, the median of group presentations increased to a record high, with 833 in 2005-06. Meanwhile, the number of participants in those presentations has leveled off at just over 13,000 participants for the typical research library. Perhaps of most interest that by 2006 , about $45 \%$ of the presentations conducted in a typical ARL library had been added since 1991.

\footnotetext{
19 Time series for Reference Transactions revised due to unavailable data.
} 


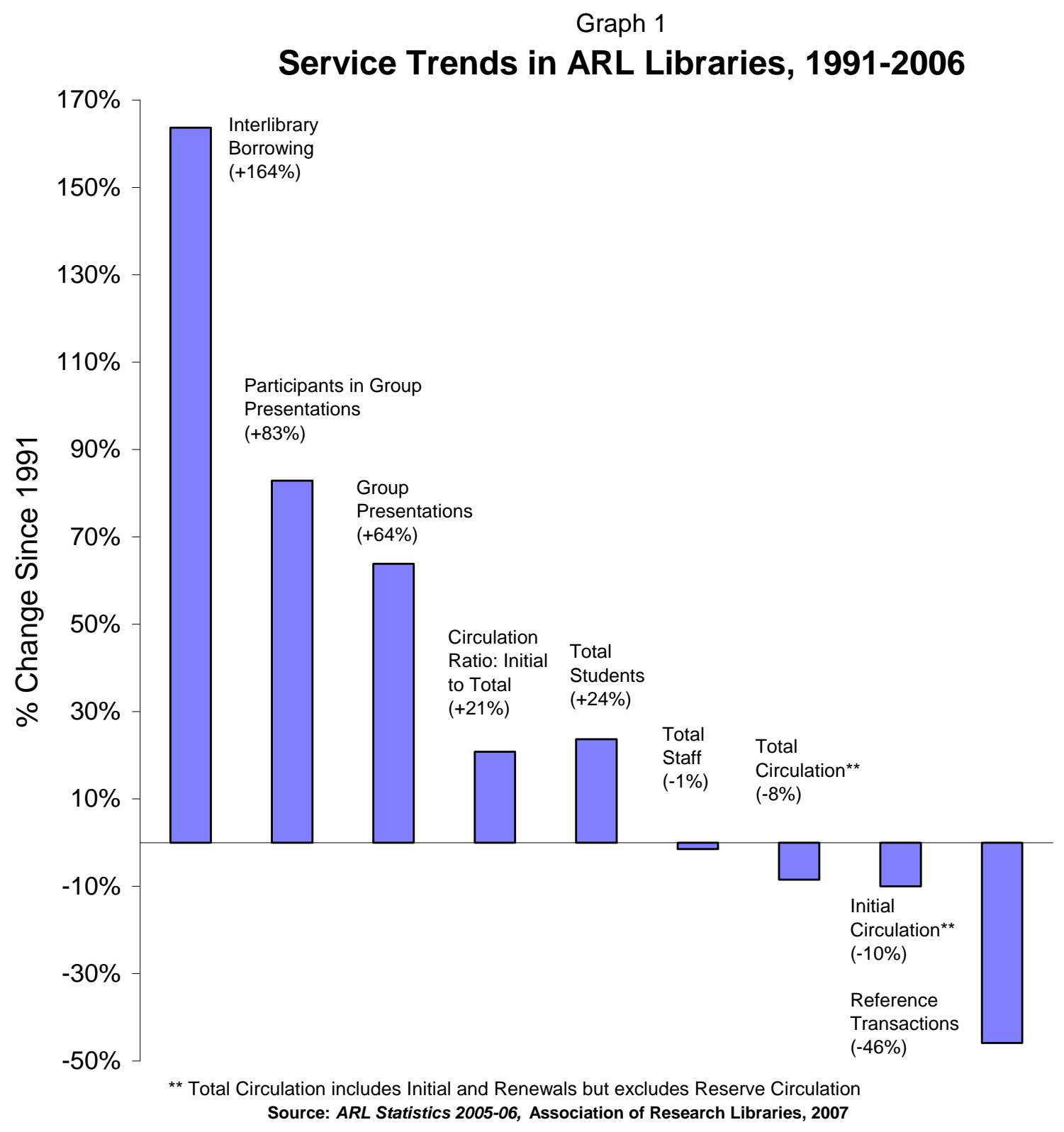


Table 2

Median Monograph and Serial Costs in ARL Libraries, 1986-2006 Median Values for Time-Series Trends ${ }^{20}$

\begin{tabular}{|c|c|c|c|c|c|c|c|}
\hline \multirow{2}{*}{$\begin{array}{l}\text { Year } \\
\text { (Libraries) }\end{array}$} & \multirow{2}{*}{$\begin{array}{r}\begin{array}{r}\text { Serial } \\
\text { Unit Cost }\end{array} \\
(36)\end{array}$} & \multirow{2}{*}{$\begin{array}{r}\begin{array}{r}\text { Serial } \\
\text { Expenditures }\end{array} \\
(100)\end{array}$} & \multicolumn{2}{|c|}{$\begin{array}{r}\text { Monograph } \\
\text { Unit Cost }\end{array}$} & \multirow{2}{*}{$\begin{array}{r}\begin{array}{r}\text { Monograph } \\
\text { Expenditures }\end{array} \\
(97)\end{array}$} & \multirow{2}{*}{$\begin{array}{r}\begin{array}{r}\text { Serials } \\
\text { Purchased }\end{array} \\
(36)\end{array}$} & \multirow{2}{*}{$\begin{array}{r}\begin{array}{r}\text { Monographs } \\
\text { Purchased }\end{array} \\
(58)\end{array}$} \\
\hline & & & & (57) & & & \\
\hline 1986 & $\$ 89.81$ & $\$ 1,496,775$ & $\$$ & 29.28 & $\$ 1,120,645$ & 15,775 & 32,679 \\
\hline 1987 & $\$ 108.12$ & $\$ 1,769,960$ & $\$$ & 31.76 & $\$ 1,064,484$ & 16,514 & 26,240 \\
\hline 1988 & $\$ 117.41$ & $\$ 1,947,559$ & $\$$ & 36.03 & $\$ 1,141,226$ & 15,948 & 25,238 \\
\hline 1989 & $\$ 129.95$ & $\$ 2,113,976$ & $\$$ & 38.44 & $\$ 1,106,073$ & 15,983 & 27,082 \\
\hline 1990 & $\$ 135.61$ & $\$ 2,296,910$ & $\$$ & 41.44 & $\$ 1,330,747$ & 16,128 & 27,546 \\
\hline 1991 & $\$ 153.46$ & $\$ 2,548,687$ & $\$$ & 42.60 & $\$ 1,400,738$ & 15,962 & 27,843 \\
\hline 1992 & $\$ 173.69$ & $\$ 2,620,832$ & $\$$ & 44.14 & $\$ 1,353,865$ & 15,673 & 26,947 \\
\hline 1993 & $\$ 188.79$ & $\$ 2,918,569$ & $\$$ & 43.74 & $\$ 1,295,807$ & 15,441 & 25,188 \\
\hline 1994 & $\$ 203.87$ & $\$ 2,912,495$ & $\$$ & 44.72 & $\$ 1,309,807$ & 15,099 & 25,341 \\
\hline 1995 & $\$ 217.38$ & $\$ 3,131,033$ & $\$$ & 45.27 & $\$ 1,365,575$ & 14,320 & 25,707 \\
\hline 1996 & $\$ 223.98$ & $\$ 3,389,118$ & $\$$ & 46.73 & $\$ 1,444,015$ & 14,723 & 25,911 \\
\hline 1997 & $\$ 250.74$ & $\$ 3,642,541$ & $\$$ & 46.42 & $\$ 1,460,234$ & 14,820 & 28,576 \\
\hline 1998 & $\$ 252.28$ & $\$ 3,816,497$ & $\$$ & 47.59 & $\$ 1,486,764$ & 14,063 & 24,447 \\
\hline 1999 & $\$ 271.51$ & $\$ 4,095,934$ & $\$$ & 47.78 & $\$ 1,506,651$ & 14,192 & 25,173 \\
\hline 2000 & $\$ 310.62$ & $\$ 4,430,812$ & $\$$ & 47.87 & $\$ 1,657,349$ & 14,541 & 28,024 \\
\hline 2001 & $\$ 279.07$ & $\$ 4,660,349$ & $\$$ & 48.48 & $\$ 1,864,023$ & 13,682 & 30,494 \\
\hline 2002 & $\$ 289.84$ & $\$ 4,939,225$ & $\$$ & 50.61 & $\$ 1,812,826$ & 17,594 & 31,578 \\
\hline 2003 & $\$ 282.20$ & $\$ 5,392,007$ & $\$$ & 52.83 & $\$ 1,873,157$ & 18,115 & 33,208 \\
\hline 2004 & $\$ 256.01$ & $\$ 5,580,157$ & $\$$ & 51.36 & $\$ 1,839,412$ & 22,311 & 30,155 \\
\hline 2005 & $\$ 239.58$ & $\$ 5,962,446$ & $\$$ & 53.57 & $\$ 1,784,841$ & 22,404 & 30,546 \\
\hline 2006 & \$251.38 & $\$ 6,307,292$ & $\$$ & 52.04 & $\$ 2,044,272$ & 23,849 & 33,145 \\
\hline $\begin{array}{l}\text { Avg annual } \\
\% \text { change }\end{array}$ & $5.3 \%$ & $7.5 \%$ & & $2.9 \%$ & $3.1 \%$ & $2.1 \%$ & $0.1 \%$ \\
\hline
\end{tabular}

Serial unit costs have been increasing much faster than inflation for almost two decades, as is detailed in Table 2 and Graph 2. For the third year in a row, the unit cost of serials declined and more and more serial subscriptions became available to libraries.

In informal conversations held with some ARL libraries, it seems that the inclusion of electronic serials in the counts of serial subscriptions purchased caused an increase in purchased serials - often, for a relatively small addition to the base subscription price, some publishers provide access to electronic resources for an additional 10 or $20 \%$ surcharge. Such arrangements played a role in the revision of the serial-counting practices in the ARL Statistics (see "From Serial Subscriptions to Serial Titles," above).

\footnotetext{
${ }^{20}$ Time series for Serials Expenditures, Monograph Expenditures, Monographs Purchased, and Monograph Unit cost were revised due to unavailable data.
} 
Graph 2

\section{Monograph and Serial Expenditures in ARL Libraries, 1986-2006*}

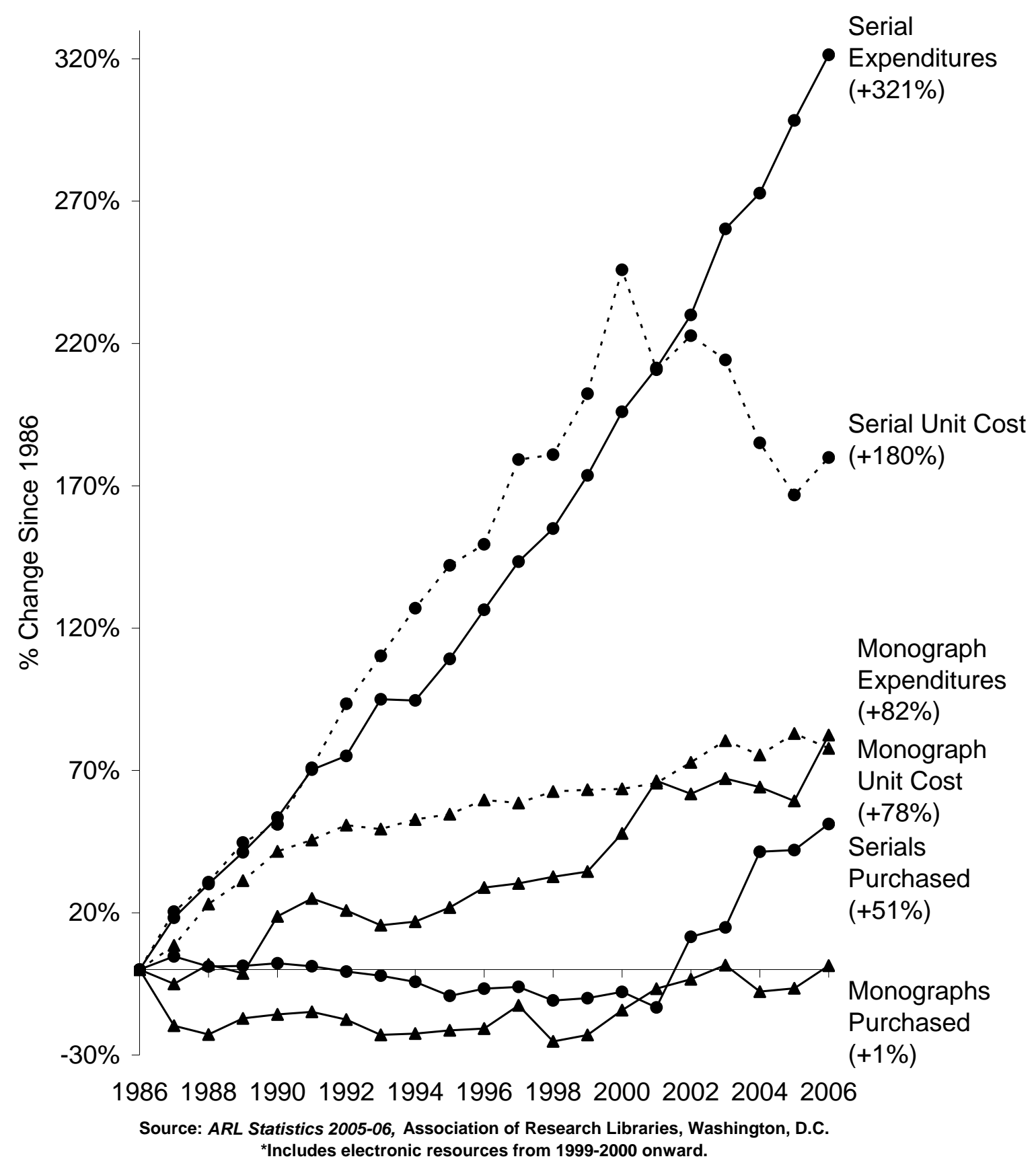


Table 3

Supply and Demand in ARL Libraries, 1986-2006 Median Values for Time-Series Trends ${ }^{21}$

\begin{tabular}{lrrrrrrrr}
\hline Year & $\begin{array}{r}\text { ILL: } \\
\text { Borrowed }\end{array}$ & $\begin{array}{r}\text { Lended } \\
\text { Lended }\end{array}$ & $\begin{array}{r}\text { Graduate } \\
\text { Students }\end{array}$ & $\begin{array}{r}\text { Teaching } \\
\text { Faculty }\end{array}$ & $\begin{array}{r}\text { Total } \\
\text { Students }\end{array}$ & $\begin{array}{r}\text { Serials } \\
\text { Purchased }\end{array}$ & $\begin{array}{r}\text { Serials } \\
\text { Received }\end{array}$ & $\begin{array}{r}\text { Monographs } \\
\text { Purchased }\end{array}$ \\
\hline (Libraries) & $(103)$ & $(103)$ & $(104)$ & $(101)$ & $(103)$ & $(36)$ & $(36)$ & $(58)$ \\
1986 & & & & & & & & \\
1987 & 7,047 & 16,092 & 2,327 & 1,124 & 16,684 & 15,775 & 3,318 & 32,679 \\
1988 & 7,387 & 16,318 & 3,078 & 1,195 & 17,029 & 16,514 & 3,477 & 26,240 \\
1989 & 7,881 & 17,476 & 3,251 & 1,222 & 17,485 & 15,948 & 3,367 & 25,238 \\
1990 & 8,547 & 19,638 & 3,312 & 1,285 & 17,866 & 15,983 & 3,345 & 27,082 \\
1991 & 9,595 & 20,837 & 3,314 & 1,278 & 17,745 & 16,128 & 4,304 & 27,546 \\
1992 & 10,397 & 23,285 & 3,310 & 1,295 & 18,290 & 15,962 & 4,500 & 27,843 \\
1993 & 11,362 & 22,514 & 3,539 & 1,356 & 18,273 & 15,673 & 5,100 & 26,947 \\
1994 & 12,489 & 22,740 & 3,745 & 1,281 & 18,450 & 15,441 & 5,082 & 25,188 \\
1995 & 14,007 & 24,039 & 3,794 & 1,289 & 18,305 & 15,099 & 5,518 & 25,341 \\
1996 & 14,472 & 24,864 & 3,914 & 1,308 & 18,209 & 14,320 & 6,107 & 25,707 \\
1997 & 15,278 & 25,720 & 3,904 & 1,251 & 18,320 & 14,723 & 5,983 & 25,911 \\
1998 & 16,264 & 25,463 & 3,942 & 1,263 & 18,166 & 14,820 & 5,757 & 28,576 \\
1999 & 17,656 & 27,223 & 3,880 & 1,247 & 18,335 & 14,063 & 7,111 & 24,447 \\
2000 & 18,942 & 26,837 & 3,933 & 1,255 & 18,609 & 14,192 & 6,546 & 25,173 \\
2001 & 20,475 & 27,044 & 3,844 & 1,239 & 18,908 & 14,541 & 7,944 & 28,024 \\
2002 & 21,902 & 28,950 & 4,159 & 1,279 & 19,102 & 13,682 & 7,915 & 30,494 \\
2003 & 21,339 & 29,021 & 4,067 & 1,251 & 19,925 & 17,594 & 8,769 & 31,578 \\
2004 & 22,146 & 33,421 & 4,167 & 1,268 & 21,132 & 18,115 & 8,871 & 33,208 \\
2005 & 25,737 & 33,934 & 4,461 & 1,369 & 21,562 & 22,311 & 9,991 & 30,155 \\
2006 & 25,729 & 36,325 & 4,595 & 1,355 & 22,047 & 22,404 & 11,203 & 30,546 \\
\hline & 27,412 & 36,035 & 4,647 & 1,388 & 22,618 & 23,849 & 11,980 & 33,145 \\
\hline Avg annual & & & & & & & & \\
\hline change & $7.0 \%$ & $4.1 \%$ & $3.5 \%$ & $1.1 \%$ & $1.5 \%$ & $2.1 \%$ & $6.6 \%$ & $0.1 \%$ \\
\hline
\end{tabular}

Table 3 and Graph 3 show that the number of non-purchased serials received by the average ARL library increased by an annual average of $6.6 \%$ since 1986 . This category consists of a number of types of serials, including government documents, electronic serials made available free of charge with the purchase of print counterparts, and open access journals. Interlibrary borrowing and lending, which showed a marked growth in the last decade, seem to be stabilizing.

In 2005-06 the median subscriptions reached a new high in the history of the time series, and monographs purchase rose above 1986 levels for the second time in the last nineteen years.

\footnotetext{
${ }^{21}$ Time series for Monographs Purchased were revised due to unavailable data.
} 


\section{Graph 3 \\ Supply and Demand in ARL Libraries, 1986-2006*}

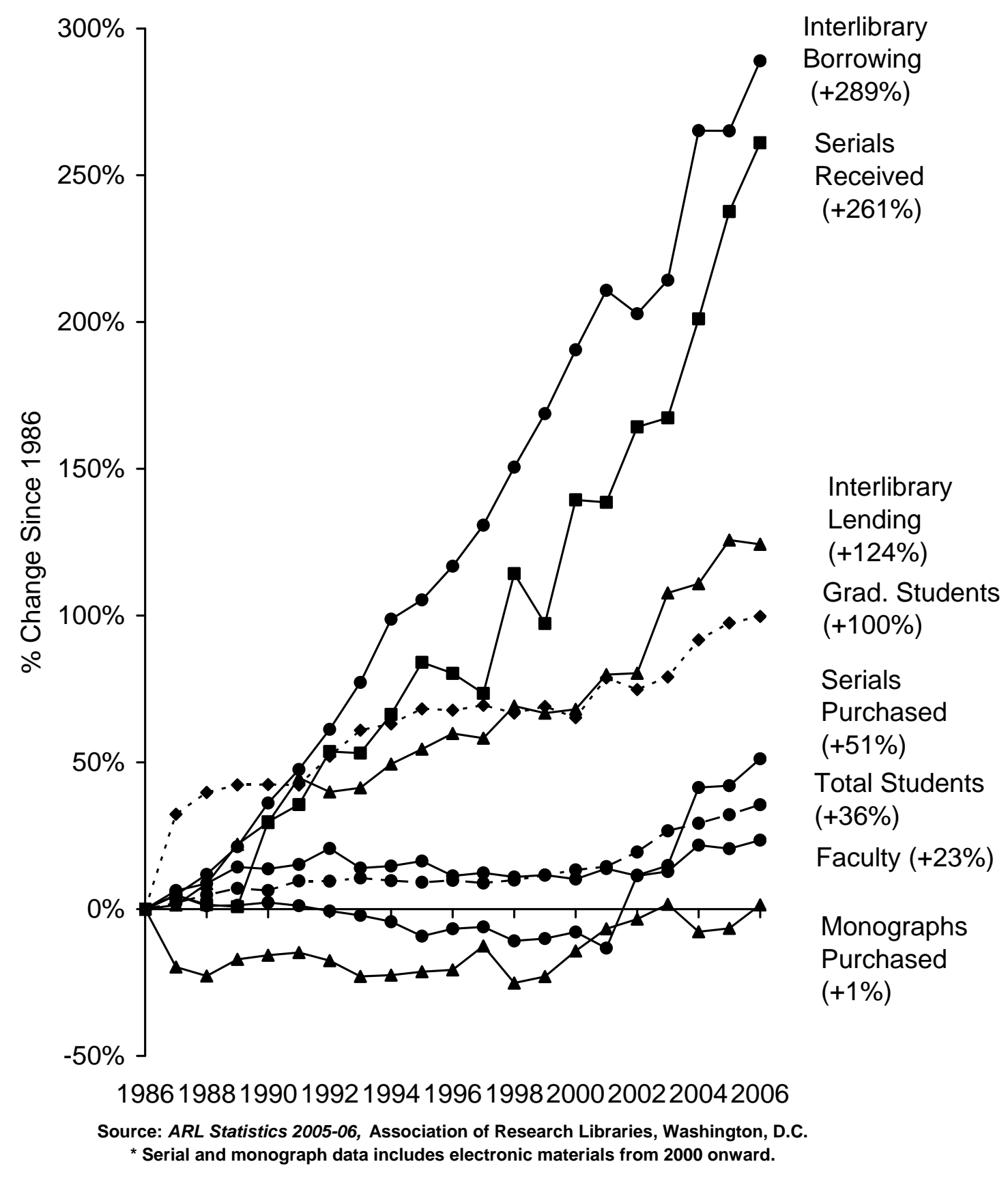


Table 4

Expenditure Trends in ARL Libraries, 1986-2006 Median Values for Time-Series Trends - Unadjusted dollar figures ${ }^{22}$

\begin{tabular}{|c|c|c|c|c|c|c|c|}
\hline Year & $\begin{array}{r}\text { Library } \\
\text { Materials }\end{array}$ & $\begin{array}{l}\text { Serial } \\
\text { Exp. }\end{array}$ & $\begin{array}{r}\text { Monograph } \\
\text { Exp. }\end{array}$ & $\begin{array}{r}\text { Total } \\
\text { Salary }\end{array}$ & $\begin{array}{r}\text { Operating } \\
\text { Exp. }\end{array}$ & Total & CPI \\
\hline (Libraries) & (105) & (100) & (97) & (104) & (104) & (105) & \\
\hline 1986 & 2,705,297 & $1,496,775$ & $1,120,645$ & $4,011,436$ & $1,111,914$ & $8,361,092$ & 109.6 \\
\hline 1987 & $3,058,479$ & $1,769,960$ & $1,064,484$ & $4,361,646$ & $1,180,167$ & $8,990,001$ & 113.6 \\
\hline 1988 & $3,369,896$ & $1,947,559$ & $1,141,226$ & $4,618,335$ & $1,198,674$ & $9,557,623$ & 118.3 \\
\hline 1989 & $3,577,405$ & $2,113,976$ & $1,106,073$ & $5,236,292$ & $1,364,558$ & $10,183,315$ & 124.0 \\
\hline 1990 & $3,903,358$ & $2,296,910$ & $1,330,747$ & $5,469,333$ & $1,386,618$ & $11,241,022$ & 130.7 \\
\hline 1991 & $4,064,344$ & $2,548,687$ & $1,400,738$ & $5,885,814$ & $1,445,735$ & $11,990,794$ & 136.2 \\
\hline 1992 & $4,156,510$ & $2,620,832$ & $1,353,865$ & $6,050,222$ & $1,390,245$ & $12,249,150$ & 140.3 \\
\hline 1993 & $4,316,674$ & $2,918,569$ & $1,295,807$ & $5,962,470$ & $1,561,122$ & $12,265,696$ & 144.5 \\
\hline 1994 & $4,572,276$ & $2,912,495$ & $1,309,807$ & $6,047,803$ & $1,676,701$ & $12,767,348$ & 148.2 \\
\hline 1995 & $4,715,203$ & $3,131,033$ & $1,365,575$ & $6,312,770$ & $1,853,586$ & $13,171,893$ & 152.4 \\
\hline 1996 & $5,126,482$ & $3,389,118$ & $1,444,015$ & $6,664,021$ & $1,997,233$ & $13,870,378$ & 156.9 \\
\hline 1997 & $5,562,742$ & $3,642,541$ & $1,460,234$ & $6,893,582$ & $2,039,957$ & $14,526,674$ & 160.5 \\
\hline 1998 & $5,795,223$ & $3,816,497$ & $1,486,764$ & $7,163,979$ & $2,072,903$ & $15,329,371$ & 163.0 \\
\hline 1999 & $6,232,365$ & $4,095,934$ & $1,506,651$ & $7,476,532$ & $2,069,887$ & $16,737,261$ & 166.6 \\
\hline 2000 & $6,744,281$ & $4,430,812$ & $1,657,349$ & $7,811,403$ & $1,991,852$ & $17,221,441$ & 172.2 \\
\hline 2001 & $7,322,507$ & $4,660,349$ & $1,864,023$ & $8,106,666$ & $2,280,493$ & $17,620,048$ & 177.5 \\
\hline 2002 & $7,599,249$ & $4,939,225$ & $1,812,826$ & $8,488,255$ & $2,136,616$ & $18,456,038$ & 180.1 \\
\hline 2003 & $8,273,171$ & $5,392,007$ & $1,873,157$ & $8,813,191$ & $2,073,913$ & $19,030,188$ & 183.9 \\
\hline 2004 & $8,286,431$ & $5,580,157$ & $1,839,412$ & $9,015,741$ & $2,274,878$ & $19,953,776$ & 189.4 \\
\hline 2005 & $8,801,962$ & $5,962,446$ & $1,784,841$ & $9,268,364$ & $2,243,592$ & $20,663,012$ & 195.4 \\
\hline 2006 & $9,174,734$ & $6,307,292$ & $2,044,272$ & $9,647,695$ & $2,540,171$ & $21,694,210$ & 203.5 \\
\hline $\begin{array}{l}\text { Avg annual } \\
\% \text { change }\end{array}$ & $6.3 \%$ & $7.5 \%$ & $3.1 \%$ & $4.5 \%$ & $4.2 \%$ & $4.9 \%$ & $3.1 \%$ \\
\hline
\end{tabular}

Operating expenditures, including many automation expenditures, have doubled since 1986, but during the last five years have remained at relatively stable levels of investment. The total salary expenditures median has increased only slightly over the past four years. Monograph expenditures continue to increase. The annual consumer price index (CPI), included in Graph 4, provides a comparative reference for the increases in library expenditures.

\footnotetext{
22 Time series for Serial Expenditures and Monograph Expenditures were revised due to unavailable data.
} 
Graph 4

Expenditure Trends

in ARL Libraries, 1986-2006

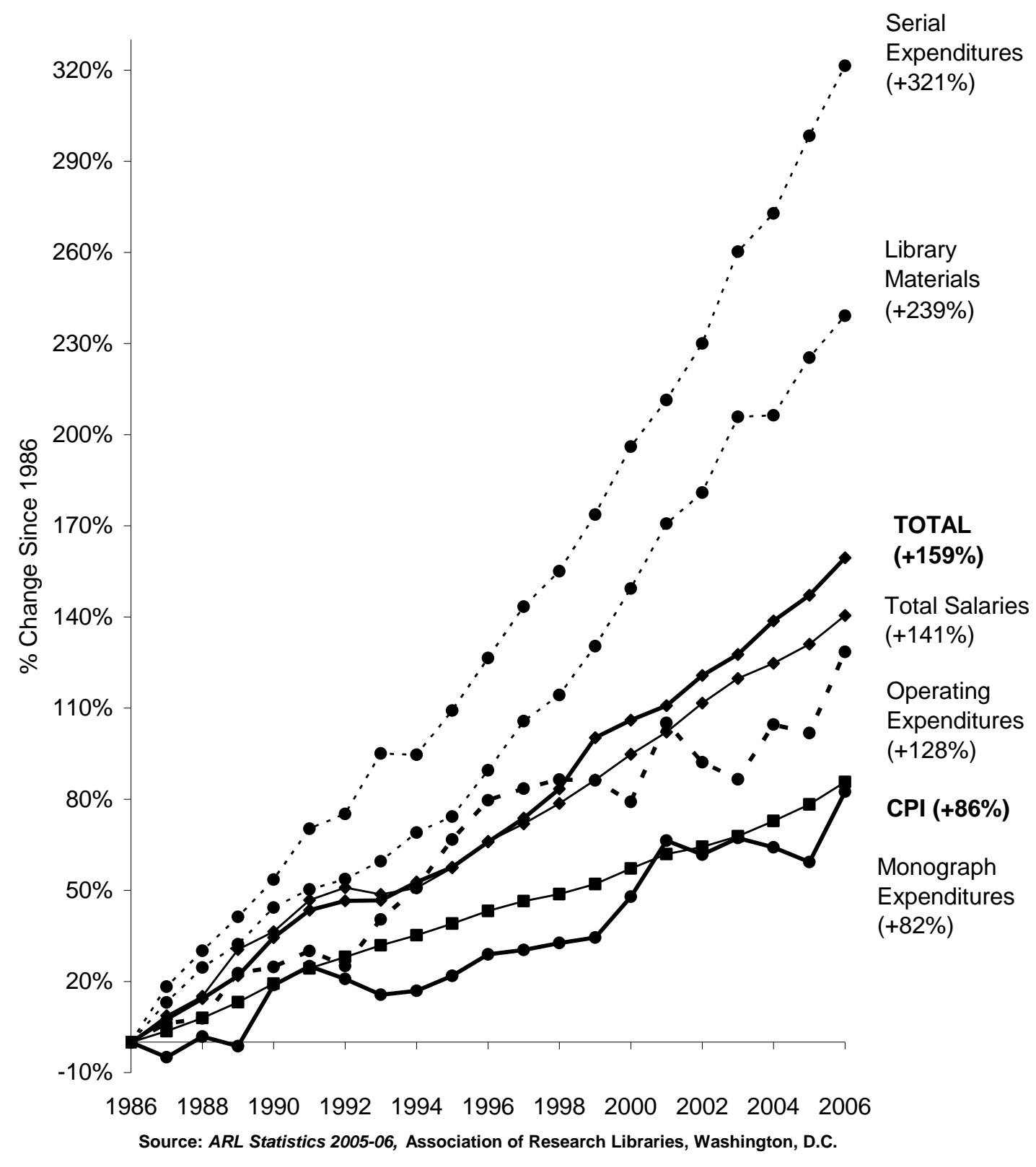


TABLE 5

Resources per Student in ARL University Libraries, 1986-2006 Median of Ratio Values for Time-Series Trends ${ }^{23}$

\begin{tabular}{|c|c|c|c|c|c|c|}
\hline Year & $\begin{array}{r}\text { ILL: } \\
\text { Borrowed }\end{array}$ & $\begin{array}{r}\text { Volumes } \\
\text { Held }\end{array}$ & $\begin{array}{r}\text { Volumes } \\
\text { Added } \\
\text { (Gross) }\end{array}$ & $\begin{array}{l}\text { Total } \\
\text { Staff }\end{array}$ & $\begin{array}{r}\text { Serials } \\
\text { Purchased }\end{array}$ & $\begin{array}{r}\text { Monographs } \\
\text { Purchased }\end{array}$ \\
\hline (Libraries) & (102) & (103) & (103) & (103) & (36) & (57) \\
\hline 1986 & 0.42 & 123.18 & 4.17 & 0.0159 & 0.85 & 2.20 \\
\hline 1987 & 0.44 & 125.31 & 3.92 & 0.0164 & 0.84 & 1.88 \\
\hline 1988 & 0.44 & 128.27 & 3.82 & 0.0161 & 0.85 & 1.66 \\
\hline 1989 & 0.50 & 130.04 & 3.84 & 0.0162 & 0.86 & 1.77 \\
\hline 1990 & 0.55 & 133.51 & 3.86 & 0.0155 & 0.86 & 1.83 \\
\hline 1991 & 0.63 & 137.11 & 4.04 & 0.0154 & 0.85 & 1.86 \\
\hline 1992 & 0.62 & 137.68 & 3.95 & 0.0153 & 0.84 & 1.58 \\
\hline 1993 & 0.68 & 146.02 & 3.76 & 0.0152 & 0.80 & 1.49 \\
\hline 1994 & 0.74 & 151.20 & 4.07 & 0.0152 & 0.79 & 1.55 \\
\hline 1995 & 0.80 & 154.00 & 4.37 & 0.0147 & 0.83 & 1.59 \\
\hline 1996 & 0.89 & 158.10 & 3.78 & 0.0145 & 0.80 & 1.48 \\
\hline 1997 & 0.89 & 159.40 & 4.03 & 0.0142 & 0.77 & 1.52 \\
\hline 1998 & 0.97 & 159.24 & 4.06 & 0.0143 & 0.73 & 1.44 \\
\hline 1999 & 1.05 & 159.37 & 4.00 & 0.0142 & 0.77 & 1.42 \\
\hline 2000 & 1.13 & 157.74 & 3.82 & 0.0139 & 0.77 & 1.63 \\
\hline 2001 & 1.16 & 155.71 & 3.89 & 0.0136 & 0.79 & 1.69 \\
\hline 2002 & 1.14 & 155.04 & 3.87 & 0.0131 & 0.82 & 1.54 \\
\hline 2003 & 1.14 & 156.41 & 3.52 & 0.0125 & 0.85 & 1.47 \\
\hline 2004 & 1.20 & 151.49 & 3.45 & 0.0120 & 0.98 & 1.53 \\
\hline 2005 & 1.22 & 157.93 & 3.30 & 0.0118 & 1.10 & 1.42 \\
\hline 2006 & 1.22 & 160.05 & 3.26 & 0.0117 & 1.08 & 1.64 \\
\hline $\begin{array}{l}\text { Avg annual } \\
\% \text { change }\end{array}$ & $5.4 \%$ & $1.3 \%$ & $-1.2 \%$ & $-1.5 \%$ & $1.2 \%$ & $-1.4 \%$ \\
\hline
\end{tabular}

Table 5 and Graph 5 show that per-student borrowing activity through interlibrary loan has continued to increase, and libraries are borrowing three times more items on a per-student basis than they did 20 years ago. ${ }^{24}$ Additionally, over the 20-year period since 1986, ARL libraries are acquiring $25 \%$ fewer monographs per student, but $27 \%$ more serials. Libraries also reported fewer staff per student in 2006 as compared to 1986: in 2006, there was a median number of 11.7 total staff per 1,000 students, compared to the 1986 figure of 16 per 1,000.

${ }^{23}$ All time-series in this table have been revised due to unavailable data.

${ }^{24}$ This overall trend should not be interpreted rigidly, as it negates the varying experiences of individual libraries. 


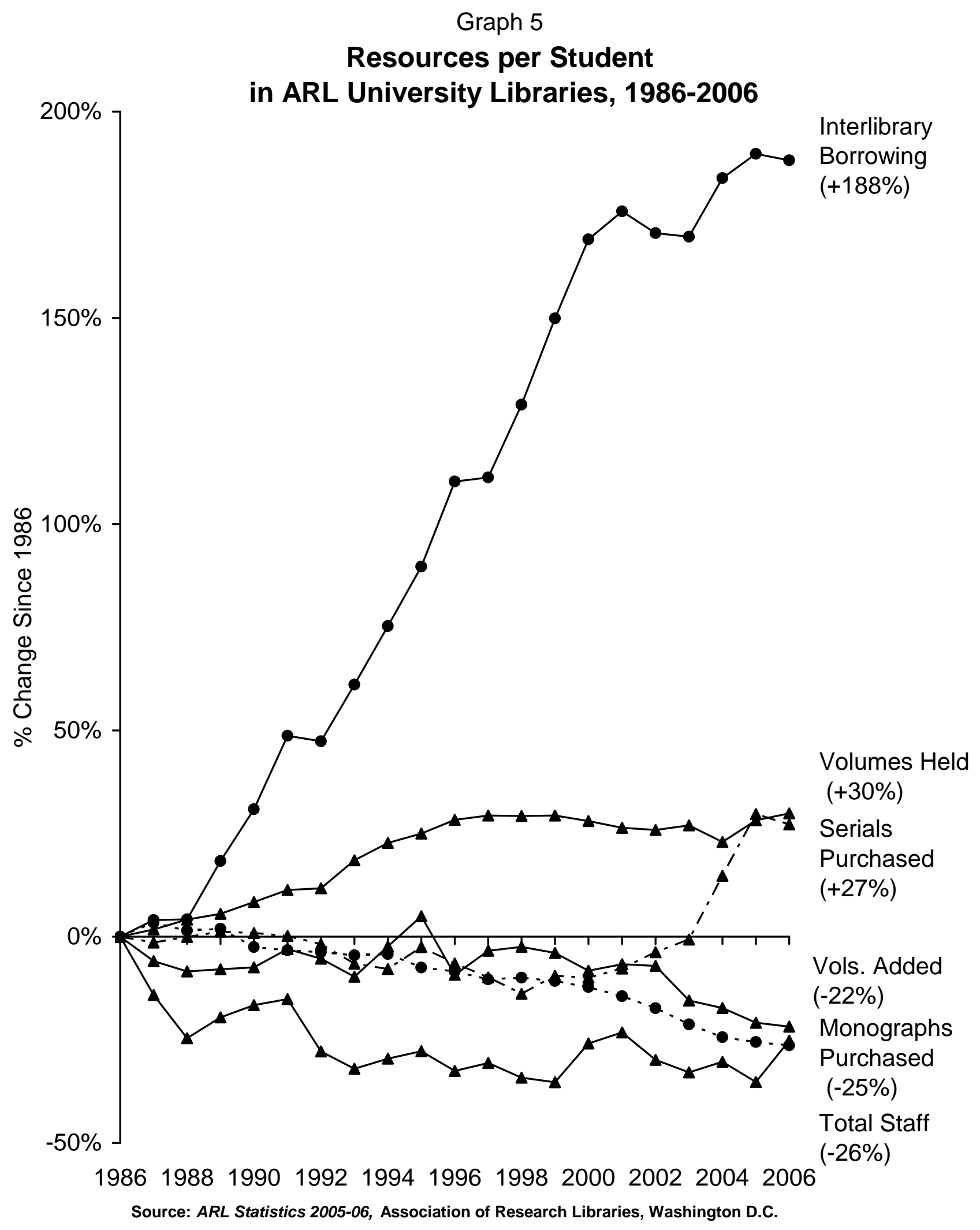

${ }^{1}$ For more information on the ARL Statistics Interactive Edition, see Martha Kyrillidou, "To Describe and Measure the 
Table 6

Electronic Materials Expenditures

In ARL University Libraries, 2005-06

\begin{tabular}{lcc}
\hline & \multicolumn{1}{c}{ Sum } & Number Reporting \\
\hline $\begin{array}{l}\text { Expenditures for Computer Files } \\
\text { (one-time/monographic purchase) }\end{array}$ & $\$ 48,793,981$ & 102 \\
$\begin{array}{l}\text { Expenditures for Electronic Serials } \\
\text { Expenditures for Bibliographic Utilities, }\end{array}$ & $\$ 26,016,318$ & 108 \\
Networks, etc. (Library) & $\$ 102,472,634$ & 102 \\
$\begin{array}{l}\text { Expenditures for Bibliographic Utilities, } \\
\text { Networks, etc. (External) }\end{array}$ & $\$ 15,946,247$ & 104 \\
Expenditures for Hardware and Software & $\$ 68,808,319$ & 107 \\
$\begin{array}{l}\text { Expenditures for Document } \\
\text { Delivery/Interlibrary Loan }\end{array}$ & $\$ 13,425,430$ & \\
\hline
\end{tabular}

Table 6 contains data on several items which previously had been collected only in the ARL Supplementary Statistics. These data are especially useful because they reflect monies spent on all electronic serials, while the ARL Statistics categories of "serials purchased" and "serials expenditures" include only those journals which provide full-text electronic versions to their subscribers. The Expenditures for Electronic Serials time series may be viewed as an alternative to the Serials Purchased series, both figures reflecting in their own way the influence the electronic serial is gaining in the modern research library.

Not only have electronic materials expenditures grown sharply in the past decade, they have grown at a rate far exceeding that of library materials expenditures overall. As shown in Graph 6, in every year of the last decade electronic materials expenditures have grown sharply, anywhere between two and ten times faster than materials expenditures have.

The average ARL university library now spends just under $41 \%$ of its materials budget on electronic materials (Tables 7A and 7B), and 23 ARL libraries report that they spent more than $50 \%$ of their materials budget on electronic materials (see Rank Order Table 20). 
Graph 6

Yearly Increases in Average:

Electronic Resources vs. Total Materials Expenditures, 1993-2006

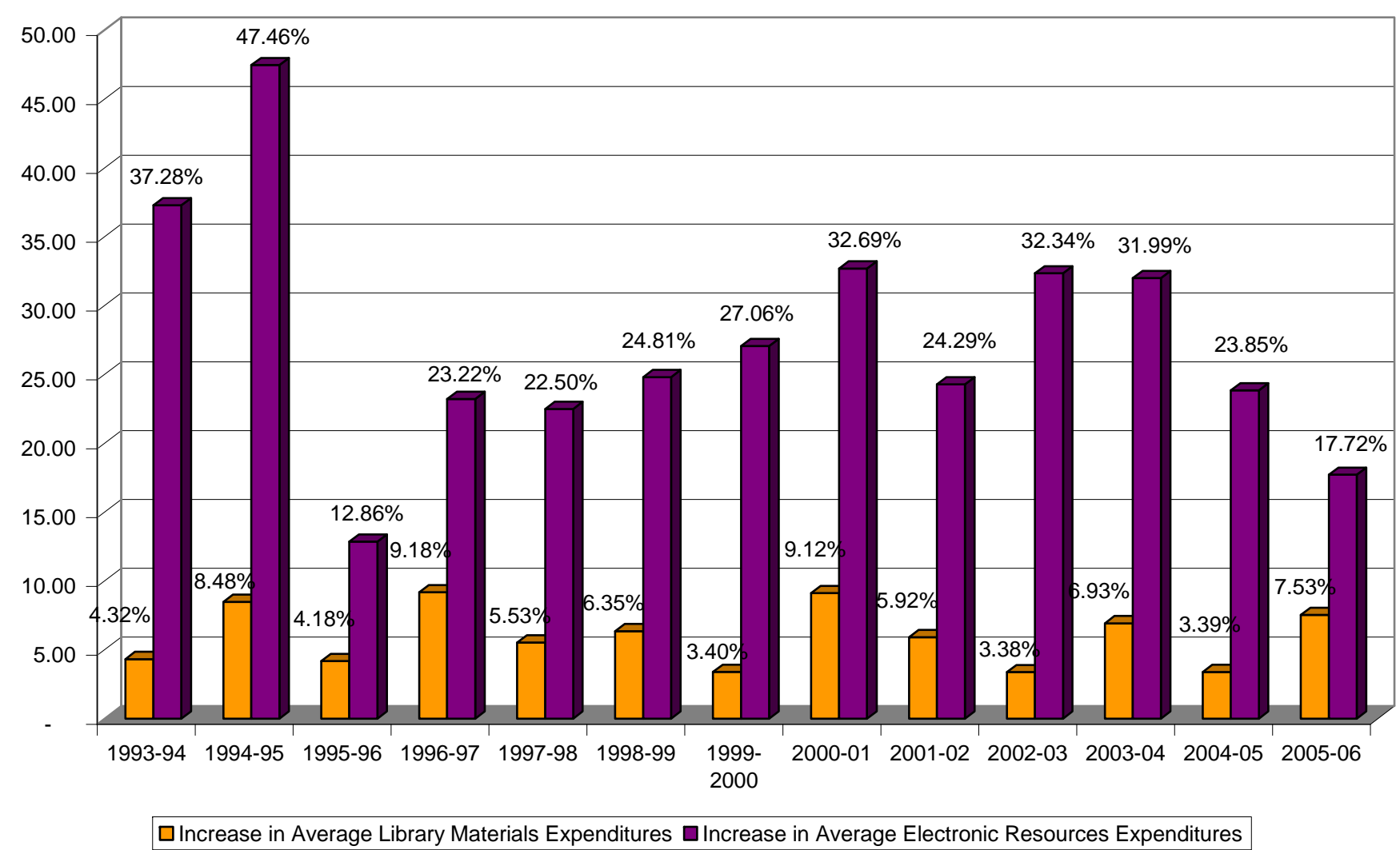


TABLE 7A

ELECTRONIC RESOURCES AND MATERIALS EXPENDITURES IN ARL UNIVERSITY LIBRARIES, 1992-2002

\begin{tabular}{|c|c|c|c|c|c|c|c|c|c|c|}
\hline & $1992-93$ & 1993-94 & 1994-95 & $1995-96$ & 1996-97 & $1997-98$ & 1998-99 & $1999-2000^{*}$ & $2000-01$ & 2001-02* \\
\hline \multicolumn{11}{|c|}{ a. Computer File Expenditures (monographic/onetime) } \\
\hline Total & $14,147,625$ & $20,132,553$ & $22,030,727$ & $24,639,822$ & $8,013,055$ & $11,189,103$ & $10,848,219$ & $14,727,984$ & $15,297,096$ & $16,748,194$ \\
\hline Average & 172,532 & 236,854 & 247,536 & 262,126 & 87,098 & 122,957 & 121,890 & 161,846 & 159,345 & 167,482 \\
\hline Median & 148,158 & 212,936 & 217,988 & 219,178 & 47,932 & 52,311 & 54,024 & 98,657 & 72,070 & 82,566 \\
\hline $\mathbf{N}$ & & 85 & 89 & 94 & 92 & 91 & 89 & 91 & 96 & 100 \\
\hline \multicolumn{11}{|c|}{ b. Electronic Serial Expenditures } \\
\hline Total & N/A & $\mathrm{N} / \mathrm{A}$ & $11,847,577$ & $15,170,971$ & $40,956,696$ & $49,497,141$ & $67,124,554$ & $84,343,868$ & $117,415,618$ & $154,418,679$ \\
\hline Average & $\mathrm{N} / \mathrm{A}$ & N/A & 188,057 & 194,500 & 401,536 & 494,971 & 639,281 & 818,873 & $1,118,244$ & $1,429,803$ \\
\hline Median & $\mathrm{N} / \mathrm{A}$ & $\mathrm{N} / \mathrm{A}$ & 156,754 & 172,805 & 355,922 & 426,722 & 571,790 & 736,317 & 992,067 & $1,272,965$ \\
\hline $\mathbf{N}$ & & & 63 & 78 & 102 & 100 & 105 & 103 & 105 & 108 \\
\hline \multicolumn{11}{|c|}{ c. Total Electronic Resources (Total a+b) } \\
\hline Total & $14,147,625$ & $20,132,553$ & $33,878,304$ & $39,810,793$ & $50,512,984$ & $60,686,244$ & $77,972,773$ & $99,071,852$ & $132,712,714$ & $171,166,873$ \\
\hline Average & 172,532 & 236,854 & 349,261 & 394,166 & 485,702 & 594,963 & 742,598 & 943,541 & $1,252,007$ & $1,556,062$ \\
\hline Median & 148,158 & 212,936 & 278,404 & 332,128 & 420,741 & 495,011 & 645,495 & 931,210 & $1,129,298$ & $1,377,874$ \\
\hline $\mathbf{N}$ & 82 & 85 & 97 & 101 & 104 & 102 & 105 & 105 & 106 & 110 \\
\hline \multicolumn{11}{|c|}{ Total Library Materials Expenditures** } \\
\hline Total & $393,271,073$ & $425,287,651$ & $526,496,347$ & $571,145,986$ & $642,123,715$ & $664,600,663$ & $727,623,160$ & $773,321,519$ & $828,778,808$ & $910,930,849$ \\
\hline \begin{tabular}{|l|} 
Average \\
\end{tabular} & $4,795,989$ & $5,003,384$ & $5,427,797$ & $5,654,911$ & $6,174,266$ & $6,515,692$ & $6,929,744$ & $7,364,967$ & $7,818,668$ & $8,281,189$ \\
\hline Median & $4,242,887$ & $4,527,122$ & $4,714,384$ & $4,975,353$ & $5,529,606$ & $5,643,070$ & $5,991,177$ & $6,545,146$ & $7,028,134$ & $7,566,727$ \\
\hline $\mathbf{N}$ & 82 & 85 & 97 & 101 & 104 & 102 & 105 & 105 & 106 & 110 \\
\hline \multicolumn{11}{|c|}{ Electronic Resources Expenditures as a Percent of Total Materials Expenditures } \\
\hline \begin{tabular}{|l} 
Average \\
\end{tabular} & 3.60 & 4.75 & 6.39 & 6.83 & 7.76 & 8.85 & 10.56 & 12.88 & 16.25 & 19.60 \\
\hline Median & N/A & 4.45 & 5.33 & 6.42 & 7.51 & 8.29 & 10.18 & 12.75 & 14.80 & 18.15 \\
\hline $\mathbf{N}$ & 82 & 85 & 97 & 101 & 104 & 102 & 105 & 105 & 106 & 110 \\
\hline \multicolumn{11}{|c|}{ Expenditures for Bibliographic Utilities, Networks, etc. (External) } \\
\hline Total & N/A & N/A & N/A & N/A & $\$ 3,827,348$ & $4,695,737$ & $7,442,962$ & $9,523,348$ & $14,655,078$ & $20,373,560$ \\
\hline Average & N/A & N/A & $\mathrm{N} / \mathrm{A}$ & $\mathrm{N} / \mathrm{A}$ & $\$ 136,691$ & 142,295 & 201,161 & 250,614 & 311,810 & 424,449 \\
\hline Median & $\mathrm{N} / \mathrm{A}$ & $\mathrm{N} / \mathrm{A}$ & $\mathrm{N} / \mathrm{A}$ & N/A & $\$ 120,096$ & 128,795 & 145,280 & 204,598 & 198,289 & 336,690 \\
\hline $\mathbf{N}$ & & & & & 28 & 33 & 37 & 38 & 47 & 48 \\
\hline
\end{tabular}

*In a recent review of past Supplementary Statistics data, some figures previously published in these columns were found to be incorrect and subsequently revised.

**Figures reflect Materials Expenditures only from those institutions that reported nonzero figures for Total Electronic Resources. 
TABLE 7B

ELECTRONIC RESOURCES AND MATERIALS EXPENDITURES IN ARL UNIVERSITY LIBRARIES, 2002-2006

\begin{tabular}{|c|c|c|c|c|}
\hline & 2002-03 & 2003-04 & 2004-05 & 2005-06 \\
\hline \multicolumn{5}{|c|}{ a. Computer File Expenditures (monographic/onetime) } \\
\hline Total & $\$ 23,275,683$ & $\$ 32,098,404$ & $\$ 38,744,076$ & $\$ 48,793,981$ \\
\hline Average & $\$ 225,978$ & $\$ 314,690$ & $\$ 372,539$ & $\$ 478,372$ \\
\hline Median & $\$ 111,266$ & $\$ 191,148$ & $\$ 210,576$ & $\$ 336,338$ \\
\hline $\mathbf{N}$ & 103 & 102 & 104 & 102 \\
\hline \multicolumn{5}{|c|}{ b. Electronic Serial Expenditures } \\
\hline Total & $\$ 205,300,292$ & $\$ 269,601,241$ & $\$ 328,166,027$ & $\$ 383,127,163$ \\
\hline Average & $\$ 1,849,552$ & $\$ 2,450,920$ & $\$ 3,038,574$ & $\$ 3,547,474$ \\
\hline Median & $\$ 1,649,361$ & $\$ 2,348,463$ & $\$ 2,824,962$ & $\$ 3,349,709$ \\
\hline $\mathbf{N}$ & 111 & 110 & 108 & 108 \\
\hline \multicolumn{5}{|c|}{ c. Total Electronic Resources (Total a+b) } \\
\hline Total & $\$ 228,575,974$ & $\$ 301,699,645$ & $\$ 366,910,103$ & $\$ 431,921,144$ \\
\hline Average & $\$ 2,059,243$ & $\$ 2,718,015$ & $\$ 3,366,147$ & $\$ 3,962,579$ \\
\hline Median & $\$ 1,775,865$ & $\$ 2,705,847$ & $\$ 3,144,841$ & $\$ 3,792,873$ \\
\hline $\mathbf{N}$ & 111 & 111 & 109 & 109 \\
\hline \multicolumn{5}{|c|}{ Total Library Materials Expenditures** } \\
\hline Total & $\$ 950,275,167$ & $\$ 1,016,121,605$ & $\$ 1,031,619,722$ & $\$ 1,109,340,878$ \\
\hline Average & $\$ 8,561,038$ & $\$ 9,154,249$ & $\$ 9,464,401$ & $\$ 10,177,439$ \\
\hline Median & $\$ 7,707,153$ & $\$ 8,276,175$ & $\$ 8,662,668$ & $\$ 9,156,974$ \\
\hline $\mathbf{N}$ & 111 & 111 & 109 & 109 \\
\hline \multicolumn{5}{|c|}{ Electronic Resources Expenditures as a Percent of Total Materials Expenditures } \\
\hline Average & 25.02 & 31.33 & 37.46 & 40.93 \\
\hline Median & 22.01 & 29.81 & 37.53 & 43.14 \\
\hline $\mathbf{N}$ & 111 & 111 & 109 & 109 \\
\hline \multicolumn{5}{|c|}{ Expenditures for Bibliographic Utilities, Networks, etc. (External) ${ }^{+}$} \\
\hline Total & $\$ 21,470,716$ & $\$ 17,420,520$ & $\$ 16,082,790^{+}$ & $\$ 15,930,476$ \\
\hline Average & $\$ 438,178$ & $\$ 335,010$ & $\$ 349,626^{+}$ & $\$ 318,610$ \\
\hline Median & $\$ 250,000$ & $\$ 94,837$ & $\$ 149,396^{+}$ & $\$ 143,649$ \\
\hline $\mathbf{N}$ & 49 & 52 & $46^{+}$ & 50 \\
\hline
\end{tabular}

** Figures reflect Materials Expenditures only from those institutions that reported nonzero figures for Total Electronic Resources. 



\section{ARL STATISTICS INTERACTIVE EDITION ON THE WORLD WIDE WEB}

The ARL Statistics are available through an interactive edition on the Web, supplementing previously available data from 1992-93. Maintained and supported by the Geospatial and Statistical Data Center at the University of Virginia, this site offers researchers access to ARL Statistics with documentation. ${ }^{1}$

The site offers maps and graphics depicting current library trends. For the particularly adventurous, the site links to an interactive graphics and statistics page, allowing users to manipulate their data queries. One may chose univariate, bivariate, or multivariate statistics in addition to a vast array of selections with which to customize a query. The interactive pages also include options for regional aggregates, customized samples of observations, simple rank order tabulations of data, and the ability to create ratios of any two variables. These and other capabilities are located at:

$$
\text { http://fisher.lib.virginia.edu/arl/ }
$$

The ARL Statistics datafiles since 1908 can be retrieved in spreadsheet format from:

$$
\text { http://www.arl.org/stats/annualsurveys/arlstats/mrstat.shtml }
$$

The ARL Statistics and Measurement Program Web site is located at:

$$
\text { http://www.arl.org/stats/ }
$$



ARL LiBRARY DATA TABLES, 2005-06 
ARL LibRARY DATA TABLES, 2005-06

COLLECTIONS

$\begin{array}{rrrrrrr}\text { Volumes } & \text { Volumes } & \text { Volumes } & \text { Monographs } & \text { Current } & \text { Current } & \text { Current } \\ \text { in Library } & \text { Added } & \text { Added } & \text { Purchased } & \text { Serials } & \text { Serials } & \text { Serials } \\ & \text { (Gross) } & \text { (Net) } & \text { (Volumes) } & \text { Purchased } & \text { Received } & \text { Total }\end{array}$

(Survey Question \#)

(1) (1.b.i) (1.b)

(2) (4.a)

(4.b)

(4) INSTITUTION

Notes

\begin{tabular}{|c|c|c|c|c|c|c|c|c|}
\hline ALABAMA & BGLM+ & $2,566,978$ & 52,804 & 48,778 & 22,388 & 25,904 & 8,557 & 34,461 \\
\hline ALBERTA & GL+ & $6,416,254$ & 255,975 & 225,858 & UA/NA & 55,708 & 13,659 & 69,367 \\
\hline ARIZONA & BGLM+ & $5,533,482$ & 87,095 & 15,918 & 49,812 & UA/NA & UA/NA & 33,899 \\
\hline ARIZONA STATE & GL+ & $4,254,719$ & 89,216 & 76,475 & 69,903 & 19,726 & 13,474 & 33,200 \\
\hline AUBURN & BG+ & $2,984,279$ & 35,713 & 28,420 & 18,338 & 10,072 & 8,622 & 18,694 \\
\hline BOSTON & GLM+ & $2,449,521$ & 63,186 & 22,268 & 18,175 & UA/NA & UA/NA & 40,757 \\
\hline BOSTON COLLEGE & $\mathrm{GL}^{+}$ & $2,407,253$ & 46,782 & 36,114 & UA/NA & 40,204 & 13,722 & 53,926 \\
\hline BRIGHAM YOUNG & BGL+ & $3,738,847$ & 120,649 & 117,270 & 93,712 & 19,858 & 11,037 & 30,895 \\
\hline BRITISH COLUMBIA & GLM+ & $5,820,527$ & 133,885 & 133,885 & UA/NA & UA/NA & UA/NA & 56,959 \\
\hline BROWN & $\mathrm{M}+$ & $3,568,944$ & 59,889 & 59,234 & 40,070 & 26,373 & 13,709 & 40,082 \\
\hline CALIFORNIA, BERKELEY & $\mathrm{L}+$ & $10,094,417$ & 186,987 & 158,821 & UA/NA & 89,249 & 25,611 & 114,860 \\
\hline CALIFORNIA, DAVIS & GLM+ & $3,549,004$ & 68,375 & 58,636 & UA/NA & UA/NA & UA/NA & 47,008 \\
\hline CALIFORNIA, IRVINE & BGM+ & $2,605,003$ & 118,034 & 110,017 & 28,015 & 35,648 & 17,402 & 53,050 \\
\hline CALIFORNIA, LOS ANGELES & $\mathrm{LM}+$ & $8,157,182$ & 106,767 & 68,547 & 65,597 & $\mathrm{UA} / \mathrm{NA}$ & $\mathrm{UA} / \mathrm{NA}$ & 77,509 \\
\hline CALIFORNIA, RIVERSIDE & BG & $2,435,296$ & 66,733 & 66,453 & 36,001 & 21,874 & 8,067 & 29,941 \\
\hline CALIFORNIA, SAN DIEGO & $\mathrm{M}+$ & $3,236,219$ & 108,376 & 89,461 & 83,135 & 20,129 & 11,959 & 32,088 \\
\hline CALIFORNIA, SANTA BARBARA & & $2,880,294$ & 43,820 & 42,542 & 27,218 & 20,237 & 16,725 & 36,962 \\
\hline CASE WESTERN RESERVE & GLM+ & $2,495,769$ & 28,365 & 24,265 & 12,639 & 11,228 & 7,194 & 18,422 \\
\hline CHICAGO & BGLM+ & $7,765,583$ & 208,122 & 120,869 & 114,686 & UA/NA & UA/NA & 43,390 \\
\hline CINCINNATI & GLM+ & $3,209,337$ & 87,840 & 83,429 & 39,729 & 40,890 & 1,375 & 42,265 \\
\hline COLORADO & BG+ & $3,641,096$ & 82,741 & 62,867 & 30,920 & 11,623 & 18,598 & 30,221 \\
\hline COLORADO STATE & $\mathrm{G}^{+}$ & $2,056,928$ & 46,823 & 30,420 & 38,589 & 20,069 & 11,313 & 31,382 \\
\hline COLUMBIA & LM+ & $9,455,312$ & 189,507 & 156,276 & 118,294 & 82,764 & 34,500 & 117,264 \\
\hline CONNECTICUT & GLM+ & $3,629,755$ & 38,920 & 32,332 & 29,929 & 41,626 & 15,644 & 57,270 \\
\hline CORNELL & GL+ & $7,785,263$ & 127,998 & 118,294 & UA/NA & 62,320 & 15,072 & 77,392 \\
\hline DARTMOUTH & GM+ & $2,690,395$ & 48,139 & 41,762 & 28,985 & 49,274 & 6,115 & 55,389 \\
\hline DELAWARE & $\mathrm{G}^{+}$ & $2,704,986$ & 40,076 & 37,744 & 29,101 & 7,519 & 5,013 & 12,532 \\
\hline DUKE & BLM+ & $5,665,241$ & 101,563 & 96,188 & 67,985 & 57,223 & 1,059 & 58,282 \\
\hline EMORY & LM+ & $3,184,754$ & 86,754 & 77,226 & 42,830 & 24,316 & 13,463 & 37,779 \\
\hline FLORIDA & LM+ & $4,178,355$ & 51,388 & 40,827 & 34,584 & 69,762 & 15,407 & 85,169 \\
\hline FLORIDA STATE & BLM+ & $2,947,702$ & 64,265 & 57,892 & 25,699 & 50,074 & 1,822 & 51,896 \\
\hline GEORGE WASHINGTON & BLM+ & $2,197,950$ & 46,613 & 30,370 & 27,426 & 20,421 & 4,732 & 25,153 \\
\hline GEORGETOWN & BGLM+ & $2,489,528$ & 74,321 & 16,320 & 52,381 & 25,937 & 5,259 & 31,196 \\
\hline GEORGIA & $\mathrm{GL}^{+}$ & $4,345,939$ & 75,195 & 71,865 & 47,805 & 26,482 & 12,744 & 39,226 \\
\hline GEORGIA TECH & $\mathrm{G}^{+}$ & $2,449,323$ & 39,428 & 38,359 & 6,327 & 12,682 & 21,894 & 34,576 \\
\hline GUELPH & $\mathrm{G}^{+}$ & $1,557,742$ & 20,131 & $-1,336$ & 16,950 & 11,304 & 500 & 11,804 \\
\hline HARVARD & GLM+ & $15,826,570$ & 292,658 & 240,336 & UA/NA & UA/NA & UA/NA & 98,988 \\
\hline HAWAII & $\mathrm{L}+$ & $3,407,167$ & 58,083 & $-75,538$ & 32,248 & 19,616 & 13,675 & 33,291 \\
\hline HOUSTON & BGL+ & $2,231,199$ & 80,780 & 70,833 & 45,522 & 18,807 & 3,386 & 22,193 \\
\hline HOWARD & BLM+ & $2,435,163$ & 34,150 & 33,703 & 4,670 & 9,823 & 1,510 & 11,333 \\
\hline ILLINOIS, CHICAGO & GM+ & $2,324,857$ & 46,373 & 40,208 & 28,866 & UA/NA & UA/NA & 50,325 \\
\hline ILLINOIS, URBANA & GL+ & $10,524,935$ & 158,914 & 153,475 & 65,445 & 62,848 & 565 & 63,413 \\
\hline
\end{tabular}

UA/NA - Unavailable or Not Applicable

L - Includes Law library

M- Includes Medical library
+ - See Footnotes

$\mathrm{B}$ - Basis of volume count is bibliographic

G - Government documents not included in serials count 


\section{ARL LibRARY DATA TABLES, 2005-06 COLLECTIONS}

\begin{tabular}{|c|c|c|c|c|c|c|c|}
\hline $\begin{array}{r}\text { Microform } \\
\text { Units }\end{array}$ & $\begin{array}{r}\text { Government } \\
\text { Documents }\end{array}$ & $\begin{array}{r}\text { Computer } \\
\text { Files }\end{array}$ & $\begin{array}{r}\text { Archives } \\
\text { and } \\
\text { Manus. }\end{array}$ & $\begin{array}{r}\text { Carto- } \\
\text { graphic } \\
\text { Materials }\end{array}$ & $\begin{array}{r}\text { Graphic } \\
\text { Materials }\end{array}$ & $\begin{array}{r}\text { Audio } \\
\text { Materials }\end{array}$ & $\begin{array}{r}\text { Video and } \\
\text { Film }\end{array}$ \\
\hline
\end{tabular}

(6)

(7)

(8)

(9)

(10)

(11)

(12)

(13)

(Survey Question \#) INSTITUTION

\begin{tabular}{|c|c|c|c|c|c|c|c|c|}
\hline $4,045,077$ & 934,159 & 6,992 & 32,794 & 307,452 & 189,324 & 22,950 & 5,480 & ALABAMA \\
\hline $3,726,833$ & 0 & 9,059 & 22,084 & $1,512,114$ & UA/NA & UA/NA & UA/NA & ALBERTA \\
\hline $6,247,714$ & 0 & 16,534 & 9,630 & 265,924 & 354,064 & 42,481 & 25,962 & ARIZONA \\
\hline $7,712,343$ & 800,678 & 6,308 & 24,354 & 230,700 & $1,218,684$ & 64,782 & 33,734 & ARIZONA STATE \\
\hline $2,690,621$ & 241,622 & 2,759 & 10,242 & 150,758 & 75,327 & 14,167 & 4,930 & AUBURN \\
\hline $4,612,586$ & UA/NA & 1,838 & 46,311 & 1,700 & $\mathrm{UA} / \mathrm{NA}$ & 55,353 & 1,402 & BOSTON \\
\hline $4,102,631$ & 220,915 & 4,640 & 7,484 & 17,779 & 127,077 & 19,687 & 20,125 & BOSTON COLLEGE \\
\hline $3,837,384$ & 418,406 & UA/NA & 39,977 & 255,854 & $1,239,477$ & 190,030 & 23,541 & BRIGHAM YOUNG \\
\hline $5,225,508$ & $\mathrm{UA} / \mathrm{NA}$ & 26,377 & 12,366 & 588,737 & 893,245 & 97,566 & 29,512 & BRITISH COLUMBIA \\
\hline $1,947,043$ & 0 & 5,505 & 10,493 & 148,778 & 950,342 & 74,784 & 7,536 & BROWN \\
\hline $6,891,881$ & 508,081 & 16,465 & 81,790 & 435,561 & $4,629,882$ & 88,922 & 44,286 & CALIFORNIA, BERKELEY \\
\hline $4,223,277$ & 74,849 & 11,293 & 17,226 & 260,965 & 235,227 & 14,709 & 3,092 & CALIFORNIA, DAVIS \\
\hline $2,889,312$ & 200,063 & 9,979 & 3,896 & 11,148 & 71,661 & 10,567 & 10,575 & CALIFORNIA, IRVINE \\
\hline $6,181,047$ & 69,577 & 14,388 & 58,639 & 632,725 & $4,143,433$ & 264,454 & 27,054 & CALIFORNIA, LOS ANGELES \\
\hline $2,182,650$ & 297,963 & 9,079 & 4,011 & 105,685 & 21,217 & 19,287 & 7,550 & CALIFORNIA, RIVERSIDE \\
\hline $3,260,769$ & 0 & 12,841 & 13,072 & 129,309 & 389,345 & 120,666 & 16,402 & CALIFORNIA, SAN DIEGO \\
\hline $3,771,751$ & 419,256 & 13,011 & 16,691 & $3,928,207$ & 1,824 & 131,046 & 9,563 & CALIFORNIA, SANTA BARBARA \\
\hline $2,565,701$ & 24,717 & 8,051 & 1,790 & 64,011 & 72,556 & 31,114 & 20,815 & CASE WESTERN RESERVE \\
\hline $3,044,663$ & $\mathrm{UA} / \mathrm{NA}$ & 10,025 & 33,341 & 430,643 & 0 & 51,059 & 4,533 & CHICAGO \\
\hline $3,346,254$ & 34,353 & 13,036 & 25,324 & 170,166 & 298,038 & 57,435 & 21,684 & CINCINNATI \\
\hline $6,843,252$ & 966,668 & 11,742 & 37,932 & 214,299 & 368,926 & 66,382 & 14,999 & COLORADO \\
\hline $1,197,144$ & 227,953 & 6,802 & 4,305 & 44,322 & 4,292 & 929 & 701 & COLORADO STATE \\
\hline $6,245,400$ & 194,156 & 45,724 & 236,640 & 138,695 & 814,224 & 95,154 & 28,523 & COLUMBIA \\
\hline $4,404,479$ & UA/NA & 3,687 & 25,616 & 232,211 & 2,677 & 58,776 & 9,701 & CONNECTICUT \\
\hline $8,327,016$ & UA/NA & 17,279 & 63,628 & 250,050 & 48,917 & 115,025 & 28,699 & CORNELL \\
\hline $2,584,144$ & 0 & 10,389 & 21,373 & 188,846 & 497,887 & 35,579 & 13,973 & DARTMOUTH \\
\hline $3,428,291$ & 448,183 & 35,155 & 4,710 & 126,541 & 812 & 1,905 & 16,557 & DELAWARE \\
\hline $4,215,023$ & $1,248,520$ & 54,084 & 50,851 & 131,971 & 900,192 & 51,484 & 68,627 & DUKE \\
\hline $6,157,328$ & 303,566 & 10,382 & 21,348 & 16,726 & 2,912 & 47,341 & 36,580 & EMORY \\
\hline $7,752,132$ & $1,551,630$ & 7,763 & 8,430 & 842,374 & 99,430 & 13,602 & 41,738 & FLORIDA \\
\hline $9,094,077$ & 855,373 & 9,771 & 5,720 & 173,231 & 1,226 & 59,465 & 17,638 & FLORIDA STATE \\
\hline $2,900,748$ & 0 & 685 & 19,238 & 18,694 & 169,611 & 10,114 & 16,317 & GEORGE WASHINGTON \\
\hline $3,945,820$ & $\mathrm{UA} / \mathrm{NA}$ & 7,098 & 17,138 & 1,809 & 374,269 & 17,630 & 20,231 & GEORGETOWN \\
\hline $6,580,560$ & $1,060,065$ & 18,287 & 56,710 & 613,212 & $1,635,071$ & 126,715 & 145,677 & GEORGIA \\
\hline $4,564,518$ & 0 & 15,282 & 5,279 & 197,659 & 107,937 & 1,866 & 5,092 & GEORGIA TECH \\
\hline $2,326,827$ & 0 & 3,095 & 12,858 & 73,893 & 7,566 & 12,223 & 8,021 & GUELPH \\
\hline $10,008,356$ & UA/NA & UA/NA & UA/NA & UA/NA & UA/NA & UA/NA & UA/NA & HARVARD \\
\hline $3,502,277$ & 0 & 5,120 & 5,933 & 15,752 & 3,095 & 33,878 & 30,570 & HAWAII \\
\hline $5,917,649$ & 680,971 & 2,144 & 2,979 & 5,651 & 526 & 4,732 & 5,888 & HOUSTON \\
\hline $3,776,923$ & 2,145 & 42 & 18,626 & 18 & 157,680 & 25,129 & 4,541 & HOWARD \\
\hline $3,948,866$ & 776,943 & UA/NA & 34,720 & 187,780 & 866,351 & 12,615 & 10,650 & ILLINOIS, CHICAGO \\
\hline $8,508,526$ & 0 & 13,399 & 35,727 & 663,581 & 90,627 & 154,141 & 23,769 & ILLINOIS, URBANA \\
\hline
\end{tabular}




\section{ARL LIBRARY DATA TABLES, 2005-06 COLLECTIONS}

$\begin{array}{rrrrrrr}\text { Volumes } & \text { Volumes } & \text { Volumes } & \text { Monographs } & \text { Current } & \text { Current } & \text { Current } \\ \text { in Library } & \text { Added } & \text { Added } & \text { Purchased } & \text { Serials } & \text { Serials } & \text { Serials } \\ & \text { (Gross) } & (\text { Net }) & \text { (Volumes) } & \text { Purchased } & \text { Received } & \text { Total }\end{array}$

(Survey Question \#) INSTITUTION
(1) (1.b.i)

Notes

\begin{tabular}{|c|c|c|c|c|c|c|c|c|}
\hline INDIANA & $\mathrm{BL}+$ & $7,374,784$ & 155,895 & 132,855 & 63,013 & 71,330 & 8,097 & 79,427 \\
\hline IOWA & GLM+ & $4,592,560$ & 107,046 & 41,343 & 59,854 & 32,909 & 18,465 & 51,374 \\
\hline IOWA STATE & $\mathrm{G}^{+}$ & $2,473,075$ & 48,314 & 28,812 & 28,581 & 18,610 & 12,646 & 31,256 \\
\hline JOHNS HOPKINS & BGM+ & $3,686,575$ & 53,306 & 44,934 & 72,596 & 48,631 & 25,613 & 74,244 \\
\hline KANSAS & LM+ & $4,194,283$ & 74,974 & 72,710 & 37,802 & 25,663 & 22,374 & 48,037 \\
\hline KENT STATE & $\mathrm{G}^{+}$ & $2,758,324$ & 46,660 & 42,338 & 11,808 & 11,941 & 1,785 & 13,726 \\
\hline KENTUCKY & GLM+ & $3,406,377$ & 83,729 & 80,483 & 35,306 & 21,132 & 10,765 & 31,897 \\
\hline LAVAL & GLM+ & $2,785,210$ & 60,462 & 53,869 & 32,437 & 23,897 & 4,520 & 28,417 \\
\hline LOUISIANA STATE & LM+ & $3,499,137$ & 57,772 & 30,901 & 21,468 & UA/NA & UA/NA & 60,889 \\
\hline LOUISVILLE & GLM+ & $2,081,825$ & 67,393 & 65,617 & 55,477 & 37,931 & 0 & 37,931 \\
\hline MCGILL & GLM+ & $3,631,326$ & 75,737 & 62,675 & 46,334 & 75,111 & 6,661 & 81,772 \\
\hline MCMASTER & GM & $2,005,280$ & 27,495 & 14,413 & 33,718 & 18,343 & 8,134 & 26,477 \\
\hline MANITOBA & GLM+ & $2,190,304$ & 40,744 & 32,156 & 47,129 & 20,494 & 8,434 & 28,928 \\
\hline MARYLAND & $\mathrm{G}^{+}$ & $3,501,054$ & 66,128 & 35,548 & 52,712 & 12,147 & 20,630 & 32,777 \\
\hline MASSACHUSETTS & $\mathrm{G}^{+}$ & $3,230,697$ & 33,462 & 26,672 & 19,301 & 33,959 & 6,790 & 40,749 \\
\hline MIT & $\mathrm{G}^{+}$ & $2,807,568$ & 44,170 & 25,162 & 22,343 & 11,826 & 10,506 & 22,332 \\
\hline MIAMI & GLM+ & $3,013,867$ & 76,138 & 61,335 & 33,996 & 50,290 & 1,059 & 51,349 \\
\hline MICHIGAN & LM+ & $8,273,050$ & 176,998 & 134,446 & UA/NA & 74,746 & 43,908 & 118,654 \\
\hline MICHIGAN STATE & BGL+ & $4,864,603$ & 80,572 & 33,742 & 36,418 & 22,767 & 17,315 & 40,082 \\
\hline MINNESOTA & BGLM+ & $6,713,629$ & 132,628 & 103,473 & 38,722 & 53,126 & 19,447 & 72,573 \\
\hline MISSOURI & BGLM+ & $3,295,378$ & 51,329 & 45,595 & 36,780 & 18,982 & 17,262 & 36,244 \\
\hline MONTREAL & GLM+ & $3,090,289$ & 64,371 & 46,012 & 52,758 & 56,723 & 5,651 & 62,374 \\
\hline NEBRASKA & GL+ & $3,113,473$ & 52,880 & 34,285 & 17,298 & 44,936 & 8,530 & 53,466 \\
\hline NEW MEXICO & $\mathrm{LM}+$ & $2,675,082$ & 47,699 & 8,112 & 23,221 & 18,143 & 6,180 & 24,323 \\
\hline NEW YORK & GLM+ & $5,144,879$ & 137,629 & 109,548 & 80,066 & 44,005 & 18,532 & 62,537 \\
\hline NORTH CAROLINA & GLM+ & $5,816,677$ & 118,374 & 105,991 & 69,290 & 30,796 & 23,795 & 54,591 \\
\hline NORTH CAROLINA STATE & $\mathrm{G}^{+}$ & $3,687,733$ & 159,765 & 156,784 & 32,571 & 22,794 & 32,049 & 54,843 \\
\hline NORTHWESTERN & GLM+ & $4,687,828$ & 102,557 & 84,004 & 43,803 & UA/NA & UA/NA & 45,259 \\
\hline NOTRE DAME & GL+ & $3,246,968$ & 76,470 & 61,042 & 54,514 & UA/NA & UA/NA & 21,622 \\
\hline $\mathrm{OHIO}$ & BGM+ & $2,722,197$ & 71,231 & 67,496 & 36,564 & 16,814 & 5,267 & 22,081 \\
\hline OHIO STATE & LM+ & $6,180,744$ & 123,424 & 63,603 & 90,377 & 21,734 & 15,079 & 36,813 \\
\hline OKLAHOMA & GLM+ & $4,918,351$ & 84,137 & 63,066 & 29,096 & 37,840 & 25,591 & 63,431 \\
\hline OKLAHOMA STATE & BGM & $2,711,255$ & 92,050 & 86,575 & 28,095 & UA/NA & UA/NA & 63,116 \\
\hline OREGON & GL+ & $2,787,476$ & 40,872 & 30,901 & 42,822 & 17,775 & 5,411 & 23,186 \\
\hline PENNSYLVANIA & LM+ & $5,880,460$ & 108,678 & 95,904 & UA/NA & UA/NA & UA/NA & 47,787 \\
\hline PENNSYLVANIA STATE & GLM+ & $5,069,854$ & 80,745 & 38,658 & 70,021 & 56,674 & 14,556 & 71,230 \\
\hline PITTSBURGH & GLM+ & $4,909,264$ & 129,443 & 122,966 & UA/NA & UA/NA & UA/NA & 50,232 \\
\hline PRINCETON & $\mathrm{B}+$ & $6,618,464$ & 130,801 & 122,867 & 81,140 & 40,498 & 1,277 & 41,775 \\
\hline PURDUE & $\mathrm{G}^{+}$ & $2,511,097$ & 58,618 & 35,855 & 23,907 & 14,154 & 22,142 & 36,296 \\
\hline QUEEN'S & BLM+ & $2,477,137$ & 45,541 & 39,969 & 25,950 & 39,918 & 5,445 & 45,363 \\
\hline RICE & $\mathrm{G}^{+}$ & $2,474,352$ & 46,741 & 37,175 & 37,175 & 11,157 & 4,697 & 15,854 \\
\hline ROCHESTER & BGM+ & $3,607,310$ & 49,221 & 42,627 & 20,591 & UA/NA & UA/NA & 26,760 \\
\hline
\end{tabular}

UA/NA - Unavailable or Not Applicable

L - Includes Law library

M- Includes Medical library
+ - See Footnotes

$\mathrm{B}$ - Basis of volume count is bibliographic

G - Government documents not included in serials count 


\section{ARL LibRARY DATA TABLES, 2005-06 COLLECTIONS}

\begin{tabular}{|c|c|c|c|c|c|c|c|}
\hline $\begin{array}{r}\text { Microform } \\
\text { Units }\end{array}$ & $\begin{array}{c}\text { Government } \\
\text { Documents }\end{array}$ & $\begin{array}{r}\text { Computer } \\
\text { Files }\end{array}$ & $\begin{array}{r}\text { Archives } \\
\text { And } \\
\text { Manus. }\end{array}$ & $\begin{array}{r}\text { Carto- } \\
\text { graphic } \\
\text { Materials }\end{array}$ & $\begin{array}{r}\text { Graphic } \\
\text { Materials }\end{array}$ & $\begin{array}{r}\text { Audio } \\
\text { Materials }\end{array}$ & $\begin{array}{r}\text { Video and } \\
\text { Film }\end{array}$ \\
\hline
\end{tabular}

(6)

(7)

(8)

(9)

(10)

(11)

(12)

(13)

(Survey Question \#) INSTITUTION

\begin{tabular}{|c|c|c|c|c|c|c|c|c|}
\hline $5,292,522$ & 314,450 & 77,039 & 36,544 & 701,100 & $3,861,908$ & 282,891 & 74,966 & INDIANA \\
\hline $7,189,464$ & 665,618 & 22,858 & 16,186 & 421,683 & 229,393 & 36,096 & 25,783 & IOWA \\
\hline $3,491,104$ & 0 & 9,084 & 16,232 & 108,440 & 872,620 & 13,981 & 51,942 & IOWA STATE \\
\hline $4,388,145$ & 80 & 6,135 & 12,713 & 216,545 & 70,418 & 28,856 & 11,320 & JOHNS HOPKINS \\
\hline $3,623,655$ & 734,016 & 12,010 & 48,708 & 337,005 & $3,070,249$ & 36,542 & 19,883 & KANSAS \\
\hline UA/NA & 0 & 56,131 & 11,122 & 7,150 & 1,253 & 14,886 & 7,543 & KENT STATE \\
\hline $6,388,872$ & $1,107,296$ & 7,626 & 26,040 & 258,827 & $2,092,925$ & 49,146 & 41,649 & KENTUCKY \\
\hline $1,330,408$ & 0 & 5,907 & 0 & 348,295 & 215,205 & 20,686 & 25,144 & LAVAL \\
\hline $6,733,022$ & 601,036 & 8,688 & 23,728 & 451,983 & UA/NA & 22,699 & 4,478 & LOUISIANA STATE \\
\hline $2,201,300$ & 0 & 0 & 20,994 & 22,260 & $1,514,744$ & 32,093 & 8,832 & LOUISVILLE \\
\hline $1,826,291$ & 672,147 & 6,949 & UA/NA & 240,711 & 258,190 & 49,197 & 37,278 & MCGILL \\
\hline $1,612,607$ & 0 & 1,524 & 12,595 & 146,720 & 10 & 30,148 & 1,316 & MCMASTER \\
\hline $1,469,948$ & 224,980 & UA/NA & 11,505 & 108,196 & 272,589 & 27,437 & 9,502 & MANITOBA \\
\hline $5,771,015$ & 188,990 & 7,430 & 38,335 & 224,851 & 344,952 & 284,373 & 38,305 & MARYLAND \\
\hline $2,580,684$ & UA/NA & 1,569 & 13,760 & 101,468 & 0 & 19,403 & 5,978 & MASSACHUSETTS \\
\hline $2,401,703$ & UA/NA & 29,656 & 22,234 & 123,842 & 452,310 & 29,626 & 4,873 & MIT \\
\hline $3,941,072$ & 0 & 5,021 & 17,485 & 53,281 & 12,708 & 52,152 & 13,942 & MIAMI \\
\hline UA/NA & UA/NA & UA/NA & UA/NA & UA/NA & UA/NA & UA/NA & UA/NA & MICHIGAN \\
\hline $6,676,334$ & 0 & 18,803 & 2,600 & 309,045 & 1,817 & 64,576 & 2,576 & MICHIGAN STATE \\
\hline $6,900,505$ & $2,637,739$ & 17,107 & 79,463 & 427,463 & UA/NA & UA/NA & UA/NA & MINNESOTA \\
\hline $7,524,227$ & $1,663,855$ & 2,133 & 10,972 & 272,221 & 618 & 24,014 & 4,107 & MISSOURI \\
\hline $1,533,723$ & 0 & 7,453 & 0 & 13,155 & 142,279 & 40,390 & 14,125 & MONTREAL \\
\hline $4,630,935$ & 552,672 & 7,283 & 9,789 & 168,290 & 103,804 & 34,534 & 2,688 & NEBRASKA \\
\hline $4,708,918$ & 655,929 & 37,709 & 15,404 & 220,535 & $1,074,924$ & 28,254 & 7,442 & NEW MEXICO \\
\hline $6,372,947$ & 479,462 & 5,579 & 32,252 & 24 & $2,336,036$ & 100,051 & 42,181 & NEW YORK \\
\hline $5,023,954$ & $1,625,932$ & 11,811 & 36,740 & 325,600 & $1,025,555$ & 289,707 & 57,920 & NORTH CAROLINA \\
\hline $5,443,193$ & 0 & 173,399 & 12,208 & 40,927 & 119,842 & 4,111 & 20,911 & NORTH CAROLINA STATE \\
\hline $4,458,193$ & 7,808 & 14,095 & 13,863 & 207,114 & 610,551 & 60,997 & 27,313 & NORTHWESTERN \\
\hline $3,871,875$ & UA/NA & 6,171 & UA/NA & 19,810 & UA/NA & 21,702 & 9,954 & NOTRE DAME \\
\hline $3,278,156$ & UA/NA & 17,885 & 12,992 & 184,908 & 97,681 & 61,251 & 39,178 & $\mathrm{OHIO}$ \\
\hline $5,912,179$ & 2,080 & 10,454 & 30,601 & 228,183 & $2,307,399$ & 62,771 & UA/NA & OHIO STATE \\
\hline $4,349,115$ & 24,065 & 5,055 & 17,496 & 155,738 & $1,732,430$ & 4,333 & 8,472 & OKLAHOMA \\
\hline $4,502,389$ & 158,729 & 14,735 & 10,172 & 300,013 & 198,349 & 9,896 & 16,366 & OKLAHOMA STATE \\
\hline $2,920,159$ & 467,921 & 2,379 & 73,377 & 758,042 & $1,318,524$ & 79,636 & 29,582 & OREGON \\
\hline $4,085,467$ & UA/NA & 9,294 & 14,254 & 136,187 & 747,302 & 67,434 & 15,194 & PENNSYLVANIA \\
\hline $5,294,473$ & 0 & 10,432 & 29,339 & 469,787 & 535,447 & 67,548 & 176,883 & PENNSYLVANIA STATE \\
\hline $7,543,846$ & UA/NA & 43,213 & 30,205 & 123,434 & 736,275 & 102,979 & 68,432 & PITTSBURGH \\
\hline $6,521,389$ & 120,460 & 10,812 & 33,481 & 330,689 & 245,577 & 89,441 & 5,845 & PRINCETON \\
\hline $3,099,407$ & 489,215 & 10,770 & 3,300 & 344,312 & 1,214 & 993 & 11,713 & PURDUE \\
\hline $3,885,140$ & $1,106,135$ & UA/NA & UA/NA & 161,868 & 28,165 & UA/NA & UA/NA & QUEEN'S \\
\hline $3,204,245$ & 55,896 & 14,749 & 6,658 & 26,997 & 33,573 & 42,979 & 11,470 & RICE \\
\hline $5,103,919$ & 443,509 & 6,821 & 14,739 & 105,600 & 7,287 & 91,533 & 15,067 & ROCHESTER \\
\hline
\end{tabular}




\section{ARL LibRARY DATA TABLES, 2005-06 COLLECTIONS}

$\begin{array}{rrrrrrr}\text { Volumes } & \text { Volumes } & \text { Volumes } & \text { Monographs } & \text { Current } & \text { Current } & \text { Current } \\ \text { in Library } & \text { Added } & \text { Added } & \text { Purchased } & \text { Serials } & \text { Serials } & \text { Serials } \\ & \text { (Gross) } & \text { (Net) } & \text { (Volumes) } & \text { Purchased } & \text { Received } & \text { Total }\end{array}$

\begin{tabular}{|c|c|c|c|c|c|c|c|c|}
\hline $\begin{array}{l}\text { (Survey Question \#) } \\
\text { INSTITUTION }\end{array}$ & Notes & (1) & (1.b.i) & (1.b) & (2) & (4.a) & (4.b) & (4) \\
\hline RUTGERS & GL+ & $4,203,237$ & 72,957 & 38,334 & 24,862 & 50,003 & 3,299 & 53,302 \\
\hline SASKATCHEWAN & GLM+ & $1,996,901$ & 35,466 & 20,295 & 21,236 & 25,996 & 11,212 & 37,208 \\
\hline SOUTH CAROLINA & BLM & $3,532,538$ & 56,298 & 54,282 & 47,881 & 15,713 & 43,142 & 58,855 \\
\hline SOUTHERN CALIFORNIA & GLM+ & $3,968,814$ & 53,232 & 47,110 & 32,191 & 56,739 & 3,979 & 60,718 \\
\hline SOUTHERN ILLINOIS & LM+ & $3,026,125$ & 36,947 & 26,389 & 41,740 & 22,204 & 18,286 & 40,490 \\
\hline SUNY-ALBANY & $\mathrm{G}^{+}$ & $2,129,103$ & 35,590 & 35,055 & 16,399 & 17,524 & 6,383 & 23,907 \\
\hline SUNY-BUFFALO & GLM+ & $3,423,148$ & 41,442 & 32,565 & 15,316 & 23,800 & 13,488 & 37,288 \\
\hline SUNY-STONY BROOK & $\mathrm{BM}+$ & $2,237,823$ & 26,287 & 22,234 & 12,035 & 27,895 & 9,602 & 37,497 \\
\hline SYRACUSE & GL+ & $3,180,427$ & 32,087 & 18,898 & 21,128 & 18,793 & 4,072 & 22,865 \\
\hline TEMPLE & BLM+ & $3,071,624$ & 55,830 & 55,160 & 51,352 & 21,614 & 7,994 & 29,608 \\
\hline TENNESSEE & GLM+ & $3,183,529$ & 57,953 & 43,023 & 12,672 & 25,567 & 7,617 & 33,184 \\
\hline TEXAS & $\mathrm{L}+$ & $9,022,363$ & 127,845 & 85,361 & 68,059 & UA/NA & UA/NA & 46,880 \\
\hline TEXAS A\&M & $\mathrm{G}^{+}$ & $3,581,226$ & 149,398 & 143,850 & 86,572 & 34,567 & 11,239 & 45,806 \\
\hline TEXAS TECH & BLM+ & $2,525,957$ & 63,179 & 60,161 & 39,280 & 37,711 & 22,637 & 60,348 \\
\hline TORONTO & GL+ & $10,536,868$ & 216,531 & 194,294 & 137,067 & 58,572 & 10,218 & 68,790 \\
\hline TULANE & LM+ & $3,076,954$ & 35,843 & $-409,869$ & 25,590 & UA/NA & UA/NA & 19,716 \\
\hline UTAH & BGLM+ & $3,254,709$ & 76,048 & 23,855 & UA/NA & UA/NA & UA/NA & 40,607 \\
\hline VANDERBILT & GLM+ & $3,264,231$ & 55,283 & 49,068 & 31,683 & 26,999 & 9,185 & 36,184 \\
\hline VIRGINIA & LM+ & $5,102,954$ & 76,325 & 49,792 & 38,954 & 24,386 & 47,446 & 71,832 \\
\hline VIRGINIA TECH & $\mathrm{G}^{+}$ & $2,268,619$ & 35,702 & 31,513 & 26,196 & 33,874 & 3,114 & 36,988 \\
\hline WASHINGTON & $\mathrm{LM}+$ & $7,111,065$ & 120,950 & 99,232 & 57,155 & UA/NA & UA/NA & 62,429 \\
\hline WASHINGTON STATE & $\mathrm{G}^{+}$ & $2,277,967$ & 29,752 & 21,738 & 17,096 & UA/NA & UA/NA & 30,122 \\
\hline WASHINGTON U.-ST. LOUIS & GLM+ & $3,947,725$ & 60,029 & 55,923 & 23,570 & 28,490 & 12,849 & 41,339 \\
\hline WATERLOO & $\mathrm{G}^{+}$ & $2,008,232$ & 23,056 & 1,345 & 21,567 & UA/NA & UA/NA & 20,524 \\
\hline WAYNE STATE & GLM+ & $3,443,247$ & 36,636 & 23,837 & 17,719 & UA/NA & UA/NA & 23,693 \\
\hline WESTERN ONTARIO & GL+ & $3,388,376$ & 52,337 & 49,141 & 26,961 & 44,122 & 2,729 & 46,851 \\
\hline WISCONSIN & GLM+ & $8,015,081$ & 121,148 & 103,247 & 49,761 & 52,339 & 16,221 & 68,560 \\
\hline YALE & GLM+ & $12,368,757$ & 249,613 & 245,720 & 160,397 & 66,597 & 7,356 & 73,953 \\
\hline YORK & $\mathrm{G}^{+}$ & $2,526,324$ & 46,039 & 37,131 & 33,158 & UA/NA & UA/NA & 9,084 \\
\hline BOSTON PUBLIC & $\mathrm{G}^{+}$ & $7,426,254$ & 136,276 & $-17,505$ & 136,276 & 8,040 & 0 & 8,040 \\
\hline CISTI & $\mathrm{G}^{+}$ & $8,149,523$ & 29,486 & 8,046 & 2,609 & 8,920 & 0 & 8,920 \\
\hline CTR FOR RESEARCH LIBS & + & $2,210,357$ & 9,790 & 9,790 & 5,481 & UA/NA & UA/NA & UA/NA \\
\hline LIBRARY OF CONGRESS & BG+ & $27,120,388$ & 445,545 & 436,441 & 153,401 & 47,109 & 352,891 & 400,000 \\
\hline NATL AGRICULTURAL LIB & $\mathrm{G}^{+}$ & $2,406,191$ & 10,577 & 9,719 & 1,886 & 5,305 & 12,506 & 17,811 \\
\hline LIB \& ARCHIVES CANADA & + & $9,511,290$ & 458,588 & 458,588 & 4,521 & 4,576 & 0 & 4,576 \\
\hline NATL LIB OF MEDICINE & $\mathrm{G}^{+}$ & $2,520,347$ & 32,923 & 8,233 & 15,167 & 19,232 & 4,659 & 23,891 \\
\hline NEW YORK PUBLIC & G & $8,983,907$ & 155,686 & 155,686 & 93,281 & 53,625 & 23,243 & 76,868 \\
\hline NEW YORK STATE & + & $2,662,974$ & 30,704 & 30,669 & 11,095 & 7,525 & 6,627 & 14,152 \\
\hline SMITHSONIAN & + & $1,571,144$ & 24,589 & 19,821 & 4,712 & 3,071 & 2,463 & 5,534 \\
\hline
\end{tabular}

UA/NA - Unavailable or Not Applicable

L - Includes Law library

M- Includes Medical library
+ - See Footnotes

B - Basis of volume count is bibliographic

G - Government documents not included in serials count 


\section{ARL LibRARY DATA TABLES, 2005-06 COLLECTIONS}

\begin{tabular}{|c|c|c|c|c|c|c|c|}
\hline $\begin{array}{r}\text { Microform } \\
\text { Units }\end{array}$ & $\begin{array}{r}\text { Government } \\
\text { Documents }\end{array}$ & $\begin{array}{r}\text { Computer } \\
\text { Files }\end{array}$ & $\begin{array}{r}\text { Archives } \\
\text { and } \\
\text { Manus. }\end{array}$ & $\begin{array}{r}\text { Carto- } \\
\text { graphic } \\
\text { Materials }\end{array}$ & $\begin{array}{r}\text { Graphic } \\
\text { Materials }\end{array}$ & $\begin{array}{r}\text { Audio } \\
\text { Materials }\end{array}$ & $\begin{array}{r}\text { Video and } \\
\text { Film }\end{array}$ \\
\hline
\end{tabular}
(6)
(7)
(8)
(9)
(10)
(11)
(12)
(13)
(Survey Question \#) INSTITUTION

\begin{tabular}{|c|c|c|c|c|c|c|c|c|}
\hline $5,935,929$ & $2,663,310$ & 14,521 & 26,767 & $\mathrm{UA} / \mathrm{NA}$ & $\mathrm{UA} / \mathrm{NA}$ & UA/NA & $\mathrm{UA} / \mathrm{NA}$ & RUTGERS \\
\hline $3,084,671$ & 414,542 & 5,183 & 268 & 73,256 & 19,386 & 17,539 & 2,098 & SASKATCHEWAN \\
\hline $5,262,414$ & 923,489 & 3,617 & 9,058 & 321,334 & 250,071 & 37,522 & 16,591 & SOUTH CAROLINA \\
\hline $6,221,738$ & 268,670 & 11,013 & 46,751 & 84,492 & $3,192,375$ & 28,695 & 31,129 & SOUTHERN CALIFORNIA \\
\hline $4,588,909$ & 313,173 & 10,357 & 26,295 & 343,873 & 16,998 & 28,389 & 15,061 & SOUTHERN ILLINOIS \\
\hline $2,876,879$ & 0 & 2,263 & 10,673 & 25,206 & 55,848 & 8,083 & 7,853 & SUNY-ALBANY \\
\hline $5,442,977$ & UA/NA & 9,175 & 12,448 & 197,650 & 132,773 & 52,352 & 9,680 & SUNY-BUFFALO \\
\hline $3,834,398$ & 339,423 & 472 & 3,168 & 132,869 & 9,457 & 40,625 & 7,827 & SUNY-STONY BROOK \\
\hline $7,401,625$ & 306,768 & 2,408 & 29,147 & 277,167 & 417,750 & 414,028 & 20,906 & SYRACUSE \\
\hline $3,214,480$ & 410 & 3,631 & 35,850 & 121,228 & $10,035,210$ & 23,839 & 6,307 & TEMPLE \\
\hline $4,225,398$ & 5,297 & 2,242 & 13,542 & 390,115 & 122,138 & 28,728 & 15,551 & TENNESSEE \\
\hline $6,570,665$ & 0 & 8,390 & 121,579 & 323,417 & $11,707,062$ & 206,499 & 51,110 & TEXAS \\
\hline $5,543,084$ & 65,519 & 11,445 & 22,743 & 232,482 & 541,136 & 17,484 & 27,928 & TEXAS A\&M \\
\hline $2,630,207$ & 4,222 & 388,931 & 30,666 & 159,063 & $1,560,156$ & 17,781 & 27,514 & TEXAS TECH \\
\hline $5,425,323$ & UA/NA & 494,135 & 38,966 & 280,271 & 762,105 & 199,736 & 29,949 & TORONTO \\
\hline $1,265,125$ & 145,911 & 1,271 & 4,712 & 13 & 559 & 2,072 & 1,555 & TULANE \\
\hline $3,619,914$ & 885,109 & 7,822 & 33,372 & 252,204 & $1,485,735$ & 47,851 & 32,062 & UTAH \\
\hline $3,043,451$ & 257,762 & 10,718 & 15,933 & 108,333 & 221,680 & 36,352 & 9,910 & VANDERBILT \\
\hline $5,474,181$ & 676,242 & 10,982 & 27,446 & 150,936 & 466,787 & 67,150 & 31,792 & VIRGINIA \\
\hline $6,318,427$ & 399,525 & 10,758 & 18,676 & 137,077 & 90,474 & 10,331 & 15,922 & VIRGINIA TECH \\
\hline $7,680,082$ & 0 & 16,969 & 69,082 & 366,288 & $1,264,662$ & 77,235 & 41,258 & WASHINGTON \\
\hline $4,229,240$ & 0 & 506 & 19,031 & 7,267 & 369,419 & 14,084 & 35,967 & WASHINGTON STATE \\
\hline $3,392,968$ & 0 & 9,433 & 14,611 & 119,693 & 167,764 & 41,117 & 56,953 & WASHINGTON U.-ST. LOUIS \\
\hline $1,708,666$ & 0 & 5,772 & 5,364 & 147,181 & 41 & 1,034 & 190 & WATERLOO \\
\hline $3,866,924$ & 388,182 & 5,611 & 74,000 & 54,923 & $2,159,039$ & 46,142 & 29,151 & WAYNE STATE \\
\hline $3,991,614$ & 0 & UA/NA & 11,844 & 15,103 & $1,600,000$ & 47,461 & 252 & WESTERN ONTARIO \\
\hline $6,277,775$ & 811,086 & 11,900 & 156,511 & 575,225 & $6,252,297$ & 345,979 & 21,398 & WISCONSIN \\
\hline $10,123,626$ & 0 & UA/NA & 82,504 & 461,040 & $2,311,733$ & 234,143 & 22,425 & YALE \\
\hline $4,057,687$ & UA/NA & 2,889 & 11,418 & 113,319 & 8,647 & 34,063 & 23,314 & YORK \\
\hline $6,769,000$ & $1,776,875$ & 126 & UA/NA & UA/NA & UA/NA & 61,137 & 94,779 & BOSTON PUBLIC \\
\hline $5,000,000$ & $\mathrm{UA} / \mathrm{NA}$ & 31,616 & 0 & $\mathrm{UA} / \mathrm{NA}$ & $\mathrm{UA} / \mathrm{NA}$ & UA/NA & 748 & CISTI \\
\hline $1,843,007$ & UA/NA & 962 & UA/NA & $\mathrm{UA} / \mathrm{NA}$ & UA/NA & UA/NA & $\mathrm{UA} / \mathrm{NA}$ & CTR FOR RESEARCH LIBS \\
\hline $14,299,103$ & 0 & 129,939 & 59,365 & $5,275,222$ & $14,370,815$ & $2,834,692$ & $1,023,909$ & LIBRARY OF CONGRESS \\
\hline $1,095,271$ & 0 & 66 & 19,270 & 66 & 3 & 0 & 153 & NATL AGRICULTURAL LIB \\
\hline UA/NA & $\mathrm{UA} / \mathrm{NA}$ & UA/NA & UA/NA & $\mathrm{UA} / \mathrm{NA}$ & $\mathrm{UA} / \mathrm{NA}$ & UA/NA & $\mathrm{UA} / \mathrm{NA}$ & LIB \& ARCHIVES CANADA \\
\hline 603,956 & 0 & 2,549 & 3,377 & 0 & 68,994 & UA/NA & 80,038 & NATL LIB OF MEDICINE \\
\hline $6,820,107$ & 0 & UA/NA & 65,117 & 433,181 & $4,380,466$ & 574,173 & 64,600 & NEW YORK PUBLIC \\
\hline $8,242,776$ & $\mathrm{UA} / \mathrm{NA}$ & 8,149 & 27,929 & 208,955 & 70,334 & 55 & 87 & NEW YORK STATE \\
\hline 190,245 & UA/NA & 1,683 & 2,124 & 139 & 2,021 & 248 & 331 & SMITHSONIAN \\
\hline
\end{tabular}

UA/NA - Unavailable or Not Applicable 
ARL LiBRARY DATA TABLES, 2005-06

SUMMARY DATA: COLleCTIONS

\begin{tabular}{|c|c|c|c|c|c|c|c|}
\hline (Survey Question \#) & $\begin{array}{r}\text { Volumes } \\
\text { in Library } \\
\\
(1) \\
\end{array}$ & $\begin{array}{r}\text { Volumes } \\
\text { Added } \\
\text { (Gross) } \\
\\
(1 . b . i)\end{array}$ & $\begin{array}{r}\text { Volumes } \\
\text { Added } \\
(\text { Net }) \\
(1 . b)\end{array}$ & $\begin{array}{r}\text { Monographs } \\
\text { Purchased } \\
\text { (Volumes) } \\
(2)\end{array}$ & $\begin{array}{r}\text { Current } \\
\text { Serials } \\
\text { Purchased } \\
\\
(4 . a) \\
\end{array}$ & $\begin{array}{r}\text { Current } \\
\text { Serials } \\
\text { Received } \\
\\
(4 . b)\end{array}$ & $\begin{array}{r}\text { Current } \\
\text { Serials } \\
\text { Total } \\
\\
(4) \\
\end{array}$ \\
\hline \multicolumn{8}{|l|}{$\begin{array}{l}\text { UNIVERSITY } \\
\text { LIBRARIES: }\end{array}$} \\
\hline Median & $3,295,378$ & 64,371 & 49,068 & 34,945 & 25,967 & 10,636 & 40,607 \\
\hline High & $15,826,570$ & 292,658 & 245,720 & 160,397 & 89,249 & 47,446 & 118,654 \\
\hline Low & $1,557,742$ & 20,131 & $-409,869$ & 4,670 & 7,519 & 0 & 9,084 \\
\hline Totals & $470,282,788$ & $9,235,435$ & $6,838,886$ & $4,296,625$ & $2,992,891$ & $1,104,065$ & $5,114,511$ \\
\hline $\begin{array}{l}\text { Number of } \\
\text { Libraries Responding }\end{array}$ & 113 & 113 & 113 & 102 & 90 & 90 & 113 \\
\hline \multicolumn{8}{|l|}{$\begin{array}{l}\text { NONUNIVERSITY } \\
\text { LIBRARIES: }\end{array}$} \\
\hline Median & $5,044,614$ & 31,814 & 14,806 & 8,288 & 8,040 & 4,659 & 14,152 \\
\hline Totals & $72,562,375$ & $1,334,164$ & $1,119,488$ & 428,429 & 157,403 & 402,389 & 559,792 \\
\hline $\begin{array}{l}\text { Number of } \\
\text { Libraries Responding }\end{array}$ & 10 & 10 & 10 & 10 & 9 & 9 & 9 \\
\hline GRAND TOTALS & $542,845,163$ & $10,569,599$ & $7,958,374$ & $4,725,054$ & $3,150,294$ & $1,506,454$ & $5,674,303$ \\
\hline
\end{tabular}




\section{ARL LiBRARY DATA TABLES, 2005-06 SUMMARY DATA: COLlECTIONS}

\begin{tabular}{|c|c|c|c|c|c|c|c|c|}
\hline $\begin{array}{r}\text { Microform } \\
\text { Units } \\
(6)\end{array}$ & $\begin{array}{r}\text { Government } \\
\text { Documents } \\
\text { (7) }\end{array}$ & $\begin{array}{r}\text { Computer } \\
\text { Files } \\
\text { (8) }\end{array}$ & $\begin{array}{r}\text { Archives } \\
\text { and } \\
\text { Manus. } \\
\text { (9) }\end{array}$ & $\begin{array}{r}\text { Carto- } \\
\text { graphic } \\
\text { Materials } \\
(10)\end{array}$ & $\begin{array}{r}\text { Graphic } \\
\text { Materials } \\
(11)\end{array}$ & $\begin{array}{r}\text { Audio } \\
\text { Materials } \\
\text { (12) }\end{array}$ & $\begin{array}{r}\text { Video and } \\
\text { Film } \\
\text { (13) }\end{array}$ & (Survey Question \#) \\
\hline & & & & & & & & $\begin{array}{l}\text { UNIVERSITY } \\
\text { LIBRARIES: }\end{array}$ \\
\hline $10,123,626$ & $2,663,310$ & 494,135 & 236,640 & $3,928,207$ & $11,707,062$ & 414,028 & 176,883 & High \\
\hline $1,197,144$ & 0 & 0 & 0 & 13 & 0 & 929 & 190 & Low \\
\hline $513,129,278$ & $36,429,472$ & $2,254,293$ & $2,951,787$ & $28,458,855$ & $92,960,376$ & $6,821,400$ & $2,428,074$ & Totals \\
\hline & & & & & & & & $\begin{array}{l}\text { NONUNIVERSITY } \\
\text { LIBRARIES: }\end{array}$ \\
\hline $5,000,000$ & 0 & 2,116 & 19,270 & 104,547 & 69,664 & 30,693 & 32,674 & Median \\
\hline $44,863,465$ & $1,776,875$ & 175,090 & 177,182 & $5,917,563$ & $18,892,633$ & $3,470,305$ & $1,264,645$ & Totals \\
\hline 9 & 5 & 8 & 7 & 6 & 6 & 6 & 8 & $\begin{array}{r}\text { Number of } \\
\text { Libraries Responding }\end{array}$ \\
\hline
\end{tabular}




\section{ARL LIBRARY DATA TABLES, 2005-06}

\section{EXPENDITURES}

\begin{tabular}{|c|c|c|c|c|c|c|c|}
\hline $\begin{array}{l}\text { (Survey Question \#) } \\
\text { INSTITUTION }\end{array}$ & Notes & Monographs & $\begin{array}{r}\text { Current } \\
\text { Serials } \\
\\
(15 . b)\end{array}$ & $\begin{array}{r}\text { Other } \\
\text { Library } \\
\text { Materials } \\
\\
(15 . c)\end{array}$ & $\begin{array}{r}\text { Misc. } \\
\text { Materials } \\
(15 . \mathrm{d})\end{array}$ & $\begin{array}{r}\text { Total } \\
\text { Library } \\
\text { Materials } \\
\\
\text { (15) }\end{array}$ & $\begin{array}{c}\text { Contract } \\
\text { Binding }\end{array}$ \\
\hline ALABAMA & BGLM+ & $1,364,194$ & $5,339,198$ & 129,093 & 38,172 & $6,870,657$ & 79,345 \\
\hline ALBERTA & GL+ & $5,460,909$ & $8,492,244$ & 0 & 637,331 & $14,590,484$ & 125,320 \\
\hline ARIZONA & BGLM+ & $2,436,915$ & $8,015,246$ & 610,666 & 599,373 & $11,662,200$ & 98,160 \\
\hline ARIZONA STATE & GL+ & $3,037,222$ & $7,437,248$ & 829,792 & 170,180 & $11,474,442$ & 154,614 \\
\hline AUBURN & BG+ & 826,193 & $4,647,678$ & 26,070 & 43,785 & $5,543,726$ & 63,026 \\
\hline BOSTON & GLM+ + & 880,149 & $5,443,573$ & $2,262,956$ & 120,936 & $8,707,614$ & 139,554 \\
\hline BOSTON COLLEGE & GL+ & $1,561,217$ & $6,143,117$ & 247,216 & 57,972 & $8,009,522$ & 138,381 \\
\hline BRIGHAM YOUNG & BGL+ & $2,104,192$ & $7,000,979$ & 58,770 & 107,835 & $9,271,776$ & 234,591 \\
\hline BRITISH COLUMBIA & GLM+ & $2,141,242$ & $9,899,713$ & 704,956 & 777,923 & $13,523,834$ & 171,459 \\
\hline BROWN & $\mathrm{M}+$ & $2,085,268$ & $5,343,805$ & 530,516 & 325,805 & $8,285,394$ & 214,064 \\
\hline CALIFORNIA, BERKELEY & $\mathrm{L}+^{+}$ & $8,428,661$ & $8,719,614$ & 304,905 & 0 & $17,453,180$ & 743,662 \\
\hline CALIFORNIA, DAVIS & GLM+ & $2,721,150$ & $4,569,504$ & $1,073,473$ & 294,942 & $8,659,069$ & 163,872 \\
\hline CALIFORNIA, IRVINE & BGM+ & $1,882,952$ & $5,392,865$ & 538,909 & UA/NA & $7,814,726$ & 69,258 \\
\hline CALIFORNIA, LOS ANGELES & $\mathrm{LM}+$ & $6,831,633$ & $7,790,034$ & 71,644 & 464,838 & $15,158,149$ & 475,508 \\
\hline CALIFORNIA, RIVERSIDE & BG & $1,332,453$ & $4,467,954$ & 119,199 & 190,266 & $6,109,872$ & 246,159 \\
\hline CALIFORNIA, SAN DIEGO & $\mathrm{M}+$ & $2,321,302$ & $5,380,965$ & 220,650 & 0 & $7,922,917$ & 275,478 \\
\hline CALIFORNIA, SANTA BARBARA & & $1,447,955$ & $3,788,375$ & 0 & 0 & $5,236,330$ & 160,231 \\
\hline CASE WESTERN RESERVE & GLM+ & 655,218 & $5,226,735$ & 97,503 & 324,683 & $6,304,139$ & 113,735 \\
\hline CHICAGO & BGLM+ & $5,112,130$ & $11,056,788$ & 293,461 & 0 & $16,462,379$ & 351,091 \\
\hline CINCINNATI & GLM+ & $2,852,173$ & $6,562,896$ & 589,013 & 204,984 & $10,209,066$ & 127,684 \\
\hline COLORADO & BG+ & $1,680,710$ & $6,289,768$ & 745,316 & 600,069 & $9,315,863$ & 66,553 \\
\hline COLORADO STATE & $\mathrm{G}^{+}$ & $1,473,739$ & $4,131,559$ & 312,585 & 347,388 & $6,265,271$ & 79,097 \\
\hline COLUMBIA & LM+ & $6,006,719$ & $12,831,465$ & $1,291,297$ & 888,310 & $21,017,791$ & 640,062 \\
\hline CONNECTICUT & GLM+ & $1,043,080$ & $6,369,593$ & 126,948 & 315,258 & $7,854,879$ & 241,033 \\
\hline CORNELL & GL+ & $5,637,126$ & $7,340,695$ & $1,165,044$ & 238,714 & $14,381,579$ & 173,302 \\
\hline DARTMOUTH & $\mathrm{GM}+$ & $1,982,243$ & $5,669,207$ & 97,023 & 0 & $7,748,473$ & 114,383 \\
\hline DELAWARE & $\mathrm{G}^{+}$ & $2,147,028$ & $5,283,118$ & 440,259 & 135,281 & $8,005,686$ & 85,205 \\
\hline DUKE & BLM+ & $3,663,728$ & $9,201,728$ & 108,716 & 986,830 & $13,961,002$ & 153,551 \\
\hline EMORY & LM+ & $4,698,327$ & $5,903,787$ & $3,256,737$ & 224,758 & $14,083,609$ & 131,450 \\
\hline FLORIDA & $\mathrm{LM}+$ & $1,813,615$ & $8,347,943$ & 184,143 & 475,387 & $10,821,088$ & 177,281 \\
\hline FLORIDA STATE & BLM+ & $1,349,343$ & $4,804,159$ & $1,291,820$ & 204,732 & $7,650,054$ & 70,475 \\
\hline GEORGE WASHINGTON & BLM+ & $2,795,073$ & $5,633,307$ & 26,302 & $1,002,529$ & $9,457,211$ & 133,555 \\
\hline GEORGETOWN & BGLM+ & $2,680,496$ & $6,863,022$ & 878,507 & 361,608 & $10,783,633$ & 107,276 \\
\hline GEORGIA & GL+ & $2,456,505$ & $7,620,806$ & 698,069 & 442,431 & $11,217,811$ & 262,447 \\
\hline GEORGIA TECH & $\mathrm{G}^{+}$ & 448,097 & $4,105,463$ & 753,287 & 0 & $5,306,847$ & 37,423 \\
\hline GUELPH & $\mathrm{G}^{+}$ & $1,044,375$ & $3,245,327$ & 318,064 & 431,290 & $5,039,056$ & 27,649 \\
\hline HARVARD & GLM+ & $9,816,824$ & $9,911,522$ & $8,365,853$ & 44,728 & $28,138,927$ & $1,017,350$ \\
\hline HAWAII & $\mathrm{L}^{+}$ & $1,962,814$ & $5,648,264$ & 0 & 0 & $7,611,078$ & 158,318 \\
\hline HOUSTON & BGL+ & $1,642,148$ & $5,684,365$ & 448,191 & 342,489 & $8,117,193$ & 95,491 \\
\hline HOWARD & BLM+ + & 198,314 & $2,923,720$ & 289,622 & 0 & $3,411,656$ & 59,192 \\
\hline ILLINOIS, CHICAGO & GM+ & $1,502,978$ & $6,353,550$ & 310,095 & 0 & $8,166,623$ & 65,712 \\
\hline ILLINOIS, URBANA & GL+ & $2,444,839$ & $8,634,385$ & 483,488 & 480,960 & $12,043,672$ & 267,856 \\
\hline
\end{tabular}

UA/NA - Unavailable or Not Applicable

L - Includes Law library

M- Includes Medical library
+ - See Footnotes

$\mathrm{B}$ - Basis of volume count is bibliographic

G - Government documents not included in serials count 


\section{ARL LibRARY DATA TABLES, 2005-06 EXPENDITURES}

$\begin{array}{rrrrrr}\text { Salaries } & \text { Salaries } & \text { Salaries } & \text { Total } & \text { Other } & \text { Total } \\ \text { \& Wages: } & \text { \&Wages: } & \text { \&Wages: } & \text { Salaries } & \text { Operating } & \text { Library } \\ \text { Professional } & \text { Support } & \text { Student Asst. } & \text { \&Wages } & \text { Expenditures } & \text { Expenditures }\end{array}$

(17.a)

(17.b)

(17.c)

(17)

(19)

(20)

(Survey Question \#) INSTITUTION

\begin{tabular}{|c|c|c|c|c|c|c|}
\hline $3,177,458$ & $1,978,526$ & 641,720 & $5,797,704$ & $2,120,819$ & $14,868,525$ & ALABAMA \\
\hline $5,154,471$ & $7,851,683$ & 803,083 & $13,809,237$ & $2,063,189$ & $30,588,230$ & ALBERTA \\
\hline $5,789,522$ & $5,232,208$ & 733,363 & $11,755,093$ & $4,818,457$ & $28,333,910$ & ARIZONA \\
\hline $4,817,908$ & $6,068,037$ & 634,472 & $11,520,417$ & $2,528,302$ & $25,677,775$ & ARIZONA STATE \\
\hline $2,844,691$ & $1,370,986$ & 465,205 & $4,680,882$ & $2,283,891$ & $12,571,525$ & AUBURN \\
\hline $4,805,877$ & $3,244,192$ & 473,558 & $8,523,627$ & $2,113,448$ & $19,484,243$ & BOSTON \\
\hline $4,830,933$ & $2,650,794$ & 745,463 & $8,227,190$ & $1,106,872$ & $17,481,965$ & BOSTON COLLEGE \\
\hline $6,574,419$ & $1,856,251$ & $2,746,464$ & $11,177,134$ & $3,259,004$ & $23,942,505$ & BRIGHAM YOUNG \\
\hline $6,605,352$ & $6,462,502$ & 797,981 & $13,865,835$ & $2,859,571$ & $30,420,698$ & BRITISH COLUMBIA \\
\hline $4,290,618$ & $3,885,673$ & 471,819 & $8,648,110$ & $1,797,823$ & $18,945,391$ & BROWN \\
\hline $18,499,738$ & $9,819,252$ & $3,650,008$ & $31,968,998$ & $6,021,132$ & $56,186,972$ & CALIFORNIA, BERKELEY \\
\hline $4,081,533$ & $4,980,733$ & 693,545 & $9,755,811$ & $2,031,184$ & $20,609,936$ & CALIFORNIA, DAVIS \\
\hline $3,799,977$ & $4,242,846$ & 620,675 & $8,663,498$ & $1,297,937$ & $17,845,419$ & CALIFORNIA, IRVINE \\
\hline $11,778,277$ & $11,698,417$ & 2,794,295 & $26,270,989$ & $9,015,043$ & $50,919,689$ & CALIFORNIA, LOS ANGELES \\
\hline $3,223,850$ & $2,509,760$ & 520,201 & $6,253,811$ & $2,278,507$ & $14,888,349$ & CALIFORNIA, RIVERSIDE \\
\hline $5,662,505$ & $6,646,984$ & $1,261,080$ & $13,570,569$ & $4,529,147$ & $26,298,111$ & CALIFORNIA, SAN DIEGO \\
\hline $3,073,789$ & $4,135,856$ & $1,123,105$ & $8,332,750$ & $4,851,553$ & $18,580,864$ & CALIFORNIA, SANTA BARBARA \\
\hline $3,562,148$ & $1,762,779$ & 521,825 & $5,846,752$ & $2,597,127$ & $14,861,753$ & CASE WESTERN RESERVE \\
\hline $4,246,123$ & $6,150,494$ & $1,238,787$ & $11,635,404$ & $3,223,018$ & $31,671,892$ & CHICAGO \\
\hline $6,971,108$ & $2,339,287$ & 761,412 & $10,071,807$ & $2,552,039$ & $22,960,596$ & CINCINNATI \\
\hline $2,918,639$ & $4,485,540$ & 847,628 & $8,251,807$ & $1,380,087$ & $19,014,310$ & COLORADO \\
\hline $2,654,462$ & $2,111,044$ & 632,523 & $5,398,029$ & $1,670,566$ & $13,412,963$ & COLORADO STATE \\
\hline $19,000,096$ & $7,998,990$ & $2,058,310$ & $29,057,396$ & $6,223,006$ & $56,938,255$ & COLUMBIA \\
\hline $7,762,104$ & $5,154,758$ & 776,256 & $13,693,118$ & $2,171,752$ & $23,960,782$ & CONNECTICUT \\
\hline $7,942,102$ & $12,297,922$ & 956,705 & $21,196,729$ & $6,404,870$ & $42,156,480$ & CORNELL \\
\hline $3,876,818$ & $3,622,683$ & 441,425 & $7,940,926$ & $1,693,392$ & $17,497,174$ & DARTMOUTH \\
\hline $3,709,277$ & $3,099,240$ & 390,923 & $7,199,440$ & $1,647,113$ & $16,937,444$ & DELAWARE \\
\hline $9,317,351$ & $4,775,166$ & 505,765 & $14,598,282$ & $4,818,896$ & $33,531,731$ & DUKE \\
\hline $6,621,487$ & $5,117,896$ & 803,367 & $12,542,750$ & $4,007,780$ & $30,765,589$ & EMORY \\
\hline $6,102,471$ & $5,824,747$ & 411,916 & $12,339,134$ & $4,097,979$ & $27,435,482$ & FLORIDA \\
\hline $3,442,325$ & $3,038,262$ & 422,691 & $6,903,278$ & 460,142 & $15,083,949$ & FLORIDA STATE \\
\hline $4,899,650$ & $3,613,023$ & 635,583 & $9,148,256$ & $2,988,742$ & $21,727,764$ & GEORGE WASHINGTON \\
\hline $5,276,389$ & $4,241,267$ & 904,979 & $10,422,635$ & $2,440,663$ & $23,754,207$ & GEORGETOWN \\
\hline $3,778,959$ & $4,970,666$ & 737,712 & $9,487,337$ & $2,046,444$ & $23,014,039$ & GEORGIA \\
\hline $3,479,990$ & $1,235,173$ & 81,674 & $4,796,837$ & $1,232,259$ & $11,373,366$ & GEORGIA TECH \\
\hline $2,382,193$ & $2,577,585$ & 250,175 & $5,209,953$ & $1,136,966$ & $11,413,624$ & GUELPH \\
\hline $36,580,197$ & $19,745,688$ & $1,721,426$ & $58,047,311$ & $18,605,497$ & $105,809,085$ & HARVARD \\
\hline $3,703,901$ & $3,509,734$ & 913,273 & $8,126,908$ & $1,410,600$ & $17,306,904$ & HAWAII \\
\hline $3,075,546$ & $2,334,108$ & 500,493 & $5,910,147$ & $2,877,961$ & $17,000,792$ & HOUSTON \\
\hline $2,704,041$ & $1,886,625$ & 528,805 & $5,119,471$ & $1,263,805$ & $9,854,124$ & HOWARD \\
\hline $3,776,187$ & $3,568,928$ & 448,204 & $7,793,319$ & $1,861,703$ & $17,887,357$ & ILLINOIS, CHICAGO \\
\hline $10,464,305$ & $7,053,647$ & $1,633,373$ & $19,151,325$ & $4,639,760$ & $36,102,613$ & ILLINOIS, URBANA \\
\hline
\end{tabular}

UA/NA - Unavailable or Not Applicable 


\section{ARL LibRARY DATA TABLES, 2005-06 EXPENDITURES}

\begin{tabular}{|c|c|c|c|c|c|c|c|}
\hline $\begin{array}{l}\text { (Survey Question \#) } \\
\text { INSTITUTION }\end{array}$ & Notes & Monographs & $\begin{array}{r}\text { Current } \\
\text { Serials } \\
\\
(15 . b)\end{array}$ & $\begin{array}{r}\text { Other } \\
\text { Library } \\
\text { Materials } \\
\\
(15 . c)\end{array}$ & $\begin{array}{r}\text { Misc. } \\
\text { Materials }\end{array}$ & $\begin{array}{r}\text { Total } \\
\text { Library } \\
\text { Materials } \\
\\
(15)\end{array}$ & $\begin{array}{c}\text { Contract } \\
\text { Binding }\end{array}$ \\
\hline INDIANA & BL+ & $5,172,920$ & $7,326,574$ & $1,121,697$ & 286,637 & $13,907,828$ & 292,866 \\
\hline IOWA & GLM+ + & $2,266,663$ & $7,343,041$ & $2,451,342$ & 485,431 & $12,546,477$ & 172,921 \\
\hline IOWA STATE & $\mathrm{G}^{+}$ & $1,728,443$ & $6,539,217$ & 250,035 & 373,792 & $8,891,487$ & 189,125 \\
\hline JOHNS HOPKINS & BGM+ + & $2,566,064$ & $9,491,228$ & 860,738 & 720,658 & $13,638,688$ & 83,679 \\
\hline KANSAS & $\mathrm{LM}^{+}$ & $1,840,150$ & $5,483,771$ & 187,823 & 542,100 & $8,053,844$ & 119,987 \\
\hline KENT STATE & $\mathrm{G}^{+}$ & 663,496 & $2,940,211$ & UA/NA & 0 & $3,603,707$ & 77,460 \\
\hline KENTUCKY & GLM+ + & $1,459,327$ & $7,265,268$ & 223,809 & 515,109 & $9,463,513$ & 162,368 \\
\hline LAVAL & GLM+ & $2,149,866$ & $6,389,065$ & 90,138 & 172,415 & $8,801,485$ & 46,875 \\
\hline LOUISIANA STATE & LM+ & $1,685,235$ & $4,608,991$ & 381,623 & UA/NA & $6,675,849$ & 45,677 \\
\hline LOUISVILLE & GLM+ + & 863,090 & $8,076,155$ & 217,729 & 0 & $9,156,974$ & 100,398 \\
\hline MCGILL & GLM+ & $3,447,182$ & $6,699,174$ & $1,038,284$ & 365,313 & $11,549,953$ & 197,768 \\
\hline MCMASTER & GM & $1,344,741$ & $4,840,846$ & 421,874 & 221,825 & $6,829,286$ & 125,337 \\
\hline MANITOBA & GLM+ & $1,822,386$ & $4,528,145$ & 94,990 & 313,448 & $6,758,968$ & 132,052 \\
\hline MARYLAND & $\mathrm{G}^{+}$ & $1,974,070$ & $4,599,063$ & 289,063 & $1,835,944$ & $8,698,140$ & 130,867 \\
\hline MASSACHUSETTS & $\mathrm{G}^{+}$ & 974,487 & $4,339,742$ & 219,465 & 45,554 & $5,579,248$ & 88,436 \\
\hline MIT & $\mathrm{G}^{+}$ & $1,229,123$ & $6,324,816$ & 333,165 & 67,502 & $7,954,606$ & 195,044 \\
\hline MIAMI & GLM+ & $2,195,138$ & $8,946,033$ & 520,788 & 363,560 & $12,025,519$ & 138,811 \\
\hline MICHIGAN & $\mathrm{LM}^{+}$ & UA/NA & UA/NA & UA/NA & UA/NA & $20,669,495$ & UA/NA \\
\hline MICHIGAN STATE & BGL+ & $2,180,352$ & $7,295,789$ & 326,264 & 147,094 & $9,949,499$ & 215,132 \\
\hline MINNESOTA & BGLM+ + & $2,958,788$ & $9,859,444$ & $1,276,204$ & 62,736 & $14,157,172$ & 337,851 \\
\hline MISSOURI & BGLM+ + & $1,285,782$ & $4,989,546$ & 123,545 & 63,702 & $6,462,575$ & 161,493 \\
\hline MONTREAL & GLM+ & $1,960,987$ & $8,124,191$ & 100,271 & 175,841 & $10,361,290$ & 215,326 \\
\hline NEBRASKA & GL+ & $1,071,757$ & $5,386,159$ & 537,040 & 13,929 & $7,008,885$ & 155,410 \\
\hline NEW MEXICO & $\mathrm{LM}^{+}$ & 743,915 & $4,055,092$ & 97,141 & 409,560 & $5,305,708$ & 98,603 \\
\hline NEW YORK & GLM+ & $4,071,353$ & $10,900,902$ & $1,062,758$ & 104,121 & $16,139,134$ & 254,598 \\
\hline NORTH CAROLINA & GLM+ & $3,171,829$ & 8,151,067 & $2,663,591$ & 52,992 & $14,039,479$ & 222,197 \\
\hline NORTH CAROLINA STATE & $\mathrm{G}^{+}$ & $1,729,673$ & $5,813,216$ & 552,586 & 907,043 & $9,002,518$ & 194,319 \\
\hline NORTHWESTERN & GLM+ + & $3,347,394$ & $7,878,978$ & 389,596 & 549,635 & $12,165,603$ & 218,739 \\
\hline NOTRE DAME & GL+ & $3,363,219$ & $5,167,892$ & 431,401 & 577,785 & $9,540,297$ & 89,510 \\
\hline $\mathrm{OHIO}$ & BGM+ + & $1,198,802$ & $3,651,914$ & 347,099 & 129,462 & $5,327,277$ & 47,063 \\
\hline OHIO STATE & $\mathrm{LM}^{+}$ & $3,368,018$ & $6,953,106$ & $1,461,917$ & 422,898 & $12,205,939$ & 349,716 \\
\hline OKLAHOMA & GLM+ & $2,938,516$ & $6,136,135$ & $2,762,554$ & 281,181 & $12,118,386$ & 171,662 \\
\hline OKLAHOMA STATE & BGM & $\mathrm{UA} / \mathrm{NA}$ & UA/NA & UA/NA & UA/NA & $6,783,316$ & 47,039 \\
\hline OREGON & GL+ & $1,256,424$ & $3,070,990$ & UA/NA & 808,564 & $5,135,978$ & 101,297 \\
\hline PENNSYLVANIA & $\mathrm{LM}^{+}$ & $3,470,698$ & $10,005,808$ & 272,742 & UA/NA & $13,749,248$ & 254,238 \\
\hline PENNSYLVANIA STATE & GLM+ & $3,795,998$ & $12,249,954$ & $1,965,919$ & 77,006 & $18,088,877$ & 428,124 \\
\hline PITTSBURGH & GLM+ + & $3,155,615$ & $7,881,684$ & $2,138,375$ & 461,636 & $13,637,310$ & 221,850 \\
\hline PRINCETON & $\mathrm{B}+$ & $6,277,206$ & $7,480,441$ & $2,610,719$ & 386,474 & $16,754,840$ & 528,990 \\
\hline PURDUE & $\mathrm{G}^{+}$ & $2,216,481$ & $6,600,726$ & $2,156,864$ & 0 & $10,974,071$ & 111,164 \\
\hline QUEEN'S & BLM+ & $1,209,744$ & $6,114,253$ & 115,269 & 442,356 & $7,881,622$ & 86,281 \\
\hline RICE & $\mathrm{G}^{+}$ & $3,965,896$ & $5,295,592$ & 179,853 & 323,075 & $9,764,416$ & 61,582 \\
\hline ROCHESTER & BGM+ + & $1,054,356$ & $5,269,503$ & 345,683 & 46,045 & $6,715,587$ & 128,700 \\
\hline
\end{tabular}

UA/NA - Unavailable or Not Applicable

L - Includes Law library

M- Includes Medical library
+ - See Footnotes

$\mathrm{B}$ - Basis of volume count is bibliographic

G - Government documents not included in serials count 


\section{ARL LibRARY DATA TABLES, 2005-06 EXPENDITURES}

$\begin{array}{rrrrrr}\text { Salaries } & \text { Salaries } & \text { Salaries } & \text { Total } & \text { Other } & \text { Total } \\ \text { \& Wages: } & \text { \& Wages: } & \text { \&Wages: } & \text { Salaries } & \text { Operating } & \text { Library } \\ \text { Professional } & \text { Support } & \text { Student Asst. } & \text { \&Wages } & \text { Expenditures } & \text { Expenditures }\end{array}$

(17.a)

(17.b)

(17.c)

(17)

(19)

(20)

(Survey Question \#) INSTITUTION

\begin{tabular}{|c|c|c|c|c|c|c|}
\hline $8,031,434$ & $4,745,489$ & $1,536,062$ & $14,312,985$ & $3,629,712$ & $32,143,391$ & INDIANA \\
\hline $5,168,327$ & $5,113,295$ & $1,053,077$ & $11,334,699$ & $1,601,683$ & $25,655,780$ & IOWA \\
\hline $2,939,211$ & $3,486,981$ & 521,176 & $6,947,368$ & 945,544 & $16,973,524$ & IOWA STATE \\
\hline $5,517,577$ & $4,088,687$ & 541,698 & $10,147,962$ & $4,253,334$ & $28,123,663$ & JOHNS HOPKINS \\
\hline $5,706,192$ & $3,172,722$ & 892,097 & $9,771,011$ & $2,887,628$ & $20,832,470$ & KANSAS \\
\hline $2,729,004$ & $1,327,977$ & 384,007 & $4,440,988$ & $2,708,329$ & $10,830,484$ & KENT STATE \\
\hline $4,572,949$ & $2,545,302$ & 890,350 & $8,008,601$ & $1,934,664$ & $19,569,146$ & KENTUCKY \\
\hline $3,174,169$ & $4,864,493$ & 388,203 & $8,426,864$ & 419,986 & $17,695,209$ & LAVAL \\
\hline $2,860,137$ & $1,698,572$ & 538,835 & $5,097,544$ & $1,045,925$ & $12,864,995$ & LOUISIANA STATE \\
\hline $3,008,274$ & $2,429,149$ & 545,270 & $5,982,693$ & $3,253,551$ & $18,493,616$ & LOUISVILLE \\
\hline $4,767,665$ & $5,113,147$ & 620,752 & $10,501,564$ & $3,633,371$ & $25,882,656$ & MCGILL \\
\hline $1,855,687$ & $3,428,510$ & 347,088 & $5,631,285$ & $1,040,193$ & $13,626,100$ & MCMASTER \\
\hline $4,171,816$ & $4,542,770$ & 688,273 & $9,402,859$ & $2,940,720$ & $19,234,599$ & MANITOBA \\
\hline $6,667,609$ & $3,665,144$ & 862,340 & $11,195,093$ & $3,565,022$ & $23,589,122$ & MARYLAND \\
\hline $3,599,301$ & $3,105,989$ & 470,759 & $7,176,049$ & $1,836,714$ & $14,680,447$ & MASSACHUSETTS \\
\hline $7,347,676$ & $3,864,427$ & 362,616 & $11,574,719$ & $1,641,302$ & $21,365,671$ & MIT \\
\hline $4,701,108$ & $3,154,493$ & 590,947 & $8,446,548$ & $2,717,564$ & $23,328,442$ & MIAMI \\
\hline $11,085,528$ & $10,484,163$ & $1,634,060$ & $23,203,751$ & UA/NA & $49,053,402$ & MICHIGAN \\
\hline $4,565,393$ & $4,900,710$ & $1,430,509$ & $10,896,612$ & $3,047,831$ & $24,109,074$ & MICHIGAN STATE \\
\hline $7,165,391$ & $8,636,998$ & $1,667,146$ & $17,469,535$ & $6,357,109$ & $38,321,667$ & MINNESOTA \\
\hline $2,947,974$ & $2,660,908$ & 442,446 & $6,051,328$ & $1,879,906$ & $14,555,302$ & MISSOURI \\
\hline $6,174,250$ & $10,182,724$ & 86,423 & $16,443,397$ & $1,268,900$ & $28,288,912$ & MONTREAL \\
\hline $3,093,627$ & $2,870,517$ & 676,238 & $6,640,382$ & $2,334,020$ & $16,138,697$ & NEBRASKA \\
\hline $4,697,120$ & $5,738,191$ & 441,664 & $10,876,975$ & $3,116,758$ & $19,398,044$ & NEW MEXICO \\
\hline $8,059,700$ & $8,814,869$ & $1,311,054$ & $18,185,623$ & $6,424,257$ & $41,003,612$ & NEW YORK \\
\hline $9,192,948$ & $6,009,585$ & $1,000,390$ & $16,202,923$ & $4,024,002$ & $34,488,601$ & NORTH CAROLINA \\
\hline $6,352,335$ & $2,889,170$ & 791,917 & $10,033,422$ & $7,483,903$ & $26,714,162$ & NORTH CAROLINA STATE \\
\hline $6,921,463$ & $4,489,375$ & 656,808 & $12,067,646$ & $1,883,240$ & $26,335,228$ & NORTHWESTERN \\
\hline $4,544,693$ & $4,546,576$ & 448,309 & $9,539,578$ & $2,524,825$ & $21,694,210$ & NOTRE DAME \\
\hline $3,108,101$ & $2,250,373$ & 542,621 & $5,901,095$ & $2,356,964$ & $13,632,399$ & $\mathrm{OHIO}$ \\
\hline $6,940,002$ & $5,772,066$ & $2,442,543$ & $15,154,611$ & $5,255,845$ & $32,966,111$ & OHIO STATE \\
\hline $2,742,634$ & $2,288,062$ & 887,261 & $5,917,957$ & $1,648,347$ & $19,856,352$ & OKLAHOMA \\
\hline $3,319,561$ & $1,321,429$ & 649,305 & $5,290,295$ & $1,792,118$ & $13,912,768$ & OKLAHOMA STATE \\
\hline $3,039,434$ & $2,884,271$ & 997,480 & $6,921,185$ & $1,580,709$ & $13,739,169$ & OREGON \\
\hline $7,200,885$ & $6,816,157$ & $1,197,639$ & $15,214,681$ & $4,679,437$ & $33,897,604$ & PENNSYLVANIA \\
\hline $8,812,659$ & $13,913,369$ & 769,982 & $23,496,010$ & $6,567,041$ & $48,580,052$ & PENNSYLVANIA STATE \\
\hline $6,399,509$ & $4,608,125$ & 861,778 & $11,869,412$ & $3,198,640$ & $28,927,212$ & PITTSBURGH \\
\hline $9,038,404$ & $7,692,737$ & 908,198 & $17,639,339$ & $4,220,797$ & $39,143,966$ & PRINCETON \\
\hline $4,799,007$ & $4,354,254$ & 771,314 & $9,924,575$ & $3,014,032$ & $24,023,842$ & PURDUE \\
\hline $2,295,037$ & $3,835,646$ & 253,234 & $6,383,917$ & $1,907,213$ & $16,259,032$ & QUEEN'S \\
\hline $3,357,910$ & $1,428,647$ & 150,295 & $4,936,852$ & $1,146,717$ & $15,909,567$ & RICE \\
\hline $5,652,663$ & $2,151,492$ & 426,850 & $8,231,005$ & $2,336,792$ & $17,412,084$ & ROCHESTER \\
\hline
\end{tabular}

UA/NA - Unavailable or Not Applicable 


\section{ARL LibRARY DATA TABLES, 2005-06 EXPENDITURES}

\begin{tabular}{|c|c|c|c|c|c|c|c|}
\hline $\begin{array}{l}\text { (Survey Question \#) } \\
\text { INSTITUTION }\end{array}$ & Notes & Monographs & $\begin{array}{r}\text { Current } \\
\text { Serials } \\
\\
(15 . b)\end{array}$ & $\begin{array}{r}\text { Other } \\
\text { Library } \\
\text { Materials } \\
\\
(15 . c)\end{array}$ & $\begin{array}{r}\text { Misc. } \\
\text { Materials } \\
\\
\text { (15.d) }\end{array}$ & $\begin{array}{r}\text { Total } \\
\text { Library } \\
\text { Materials } \\
\\
\text { (15) }\end{array}$ & $\begin{array}{c}\text { Contract } \\
\text { Binding }\end{array}$ \\
\hline RUTGERS & GL+ & $1,496,784$ & $7,499,482$ & $1,158,654$ & 455,906 & $10,610,826$ & 140,994 \\
\hline SASKATCHEWAN & GLM+ + & $2,192,667$ & $5,407,965$ & UA/NA & 163,198 & $7,763,830$ & 72,293 \\
\hline SOUTH CAROLINA & BLM & $1,180,991$ & $5,717,091$ & 99,206 & 69,276 & $7,066,564$ & 100,052 \\
\hline SOUTHERN CALIFORNIA & GLM+ + & $3,059,117$ & $7,215,984$ & $2,572,207$ & 115,109 & $12,962,417$ & 132,861 \\
\hline SOUTHERN ILLINOIS & LM+ + & 804,712 & $5,698,153$ & 221,358 & 393,291 & $7,117,514$ & 133,893 \\
\hline SUNY-ALBANY & $\mathrm{G}^{+}$ & 881,550 & $4,030,434$ & 38,787 & 280,858 & $5,231,629$ & 84,653 \\
\hline SUNY-BUFFALO & GLM+ + & $1,033,042$ & $6,270,231$ & 286,315 & 175,949 & $7,765,537$ & 95,347 \\
\hline SUNY-STONY BROOK & $\mathrm{BM}+$ & 633,357 & $5,068,744$ & 200,270 & 688,357 & $6,590,728$ & 83,830 \\
\hline SYRACUSE & GL+ & $1,374,663$ & $4,444,275$ & 447,218 & 34,004 & $6,300,160$ & 60,615 \\
\hline TEMPLE & BLM+ & $2,106,159$ & $5,037,468$ & $1,600,214$ & 3,316 & $8,747,157$ & 111,907 \\
\hline TENNESSEE & GLM+ & $1,366,523$ & $9,261,449$ & 916,884 & 2,531 & $11,547,387$ & 132,888 \\
\hline TEXAS & L+ & $5,165,558$ & $8,610,285$ & $2,258,372$ & 794 & $16,035,009$ & 168,131 \\
\hline TEXAS A\&M & $\mathrm{G}^{+}$ & $4,363,873$ & $9,385,580$ & 326,862 & 23,166 & $14,099,481$ & 134,627 \\
\hline TEXAS TECH & BLM+ + & $1,898,119$ & $6,849,349$ & 195,882 & 560,179 & $9,503,529$ & 100,240 \\
\hline TORONTO & $\mathrm{GL}^{+}$ & $7,328,768$ & $10,010,684$ & $4,110,591$ & $\mathrm{UA} / \mathrm{NA}$ & $21,450,043$ & 398,653 \\
\hline TULANE & LM+ & $1,165,406$ & $5,039,744$ & $1,312,372$ & 157,953 & $7,675,475$ & 35,941 \\
\hline UTAH & BGLM+ & $1,768,974$ & $5,541,181$ & 65,887 & 113,824 & $7,489,866$ & 146,010 \\
\hline VANDERBILT & GLM+ & $2,022,886$ & $8,260,264$ & 294,663 & 0 & $10,577,813$ & 117,097 \\
\hline VIRGINIA & $\mathrm{LM}^{+}$ & $2,184,648$ & $6,741,512$ & 929,651 & 183,372 & $10,039,183$ & 82,638 \\
\hline VIRGINIA TECH & $\mathrm{G}^{+}$ & $1,412,630$ & $5,546,834$ & $\mathrm{UA} / \mathrm{NA}$ & 412,028 & $7,371,492$ & 43,183 \\
\hline WASHINGTON & $\mathrm{LM}+$ & $2,336,086$ & $9,818,882$ & 170,956 & 216,587 & $12,542,511$ & 245,692 \\
\hline WASHINGTON STATE & $\mathrm{G}^{+}$ & 983,879 & $4,453,232$ & 50,982 & 140,556 & $5,628,649$ & 73,020 \\
\hline WASHINGTON U.-ST. LOUIS & GLM+ + & $2,044,272$ & $7,275,964$ & $1,152,169$ & 174,058 & $10,646,463$ & 110,813 \\
\hline WATERLOO & $\mathrm{G}^{+}$ & $1,088,395$ & $4,540,404$ & 257,504 & 294,432 & $6,180,735$ & 62,982 \\
\hline WAYNE STATE & GLM+ & $1,225,350$ & $6,079,545$ & 0 & 506,711 & $7,811,606$ & 69,945 \\
\hline WESTERN ONTARIO & GL+ & $1,679,704$ & $7,490,733$ & UA/NA & 4,297 & $9,174,734$ & 108,715 \\
\hline WISCONSIN & GLM+ & $2,451,335$ & $6,629,327$ & $2,087,180$ & 414,868 & $11,582,710$ & 228,829 \\
\hline YALE & GLM+ & $12,088,699$ & $7,705,342$ & $14,119,514$ & 0 & $33,913,555$ & 588,915 \\
\hline YORK & $\mathrm{G}^{+}$ & $2,187,011$ & $6,057,219$ & 140,314 & $\mathrm{UA} / \mathrm{NA}$ & $8,384,544$ & 140,661 \\
\hline BOSTON PUBLIC & $\mathrm{G}^{+}$ & 3,971,455 & $1,020,617$ & $1,354,608$ & 5,528 & $6,352,208$ & 32,208 \\
\hline CISTI & $\mathrm{G}^{+}$ & 465,655 & $9,802,933$ & $1,280,860$ & 80,688 & $11,630,136$ & UA/NA \\
\hline CTR FOR RESEARCH LIBS & + & 54,563 & 307,084 & 531,229 & 6,657 & 899,533 & $\mathrm{UA} / \mathrm{NA}$ \\
\hline LIBRARY OF CONGRESS & BG+ & $4,280,822$ & $3,551,443$ & $4,408,946$ & 839,921 & $13,081,132$ & $1,502,682$ \\
\hline NATL AGRICULTURAL LIB & G+ & 208,202 & $4,375,151$ & 578,949 & 0 & $5,162,302$ & 0 \\
\hline LIB \& ARCHIVES CANADA & + & 735,047 & 881,794 & UA/NA & $\mathrm{UA} / \mathrm{NA}$ & $1,616,841$ & 6,789 \\
\hline NATL LIB OF MEDICINE & $\mathrm{G}^{+}$ & $1,189,678$ & $7,278,837$ & 161,297 & 0 & $8,629,812$ & 154,104 \\
\hline NEW YORK PUBLIC & G & $6,417,749$ & $4,500,054$ & $2,787,375$ & $\mathrm{UA} / \mathrm{NA}$ & $13,705,178$ & 550,569 \\
\hline NEW YORK STATE & + & $1,076,104$ & $2,587,943$ & 0 & 0 & $3,664,047$ & 25,785 \\
\hline SMITHSONIAN & + & 460,871 & $1,418,510$ & 4,321 & 179,647 & $2,063,349$ & 25,089 \\
\hline
\end{tabular}

UA/NA - Unavailable or Not Applicable

L - Includes Law library

M- Includes Medical library
+ - See Footnotes

$\mathrm{B}$ - Basis of volume count is bibliographic

G - Government documents not included in serials count 


\section{ARL LibRARY DATA TABLES, 2005-06 EXPENDITURES}

$\begin{array}{rrrrrr}\text { Salaries } & \text { Salaries } & \text { Salaries } & \text { Total } & \text { Other } & \text { Total } \\ \text { \&Wages: } & \text { \&Wages: } & \text { \&Wages: } & \text { Salaries } & \text { Operating } & \text { Library } \\ \text { Professional } & \text { Support } & \text { Student Asst. } & \text { \&Wages } & \text { Expenditures } & \text { Expenditures }\end{array}$

(17.a)

(17.b)

(17.c)

(17)

(19)

(20)

(Survey Question \#) INSTITUTION

\begin{tabular}{|c|c|c|c|c|c|c|}
\hline $6,940,144$ & $9,892,372$ & $1,425,148$ & $18,257,664$ & $2,244,119$ & $31,253,603$ & RUTGERS \\
\hline $2,879,334$ & $2,734,527$ & 265,111 & $5,878,972$ & $1,114,615$ & $14,829,710$ & SASKATCHEWAN \\
\hline $3,972,269$ & $3,386,975$ & 477,933 & $7,837,177$ & $5,418,559$ & $20,422,352$ & SOUTH CAROLINA \\
\hline $9,191,567$ & $3,392,887$ & $1,949,739$ & $14,534,193$ & $4,264,218$ & $31,893,689$ & SOUTHERN CALIFORNIA \\
\hline $2,793,840$ & $2,749,931$ & 998,870 & $6,542,641$ & 825,601 & $14,619,649$ & SOUTHERN ILLINOIS \\
\hline $3,432,271$ & $2,160,600$ & 518,768 & $6,111,639$ & 613,972 & $12,041,893$ & SUNY-ALBANY \\
\hline $7,522,953$ & $1,468,156$ & 973,650 & $9,964,759$ & $1,628,145$ & $19,453,788$ & SUNY-BUFFALO \\
\hline $4,983,702$ & 829,702 & 493,406 & $6,306,810$ & $1,133,427$ & $14,114,795$ & SUNY-STONY BROOK \\
\hline $3,956,441$ & $3,923,591$ & 360,308 & $8,240,340$ & $1,235,583$ & $15,836,698$ & SYRACUSE \\
\hline $4,326,009$ & $2,178,223$ & 674,351 & $7,178,583$ & $1,835,712$ & $17,873,359$ & TEMPLE \\
\hline $5,087,741$ & $3,664,349$ & 682,622 & $9,434,712$ & $1,756,502$ & $22,871,489$ & TENNESSEE \\
\hline $8,471,146$ & $9,815,712$ & $1,523,455$ & $19,810,313$ & $5,572,367$ & $41,585,820$ & TEXAS \\
\hline $6,758,496$ & $3,401,115$ & 917,856 & $11,077,467$ & $4,200,120$ & $29,511,695$ & TEXAS A\&M \\
\hline $4,394,293$ & $2,832,482$ & $1,004,723$ & $8,231,498$ & $3,489,492$ & $21,324,759$ & TEXAS TECH \\
\hline $12,495,097$ & $13,894,504$ & $3,431,991$ & $29,821,592$ & $5,940,464$ & $57,610,752$ & TORONTO \\
\hline $2,828,106$ & $2,499,251$ & 292,961 & $5,620,318$ & 929,771 & $14,261,505$ & TULANE \\
\hline $4,174,358$ & $5,579,958$ & $1,428,575$ & $11,182,891$ & $4,469,965$ & $23,288,732$ & UTAH \\
\hline $5,567,319$ & $3,089,867$ & 346,032 & $9,003,218$ & $3,308,978$ & $23,007,106$ & VANDERBILT \\
\hline $8,467,566$ & $10,533,160$ & $1,203,781$ & $20,204,507$ & $4,448,759$ & $34,775,087$ & VIRGINIA \\
\hline $2,134,226$ & $2,648,245$ & 449,684 & $5,232,155$ & $1,436,106$ & $14,082,936$ & VIRGINIA TECH \\
\hline $10,867,476$ & $7,432,506$ & $2,266,984$ & $20,566,966$ & $5,486,661$ & $38,841,830$ & WASHINGTON \\
\hline $2,827,045$ & $2,824,690$ & 616,959 & $6,268,694$ & $1,573,838$ & $13,544,201$ & WASHINGTON STATE \\
\hline $7,863,346$ & $4,297,528$ & 762,306 & $12,923,180$ & $7,786,020$ & $31,466,476$ & WASHINGTON U.-ST. LOUIS \\
\hline $2,124,399$ & $3,221,459$ & 654,919 & $6,000,777$ & $2,593,655$ & $14,838,150$ & WATERLOO \\
\hline $4,704,501$ & $2,810,915$ & $1,297,054$ & $8,812,470$ & $4,370,981$ & $21,065,002$ & WAYNE STATE \\
\hline $3,515,827$ & $3,123,903$ & 333,011 & $6,972,741$ & 751,520 & $17,007,710$ & WESTERN ONTARIO \\
\hline $13,380,066$ & $6,259,109$ & $2,741,556$ & $22,380,731$ & $6,607,997$ & $40,800,267$ & WISCONSIN \\
\hline $17,565,125$ & $12,516,930$ & $1,222,416$ & $31,304,471$ & $9,131,178$ & $74,938,119$ & YALE \\
\hline $4,247,205$ & $4,202,457$ & 995,194 & $9,444,855$ & $1,129,115$ & $19,099,174$ & YORK \\
\hline $10,916,229$ & $11,118,952$ & 557,365 & $22,592,546$ & $9,342,206$ & $38,319,168$ & BOSTON PUBLIC \\
\hline UA/NA & UA/NA & UA/NA & $16,327,578$ & $12,009,245$ & $39,966,959$ & CISTI \\
\hline $1,363,864$ & 440,392 & 274,040 & $2,078,296$ & 652,345 & $3,630,174$ & CTR FOR RESEARCH LIBS \\
\hline $305,666,000$ & $\mathrm{UA} / \mathrm{NA}$ & $\mathrm{UA} / \mathrm{NA}$ & $305,666,000$ & $296,733,185$ & $616,982,999$ & LIBRARY OF CONGRESS \\
\hline $11,643,768$ & $2,857,341$ & 118,374 & $14,619,483$ & $9,886,541$ & $29,668,326$ & NATL AGRICULTURAL LIB \\
\hline UA/NA & UA/NA & UA/NA & $\mathrm{UA} / \mathrm{NA}$ & $\mathrm{UA} / \mathrm{NA}$ & $\mathrm{UA} / \mathrm{NA}$ & LIB \& ARCHIVES CANADA \\
\hline $15,933,857$ & $3,451,782$ & 509,361 & $19,895,000$ & $33,609,084$ & $62,288,000$ & NATL LIB OF MEDICINE \\
\hline $27,855,568$ & $\mathrm{UA} / \mathrm{NA}$ & $1,437,441$ & $29,293,009$ & $8,482,459$ & $52,031,215$ & NEW YORK PUBLIC \\
\hline UA/NA & $\mathrm{UA} / \mathrm{NA}$ & UA/NA & $7,720,699$ & $1,063,835$ & $12,474,366$ & NEW YORK STATE \\
\hline $4,457,701$ & $2,049,554$ & 9,331 & $6,516,586$ & $2,422,324$ & $11,027,348$ & SMITHSONIAN \\
\hline
\end{tabular}

UA/NA - Unavailable or Not Applicable 


\section{ARL LibRARY DATA TABLES, 2005-06 \\ SUMMARY DATA: EXPENDITURES}

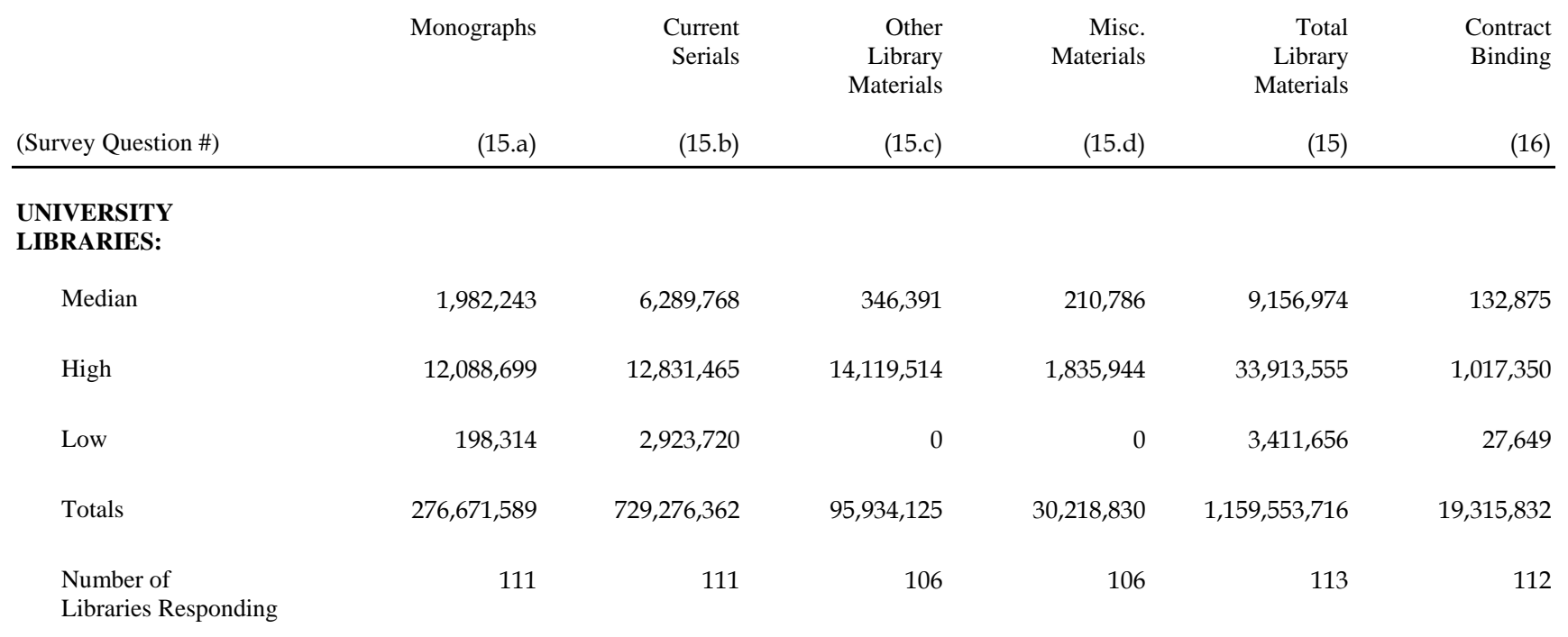

\section{NONUNIVERSITY}

LIBRARIES:

Median
Totals
Number of
Libraries Responding

$\begin{array}{rrrrrr}905,576 & 3,069,693 & 578,949 & 6,093 & 5,757,255 & 28,997 \\ 18,860,147 & 35,724,366 & 11,107,585 & 1,112,441 & 66,804,538 & 2,297,226 \\ 10 & 10 & 9 & 8 & 10 & 8\end{array}$

GRAND TOTALS

$765,000,727 \quad 107,041,709$

$31,331,271$

$1,226,358,254$

$21,613,058$ 


\section{ARL LIBRARY DATA TABLES, 2005-06}

SUMMARY DATA: EXPENDITURES

\begin{tabular}{|c|c|c|c|c|c|c|}
\hline $\begin{array}{r}\text { Salaries } \\
\text { \& Wages: } \\
\text { Professional } \\
\\
\text { (17.a) }\end{array}$ & $\begin{array}{r}\text { Salaries } \\
\text { \& Wages: } \\
\text { Support } \\
(17 . b)\end{array}$ & $\begin{array}{r}\text { Salaries } \\
\text { \& Wages: } \\
\text { Student Asst. } \\
\text { (17.c) }\end{array}$ & $\begin{array}{r}\text { Total } \\
\text { Salaries } \\
\text { \& Wages } \\
(17)\end{array}$ & $\begin{array}{r}\text { Other } \\
\text { Operating } \\
\text { Expenditures } \\
(19)\end{array}$ & $\begin{array}{r}\text { Total } \\
\text { Library } \\
\text { Expenditures } \\
(20)\end{array}$ & (Survey Question \#) \\
\hline & & & & & & $\begin{array}{l}\text { UNIVERSITY } \\
\text { LIBRARIES: }\end{array}$ \\
\hline $4,704,501$ & $3,835,646$ & 693,545 & $9,444,855$ & $2,526,564$ & $21,324,759$ & Median \\
\hline $36,580,197$ & $19,745,688$ & $3,650,008$ & $58,047,311$ & $18,605,497$ & $105,809,085$ & High \\
\hline $1,855,687$ & 829,702 & 81,674 & $4,440,988$ & 419,986 & $9,854,124$ & Low \\
\hline $663,055,019$ & $536,402,618$ & $101,289,643$ & $1,300,747,280$ & $353,830,130$ & $2,838,627,114$ & Totals \\
\hline 113 & 113 & 113 & 113 & 112 & 113 & $\begin{array}{r}\text { Number of } \\
\text { Libraries Responding }\end{array}$ \\
\hline & & & & & & $\begin{array}{r}\text { NONUNIVERSITY } \\
\text { LIBRARIES: }\end{array}$ \\
\hline $11,643,768$ & $2,857,341$ & 391,701 & $16,327,578$ & $9,342,206$ & $38,319,168$ & Median \\
\hline $377,836,987$ & $19,918,021$ & $2,905,912$ & $424,709,197$ & $374,201,224$ & $866,388,555$ & Totals \\
\hline 7 & 5 & 6 & 9 & 9 & 9 & $\begin{array}{r}\text { Number of } \\
\text { Libraries Responding }\end{array}$ \\
\hline $1,040,892,006$ & $556,320,639$ & $104,195,555$ & $1,725,456,477$ & $728,031,354$ & $3,705,015,669$ & GRAND TOTALS \\
\hline
\end{tabular}




\section{ARL LiBRARY DATA TABLES, 2005-06 EXPENDITURES FOR ELECTRONIC RESOURCES}

\begin{tabular}{|c|c|c|c|c|c|c|}
\hline $\begin{array}{l}\text { (Survey Question \#) } \\
\text { INSTITUTION }\end{array}$ & Notes & $\begin{array}{r}\text { Computer } \\
\text { Files } \\
\\
(21)\end{array}$ & $\begin{array}{r}\text { Electronic } \\
\text { Serials } \\
\\
(22)\end{array}$ & $\begin{array}{r}\text { Total } \\
\text { E-Materials } \\
\\
(21+22)\end{array}$ & $\begin{array}{r}\text { Total } \\
\text { Materials } \\
\\
\text { (15) }\end{array}$ & $\begin{array}{l}\text { E-Materials As \% } \\
\text { Of Lib. Materials }\end{array}$ \\
\hline ALABAMA & BGLM+ & 421,390 & $3,156,885$ & $3,578,275$ & $6,870,657$ & 52.08 \\
\hline ALBERTA & $\mathrm{GL}^{+}$ & 842,997 & $6,163,153$ & $7,006,150$ & $14,590,484$ & 48.02 \\
\hline ARIZONA & BGLM+ & 486,983 & $5,073,930$ & $5,560,913$ & $11,662,200$ & 47.68 \\
\hline ARIZONA STATE & GL+ & 138,996 & $4,871,334$ & $5,010,330$ & $11,474,442$ & 43.67 \\
\hline AUBURN & BG+ + & 124,193 & $2,351,968$ & $2,476,161$ & $5,543,726$ & 44.67 \\
\hline BOSTON & GLM+ & 0 & $1,716,862$ & $1,716,862$ & $8,707,614$ & 19.72 \\
\hline BOSTON COLLEGE & GL+ & UA/NA & $1,700,151$ & $1,700,151$ & $8,009,522$ & 21.23 \\
\hline BRIGHAM YOUNG & BGL+ & UA/NA & $1,981,220$ & $1,981,220$ & $9,271,776$ & 21.37 \\
\hline BRITISH COLUMBIA & GLM+ + & 275,246 & $6,448,284$ & $6,723,530$ & $13,523,834$ & 49.72 \\
\hline BROWN & $\mathrm{M}+$ & 26,875 & $3,316,936$ & $3,343,811$ & $8,285,394$ & 40.36 \\
\hline CALIFORNIA, BERKELEY & $\mathrm{L}^{+}$ & UA/NA & UA/NA & UA/NA & $17,453,180$ & UA/NA \\
\hline CALIFORNIA, DAVIS & GLM+ + & 992,683 & $2,800,190$ & $3,792,873$ & $8,659,069$ & 43.80 \\
\hline CALIFORNIA, IRVINE & BGM+ & 414,341 & $2,996,839$ & $3,411,180$ & $7,814,726$ & 43.65 \\
\hline CALIFORNIA, LOS ANGELES & $\mathrm{LM}+$ & 351,134 & $2,936,404$ & $3,287,538$ & $15,158,149$ & 21.69 \\
\hline CALIFORNIA, RIVERSIDE & BG & 68,521 & $2,840,171$ & $2,908,692$ & $6,109,872$ & 47.61 \\
\hline CALIFORNIA, SAN DIEGO & $\mathrm{M}+$ & 80,005 & $3,359,237$ & $3,439,242$ & $7,922,917$ & 43.41 \\
\hline CALIFORNIA, SANTA BARBARA & & 1,405 & $2,247,078$ & $2,248,483$ & $5,236,330$ & 42.94 \\
\hline CASE WESTERN RESERVE & GLM+ + & 0 & $2,866,823$ & $2,866,823$ & $6,304,139$ & 45.48 \\
\hline CHICAGO & BGLM+ + & $2,000,839$ & $4,020,208$ & $6,021,047$ & $16,462,379$ & 36.57 \\
\hline CINCINNATI & GLM+ & 630,775 & $6,609,189$ & $7,239,964$ & $10,209,066$ & 70.92 \\
\hline COLORADO & BG+ & 619,806 & $4,559,419$ & $5,179,225$ & $9,315,863$ & 55.60 \\
\hline COLORADO STATE & $\mathrm{G}^{+}$ & 41,302 & $2,994,932$ & $3,036,234$ & $6,265,271$ & 48.46 \\
\hline COLUMBIA & $\mathrm{LM}+$ & $1,309,495$ & $6,395,193$ & $7,704,688$ & $21,017,791$ & 36.66 \\
\hline CONNECTICUT & GLM+ & 159,597 & $3,344,931$ & $3,504,528$ & $7,854,879$ & 44.62 \\
\hline CORNELL & GL+ & 33,661 & $5,332,781$ & $5,366,442$ & $14,381,579$ & 37.31 \\
\hline DARTMOUTH & GM+ & 214,066 & $3,949,911$ & $4,163,977$ & $7,748,473$ & 53.74 \\
\hline DELAWARE & $\mathrm{G}^{+}$ & 365,567 & $4,545,072$ & $4,910,639$ & $8,005,686$ & 61.34 \\
\hline DUKE & BLM+ + & 398,017 & $4,065,322$ & $4,463,339$ & $13,961,002$ & 31.97 \\
\hline EMORY & $\mathrm{LM}+$ & 66,959 & $2,868,246$ & $2,935,205$ & $14,083,609$ & 20.84 \\
\hline FLORIDA & $\mathrm{LM}+$ & 138,162 & $5,217,474$ & $5,355,636$ & $10,821,088$ & 49.49 \\
\hline FLORIDA STATE & BLM+ + & $1,163,192$ & $2,717,648$ & $3,880,840$ & $7,650,054$ & 50.73 \\
\hline GEORGE WASHINGTON & BLM+ + & 510,874 & $2,384,056$ & $2,894,930$ & $9,457,211$ & 30.61 \\
\hline GEORGETOWN & BGLM+ + & 471,062 & $2,643,690$ & $3,114,752$ & $10,783,633$ & 28.88 \\
\hline GEORGIA & GL+ & 343,740 & $5,135,204$ & $5,478,944$ & $11,217,811$ & 48.84 \\
\hline GEORGIA TECH & $\mathrm{G}^{+}$ & $\mathrm{UA} / \mathrm{NA}$ & $\mathrm{UA} / \mathrm{NA}$ & $\mathrm{UA} / \mathrm{NA}$ & $5,306,847$ & $\mathrm{UA} / \mathrm{NA}$ \\
\hline GUELPH & $\mathrm{G}^{+}$ & 251,375 & $2,947,304$ & $3,198,679$ & $5,039,056$ & 63.48 \\
\hline HARVARD & GLM+ + & $5,417,996$ & UA/NA & $5,417,996$ & $28,138,927$ & 19.25 \\
\hline HAWAII & $\mathrm{L}+$ & UA/NA & $2,471,454$ & $2,471,454$ & 7,611,078 & 32.47 \\
\hline HOUSTON & BGL+ + & 206,428 & $2,171,667$ & $2,378,095$ & $8,117,193$ & 29.30 \\
\hline HOWARD & BLM+ & 0 & $1,070,931$ & $1,070,931$ & $3,411,656$ & 31.39 \\
\hline ILLINOIS, CHICAGO & GM+ & UA/NA & $3,455,796$ & $3,455,796$ & $8,166,623$ & 42.32 \\
\hline ILLINOIS, URBANA & GL+ & 28,444 & $3,218,605$ & $3,247,049$ & $12,043,672$ & 26.96 \\
\hline
\end{tabular}

UA/NA - Unavailable or Not Applicable

L - Includes Law library

M- Includes Medical library
+ - See Footnotes

B - Basis of volume count is bibliographic

G - Government documents not included in serials count 


\section{ARL LibRARY DATA TABLES, 2005-06 EXPENDITURES FOR ELECTRONIC RESOURCES}

Bibl. Utilities, Networks, and Consortia

Library

(23.a)

External

(23.b)

And Software

(24)
Document Delivery/ Interlibrary Loan

(25)
(Survey Question \#) INSTITUTION

\begin{tabular}{|c|c|c|c|c|}
\hline 228,260 & UA/NA & 505,594 & 36,115 & ALABAMA \\
\hline UA/NA & UA/NA & UA/NA & UA/NA & ALBERTA \\
\hline $\mathrm{UA} / \mathrm{NA}$ & UA/NA & 633,360 & 172,395 & ARIZONA \\
\hline 170,359 & 0 & 676,587 & 261,710 & ARIZONA STATE \\
\hline 152,709 & 150,000 & 575,921 & 136,451 & AUBURN \\
\hline 255,700 & 2,500 & 373,469 & 70,842 & BOSTON \\
\hline 170,553 & UA/NA & 148,903 & 8,136 & BOSTON COLLEGE \\
\hline 467,579 & UA/NA & 606,093 & 65,376 & BRIGHAM YOUNG \\
\hline 44,232 & 0 & UA/NA & 162,573 & BRITISH COLUMBIA \\
\hline 195,841 & 41,479 & 318,495 & 150,548 & BROWN \\
\hline UA/NA & UA/NA & UA/NA & UA/NA & CALIFORNIA, BERKELEY \\
\hline 226,960 & 989,069 & 556,769 & 144,588 & CALIFORNIA, DAVIS \\
\hline 118,614 & 837,416 & 425,485 & 71,770 & CALIFORNIA, IRVINE \\
\hline 464,838 & $1,252,969$ & $2,678,825$ & 280,909 & CALIFORNIA, LOS ANGELES \\
\hline 173,566 & UA/NA & 887,870 & 49,796 & CALIFORNIA, RIVERSIDE \\
\hline 0 & 880,388 & $1,270,277$ & 210,781 & CALIFORNIA, SAN DIEGO \\
\hline 264,000 & 0 & 569,489 & 80,357 & CALIFORNIA, SANTA BARBARA \\
\hline 38,491 & 137,298 & 335,920 & 32,545 & CASE WESTERN RESERVE \\
\hline 330,075 & 250,000 & 503,909 & 186,740 & CHICAGO \\
\hline 138,929 & 464,500 & $1,046,140$ & 25,963 & CINCINNATI \\
\hline 347,370 & 28,026 & 193,005 & 121,280 & COLORADO \\
\hline 256,865 & 296,559 & 653,864 & 279,283 & COLORADO STATE \\
\hline 485,143 & 0 & $1,238,933$ & 11,271 & COLUMBIA \\
\hline 424,538 & $1,894,324$ & 340,765 & 153,995 & CONNECTICUT \\
\hline 184,768 & 0 & $1,585,474$ & 148,271 & CORNELL \\
\hline UA/NA & 0 & 174,867 & 122,274 & DARTMOUTH \\
\hline 129,979 & 0 & 501,621 & 32,690 & DELAWARE \\
\hline 130,754 & 6,254 & 90,109 & 10,063 & DUKE \\
\hline 289,632 & 277,845 & 316,041 & 68,091 & EMORY \\
\hline 11,202 & 0 & 327,713 & 23,431 & FLORIDA \\
\hline 224,112 & 514,167 & 222,821 & 6,721 & FLORIDA STATE \\
\hline 729,645 & 89,485 & 618,080 & 214,634 & GEORGE WASHINGTON \\
\hline 166,404 & UA/NA & 502,397 & 45,567 & GEORGETOWN \\
\hline 116,491 & 0 & 404,481 & 431 & GEORGIA \\
\hline 50,000 & UA/NA & 397,273 & 237,845 & GEORGIA TECH \\
\hline 130,965 & 0 & 483,942 & 185,834 & GUELPH \\
\hline $2,111,812$ & $\mathrm{UA} / \mathrm{NA}$ & $1,548,540$ & 49,205 & HARVARD \\
\hline 142,599 & 0 & 142,362 & 70,000 & HAWAII \\
\hline 410,871 & 0 & 714,564 & 34,850 & HOUSTON \\
\hline 184,585 & 2 & 61,839 & 24,704 & HOWARD \\
\hline UA/NA & UA/NA & $\mathrm{UA} / \mathrm{NA}$ & UA/NA & ILLINOIS, CHICAGO \\
\hline 280,847 & UA/NA & 406,216 & 28,883 & ILLINOIS, URBANA \\
\hline
\end{tabular}




\section{ARL LiBRARY DATA TABLES, 2005-06 EXPENDITURES FOR ELECTRONIC RESOURCES}

\begin{tabular}{|c|c|c|c|c|c|c|}
\hline $\begin{array}{l}\text { (Survey Question \#) } \\
\text { INSTITUTION }\end{array}$ & Notes & $\begin{array}{r}\text { Computer } \\
\text { Files } \\
\\
(21)\end{array}$ & $\begin{array}{r}\text { Electronic } \\
\text { Serials } \\
\\
(22)\end{array}$ & $\begin{array}{r}\text { Total } \\
\text { E-Materials } \\
\\
(21+22)\end{array}$ & $\begin{array}{r}\text { Total } \\
\text { Materials } \\
\\
(15)\end{array}$ & $\begin{array}{l}\text { E-Materials As \% } \\
\text { Of Lib. Materials }\end{array}$ \\
\hline INDIANA & BL+ & 571,106 & $3,563,997$ & $4,135,103$ & $13,907,828$ & 29.73 \\
\hline IOWA & GLM+ + & 614,710 & $4,806,427$ & $5,421,137$ & $12,546,477$ & 43.21 \\
\hline IOWA STATE & $\mathrm{G}^{+}$ & 248,180 & $5,742,838$ & $5,991,018$ & $8,891,487$ & 67.38 \\
\hline JOHNS HOPKINS & BGM+ & 44,414 & $6,445,836$ & $6,490,250$ & $13,638,688$ & 47.59 \\
\hline KANSAS & $\mathrm{LM}+$ & 103,696 & $2,330,660$ & $2,434,356$ & $8,053,844$ & 30.23 \\
\hline KENT STATE & $\mathrm{G}^{+}$ & 22,266 & $1,710,221$ & $1,732,487$ & $3,603,707$ & 48.08 \\
\hline KENTUCKY & GLM+ + & 654,843 & $2,836,716$ & $3,491,559$ & $9,463,513$ & 36.89 \\
\hline LAVAL & GLM+ & 502,461 & $3,821,109$ & $4,323,571$ & $8,801,485$ & 49.12 \\
\hline LOUISIANA STATE & $\mathrm{LM}+$ & 328,935 & $1,141,396$ & $1,470,331$ & $6,675,849$ & 22.02 \\
\hline LOUISVILLE & GLM+ & 0 & $2,703,346$ & $2,703,346$ & $9,156,974$ & 29.52 \\
\hline MCGILL & GLM+ & 614,648 & $5,402,199$ & $6,016,847$ & $11,549,953$ & 52.09 \\
\hline MCMASTER & GM & 635,261 & $3,535,263$ & $4,170,524$ & $6,829,286$ & 61.07 \\
\hline MANITOBA & GLM+ & 478,179 & $2,672,829$ & $3,151,007$ & $6,758,968$ & 46.62 \\
\hline MARYLAND & $\mathrm{G}^{+}$ & 62,800 & $3,876,605$ & $3,939,405$ & $8,698,140$ & 45.29 \\
\hline MASSACHUSETTS & $\mathrm{G}^{+}$ & 0 & $2,637,996$ & $2,637,996$ & $5,579,248$ & 47.28 \\
\hline MIT & $\mathrm{G}^{+}$ & 350,311 & $2,895,014$ & $3,245,325$ & $7,954,606$ & 40.80 \\
\hline MIAMI & GLM+ & 490,000 & $3,354,486$ & $3,844,486$ & $12,025,519$ & 31.97 \\
\hline MICHIGAN & $\mathrm{LM}+$ & $\mathrm{UA} / \mathrm{NA}$ & UA/NA & $\mathrm{UA} / \mathrm{NA}$ & $20,669,495$ & $\mathrm{UA} / \mathrm{NA}$ \\
\hline MICHIGAN STATE & BGL+ & 567,730 & $4,332,235$ & $4,899,965$ & $9,949,499$ & 49.25 \\
\hline MINNESOTA & BGLM+ & 468,873 & $4,945,954$ & $5,414,827$ & $14,157,172$ & 38.25 \\
\hline MISSOURI & BGLM+ & 84,016 & $2,703,951$ & $2,787,967$ & $6,462,575$ & 43.14 \\
\hline MONTREAL & GLM+ & 380,367 & $4,649,791$ & $5,030,158$ & $10,361,290$ & 48.55 \\
\hline NEBRASKA & GL+ & 450,030 & $1,665,456$ & $2,115,486$ & $7,008,885$ & 30.18 \\
\hline NEW MEXICO & $\mathrm{LM}+$ & 236,161 & $1,969,433$ & $2,205,594$ & $5,305,708$ & 41.57 \\
\hline NEW YORK & GLM+ & $1,000,152$ & $5,688,115$ & $6,688,267$ & $16,139,134$ & 41.44 \\
\hline NORTH CAROLINA & GLM+ + & 900,041 & $2,904,753$ & $3,804,794$ & $14,039,479$ & 27.10 \\
\hline NORTH CAROLINA STATE & $\mathrm{G}^{+}$ & 586,318 & $1,819,223$ & $2,405,541$ & $9,002,518$ & 26.72 \\
\hline NORTHWESTERN & GLM+ & $1,287,769$ & $4,556,562$ & $5,844,331$ & $12,165,603$ & 48.04 \\
\hline NOTRE DAME & GL+ & 649,750 & $3,565,174$ & $4,214,924$ & $9,540,297$ & 44.18 \\
\hline OHIO & BGM+ & 129,937 & $2,296,048$ & $2,425,985$ & $5,327,277$ & 45.54 \\
\hline OHIO STATE & $\mathrm{LM}+$ & 158,950 & $5,015,744$ & $5,174,694$ & $12,205,939$ & 42.39 \\
\hline OKLAHOMA & GLM+ & $1,153,070$ & $1,759,791$ & $2,912,861$ & $12,118,386$ & 24.04 \\
\hline OKLAHOMA STATE & BGM & UA/NA & UA/NA & UA/NA & $6,783,316$ & UA/NA \\
\hline OREGON & GL+ & 84,000 & $1,679,019$ & $1,763,019$ & $5,135,978$ & 34.33 \\
\hline PENNSYLVANIA & $\mathrm{LM}+$ & 121,368 & $5,109,714$ & $5,231,082$ & $13,749,248$ & 38.05 \\
\hline PENNSYLVANIA STATE & GLM+ & $1,665,252$ & $8,158,340$ & $9,823,592$ & $18,088,877$ & 54.31 \\
\hline PITTSBURGH & GLM+ & 302,529 & $5,589,092$ & $5,891,621$ & $13,637,310$ & 43.20 \\
\hline PRINCETON & $\mathrm{B}+$ & $2,677,193$ & $2,265,019$ & $4,942,212$ & $16,754,840$ & 29.50 \\
\hline PURDUE & $\mathrm{G}^{+}$ & 0 & $2,017,160$ & $2,017,160$ & $10,974,071$ & 18.38 \\
\hline QUEEN'S & BLM+ + & $\mathrm{UA} / \mathrm{NA}$ & $4,478,343$ & $4,478,343$ & $7,881,622$ & 56.82 \\
\hline RICE & $\mathrm{G}^{+}$ & $1,130,638$ & $2,920,098$ & $4,050,736$ & $9,764,416$ & 41.48 \\
\hline ROCHESTER & BGM+ & 22,065 & $1,693,928$ & $1,715,993$ & $6,715,587$ & 25.55 \\
\hline
\end{tabular}

UA/NA - Unavailable or Not Applicable

L - Includes Law library

M- Includes Medical library
+ - See Footnotes

B - Basis of volume count is bibliographic

G - Government documents not included in serials count 


\section{ARL LibRARY DATA TABLES, 2005-06 EXPENDITURES FOR ELECTRONIC RESOURCES}

Bibl. Utilities, Networks, and Consortia

Library

(23.a)

(23.b)

\begin{tabular}{|c|c|c|c|c|}
\hline 108,122 & 229,540 & 539,189 & 187,382 & INDIANA \\
\hline 298,248 & 0 & 380,026 & 257,653 & IOWA \\
\hline 90,485 & 0 & 331,920 & 133,298 & IOWA STATE \\
\hline 262,643 & 479 & 491,896 & 232,871 & JOHNS HOPKINS \\
\hline 394,148 & 0 & 771,953 & 259,815 & KANSAS \\
\hline 0 & 543,973 & 360,557 & 16,184 & KENT STATE \\
\hline 200,000 & 0 & 554,544 & 179,694 & KENTUCKY \\
\hline 174,499 & 0 & 366,361 & 95,407 & LAVAL \\
\hline 297,974 & 109,757 & 87,193 & 174,955 & LOUISIANA STATE \\
\hline 204,114 & 6,000 & 403,364 & 35,805 & LOUISVILLE \\
\hline 186,433 & UA/NA & 696,338 & 145,078 & MCGILL \\
\hline 107,055 & 0 & 315,053 & 94,539 & MCMASTER \\
\hline UA/NA & UA/NA & 347,514 & 164,355 & MANITOBA \\
\hline 68,832 & UA/NA & 817,658 & 370,551 & MARYLAND \\
\hline 128,515 & 75,000 & 258,351 & 109,895 & MASSACHUSETTS \\
\hline 93,144 & 0 & 387,156 & 96,525 & MIT \\
\hline 223,425 & 0 & 791,099 & 132,858 & MIAMI \\
\hline UA/NA & UA/NA & UA/NA & UA/NA & MICHIGAN \\
\hline 235,686 & UA/NA & 663,450 & 268,920 & MICHIGAN STATE \\
\hline 293,839 & 263,518 & $1,946,234$ & 233,022 & MINNESOTA \\
\hline 136,655 & UA/NA & 122,903 & 175,803 & MISSOURI \\
\hline 161,787 & 0 & 481,974 & 149,845 & MONTREAL \\
\hline 140,394 & 448,584 & 361,614 & 69,247 & NEBRASKA \\
\hline 295,564 & 0 & 345,927 & 260,540 & NEW MEXICO \\
\hline UA/NA & UA/NA & 684,140 & 188,872 & NEW YORK \\
\hline 403,673 & UA/NA & 972,600 & 291,585 & NORTH CAROLINA \\
\hline 177,285 & 0 & 688,627 & 133,600 & NORTH CAROLINA STATE \\
\hline 202,761 & UA/NA & 481,567 & 105,541 & NORTHWESTERN \\
\hline 256,292 & 160,449 & 833,486 & 155,790 & NOTRE DAME \\
\hline 0 & 475,763 & 513,518 & 3,211 & $\mathrm{OHIO}$ \\
\hline 174,078 & $1,103,259$ & 364,798 & 92,365 & OHIO STATE \\
\hline 393,056 & 62,974 & 287,153 & 83,585 & OKLAHOMA \\
\hline UA/NA & UA/NA & UA/NA & 70,843 & OKLAHOMA STATE \\
\hline 157,824 & 34,528 & 587,625 & 170,829 & OREGON \\
\hline 552,404 & UA/NA & 595,987 & 179,564 & PENNSYLVANIA \\
\hline 324,404 & 9,101 & $1,730,892$ & 72,543 & PENNSYLVANIA STATE \\
\hline 430,659 & UA/NA & 900,573 & 99,053 & PITTSBURGH \\
\hline 395,621 & 0 & $1,028,682$ & 222,459 & PRINCETON \\
\hline 167,575 & 164,800 & 563,256 & 324,908 & PURDUE \\
\hline 155,047 & 150,039 & 88,590 & 48,680 & QUEEN'S \\
\hline 191,374 & UA/NA & 265,052 & 28,883 & RICE \\
\hline 160,825 & UA/NA & 648,770 & 75,154 & ROCHESTER \\
\hline
\end{tabular}

Hardware Document Delivery/

And Software Interlibrary Loan

(24) (25)

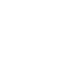

\section{.}

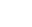




\section{ARL LIBRARY DATA TABLES, 2005-06 EXPENDITURES FOR ELECTRONIC RESOURCES}

\begin{tabular}{|c|c|c|c|c|c|c|}
\hline $\begin{array}{l}\text { (Survey Question \#) } \\
\text { INSTITUTION }\end{array}$ & Notes & $\begin{array}{r}\text { Computer } \\
\text { Files } \\
\\
(21)\end{array}$ & $\begin{array}{r}\text { Electronic } \\
\text { Serials } \\
(22)\end{array}$ & $\begin{array}{r}\text { Total } \\
\text { E-Materials } \\
(21+22)\end{array}$ & $\begin{array}{r}\text { Total } \\
\text { Materials } \\
\\
\text { (15) }\end{array}$ & $\begin{array}{l}\text { E-Materials As \% } \\
\text { Of Lib. Materials }\end{array}$ \\
\hline RUTGERS & GL+ & 2,540 & $3,731,127$ & $3,733,667$ & $10,610,826$ & 35.19 \\
\hline SASKATCHEWAN & GLM+ + & 16,713 & $3,927,205$ & $3,943,918$ & $7,763,830$ & 50.80 \\
\hline SOUTH CAROLINA & BLM & 19,644 & $3,660,313$ & $3,679,957$ & $7,066,564$ & 52.08 \\
\hline SOUTHERN CALIFORNIA & GLM+ & UA/NA & $3,359,071$ & $3,359,071$ & $12,962,417$ & 25.91 \\
\hline SOUTHERN ILLINOIS & $\mathrm{LM}+$ & 157,705 & $2,763,762$ & $2,921,467$ & $7,117,514$ & 41.05 \\
\hline SUNY-ALBANY & $\mathrm{G}^{+}$ & 7,591 & $1,663,616$ & $1,671,207$ & $5,231,629$ & 31.94 \\
\hline SUNY-BUFFALO & GLM+ + & 132,117 & $4,545,127$ & $4,677,244$ & $7,765,537$ & 60.23 \\
\hline SUNY-STONY BROOK & $\mathrm{BM}+$ & 3,522 & $2,124,891$ & $2,128,413$ & $6,590,728$ & 32.29 \\
\hline SYRACUSE & GL+ & 183,830 & $2,715,584$ & $2,899,414$ & $6,300,160$ & 46.02 \\
\hline TEMPLE & BLM+ & $1,395,622$ & $3,726,360$ & $5,121,982$ & $8,747,157$ & 58.56 \\
\hline TENNESSEE & GLM+ & 518,201 & $1,837,718$ & $2,355,919$ & $11,547,387$ & 20.40 \\
\hline TEXAS & $\mathrm{L}+^{+}$ & $1,778,728$ & $4,259,816$ & $6,038,544$ & $16,035,009$ & 37.66 \\
\hline TEXAS A\&M & $\mathrm{G}^{+}$ & 620,136 & $6,573,530$ & $7,193,666$ & $14,099,481$ & 51.02 \\
\hline TEXAS TECH & BLM+ & 13,185 & $2,001,951$ & $2,015,136$ & $9,503,529$ & 21.20 \\
\hline TORONTO & GL+ & 818,844 & $3,111,407$ & $3,930,250$ & $21,450,043$ & 18.32 \\
\hline TULANE & $\mathrm{LM}+$ & 802,648 & $2,769,655$ & $3,572,303$ & $7,675,475$ & 46.54 \\
\hline UTAH & BGLM+ + & UA/NA & $1,193,845$ & $1,193,845$ & $7,489,866$ & 15.94 \\
\hline VANDERBILT & GLM+ & 792,073 & $3,936,452$ & $4,728,525$ & $10,577,813$ & 44.70 \\
\hline VIRGINIA & LM+ & 562,541 & $3,594,792$ & $4,157,333$ & $10,039,183$ & 41.41 \\
\hline VIRGINIA TECH & $\mathrm{G}^{+}$ & 2,202 & $4,192,938$ & $4,195,140$ & $7,371,492$ & 56.91 \\
\hline WASHINGTON & $\mathrm{LM}+$ & 122,171 & $3,899,881$ & $4,022,052$ & $12,542,511$ & 32.07 \\
\hline WASHINGTON STATE & $\mathrm{G}^{+}$ & 109,043 & $2,911,670$ & $3,020,713$ & $5,628,649$ & 53.67 \\
\hline WASHINGTON U.-ST. LOUIS & GLM+ & 454,447 & $4,818,704$ & $5,273,151$ & $10,646,463$ & 49.53 \\
\hline WATERLOO & $\mathrm{G}^{+}$ & 16,419 & $3,506,766$ & $3,523,184$ & $6,180,735$ & 57.00 \\
\hline WAYNE STATE & GLM+ & 173,391 & $5,495,685$ & $5,669,076$ & $7,811,606$ & 72.57 \\
\hline WESTERN ONTARIO & GL+ & 59,095 & $5,434,891$ & $5,493,986$ & $9,174,734$ & 59.88 \\
\hline WISCONSIN & GLM+ & 770,352 & $2,836,208$ & $3,606,560$ & $11,582,710$ & 31.14 \\
\hline YALE & GLM+ & 455,285 & $4,234,069$ & $4,689,354$ & $33,913,555$ & 13.83 \\
\hline YORK & $\mathrm{G}^{+}$ & 431,791 & $4,093,751$ & $4,525,542$ & $8,384,544$ & 53.97 \\
\hline BOSTON PUBLIC & $\mathrm{G}^{+}$ & 250,503 & 816,928 & $1,067,431$ & $6,352,208$ & 16.80 \\
\hline CISTI & $\mathrm{G}^{+}$ & $1,301,992$ & $1,664,786$ & $2,966,778$ & $11,630,136$ & 25.51 \\
\hline CTR FOR RESEARCH LIBS & + & 3,164 & $\mathrm{UA} / \mathrm{NA}$ & 3,164 & 899,533 & 0.35 \\
\hline LIBRARY OF CONGRESS & BG+ + & 379,586 & $1,542,216$ & $1,921,802$ & $13,081,132$ & 14.69 \\
\hline NATL AGRICULTURAL LIB & $\mathrm{G}^{+}$ & 578,949 & $3,228,003$ & $3,806,952$ & $5,162,302$ & 73.75 \\
\hline LIB \& ARCHIVES CANADA & + & UA/NA & UA/NA & $\mathrm{UA} / \mathrm{NA}$ & $1,616,841$ & $\mathrm{UA} / \mathrm{NA}$ \\
\hline NATL LIB OF MEDICINE & $\mathrm{G}^{+}$ & 2,279 & 190,307 & 192,586 & $8,629,812$ & 2.23 \\
\hline NEW YORK PUBLIC & G & 0 & $1,861,239$ & $1,861,239$ & $13,705,178$ & 13.58 \\
\hline NEW YORK STATE & + & 0 & 29,113 & 29,113 & $3,664,047$ & 0.79 \\
\hline SMITHSONIAN & + & 0 & 195,993 & 195,993 & $2,063,349$ & 9.50 \\
\hline
\end{tabular}

UA/NA - Unavailable or Not Applicable

L - Includes Law library

M- Includes Medical library
+ - See Footnotes

B - Basis of volume count is bibliographic

G - Government documents not included in serials count 


\section{ARL LibRARY DATA TABLES, 2005-06 EXPENDITURES FOR ELECTRONIC RESOURCES}

Bibl. Utilities, Networks, and Consortia

Library

(23.a)

External

(23.b)

\begin{tabular}{|c|c|c|c|c|}
\hline 234,009 & UA/NA & 493,842 & 76,866 & RUTGERS \\
\hline 58,609 & 0 & 503,747 & 62,374 & SASKATCHEWAN \\
\hline 101,650 & 5,000 & 860,036 & 106,777 & SOUTH CAROLINA \\
\hline $1,695,449$ & 0 & $1,295,679$ & 107,319 & SOUTHERN CALIFORNIA \\
\hline 185,016 & 42,551 & 200,971 & 118,138 & SOUTHERN ILLINOIS \\
\hline 0 & 46,604 & 126,011 & 28,166 & SUNY-ALBANY \\
\hline UA/NA & UA/NA & UA/NA & UA/NA & SUNY-BUFFALO \\
\hline 289,790 & 344,319 & 411,374 & 46,133 & SUNY-STONY BROOK \\
\hline UA/NA & UA/NA & UA/NA & 117,710 & SYRACUSE \\
\hline 188,106 & 9,000 & 562,849 & 35,190 & TEMPLE \\
\hline 28,027 & 46,223 & 555,208 & 177,411 & TENNESSEE \\
\hline 202,658 & 37 & $2,590,351$ & 272,853 & TEXAS \\
\hline 0 & 497,865 & $1,085,615$ & 544,428 & TEXAS A\&M \\
\hline 343,583 & 54,750 & $1,407,056$ & 91,658 & TEXAS TECH \\
\hline $1,133,193$ & UA/NA & $2,309,521$ & 37,591 & TORONTO \\
\hline 166,467 & 85,088 & 123,430 & 23,550 & TULANE \\
\hline 238,151 & $1,528,982$ & $1,231,888$ & 50,444 & UTAH \\
\hline 234,601 & 0 & 496,837 & 137,912 & VANDERBILT \\
\hline 186,457 & 738,595 & $1,152,755$ & 139,884 & VIRGINIA \\
\hline 101,225 & 0 & 421,092 & 230,340 & VIRGINIA TECH \\
\hline 346,375 & 102,500 & $\mathrm{UA} / \mathrm{NA}$ & UA/NA & WASHINGTON \\
\hline 205,138 & 57,500 & 196,320 & 8,327 & WASHINGTON STATE \\
\hline 270,978 & 9,560 & $2,115,336$ & 83,314 & WASHINGTON U.-ST. LOUIS \\
\hline 0 & 0 & 234,373 & 79,273 & WATERLOO \\
\hline 90,377 & 0 & 813,781 & 63,322 & WAYNE STATE \\
\hline 108,579 & 0 & 322,271 & 0 & WESTERN ONTARIO \\
\hline 486,837 & 411,857 & $1,976,403$ & 381,118 & WISCONSIN \\
\hline 550,369 & 0 & 871,487 & 173,736 & YALE \\
\hline 61,065 & 0 & 203,138 & 8,795 & YORK \\
\hline 363,724 & 153,566 & 426,932 & 9,004 & BOSTON PUBLIC \\
\hline 8,955 & UA/NA & $2,266,969$ & UA/NA & CISTI \\
\hline 310,176 & 0 & 72,999 & 140,600 & CTR FOR RESEARCH LIBS \\
\hline 919,969 & 0 & $16,225,003$ & 0 & LIBRARY OF CONGRESS \\
\hline 0 & 578,736 & 559,844 & $1,020,016$ & NATL AGRICULTURAL LIB \\
\hline UA/NA & UA/NA & UA/NA & 73,459 & LIB \& ARCHIVES CANADA \\
\hline UA/NA & UA/NA & 285,239 & $1,626,334$ & NATL LIB OF MEDICINE \\
\hline 63,900 & 0 & 622,063 & 261,820 & NEW YORK PUBLIC \\
\hline UA/NA & 82,935 & 250,000 & UA/NA & NEW YORK STATE \\
\hline 133,593 & 0 & 0 & 4,321 & SMITHSONIAN \\
\hline
\end{tabular}

\author{
Hardware \\ And Software \\ Document Delivery/ \\ Interlibrary Loan
}

(24)
(25)
(Survey Question \#) INSTITUTION

RUTGERS

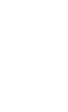




\section{ARL LIBRARY DATA TABLES, 2005-06 \\ SUMMARY DATA: EXPENDITURES FOR ELECTRONIC RESOURCES}

\begin{tabular}{|c|c|c|c|c|c|}
\hline (Survey Question \#) & $\begin{array}{r}\text { Computer } \\
\text { Files } \\
(21)\end{array}$ & $\begin{array}{r}\text { Electronic } \\
\text { Serials } \\
(22) \\
\end{array}$ & $\begin{array}{r}\text { Total } \\
\text { E-Materials } \\
\\
(21+22) \\
\end{array}$ & $\begin{array}{r}\text { Total } \\
\text { Materials } \\
\\
(15) \\
\end{array}$ & $\begin{array}{l}\text { E-Materials As \% } \\
\text { Of Lib. Materials }\end{array}$ \\
\hline \multicolumn{6}{|l|}{$\begin{array}{l}\text { UNIVERSITY } \\
\text { LIBRARIES: }\end{array}$} \\
\hline Median & 336,338 & $3,349,709$ & $3,792,873$ & $9,156,974$ & 43.14 \\
\hline High & $5,417,996$ & $8,158,340$ & $9,823,592$ & $33,913,555$ & 72.57 \\
\hline Low & 0 & $1,070,931$ & $1,070,931$ & $3,411,656$ & 13.83 \\
\hline Totals & $48,793,981$ & $383,127,163$ & $431,921,144$ & $1,159,553,716$ & \\
\hline $\begin{array}{l}\text { Number of } \\
\text { Libraries Responding }\end{array}$ & 102 & 108 & 109 & 113 & 109 \\
\hline
\end{tabular}

NONUNIVERSITY LIBRARIES:

\author{
Median \\ Totals \\ Number of \\ Libraries Responding
}

3,164
$2,516,473$
9

9

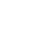

$1,179,572$

$9,528,585$

8

8

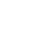

$\begin{array}{rr}1,067,431 & 5,757,255 \\ 12,045,058 & 66,804,538 \\ 9 & 10\end{array}$

13.58

9
GRAND TOTALS
$51,310,454$

$392,655,748$
$443,966,202$

$1,226,358,254$ 


\section{ARL LibRARY DATA TABLES, 2005-06 \\ SUMMARY DATA: EXPENDITURES FOR ELECTRONIC RESOURCES}

Bibl. Utilities, Networks, and Consortia Library

(23.a)

$2,111,812$

0

$26,000,437$

102

$1,800,317$

7
External

(23.b)

9,101

$1,894,324$

0

$15,930,476$

83

83
Hardware And Software

(24)
Document Delivery/

Interlibrary Loan

(Survey Question \#)

UNIVERSITY

LIBRARIES:

$\begin{array}{rr}503,828 & 106,777 \\ 2,678,825 & 544,428 \\ 61,839 & 0\end{array}$

$13,411,255$

Median

High

Low

$68,656,974$

Totals

104

107

Number of

Libraries Responding

NONUNIVERSITY LIBRARIES:

107,030

Median

$20,709,049$

$3,135,554$

Totals

815,237

9

8

Number of Libraries Responding

$27,800,754$

$16,745,713$

$89,366,023$

$16,546,810$

GRAND TOTALS 


\section{ARL LIBRARY DATA TABLES, 2005-06 Personnel and Public Services}

\begin{tabular}{|c|c|c|c|c|c|c|c|}
\hline $\begin{array}{l}\text { (Survey Question \#) } \\
\text { INSTITUTION }\end{array}$ & Notes & $\begin{array}{r}\text { Professional } \\
\text { Staff (FTE) } \\
\\
(26 . \mathrm{a})\end{array}$ & $\begin{array}{r}\text { Support } \\
\text { Staff (FTE) } \\
(26 . b)\end{array}$ & $\begin{array}{r}\text { Student } \\
\text { Assistants (FTE) } \\
(26 . c)\end{array}$ & $\begin{array}{r}\text { Total } \\
\text { Staff (FTE) } \\
\\
(26)\end{array}$ & $\begin{array}{r}\text { Staffed } \\
\text { Service Points } \\
(27)\end{array}$ & $\begin{array}{r}\text { Service Hours } \\
\text { Per Week } \\
(28)\end{array}$ \\
\hline ALABAMA & BGLM+ & 63 & 82 & 62 & 207 & 22 & 110 \\
\hline ALBERTA & GL+ & 82 & 215 & 27 & 324 & 22 & 103 \\
\hline ARIZONA & BGLM+ & 98 & 153 & 62 & 313 & 21 & 168 \\
\hline ARIZONA STATE & GL+ & 88 & 194 & 40 & 322 & 20 & 149 \\
\hline AUBURN & BG + & 51 & 55 & 34 & 140 & 11 & 111 \\
\hline BOSTON & GLM+ & 98 & 100 & 99 & 297 & 33 & 108 \\
\hline BOSTON COLLEGE & GL+ & 73 & 77 & 52 & 202 & 14 & 110 \\
\hline BRIGHAM YOUNG & BGL+ & 120 & 61 & 199 & 380 & 20 & 101 \\
\hline BRITISH COLUMBIA & GLM+ + & 112 & 199 & 38 & 349 & 33 & 101 \\
\hline BROWN & $\mathrm{M}+$ & 71 & 105 & 32 & 208 & 10 & 154 \\
\hline CALIFORNIA, BERKELEY & $\mathrm{L}+$ & 219 & 202 & 159 & 580 & 40 & 65 \\
\hline CALIFORNIA, DAVIS & GLM+ & 65 & 154 & 54 & 273 & 17 & 95 \\
\hline CALIFORNIA, IRVINE & BGM+ & 66 & 128 & 49 & 243 & 16 & 128 \\
\hline CALIFORNIA, LOS ANGELES & $\mathrm{LM}+$ & 174 & 297 & 151 & 622 & 30 & 97 \\
\hline CALIFORNIA, RIVERSIDE & BG & 43 & 96 & 33 & 172 & 15 & 91 \\
\hline CALIFORNIA, SAN DIEGO & $\mathrm{M}+$ & 87 & 183 & 67 & 337 & 18 & 168 \\
\hline CALIFORNIA, SANTA BARBAR & & 48 & 138 & 48 & 234 & 10 & 103 \\
\hline CASE WESTERN RESERVE & GLM+ & 62 & 53 & 32 & 147 & 17 & 97 \\
\hline CHICAGO & BGLM+ & 66 & 193 & 59 & 318 & 31 & 144 \\
\hline CINCINNATI & GLM+ & 105 & 70 & 53 & 228 & 24 & 95 \\
\hline COLORADO & BG+ + & 52 & 111 & 50 & 213 & 24 & 104 \\
\hline COLORADO STATE & G+ & 49 & 51 & 23 & 123 & 6 & 108 \\
\hline COLUMBIA & $\mathrm{LM}+$ & 311 & 247 & 140 & 698 & 50 & 107 \\
\hline CONNECTICUT & GLM+ & 88 & 75 & 55 & 218 & 22 & 114 \\
\hline CORNELL & GL+ & 118 & 320 & 115 & 553 & 52 & 144 \\
\hline DARTMOUTH & $\mathrm{GM}+$ & 61 & 117 & 28 & 206 & 18 & 114 \\
\hline DELAWARE & $\mathrm{G}^{+}$ & 60 & 110 & 37 & 207 & 16 & 100 \\
\hline DUKE & BLM+ & 173 & 156 & 32 & 361 & 21 & 137 \\
\hline EMORY & $\mathrm{LM}+$ & 103 & 148 & 44 & 295 & 29 & 98 \\
\hline FLORIDA & $\mathrm{LM}+$ & 109 & 202 & 128 & 439 & 20 & 104 \\
\hline FLORIDA STATE & BLM+ & 70 & 126 & 50 & 246 & 22 & 118 \\
\hline GEORGE WASHINGTON & BLM+ & 75 & 130 & 59 & 264 & 26 & 111 \\
\hline GEORGETOWN & BGLM+ + & 89 & 129 & 51 & 269 & 19 & 144 \\
\hline GEORGIA & $\mathrm{GL}^{+}$ & 71 & 185 & 38 & 294 & 20 & 110 \\
\hline GEORGIA TECH & G+ & 48 & 71 & 7 & 126 & 8 & 135 \\
\hline GUELPH & $\mathrm{G}^{+}$ & 40 & 72 & 19 & 131 & 13 & 133 \\
\hline HARVARD & GLM+ & 554 & 580 & 132 & 1,266 & UA/NA & $\mathrm{UA} / \mathrm{NA}$ \\
\hline HAWAII & $\mathrm{L}^{+}$ & 75 & 93 & 52 & 220 & 16 & 93 \\
\hline HOUSTON & BGL+ & 62 & 93 & 46 & 201 & 8 & 108 \\
\hline HOWARD & BLM+ & 52 & 71 & 48 & 171 & 19 & 126 \\
\hline ILLINOIS, CHICAGO & $\mathrm{GM}^{+}$ & 80 & 115 & 31 & 226 & 27 & 93 \\
\hline ILLINOIS, URBANA & GL+ & 185 & 197 & 121 & 503 & 50 & 119 \\
\hline
\end{tabular}

UA/NA - Unavailable or Not Applicable

L - Includes Law library

M- Includes Medical library G - Government documents n
* Figures in italics are derived from a sampling method rather than an actual annual count.
+ - See Footnotes

B - Basis of volume count is bibliographic

G - Government documents not included in serials count 


\section{ARL LibRARY DATA TABLES, 2005-06 Personnel and Public Services}

\begin{tabular}{|c|c|c|c|c|c|c|c|}
\hline \multicolumn{2}{|c|}{ Presentations } & \multirow{2}{*}{$\begin{array}{l}\text { Reference } \\
\text { Transactions* }\end{array}$} & \multirow{2}{*}{$\begin{array}{r}\text { Initial } \\
\text { Circulation }\end{array}$} & \multirow{2}{*}{$\begin{array}{r}\text { Total } \\
\text { Circulation }\end{array}$} & \multicolumn{2}{|c|}{ ILL/DD } & \multirow[b]{3}{*}{$\begin{array}{r}\text { (Survey Question \#) } \\
\text { INSTITUTION }\end{array}$} \\
\hline Groups* & Participants* & & & & Loaned & Borrowed & \\
\hline$(29)$ & (30) & (31) & (32) & (33) & (34) & (35) & \\
\hline 582 & 11,821 & 84,150 & 177,063 & 225,404 & 10,482 & 10,986 & ALABAMA \\
\hline 868 & 19,567 & 116,716 & UA/NA & 626,549 & 80,302 & 37,333 & ALBERTA \\
\hline 2,053 & 34,335 & 51,802 & 343,896 & 578,082 & 61,151 & 27,725 & ARIZONA \\
\hline 994 & 24,391 & 128,251 & 348,727 & $1,107,316$ & 56,361 & 29,649 & ARIZONA STATE \\
\hline 707 & 15,622 & 88,244 & 175,053 & 241,979 & 13,113 & 9,391 & AUBURN \\
\hline 793 & 12,389 & 83,509 & 278,770 & 373,737 & 16,230 & 14,546 & BOSTON \\
\hline 867 & 18,692 & 35,985 & 202,405 & 328,624 & 18,867 & 16,094 & BOSTON COLLEGE \\
\hline 2,065 & 30,630 & 191,807 & 574,056 & 751,011 & 36,103 & 38,989 & BRIGHAM YOUNG \\
\hline 1,462 & 26,204 & 128,854 & 751,932 & $3,247,381$ & 26,402 & 24,371 & BRITISH COLUMBIA \\
\hline 883 & 4,345 & 24,224 & 218,251 & 325,722 & 36,904 & 37,673 & BROWN \\
\hline 2,723 & 26,964 & 159,125 & 776,435 & UA/NA & 42,662 & 22,374 & CALIFORNIA, BERKELEY \\
\hline 631 & 12,301 & 79,332 & 279,961 & 459,333 & 28,305 & 29,978 & CALIFORNIA, DAVIS \\
\hline 583 & 17,165 & 67,697 & 176,189 & 393,406 & 28,501 & 29,287 & CALIFORNIA, IRVINE \\
\hline 1,319 & 22,264 & 100,732 & 547,815 & $2,147,178$ & 55,454 & 34,529 & CALIFORNIA, LOS ANGELES \\
\hline 464 & 10,397 & 30,232 & 171,170 & 234,915 & 22,618 & 17,511 & CALIFORNIA, RIVERSIDE \\
\hline 1,622 & 12,881 & 76,816 & 428,159 & 651,981 & 33,040 & 29,220 & CALIFORNIA, SAN DIEGO \\
\hline 745 & 10,815 & 147,110 & 240,836 & 414,482 & 23,432 & 32,628 & CALIFORNIA, SANTA BARBARA \\
\hline 540 & 7,767 & 23,754 & 144,260 & 218,209 & 81,645 & 42,151 & CASE WESTERN RESERVE \\
\hline 488 & 6,718 & 41,683 & 481,042 & 730,908 & 50,646 & 22,652 & CHICAGO \\
\hline 2,029 & 29,063 & 116,532 & 316,428 & 451,815 & 93,770 & 51,142 & CINCINNATI \\
\hline 682 & 13,368 & 124,339 & 394,737 & 669,019 & 48,208 & 31,708 & COLORADO \\
\hline 428 & 9,403 & 73,768 & 212,643 & 294,841 & 60,998 & 61,965 & COLORADO STATE \\
\hline 1,395 & 25,202 & 208,046 & 503,835 & $1,132,643$ & 50,096 & 50,297 & COLUMBIA \\
\hline 1,635 & 18,679 & 56,075 & 185,966 & 204,815 & 46,537 & 29,347 & CONNECTICUT \\
\hline 1,665 & 24,281 & 96,625 & 499,967 & $1,017,340$ & 61,490 & 34,587 & CORNELL \\
\hline UA/NA & UA/NA & UA/NA & 228,919 & 308,255 & 34,249 & 30,309 & DARTMOUTH \\
\hline 415 & 4,707 & 101,963 & 0 & 349,288 & 16,491 & 24,330 & DELAWARE \\
\hline 1,229 & 14,718 & 137,001 & 362,090 & 536,007 & 59,964 & 30,613 & DUKE \\
\hline 929 & 15,585 & 64,456 & 223,166 & 536,180 & 59,096 & 17,843 & EMORY \\
\hline 407 & 8,243 & 151,117 & 619,184 & 671,602 & 28,979 & 20,001 & FLORIDA \\
\hline 814 & 16,650 & 82,635 & 281,273 & 384,873 & 22,392 & 16,263 & FLORIDA STATE \\
\hline 1,006 & 18,642 & 47,200 & 189,318 & 303,030 & 34,591 & 56,506 & GEORGE WASHINGTON \\
\hline 518 & 12,299 & 84,252 & 247,927 & 384,976 & 38,388 & 24,320 & GEORGETOWN \\
\hline 837 & 14,423 & 99,315 & 308,325 & 463,636 & 49,233 & 8,698 & GEORGIA \\
\hline 224 & 4,054 & 11,777 & 81,521 & UA/NA & 9,852 & 8,634 & GEORGIA TECH \\
\hline 742 & 6,639 & 20,814 & 180,108 & 424,235 & 47,732 & 62,831 & GUELPH \\
\hline UA/NA & UA/NA & UA/NA & UA/NA & $1,958,747$ & 45,097 & 13,381 & HARVARD \\
\hline 232 & 4,214 & 75,413 & 274,863 & 483,314 & 4,394 & 17,676 & HAWAII \\
\hline 378 & 7,928 & 60,864 & 280,356 & 380,526 & 31,019 & 19,606 & HOUSTON \\
\hline 333 & 11,701 & 52,346 & 141,302 & 150,987 & 5,676 & 7,239 & HOWARD \\
\hline 632 & 9,364 & 35,795 & 173,657 & 489,180 & 76,941 & 36,930 & ILLINOIS, CHICAGO \\
\hline 1,052 & 15,715 & 273,738 & 537,666 & 618,262 & 83,999 & 87,264 & ILLINOIS, URBANA \\
\hline
\end{tabular}

UA/NA - Unavailable or Not Applicable

* Figures in italics are derived from a sampling method rather than an actual annual count. 


\section{ARL LIBRARY DATA TABLES, 2005-06 Personnel and Public Services}

\begin{tabular}{|c|c|c|c|c|c|c|c|}
\hline $\begin{array}{l}\text { (Survey Question \#) } \\
\text { INSTITUTION }\end{array}$ & Notes & $\begin{array}{r}\text { Professional } \\
\text { Staff (FTE) } \\
\\
(26 . \mathrm{a})\end{array}$ & $\begin{array}{r}\text { Support } \\
\text { Staff (FTE) } \\
(26 . b)\end{array}$ & $\begin{array}{r}\text { Student } \\
\text { Assistants (FTE) } \\
(26 . c)\end{array}$ & $\begin{array}{r}\text { Total } \\
\text { Staff (FTE) } \\
(26)\end{array}$ & $\begin{array}{r}\text { Staffed } \\
\text { Service Points } \\
\\
(27)\end{array}$ & $\begin{array}{r}\text { Service Hours } \\
\text { Per Week } \\
(28)\end{array}$ \\
\hline INDIANA & BL+ & 154 & 185 & 147 & 486 & 40 & 168 \\
\hline IOWA & GLM+ & 90 & 137 & 70 & 297 & 23 & 115 \\
\hline IOWA STATE & $\mathrm{G}^{+}$ & 51 & 93 & 38 & 182 & 12 & 105 \\
\hline JOHNS HOPKINS & BGM+ & 140 & 157 & 48 & 345 & 30 & 120 \\
\hline KANSAS & $\mathrm{LM}+$ & 103 & 110 & 76 & 289 & 11 & 140 \\
\hline KENT STATE & $\mathrm{G}^{+}$ & 54 & 38 & 192 & 284 & 13 & 99 \\
\hline KENTUCKY & GLM+ & 91 & 119 & 47 & 257 & 21 & 140 \\
\hline LAVAL & GLM+ & 60 & 154 & 12 & 226 & 17 & 88 \\
\hline LOUISIANA STATE & $\mathrm{LM}+$ & 54 & 96 & 53 & 203 & 16 & 100 \\
\hline LOUISVILLE & GLM+ & 51 & 90 & 44 & 185 & 20 & 98 \\
\hline MCGILL & GLM+ & 62 & 163 & 31 & 256 & 27 & 86 \\
\hline MCMASTER & GM & 34 & 107 & 24 & 165 & 13 & 100 \\
\hline MANITOBA & GLM+ & 61 & 127 & 30 & 218 & 33 & 100 \\
\hline MARYLAND & $\mathrm{G}^{+}$ & 145 & 97 & 213 & 455 & 8 & 140 \\
\hline MASSACHUSETTS & $\mathrm{G}^{+}$ & 55 & 76 & 46 & 177 & 17 & 148 \\
\hline MIT & $\mathrm{G}^{+}$ & 97 & 95 & 17 & 209 & 11 & 104 \\
\hline MIAMI & GLM+ & 82 & 125 & 50 & 257 & 19 & 120 \\
\hline MICHIGAN & $\mathrm{LM}+$ & 158 & 310 & 106 & 574 & $\mathrm{UA} / \mathrm{NA}$ & $\mathrm{UA} / \mathrm{NA}$ \\
\hline MICHIGAN STATE & $\mathrm{BGL}+$ & 79 & 124 & 89 & 292 & 23 & 148 \\
\hline MINNESOTA & BGLM+ & 106 & 214 & 88 & 408 & 52 & 100 \\
\hline MISSOURI & BGLM+ & 60 & 117 & 34 & 211 & 15 & 110 \\
\hline MONTREAL & GLM+ & 108 & 274 & 7 & 389 & 66 & 97 \\
\hline NEBRASKA & $\mathrm{GL}^{+}$ & 52 & 114 & 43 & 209 & 20 & 96 \\
\hline NEW MEXICO & $\mathrm{LM}+$ & 72 & 166 & 60 & 298 & 19 & 103 \\
\hline NEW YORK & GLM+ & 125 & 232 & 94 & 451 & 33 & 119 \\
\hline NORTH CAROLINA & GLM+ & 147 & 204 & 86 & 437 & 29 & 145 \\
\hline NORTH CAROLINA STATE & G+ & 117 & 106 & 53 & 276 & 17 & 146 \\
\hline NORTHWESTERN & GLM+ & 115 & 140 & 84 & 339 & 28 & 119 \\
\hline NOTRE DAME & GL+ & 67 & 158 & 36 & 261 & 19 & 126 \\
\hline OHIO & BGM+ & 64 & 68 & 47 & 179 & 18 & 146 \\
\hline OHIO STATE & $\mathrm{LM}+$ & 125 & 179 & 131 & 435 & 28 & 168 \\
\hline OKLAHOMA & GLM+ & 60 & 99 & 69 & 228 & 22 & 117 \\
\hline OKLAHOMA STATE & BGM & 62 & 80 & 70 & 212 & 20 & 119 \\
\hline OREGON & $\mathrm{GL}^{+}$ & 57 & 94 & 57 & 208 & 22 & 96 \\
\hline PENNSYLVANIA & $\mathrm{LM}+$ & 120 & 170 & 103 & 393 & 34 & 100 \\
\hline PENNSYLVANIA STATE & GLM+ & 148 & 388 & 76 & 612 & 63 & 168 \\
\hline PITTSBURGH & GLM+ & 120 & 172 & 70 & 362 & 50 & 118 \\
\hline PRINCETON & $\mathrm{B}+$ & 143 & 210 & 44 & 397 & 37 & 115 \\
\hline PURDUE & $\mathrm{G}^{+}$ & 66 & 126 & 59 & 251 & 21 & 119 \\
\hline QUEEN'S & BLM+ & 45 & 108 & 16 & 169 & 14 & 115 \\
\hline RICE & $\mathrm{G}^{+}$ & 59 & 61 & 12 & 132 & 12 & 143 \\
\hline ROCHESTER & BGM+ & 91 & 66 & 57 & 214 & 21 & 119 \\
\hline
\end{tabular}

UA/NA - Unavailable or Not Applicable

L - Includes Law library

M- Includes Medical library G - Government documents n
* Figures in italics are derived from a sampling method rather than an actual annual count.
+ - See Footnotes

B - Basis of volume count is bibliographic

G - Government documents not included in serials count 


\section{ARL LibRARY DATA TABLES, 2005-06 Personnel and Public Services}

\begin{tabular}{|c|c|c|c|c|c|c|c|}
\hline \multicolumn{2}{|c|}{ Presentations } & \multirow{2}{*}{$\begin{array}{l}\text { Reference } \\
\text { Transactions* }\end{array}$} & \multirow{2}{*}{$\begin{array}{r}\text { Initial } \\
\text { Circulation }\end{array}$} & \multirow{2}{*}{$\begin{array}{r}\text { Total } \\
\text { Circulation }\end{array}$} & \multicolumn{2}{|c|}{ ILL/DD } & \multirow[b]{3}{*}{$\begin{array}{r}\text { (Survey Question \#) } \\
\text { INSTITUTION }\end{array}$} \\
\hline Groups* & Participants* & & & & Loaned & Borrowed & \\
\hline (29) & (30) & (31) & (32) & (33) & (34) & (35) & \\
\hline 1,260 & 28,602 & 121,971 & 737,544 & 862,167 & 96,126 & 51,920 & INDIANA \\
\hline 805 & 18,504 & 85,621 & UA/NA & 585,231 & 63,356 & 28,761 & IOWA \\
\hline 292 & 9,378 & 27,703 & 232,950 & 266,821 & 33,827 & 14,450 & IOWA STATE \\
\hline 403 & 6,834 & 59,482 & 265,988 & 456,227 & 36,097 & 36,266 & JOHNS HOPKINS \\
\hline 1,081 & 19,089 & 79,832 & 422,469 & 551,853 & 58,466 & 42,771 & KANSAS \\
\hline 795 & 13,110 & 30,757 & 279,606 & 327,167 & 13,710 & 7,725 & KENT STATE \\
\hline 953 & 11,832 & 63,914 & 260,633 & 326,533 & 31,662 & 24,392 & KENTUCKY \\
\hline 520 & 8,100 & 142,026 & 518,732 & 627,177 & 20,088 & 12,940 & LAVAL \\
\hline 964 & 4,835 & 46,598 & 134,376 & 234,363 & 7,733 & 23,341 & LOUISIANA STATE \\
\hline 730 & 13,521 & 53,307 & 363,766 & 553,944 & 22,570 & 32,090 & LOUISVILLE \\
\hline 1,491 & 20,500 & 107,582 & 705,462 & $1,019,632$ & 17,375 & 20,656 & MCGILL \\
\hline 634 & 11,253 & 54,219 & 297,700 & 466,815 & 16,061 & 14,840 & MCMASTER \\
\hline 1,156 & 11,669 & 92,432 & 366,210 & 579,376 & 22,026 & 31,600 & MANITOBA \\
\hline 986 & 22,736 & 195,123 & 338,699 & 559,021 & 16,747 & 40,366 & MARYLAND \\
\hline 223 & 3,807 & 62,875 & 317,636 & 459,314 & 59,838 & 36,190 & MASSACHUSETTS \\
\hline 383 & 7,195 & 36,798 & 206,703 & 367,115 & 11,261 & 12,558 & MIT \\
\hline 1,482 & 17,654 & 94,508 & 194,423 & 266,141 & 41,279 & 16,110 & MIAMI \\
\hline UA/NA & UA/NA & UA/NA & UA/NA & UA/NA & UA/NA & UA/NA & MICHIGAN \\
\hline 828 & 29,697 & 45,211 & 314,935 & 766,347 & 47,915 & 35,221 & MICHIGAN STATE \\
\hline 1,452 & 23,251 & 145,151 & 412,430 & 761,544 & 177,020 & 36,326 & MINNESOTA \\
\hline 857 & 10,390 & 62,289 & 212,175 & 273,284 & 62,761 & 39,219 & MISSOURI \\
\hline 1,091 & 14,693 & 108,008 & 502,632 & 967,179 & 27,664 & 20,116 & MONTREAL \\
\hline 221 & 5,584 & 44,474 & 244,535 & 418,779 & 27,857 & 20,172 & NEBRASKA \\
\hline 1,340 & 19,937 & 58,828 & 222,786 & 324,003 & 23,540 & 22,995 & NEW MEXICO \\
\hline 1,073 & 16,756 & 133,794 & 483,479 & $1,043,893$ & 25,215 & 29,160 & NEW YORK \\
\hline 1,479 & 26,822 & 125,121 & UA/NA & 986,118 & 56,504 & 14,934 & NORTH CAROLINA \\
\hline 499 & 12,857 & 49,642 & 276,788 & 380,244 & 13,622 & 25,006 & NORTH CAROLINA STATE \\
\hline 657 & 7,717 & 85,194 & 236,022 & 365,468 & 43,569 & 30,510 & NORTHWESTERN \\
\hline 454 & 7,089 & 24,834 & 227,528 & 330,996 & 23,822 & 17,667 & NOTRE DAME \\
\hline 1,171 & 16,244 & 55,865 & 319,347 & 475,864 & 76,678 & 54,503 & $\mathrm{OHIO}$ \\
\hline 1,066 & 21,846 & UA/NA & 450,833 & $1,928,444$ & 108,828 & 93,669 & OHIO STATE \\
\hline 1,147 & 12,141 & 60,904 & 194,944 & 222,871 & 35,697 & 33,878 & OKLAHOMA \\
\hline 1,671 & 31,174 & 109,631 & 346,684 & 453,572 & 39,036 & 23,077 & OKLAHOMA STATE \\
\hline 589 & 10,935 & 66,667 & 253,676 & 317,232 & 69,623 & 63,238 & OREGON \\
\hline 1,036 & 17,091 & UA/NA & 419,524 & 559,661 & 64,483 & 79,534 & PENNSYLVANIA \\
\hline 2,682 & 44,429 & 214,324 & 701,682 & $1,046,822$ & 61,885 & 51,789 & PENNSYLVANIA STATE \\
\hline 1,988 & 24,882 & 185,356 & 428,056 & 477,593 & 68,125 & 23,914 & PITTSBURGH \\
\hline 695 & 8,570 & 28,519 & 357,653 & 438,133 & 34,402 & 33,579 & PRINCETON \\
\hline 255 & 7,372 & 54,252 & 172,474 & 604,455 & 31,666 & 28,274 & PURDUE \\
\hline 689 & 10,471 & 40,859 & 231,582 & 492,367 & 23,016 & 13,118 & QUEEN'S \\
\hline 165 & 2,369 & UA/NA & 123,157 & 215,057 & 13,802 & 10,758 & RICE \\
\hline 762 & 10,147 & 47,950 & 194,575 & 229,478 & 25,694 & 21,834 & ROCHESTER \\
\hline
\end{tabular}

UA/NA - Unavailable or Not Applicable

* Figures in italics are derived from a sampling method rather than an actual annual count. 


\section{ARL LIBRARY DATA TABLES, 2005-06 Personnel and Public Services}

\begin{tabular}{|c|c|c|c|c|c|c|c|}
\hline $\begin{array}{l}\text { (Survey Question \#) } \\
\text { INSTITUTION }\end{array}$ & Notes & $\begin{array}{r}\text { Professional } \\
\text { Staff (FTE) } \\
\\
(26 . \mathrm{a})\end{array}$ & $\begin{array}{r}\text { Support } \\
\text { Staff (FTE) } \\
(26 . b)\end{array}$ & $\begin{array}{r}\text { Student } \\
\text { Assistants (FTE) } \\
(26 . c)\end{array}$ & $\begin{array}{r}\text { Total } \\
\text { Staff (FTE) } \\
\\
(26)\end{array}$ & $\begin{array}{r}\text { Staffed } \\
\text { Service Points } \\
\\
(27)\end{array}$ & $\begin{array}{r}\text { Service Hours } \\
\text { Per Week } \\
(28)\end{array}$ \\
\hline RUTGERS & GL+ & 103 & 235 & 100 & 438 & 35 & 105 \\
\hline SASKATCHEWAN & GLM+ + & 47 & 94 & 11 & 152 & 13 & 90 \\
\hline SOUTH CAROLINA & BLM & 68 & 101 & 108 & 277 & 10 & 152 \\
\hline SOUTHERN CALIFORNIA & GLM+ & 113 & 131 & 131 & 375 & 29 & 159 \\
\hline SOUTHERN ILLINOIS & LM+ & 52 & 93 & 62 & 207 & 16 & 102 \\
\hline SUNY-ALBANY & $\mathrm{G}^{+}$ & 60 & 67 & 32 & 159 & 11 & 109 \\
\hline SUNY-BUFFALO & GLM+ & 134 & 47 & 68 & 249 & 18 & 141 \\
\hline SUNY-STONY BROOK & $\mathrm{BM}+$ & 95 & 25 & 39 & 159 & 11 & 106 \\
\hline SYRACUSE & GL+ & 67 & 134 & 27 & 228 & 22 & 104 \\
\hline TEMPLE & BLM+ + & 73 & 96 & 60 & 229 & 29 & 108 \\
\hline TENNESSEE & GLM+ & 95 & 140 & 49 & 284 & 29 & 168 \\
\hline TEXAS & $\mathrm{L}+$ & 143 & 286 & 107 & 536 & 29 & 107 \\
\hline TEXAS A\&M & $\mathrm{G}^{+}$ & 131 & 137 & 99 & 367 & 16 & 142 \\
\hline TEXAS TECH & BLM+ + & 101 & 117 & 88 & 306 & 29 & 130 \\
\hline TORONTO & $\mathrm{GL}^{+}$ & 187 & 352 & 149 & 688 & 107 & 113 \\
\hline TULANE & $\mathrm{LM}^{+}$ & 51 & 82 & 42 & 175 & 11 & 115 \\
\hline UTAH & BGLM+ & 76 & 190 & 90 & 356 & 20 & 116 \\
\hline VANDERBILT & GLM+ & 101 & 106 & 38 & 245 & 16 & 149 \\
\hline VIRGINIA & LM+ & 97 & 217 & 59 & 373 & 32 & 149 \\
\hline VIRGINIA TECH & $\mathrm{G}^{+}$ & 36 & 90 & 36 & 162 & 9 & 102 \\
\hline WASHINGTON & LM+ & 189 & 212 & 126 & 527 & 50 & 135 \\
\hline WASHINGTON STATE & $\mathrm{G}^{+}$ & 49 & 84 & 36 & 169 & 25 & 91 \\
\hline WASHINGTON U.-ST. LOUIS & GLM+ & 113 & 135 & 63 & 311 & 23 & 116 \\
\hline WATERLOO & $\mathrm{G}^{+}$ & 35 & 99 & 43 & 177 & 15 & 106 \\
\hline WAYNE STATE & GLM+ & 96 & 90 & 81 & 267 & 18 & 168 \\
\hline WESTERN ONTARIO & GL+ & 71 & 103 & 21 & 195 & 8 & 107 \\
\hline WISCONSIN & GLM+ & 233 & 172 & 172 & 577 & 54 & 148 \\
\hline YALE & GLM+ & 260 & 375 & 71 & 706 & 44 & 111 \\
\hline YORK & $\mathrm{G}^{+}$ & 66 & 127 & 59 & 252 & 20 & 93 \\
\hline BOSTON PUBLIC & $\mathrm{G}^{+}$ & 170 & 260 & $\mathrm{UA} / \mathrm{NA}$ & 430 & 96 & 68 \\
\hline CISTI & $\mathrm{G}^{+}$ & 168 & 160 & 4 & 332 & 1 & 40 \\
\hline CTR FOR RESEARCH LIBS & + & 25 & 16 & 26 & 67 & 2 & 35 \\
\hline LIBRARY OF CONGRESS & BG+ & 1,378 & 2,596 & 19 & 3,993 & 38 & 65 \\
\hline NATL AGRICULTURAL LIB & $\mathrm{G}^{+}$ & 133 & 58 & 1 & 192 & 13 & 45 \\
\hline LIB \& ARCHIVES CANADA & + & UA/NA & UA/NA & UA/NA & UA/NA & 8 & 35 \\
\hline NATL LIB OF MEDICINE & $\mathrm{G}^{+}$ & 187 & 71 & 16 & 274 & 5 & 48 \\
\hline NEW YORK PUBLIC & G & 281 & 281 & 102 & 664 & 42 & 46 \\
\hline NEW YORK STATE & + & 55 & 90 & 4 & 149 & 5 & 40 \\
\hline SMITHSONIAN & + & 60 & 68 & 2 & 130 & 31 & 168 \\
\hline
\end{tabular}

UA/NA - Unavailable or Not Applicable

L - Includes Law library

M- Includes Medical library G - Government documents n
* Figures in italics are derived from a sampling method rather than an actual annual count.
+ - See Footnotes

B - Basis of volume count is bibliographic

G - Government documents not included in serials count 


\section{ARL LibRARY DATA TABLES, 2005-06 Personnel and Public Services}

\begin{tabular}{|c|c|c|c|c|c|c|c|}
\hline \multicolumn{2}{|c|}{ Presentations } & \multirow{2}{*}{$\begin{array}{l}\text { Reference } \\
\text { Transactions* }\end{array}$} & \multirow{2}{*}{$\begin{array}{r}\text { Initial } \\
\text { Circulation }\end{array}$} & \multirow{2}{*}{$\begin{array}{r}\text { Total } \\
\text { Circulation }\end{array}$} & \multicolumn{2}{|c|}{ ILL/DD } & \multirow[b]{3}{*}{$\begin{array}{r}\text { (Survey Question \#) } \\
\text { INSTITUTION }\end{array}$} \\
\hline Groups* & Participants* & & & & Loaned & Borrowed & \\
\hline (29) & (30) & (31) & (32) & (33) & (34) & (35) & \\
\hline 1,071 & 24,294 & 103,545 & 301,597 & 599,533 & 34,055 & 27,412 & RUTGERS \\
\hline 491 & 6,728 & 26,900 & 270,513 & 375,015 & 23,758 & 21,119 & SASKATCHEWAN \\
\hline 760 & 15,754 & 174,420 & 396,211 & 553,188 & 21,813 & 18,794 & SOUTH CAROLINA \\
\hline 1,049 & 13,092 & 64,582 & 350,376 & 465,990 & 24,385 & 16,103 & SOUTHERN CALIFORNIA \\
\hline 969 & 8,528 & 54,706 & 234,563 & 355,080 & 57,968 & 36,342 & SOUTHERN ILLINOIS \\
\hline 735 & 6,615 & 73,406 & 90,715 & 198,965 & 13,921 & 18,285 & SUNY-ALBANY \\
\hline 753 & 19,109 & 50,835 & 312,688 & 361,629 & 44,521 & 17,064 & SUNY-BUFFALO \\
\hline 556 & 9,244 & 76,782 & 175,960 & 239,607 & 24,294 & 15,576 & SUNY-STONY BROOK \\
\hline 584 & 10,836 & 55,401 & 191,411 & 273,947 & 14,230 & 21,179 & SYRACUSE \\
\hline 535 & 12,903 & 60,861 & 193,219 & 295,225 & 22,832 & 19,391 & TEMPLE \\
\hline 858 & 16,123 & 90,368 & 286,335 & 359,568 & 36,035 & 29,382 & TENNESSEE \\
\hline 1,176 & 55,221 & 229,342 & $1,877,765$ & $2,771,993$ & 48,065 & 34,428 & TEXAS \\
\hline 441 & 10,181 & 45,016 & 738,117 & $1,363,291$ & 45,016 & 58,344 & TEXAS A\&M \\
\hline 1,172 & 18,597 & 73,372 & 202,560 & 309,974 & 33,632 & 26,959 & TEXAS TECH \\
\hline 2,498 & 32,414 & 575,055 & $1,386,399$ & $1,997,131$ & 35,439 & 10,748 & TORONTO \\
\hline 154 & 2,777 & 11,229 & 65,896 & 98,841 & 4,411 & 6,755 & TULANE \\
\hline 1,499 & 21,343 & 246,173 & 198,113 & 537,217 & 39,396 & 26,609 & UTAH \\
\hline 744 & 13,468 & 39,516 & 213,301 & 353,835 & 28,686 & 19,610 & VANDERBILT \\
\hline 1,243 & 14,016 & 122,794 & 624,914 & $1,028,028$ & 44,397 & 32,501 & VIRGINIA \\
\hline 823 & 16,608 & 24,457 & 234,610 & 276,095 & 21,978 & 22,079 & VIRGINIA TECH \\
\hline 1,440 & 30,023 & 139,416 & 736,744 & $1,481,321$ & 72,447 & 55,011 & WASHINGTON \\
\hline 779 & 13,009 & 49,628 & 286,702 & 491,524 & 46,040 & 18,426 & WASHINGTON STATE \\
\hline 906 & 5,859 & 81,599 & 268,438 & 502,798 & 48,775 & 48,880 & WASHINGTON U.-ST. LOUIS \\
\hline 166 & 5,166 & 27,703 & 228,928 & 589,089 & 68,736 & 46,413 & WATERLOO \\
\hline 617 & 13,166 & 52,583 & 131,461 & 200,120 & 38,802 & 30,880 & WAYNE STATE \\
\hline 842 & 16,710 & 43,951 & 474,136 & 774,931 & 19,825 & 6,721 & WESTERN ONTARIO \\
\hline 2,302 & 30,316 & UA/NA & 550,919 & 882,731 & 115,302 & 75,331 & WISCONSIN \\
\hline 1,294 & 10,770 & 95,547 & 245,113 & 610,077 & 49,320 & 38,237 & YALE \\
\hline 844 & 25,749 & 170,443 & 513,076 & $1,068,846$ & 14,832 & 4,926 & YORK \\
\hline 10,044 & 168,070 & $1,008,736$ & $2,601,520$ & $2,601,520$ & 20,045 & 11,871 & BOSTON PUBLIC \\
\hline 1,484 & 1,484 & 22,925 & 15,244 & 33,463 & 573,501 & 23,615 & CISTI \\
\hline 48 & 1,585 & UA/NA & 80,581 & 80,581 & 27,809 & 3 & CTR FOR RESEARCH LIBS \\
\hline 1,122 & 46,701 & $1,006,846$ & 117,835 & 117,835 & 24,065 & 9 & LIBRARY OF CONGRESS \\
\hline 258 & 42,908 & 12,940 & 4,095 & 5,159 & 18,354 & 4,447 & NATL AGRICULTURAL LIB \\
\hline UA/NA & UA/NA & UA/NA & UA/NA & UA/NA & UA/NA & UA/NA & LIB \& ARCHIVES CANADA \\
\hline 600 & 9,956 & 91,784 & 245,996 & UA/NA & 328,661 & 235 & NATL LIB OF MEDICINE \\
\hline 652 & 7,374 & 621,992 & UA/NA & UA/NA & 5,031 & 1,176 & NEW YORK PUBLIC \\
\hline 114 & 1,825 & 79,817 & 36,044 & 41,153 & 31,261 & 49,813 & NEW YORK STATE \\
\hline 108 & 4,114 & 84,963 & 36,414 & 252,667 & 7,363 & 5,822 & SMITHSONIAN \\
\hline
\end{tabular}

UA/NA - Unavailable or Not Applicable

* Figures in italics are derived from a sampling method rather than an actual annual count. 


\section{ARL LIBRARY DATA TABLES, 2005-06 \\ Summary Data: Personnel and Public Services}

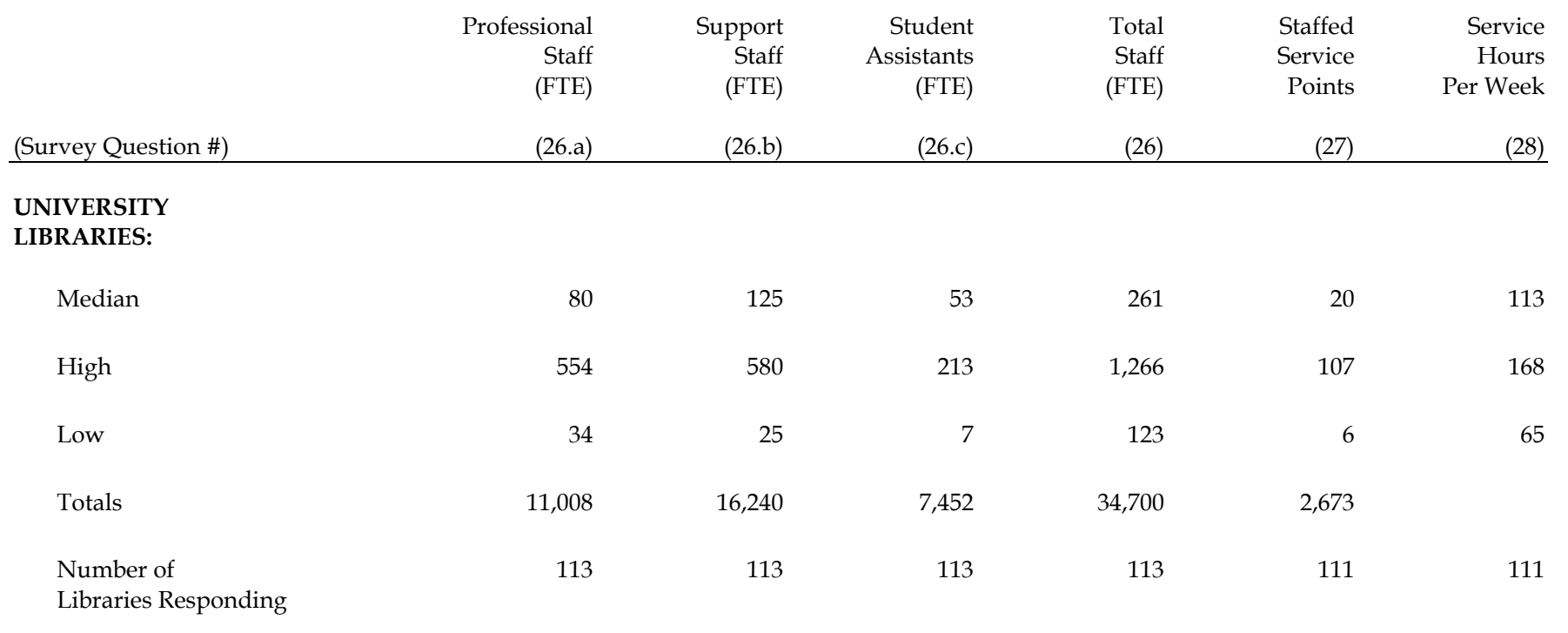

\section{NONUNIVERSITY LIBRARIES:}

Median
Totals
Number of
Libraries Responding

GRAND TOTALS

168
2,457

9
90

3,600

9

13,465
19,840

7,626

40,931

2,914 


\section{ARL LibRARY DATA TABLES, 2005-06 Summary Data: Personnel and Public Services}

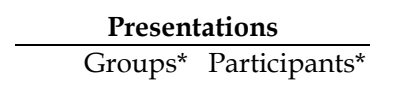

(29)

(30)

Reference

Transactions* Circulation

Total

Circulation

ILL/DD

(31)

(32)

(33)

(35)

(Survey Question \#)

UNIVERSITY

LIBRARIES:

$\begin{array}{rrrrrrr}833 & 13,267 & 73,587 & 279,188 & 464,813 & 35,568 & 27,186 \\ 2,723 & 55,221 & 575,055 & 1,877,765 & 3,247,381 & 177,020 & 93,669 \\ 154 & 2,369 & 11,229 & 0 & 98,841 & 4,394 & 4,926 \\ 103,297 & 1,709,848 & 9,595,345 & 37,310,445 & 67,273,678 & 4,574,967 & 3,321,549 \\ 110 & 110 & 106 & 108 & 110 & 112 & 112\end{array}$

Median

High

Low

Totals

Number of Libraries Responding

NONUNIVERSITY LIBRARIES:

$\begin{array}{rrrrrrr}600 & 7,374 & 88,374 & 58,498 & 80,581 & 24,065 & 4,447 \\ 14,430 & 284,017 & 2,930,003 & 3,137,729 & 3,132,378 & 1,036,090 & 96,991 \\ 9 & 9 & 8 & 8 & 7 & 9 & 9 \\ \text { Number of } \\ \text { Libraries Responding }\end{array}$

$\begin{array}{llllll}117,727 & 1,993,865 & 12,525,348 & 40,448,174 & 70,406,056 & 5,611,057\end{array}$

GRAND TOTALS 


\section{ANALYSIS OF SELECTED VARIABLES OF ARL UNIVERSITY LIBRARIES, 2005-06}

The percentages and ratios below are select indicators that describe the condition of ARL university libraries. The high and low figures indicate the range, while the mean and median indicate the central tendency of the distributions for ARL university libraries. Note that a distribution is normal when the mean and the median figures are identical. If the mean is larger than the median then the distribution is positively skewed, and if the opposite is true the distribution is negatively skewed.

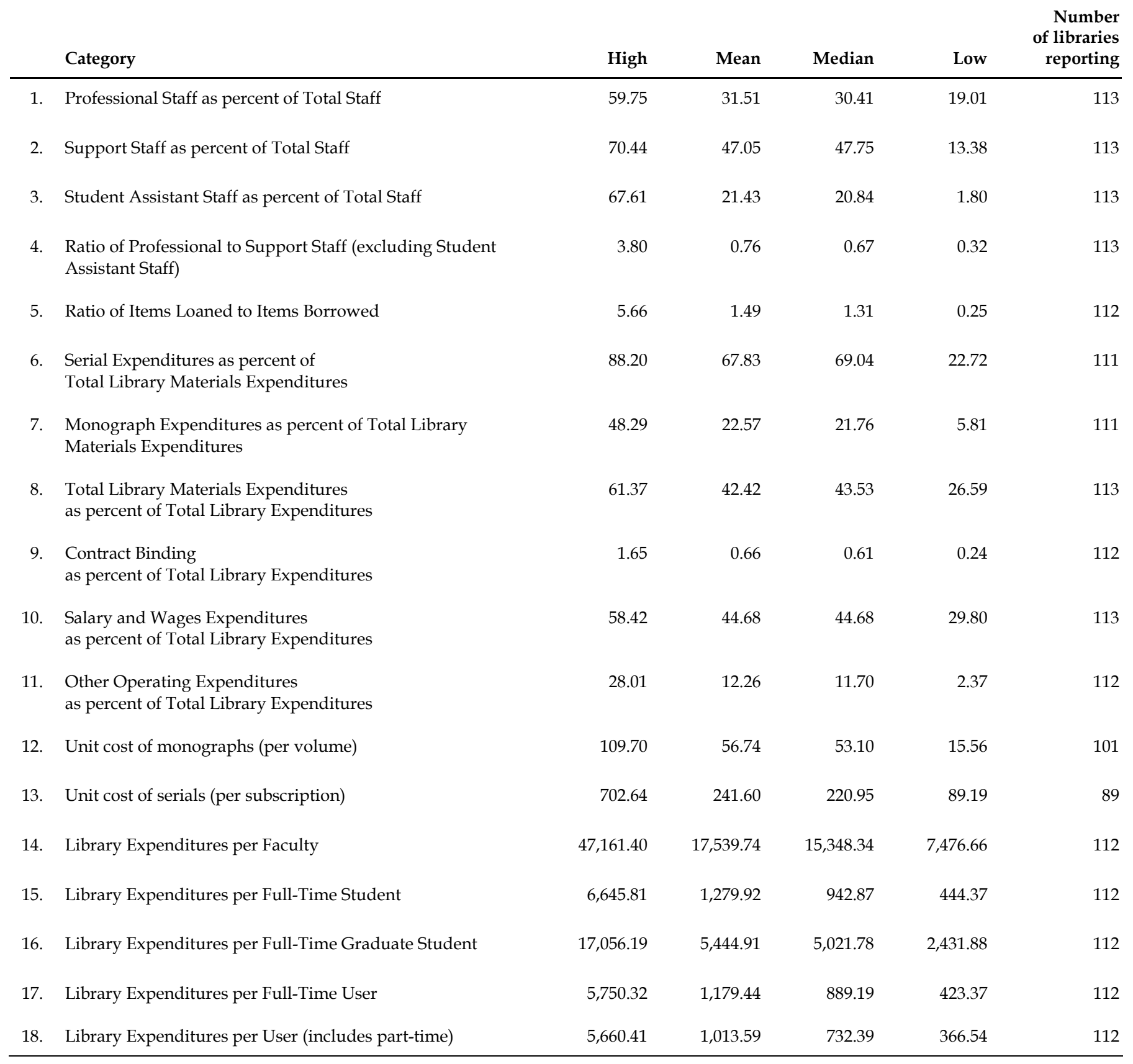




\section{Ph.D., Faculty, AND EnRollment Statistics *}

ENROLLMENTS

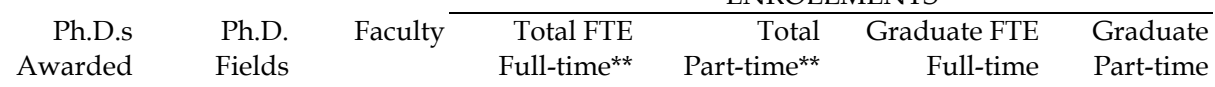

(Survey Question \#)

(36)

(37)

(38)

(39)

(40)

(41)

$(42)$ INSTITUTION

\begin{tabular}{|c|c|c|c|c|c|c|c|}
\hline ALABAMA & 154 & 44 & 846 & 17,987 & 3,250 & 2,155 & 1,532 \\
\hline ALBERTA & 297 & 116 & 1,503 & 31,501 & 3,888 & 4,356 & 1,717 \\
\hline ARIZONA & 388 & 85 & 1,557 & 30,776 & 6,260 & 6,051 & 2,523 \\
\hline ARIZONA STATE & 333 & 46 & 2,268 & 47,014 & 14,019 & 7,321 & 4,757 \\
\hline AUBURN & 152 & 38 & 1,176 & 20,035 & 3,298 & 1,466 & 1,703 \\
\hline BOSTON & 350 & 61 & 2,339 & 25,396 & 5,561 & 8,012 & 4,251 \\
\hline BOSTON COLLEGE & 114 & 36 & 662 & 11,895 & 2,675 & 2,462 & 2,274 \\
\hline BRIGHAM YOUNG & 75 & 25 & 1,282 & 29,400 & 4,667 & 1,940 & 1,329 \\
\hline BRITISH COLUMBIA & 325 & 89 & 2,303 & 32,344 & 14,712 & 6,718 & 1,460 \\
\hline BROWN & 209 & 45 & 635 & 7,899 & 962 & 1,968 & 117 \\
\hline CALIFORNIA, BERKELEY & 799 & 82 & 1,450 & 30,875 & 2,672 & 7,511 & 1,478 \\
\hline CALIFORNIA, DAVIS & 406 & 64 & 1,891 & 27,064 & 2,573 & 6,410 & 513 \\
\hline CALIFORNIA, IRVINE & 266 & 42 & 1,423 & 23,301 & 1,099 & 3,968 & 502 \\
\hline CALIFORNIA, LOS ANGELES & 657 & 79 & 2,505 & 37,211 & 1,188 & 10,814 & 160 \\
\hline CALIFORNIA, RIVERSIDE & 165 & 38 & 851 & 16,352 & 523 & 2,064 & 19 \\
\hline CALIFORNIA, SAN DIEGO & 353 & 68 & 1,717 & 24,733 & 1,102 & 5,155 & 103 \\
\hline CALIFORNIA, SANTA BARBARA & 235 & 44 & 975 & 20,356 & 660 & 2,924 & 15 \\
\hline CASE WESTERN RESERVE & 188 & 58 & 604 & 7,490 & 2,125 & 3,776 & 1,890 \\
\hline CHICAGO & 398 & 64 & 1,309 & 11,435 & 2,715 & 6,821 & 2,658 \\
\hline CINCINNATI & 264 & 66 & 1,224 & 25,139 & 10,105 & 5,054 & 3,366 \\
\hline COLORADO & 296 & 44 & 1,248 & 25,677 & 5,912 & 1,904 & 3,475 \\
\hline COLORADO STATE & 187 & 41 & 950 & 20,930 & 5,616 & 1,935 & 3,155 \\
\hline COLUMBIA & 523 & 108 & 1,928 & 22,757 & 6,642 & 14,026 & 5,698 \\
\hline CONNECTICUT & 307 & 69 & 1,596 & 22,624 & 5,459 & 4,554 & 3,004 \\
\hline CORNELL & 476 & 82 & 1,700 & 19,599 & 43 & 5,937 & 21 \\
\hline DARTMOUTH & 70 & 15 & 766 & 5,638 & 142 & 1,588 & 82 \\
\hline DELAWARE & 222 & 43 & 1,077 & 17,956 & 3,026 & 2,502 & 932 \\
\hline DUKE & 292 & 48 & 964 & 13,493 & 582 & 7,023 & 518 \\
\hline EMORY & 173 & 29 & 685 & 11,287 & 847 & 4,866 & 758 \\
\hline FLORIDA & 718 & 109 & 2,229 & 39,966 & 5,659 & 9,338 & 2,997 \\
\hline FLORIDA STATE & 325 & 74 & 1,265 & 32,774 & 6,878 & 5,237 & 2,689 \\
\hline GEORGE WASHINGTON & 95 & 28 & 1,014 & 15,579 & 8,520 & 5,838 & 7,500 \\
\hline GEORGETOWN & 84 & 23 & 1,166 & 12,231 & 1,421 & 5,727 & 1,206 \\
\hline GEORGIA & 374 & 84 & 1,725 & 29,354 & 4,605 & 6,058 & 2,464 \\
\hline GEORGIA TECH & 400 & 47 & 810 & 15,261 & 1,874 & 4,269 & 1,025 \\
\hline GUELPH & 91 & UA/NA & 790 & 16,748 & 1,416 & 1,824 & 144 \\
\hline HARVARD & 473 & 74 & 2,497 & 19,263 & 5,754 & 12,166 & 3,126 \\
\hline HAWAII & 147 & 50 & 1,281 & 14,744 & 2,148 & 3,538 & 1,004 \\
\hline HOUSTON & 241 & 33 & 1,218 & 24,088 & 10,246 & 4,486 & 2,448 \\
\hline HOWARD & 93 & 29 & 1,105 & 9,834 & 1,096 & 1,756 & 631 \\
\hline ILLINOIS, CHICAGO & 258 & 57 & 1,163 & 17,998 & 4,556 & 4,265 & 3,139 \\
\hline ILLINOIS, URBANA & 661 & 84 & 2,176 & 38,631 & 3,307 & 9,389 & 2,310 \\
\hline INDIANA & 389 & 90 & 1,865 & 33,707 & 4,540 & 5,469 & 2,950 \\
\hline
\end{tabular}

* - Figures were reported on the ARL Questionnaire and have not been verified with the U.S. NCES IPEDS survey ** - Includes both undergraduate and graduate students

UA/NA - Unavailable or Not Applicable 


\section{Ph.D., Faculty, AND EnRollment Statistics *}

ENROLLMENTS

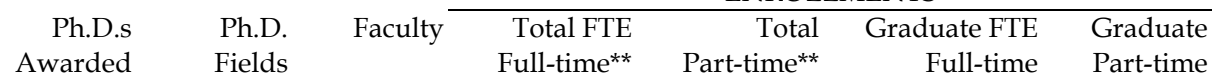

(Survey Question \#)

(36)

(37)

(38)

(39)

(40)

(41)

$(42)$

INSTITUTION

\begin{tabular}{|c|c|c|c|c|c|c|c|}
\hline IOWA & 316 & 61 & 1,132 & 23,008 & 5,418 & 4,814 & 3,312 \\
\hline IOWA STATE & 281 & 83 & 1,419 & 22,465 & 3,276 & 3,032 & 1,977 \\
\hline JOHNS HOPKINS & 388 & 57 & 1,342 & 10,795 & 8,430 & 5,183 & 7,947 \\
\hline KANSAS & 239 & 72 & 1,649 & 23,008 & 5,941 & 4,120 & 3,438 \\
\hline KENT STATE & 249 & 67 & 856 & 18,719 & 4,903 & 2,389 & 2,488 \\
\hline KENTUCKY & 276 & 60 & 1,211 & 21,485 & 4,187 & 4,435 & 2,535 \\
\hline LAVAL & 272 & 64 & 1,443 & 25,449 & 18,571 & 5,462 & 4,886 \\
\hline LOUISIANA STATE & 254 & 55 & 1,436 & 28,951 & 3,403 & 3,821 & 1,411 \\
\hline LOUISVILLE & 445 & 30 & 1,781 & 16,246 & 6,117 & 6,703 & 2,540 \\
\hline MCGILL & 222 & 6 & 1,503 & 24,940 & 5,393 & 5,573 & 1,973 \\
\hline MCMASTER & 180 & 52 & 1,675 & 23,403 & 3,912 & 2,922 & 661 \\
\hline MANITOBA & 86 & 47 & 1,245 & 20,404 & 7,227 & 2,335 & 925 \\
\hline MARYLAND & 603 & 74 & 2,862 & 29,832 & 5,270 & 6,708 & 3,240 \\
\hline MASSACHUSETTS & 223 & 49 & 1,168 & 20,576 & 5,017 & 2,141 & 3,629 \\
\hline MIT & 602 & 35 & 1,122 & 9,970 & 236 & 5,955 & 185 \\
\hline MIAMI & 138 & 42 & 872 & 14,167 & 1,507 & 4,401 & 736 \\
\hline MICHIGAN & UA/NA & UA/NA & UA/NA & UA/NA & UA/NA & UA/NA & UA/NA \\
\hline MICHIGAN STATE & 463 & 111 & 1,893 & 39,881 & 6,237 & 7,681 & 2,759 \\
\hline MINNESOTA & 751 & 107 & 1,677 & 35,963 & 15,212 & 9,006 & 9,352 \\
\hline MISSOURI & 254 & 62 & 1,157 & 23,026 & 4,056 & 2,801 & 2,797 \\
\hline MONTREAL & 341 & 80 & 1,850 & 37,682 & 18,799 & 10,646 & 3,776 \\
\hline NEBRASKA & 245 & 41 & 1,041 & 18,433 & 3,242 & 2,665 & 1,973 \\
\hline NEW MEXICO & 181 & 37 & 1,742 & 18,659 & 8,274 & 2,642 & 2,449 \\
\hline NEW YORK & 415 & 72 & 3,202 & 30,157 & 9,847 & 11,176 & 8,262 \\
\hline NORTH CAROLINA & 490 & 69 & 1,382 & 22,441 & 4,835 & 6,530 & 3,982 \\
\hline NORTH CAROLINA STATE & 369 & 56 & 1,671 & 23,120 & 7,029 & 3,894 & 3,488 \\
\hline NORTHWESTERN & 423 & 58 & 2,563 & 15,013 & 3,073 & 6,902 & 1,946 \\
\hline NOTRE DAME & 162 & 24 & 877 & 11,239 & 178 & 2,979 & 163 \\
\hline $\mathrm{OHIO}$ & 124 & 56 & 1,092 & 23,922 & 4,829 & 2,706 & 828 \\
\hline OHIO STATE & 664 & 91 & 3,008 & 45,187 & 6,631 & 10,205 & 3,134 \\
\hline OKLAHOMA & 173 & 56 & 1,954 & 22,526 & 6,562 & 3,401 & 3,821 \\
\hline OKLAHOMA STATE & 150 & 39 & 1,154 & 23,780 & 11,895 & 2,007 & 3,622 \\
\hline OREGON & 150 & 42 & 802 & 17,145 & 3,249 & 2,903 & 1,013 \\
\hline PENNSYLVANIA & 435 & 70 & 1,388 & 19,771 & 3,933 & 6,978 & 2,263 \\
\hline PENNSYLVANIA STATE & 674 & 118 & 5,145 & 62,251 & 10,744 & 6,748 & 4,197 \\
\hline PITTSBURGH & 372 & 78 & 3,869 & 27,756 & 5,637 & 6,676 & 2,859 \\
\hline PRINCETON & 288 & 40 & 830 & 6,790 & 164 & 2,295 & 19 \\
\hline PURDUE & 566 & 59 & 1,960 & 34,968 & 3,744 & 5,772 & 2,065 \\
\hline QUEEN'S & 110 & 38 & 786 & 17,234 & 3,549 & 2,714 & 385 \\
\hline RICE & 137 & 31 & 597 & 4,973 & 122 & 1,916 & 67 \\
\hline ROCHESTER & 167 & 44 & 1,295 & 7,254 & 1,476 & 2,819 & 1,185 \\
\hline RUTGERS & 385 & 65 & 2,175 & 38,215 & 11,801 & 5,994 & 6,950 \\
\hline SASKATCHEWAN & 80 & 50 & 1,017 & 15,408 & 3,873 & 2,100 & 218 \\
\hline
\end{tabular}

* - Figures were reported on the ARL Questionnaire and have not been verified with the U.S. NCES IPEDS survey

** - Includes both undergraduate and graduate students

UA/NA - Unavailable or Not Applicable 


\section{Ph.D., Faculty, AND EnRollment Statistics *}

\begin{tabular}{|c|c|c|c|c|c|c|c|}
\hline \multirow{3}{*}{$\begin{array}{l}\text { (Survey Question \#) } \\
\text { INSTITUTION } \\
\end{array}$} & \multirow{3}{*}{$\begin{array}{r}\text { Ph.D.s } \\
\text { Awarded } \\
(36)\end{array}$} & \multirow{3}{*}{$\begin{array}{l}\text { Ph.D. } \\
\text { Fields } \\
\qquad(37)\end{array}$} & \multirow{3}{*}{$\begin{array}{r}\text { Faculty } \\
\text { (38) }\end{array}$} & \multicolumn{4}{|c|}{ ENROLLMENTS } \\
\hline & & & & \multirow{2}{*}{$\begin{array}{r}\text { Total FTE } \\
\text { Full-time }^{* *} \\
(39)\end{array}$} & \multirow{2}{*}{$\begin{array}{r}\text { Total } \\
\text { Part-time** }^{* *} \\
(40)\end{array}$} & \multirow{2}{*}{$\begin{array}{r}\text { Graduate FTE } \\
\text { Full-time } \\
(41)\end{array}$} & \multirow{2}{*}{$\begin{array}{r}\text { Graduate } \\
\text { Part-time } \\
\\
(42)\end{array}$} \\
\hline & & & & & & & \\
\hline SOUTH CAROLINA & 254 & 59 & 1,691 & 20,866 & 5,831 & 4,187 & 3,868 \\
\hline SOUTHERN CALIFORNIA & 411 & 80 & 1,876 & 28,823 & 4,013 & 12,751 & 3,188 \\
\hline SOUTHERN ILLINOIS & 139 & 35 & 901 & 17,297 & 4,144 & 2,335 & 2,409 \\
\hline SUNY-ALBANY & 132 & 39 & 608 & 14,014 & 3,420 & 2,394 & 2,583 \\
\hline SUNY-BUFFALO & 380 & 84 & 1,159 & 22,618 & 4,602 & 5,707 & 3,348 \\
\hline SUNY-STONY BROOK & 234 & 46 & 896 & 14,686 & 4,469 & 2,218 & 3,457 \\
\hline SYRACUSE & 164 & 45 & 927 & 16,793 & 2,690 & 4,665 & 1,923 \\
\hline TEMPLE & 387 & 52 & 1,682 & 28,835 & 7,893 & 4,698 & 4,803 \\
\hline TENNESSEE & 370 & 74 & 1,518 & 24,638 & 3,819 & 5,899 & 2,326 \\
\hline TEXAS & 701 & 91 & 2,467 & 45,614 & 4,763 & 11,726 & 1,274 \\
\hline TEXAS A\&M & 535 & 93 & 2,565 & 41,104 & 8,768 & 6,531 & 2,052 \\
\hline TEXAS TECH & 197 & 57 & 1,736 & 26,119 & 4,898 & 4,808 & 2,314 \\
\hline TORONTO & 626 & 98 & 2,637 & 59,901 & 8,046 & 9,921 & 2,040 \\
\hline TULANE & 105 & 43 & 1,395 & 10,080 & 1,827 & 4,724 & 491 \\
\hline UTAH & 276 & 55 & 1,214 & 20,335 & 8,677 & 3,820 & 1,555 \\
\hline VANDERBILT & 230 & 45 & 2,294 & 10,893 & 714 & 4,589 & 640 \\
\hline VIRGINIA & 301 & 46 & 1,121 & 19,383 & 4,382 & 5,988 & 3,564 \\
\hline VIRGINIA TECH & 366 & 48 & 1,304 & 25,178 & 2,801 & 4,091 & 2,261 \\
\hline WASHINGTON & 614 & 80 & 3,953 & 35,140 & 7,834 & 9,944 & 2,450 \\
\hline WASHINGTON STATE & 170 & 44 & 1,057 & 19,469 & 4,075 & 1,946 & 1,273 \\
\hline WASHINGTON U.-ST. LOUIS & 173 & 47 & 767 & 10,797 & 2,586 & 4,628 & 1,289 \\
\hline WATERLOO & 163 & 35 & 840 & 23,595 & 2,530 & 2,372 & 518 \\
\hline WAYNE STATE & 187 & 57 & 1,742 & 18,893 & 14,089 & 6,629 & 5,461 \\
\hline WESTERN ONTARIO & 192 & 49 & 1,279 & 24,625 & 3,477 & 3,540 & 420 \\
\hline WISCONSIN & 648 & 108 & 2,054 & 36,391 & 4,402 & 7,005 & 1,852 \\
\hline YALE & 329 & 61 & 1,756 & 11,276 & 207 & 5,926 & 148 \\
\hline YORK & 119 & 46 & 1,415 & 41,977 & 8,714 & 3,135 & 1,661 \\
\hline \multicolumn{8}{|c|}{ SUMMARY DATA: PH.D., FACULTY, AND ENROLlMENT STATISTICS } \\
\hline Median & 274 & 56 & 1,362 & 22,496 & 4,285 & 4,647 & 2,163 \\
\hline High & 799 & 118 & 5,145 & 62,251 & 18,799 & 14,026 & 9,352 \\
\hline Low & 70 & 6 & 597 & 4,973 & 43 & 1,466 & 15 \\
\hline Totals & 34,769 & 6,516 & 171,435 & $2,603,894$ & 555,813 & 568,968 & 258,267 \\
\hline \# of Libraries Responding & 112 & 111 & 112 & 112 & 112 & 112 & 112 \\
\hline
\end{tabular}

\footnotetext{
* - Figures were reported on the ARL Questionnaire and have not been verified with the U.S. NCES IPEDS survey

** - Includes both undergraduate and graduate students

UA/NA - Unavailable or Not Applicable
} 

RANK ORDER TABLES OF UNIVERSITY LIBRARIES, 2005-06 


\section{SUMMARY OF RANK ORDER TABLES FOR UNIVERSITY LIBRARIES, 2005-06}

The table below presents the rank for each university library in each of the eighteen categories for which rank order tables are prepared. The table numbers in the chart below refer to the data categories listed below. The number of libraries indicates the number of ARL university libraries supplying data in each category.

\section{TABLE DATA CATEGORY}

\section{VOLUMES IN LIBRARY}

VOLUMES ADDED (GROSS)

CURRENT SERIALS (TOTALS)

MICROFORM HOLDINGS

GOVERNMENT DOCUMENTS

MATERIALS EXPENDITURES

SALARIES \& WAGES EXPENDITURES

OTHER OPERATING EXPENDITURES

TOTAL LIBRARY EXPENDITURES

MONOGRAPHS PURCHASED (VOLS.)

EXPENDITURES FOR MONOGRAPHS
NO. OF LIBS.

TABLE DATA CATEGORY

SERIALS PURCHASED (SUBS.)

EXPENDITURES FOR SERIALS

TOTAL ITEMS LOANED (ILL)

TOTAL ITEMS BORROWED (ILL/DD)

PROFESSIONAL STAFF (FTE)

SUPPORT STAFF (FTE)

TOTAL STAFF (FTE)

E-MATERIALS AS \% OF TOTAL MATERIALS 113

EXPENDITURES-FOCUSED INDEX
EXPENDITURES FOR E-MATERIALS

NO. OF LIBS.

TABL:ES

\begin{tabular}{|c|c|c|c|c|c|c|c|c|c|c|c|c|c|c|c|c|c|c|c|c|c|}
\hline & 1 & 2 & 3 & 4 & 5 & 6 & 7 & 8 & 9 & 10 & 11 & 12 & 13 & 14 & 15 & 16 & 17 & 18 & 19 & 20 & 21 \\
\hline ALABAMA & 86 & 74 & 74 & 62 & 11 & 89 & 101 & 66 & 91 & 81 & 81 & 47 & 80 & 107 & 102 & 78 & 93 & 85 & 59 & 19 & 91 \\
\hline ALBERTA & 17 & 2 & 15 & 74 & 69 & 13 & 27 & 68 & 28 & UA & 9 & 15 & 19 & 8 & 25 & 55 & 14 & 36 & 5 & 34 & 27 \\
\hline ARIZONA & 23 & 35 & 75 & 25 & 69 & 33 & 35 & 20 & 32 & 28 & 38 & UA & 25 & 20 & 55 & 41 & 40 & 39 & 16 & 35 & 33 \\
\hline ARIZONA STATE & 33 & 33 & 77 & 7 & 16 & 37 & 38 & 56 & 40 & 13 & 27 & 67 & 34 & 28 & 47 & 52 & 23 & 37 & 30 & 50 & 40 \\
\hline AUBURN & 73 & 101 & 106 & 94 & 45 & 104 & 112 & 62 & 108 & 89 & 104 & 88 & 92 & 105 & 105 & 99 & 108 & 109 & 87 & 46 & 108 \\
\hline BOSTON & 95 & 59 & 55 & 47 & UA & 62 & 65 & 67 & 64 & 90 & 102 & UA & 74 & 97 & 96 & 41 & 75 & 43 & 103 & 104 & 65 \\
\hline BOSTON COLLEGE & 99 & 83 & 34 & 59 & 48 & 70 & 73 & 103 & 78 & UA & 72 & 29 & 58 & 93 & 92 & 62 & 96 & 90 & 105 & 100 & 74 \\
\hline BRIGHAM YOUNG & 39 & 23 & 85 & 70 & 33 & 55 & 42 & 39 & 45 & 5 & 52 & 66 & 42 & 53 & 22 & 23 & 106 & 25 & 100 & 99 & 43 \\
\hline BRITISH COLUMBIA & 20 & 14 & 30 & 42 & UA & 24 & 26 & 50 & 29 & UA & 50 & UA & 8 & 74 & 61 & 31 & 21 & 32 & 6 & 24 & 29 \\
\hline BROWN & 47 & 63 & 59 & 103 & 69 & 66 & 64 & 78 & 70 & 39 & 53 & 44 & 79 & 52 & 24 & 65 & 72 & 83 & 68 & 65 & 67 \\
\hline CALIFORNIA, BERKELEY & 5 & 7 & 3 & 14 & 26 & 7 & 2 & 12 & 5 & UA & 3 & 1 & 16 & 46 & 69 & 5 & 19 & 7 & UA & UA & 5 \\
\hline CALIFORNIA, DAVIS & 48 & 53 & 45 & 57 & 55 & 64 & 54 & 70 & 60 & UA & 32 & UA & 95 & 71 & 46 & 76 & 38 & 53 & 55 & 49 & 60 \\
\hline CALIFORNIA, IRVINE & 85 & 25 & 37 & 92 & 49 & 76 & 63 & 93 & 75 & 69 & 61 & 34 & 76 & 70 & 50 & 72 & 52 & 66 & 66 & 51 & 75 \\
\hline CALIFORNIA, LOS ANGELES & 9 & 29 & 8 & 28 & 56 & 12 & 6 & 3 & 6 & 17 & 5 & UA & 28 & 29 & 33 & 9 & 7 & 5 & 69 & 98 & 6 \\
\hline
\end{tabular}


INSTITUTION

TABL:ES

\begin{tabular}{|c|c|c|c|c|c|c|c|c|c|c|c|c|c|c|c|c|c|c|c|c|c|}
\hline & 1 & 2 & 3 & 4 & 5 & 6 & 7 & 8 & 9 & 10 & 11 & 12 & 13 & 14 & 15 & 16 & 17 & 18 & 19 & 20 & 21 \\
\hline CALIFORNIA, RIVERSIDE & 97 & 55 & 88 & 102 & 42 & 101 & 91 & 63 & 90 & 50 & 84 & 57 & 98 & 85 & 87 & 109 & 79 & 99 & 80 & 36 & 93 \\
\hline CALIFORNIA, SAN DIEGO & 61 & 27 & 80 & 83 & 69 & 73 & 29 & 23 & 38 & 8 & 40 & 64 & 78 & 64 & 51 & 54 & 28 & 35 & 65 & 52 & 41 \\
\hline CALIFORNIA, SANTA BARBARA & 75 & 91 & 68 & 73 & 32 & 108 & 68 & 18 & 71 & 71 & 77 & 63 & 106 & 82 & 37 & 105 & 44 & 67 & 94 & 56 & 78 \\
\hline CASE WESTERN RESERVE & 90 & 109 & 107 & 98 & 60 & 97 & 100 & 53 & 92 & 98 & 108 & 86 & 84 & 7 & 19 & 79 & 109 & 108 & 83 & 43 & 92 \\
\hline CHICAGO & 12 & 5 & 51 & 88 & UA & 9 & 36 & 41 & 24 & 4 & 12 & UA & 3 & 30 & 68 & 72 & 24 & 38 & 10 & 72 & 24 \\
\hline CINCINNATI & 63 & 34 & 52 & 81 & 59 & 46 & 49 & 55 & 52 & 40 & 30 & 27 & 50 & 5 & 14 & 35 & 102 & 69 & 3 & 2 & 49 \\
\hline COLORADO & 42 & 39 & 86 & 15 & 10 & 54 & 69 & 92 & 69 & 61 & 69 & 84 & 56 & 35 & 40 & 95 & 65 & 78 & 26 & 13 & 68 \\
\hline COLORADO STATE & 109 & 82 & 82 & 111 & 46 & 99 & 104 & 82 & 106 & 44 & 75 & 65 & 102 & 21 & 7 & 103 & 110 & 113 & 75 & 31 & 105 \\
\hline COLUMBIA & 6 & 6 & 2 & 26 & 50 & 4 & 5 & 11 & 4 & 3 & 7 & 2 & 1 & 31 & 15 & 2 & 10 & 3 & 2 & 71 & 3 \\
\hline CONNECTICUT & 44 & 97 & 29 & 52 & UA & 75 & 28 & 65 & 44 & 62 & 97 & 26 & 53 & 39 & 49 & 52 & 98 & 75 & 62 & 47 & 44 \\
\hline CORNELL & 11 & 18 & 9 & 5 & UA & 14 & 10 & 9 & 9 & UA & 8 & 9 & 36 & 19 & 32 & 26 & 5 & 10 & 22 & 69 & 10 \\
\hline DARTMOUTH & 83 & 80 & 31 & 96 & 69 & 80 & 76 & 81 & 77 & 65 & 56 & 21 & 68 & 60 & 45 & 83 & 60 & 88 & 44 & 16 & 79 \\
\hline DELAWARE & 82 & 95 & 110 & 79 & 30 & 71 & 79 & 84 & 84 & 63 & 49 & 90 & 82 & 96 & 62 & 85 & 66 & 85 & 32 & 5 & 83 \\
\hline DUKE & 22 & 31 & 28 & 58 & 6 & 19 & 23 & 19 & 20 & 16 & 18 & 11 & 14 & 22 & 43 & 10 & 37 & 30 & 39 & 78 & 18 \\
\hline EMORY & 64 & 36 & 63 & 29 & 41 & 17 & 31 & 33 & 27 & 36 & 13 & 51 & 63 & 24 & 84 & 36 & 41 & 45 & 77 & 102 & 25 \\
\hline FLORIDA & 36 & 76 & 5 & 6 & 5 & 40 & 32 & 31 & 35 & 52 & 64 & 6 & 20 & 68 & 77 & 32 & 19 & 17 & 23 & 26 & 35 \\
\hline FLORIDA STATE & 74 & 58 & 38 & 3 & 14 & 82 & 85 & 111 & 89 & 75 & 82 & 19 & 91 & 87 & 89 & 68 & 55 & 64 & 52 & 23 & 89 \\
\hline GEORGE WASHINGTON & 105 & 86 & 94 & 91 & 69 & 53 & 60 & 46 & 54 & 70 & 31 & 62 & 70 & 58 & 9 & 60 & 50 & 56 & 82 & 83 & 54 \\
\hline GEORGETOWN & 91 & 50 & 84 & 65 & UA & 41 & 47 & 58 & 46 & 26 & 33 & 46 & 44 & 51 & 63 & 51 & 51 & 54 & 74 & 90 & 45 \\
\hline GEORGIA & 32 & 48 & 61 & 18 & 9 & 38 & 56 & 69 & 50 & 31 & 35 & 43 & 30 & 33 & 106 & 65 & 26 & 46 & 18 & 29 & 52 \\
\hline GEORGIA TECH & 96 & 96 & 73 & 49 & 69 & 106 & 111 & 97 & 111 & 101 & 110 & 80 & 103 & 108 & 107 & 105 & 100 & 112 & UA & UA & 110 \\
\hline GUELPH & 113 & 113 & 111 & 100 & 69 & 111 & 107 & 99 & 110 & 94 & 96 & 85 & 108 & 38 & 6 & 110 & 99 & 111 & 72 & 4 & 111 \\
\hline HARVARD & 1 & 1 & 4 & 2 & UA & 2 & 1 & 1 & 1 & UA & 2 & UA & 7 & 41 & 98 & 1 & 1 & 1 & 20 & 105 & 1 \\
\hline HAWAII & 54 & 65 & 76 & 77 & 69 & 83 & 74 & 91 & 80 & 58 & 58 & 68 & 69 & 112 & 85 & 60 & 85 & 74 & 88 & 75 & 81 \\
\hline HOUSTON & 104 & 40 & 101 & 31 & 19 & 68 & 97 & 49 & 82 & 34 & 71 & 70 & 67 & 67 & 79 & 79 & 85 & 91 & 92 & 89 & 84 \\
\hline HOWARD & 98 & 105 & 112 & 72 & 65 & 113 & 108 & 95 & 113 & 102 & 111 & 89 & 111 & 110 & 109 & 95 & 100 & 100 & 109 & 81 & 113 \\
\hline ILLINOIS, CHICAGO & 100 & 87 & 41 & 64 & 17 & 67 & 78 & 75 & 73 & 66 & 73 & UA & 54 & 9 & 26 & 57 & 63 & 72 & 64 & 58 & 73 \\
\hline ILLINOIS, URBANA & 4 & 10 & 19 & 4 & 69 & 31 & 14 & 22 & 16 & 18 & 37 & 8 & 17 & 6 & 2 & 8 & 22 & 13 & 70 & 92 & 16 \\
\hline INDIANA & 13 & 11 & 7 & 40 & 38 & 20 & 25 & 35 & 22 & 19 & 10 & 5 & 37 & 4 & 12 & 12 & 26 & 14 & 46 & 86 & 21 \\
\hline
\end{tabular}


TABL:ES

\begin{tabular}{|c|c|c|c|c|c|c|c|c|c|c|c|c|c|c|c|c|c|c|c|c|c|}
\hline & 1 & 2 & 3 & 4 & 5 & 6 & 7 & 8 & 9 & 10 & 11 & 12 & 13 & 14 & 15 & 16 & 17 & 18 & 19 & 20 & 21 \\
\hline IOWA & 31 & 28 & 39 & 12 & 22 & 26 & 39 & 87 & 41 & 20 & 41 & 38 & 35 & 16 & 53 & 50 & 45 & 43 & 19 & 53 & 38 \\
\hline IOWA STATE & 94 & 79 & 83 & 78 & 69 & 59 & 83 & 106 & 83 & 67 & 67 & 72 & 51 & 62 & 97 & 99 & 85 & 94 & 12 & 3 & 82 \\
\hline JOHNS HOPKINS & 41 & 71 & 10 & 53 & 68 & 22 & 48 & 28 & 34 & 11 & 34 & 22 & 11 & 54 & 29 & 18 & 36 & 33 & 8 & 37 & 32 \\
\hline KANSAS & 35 & 49 & 43 & 75 & 18 & 69 & 53 & 48 & 59 & 45 & 62 & 48 & 73 & 25 & 18 & 36 & 66 & 48 & 89 & 84 & 58 \\
\hline KENT STATE & 79 & 85 & 109 & UA & 69 & 112 & 113 & 52 & 112 & 100 & 107 & 82 & 110 & 103 & 108 & 93 & 112 & 49 & 102 & 32 & 112 \\
\hline KENTUCKY & 55 & 38 & 81 & 21 & 7 & 52 & 75 & 71 & 63 & 51 & 76 & 60 & 40 & 66 & 60 & 48 & 59 & 58 & 63 & 70 & 63 \\
\hline LAVAL & 78 & 61 & 91 & 109 & 69 & 60 & 67 & 112 & 76 & 57 & 48 & 52 & 52 & 91 & 100 & 85 & 38 & 72 & 40 & 28 & 76 \\
\hline LOUISIANA STATE & 51 & 67 & 24 & 16 & 24 & 94 & 109 & 104 & 107 & 84 & 68 & UA & 93 & 109 & 65 & 93 & 79 & 89 & 107 & 97 & 107 \\
\hline LOUISVILLE & 108 & 54 & 62 & 101 & 69 & 57 & 95 & 40 & 72 & 22 & 103 & 31 & 24 & 86 & 39 & 99 & 89 & 93 & 85 & 87 & 71 \\
\hline MCGILL & 43 & 47 & 6 & 104 & 21 & 35 & 46 & 34 & 39 & 33 & 20 & 3 & 47 & 94 & 74 & 79 & 34 & 60 & 11 & 18 & 39 \\
\hline MCMASTER & 111 & 110 & 93 & 106 & 69 & 90 & 102 & 105 & 104 & 54 & 83 & 73 & 90 & 98 & 95 & 113 & 69 & 103 & 43 & 6 & 102 \\
\hline MANITOBA & 106 & 94 & 90 & 108 & 47 & 92 & 59 & 47 & 67 & 32 & 63 & 61 & 97 & 88 & 41 & 83 & 53 & 75 & 73 & 39 & 69 \\
\hline MARYLAND & 50 & 56 & 79 & 33 & 51 & 63 & 40 & 36 & 47 & 25 & 57 & 81 & 94 & 95 & 20 & 15 & 78 & 15 & 50 & 44 & 48 \\
\hline MASSACHUSETTS & 62 & 106 & 56 & 97 & UA & 103 & 81 & 76 & 95 & 88 & 100 & 36 & 101 & 23 & 30 & 92 & 97 & 96 & 86 & 38 & 97 \\
\hline MIT & 76 & 90 & 100 & 99 & UA & 72 & 37 & 85 & 56 & 82 & 87 & 83 & 55 & 106 & 101 & 43 & 82 & 81 & 71 & 64 & 55 \\
\hline MIAMI & 72 & 45 & 40 & 66 & 69 & 32 & 66 & 51 & 48 & 53 & 43 & 18 & 15 & 47 & 90 & 55 & 57 & 58 & 53 & 79 & 47 \\
\hline MICHIGAN & 8 & 8 & 1 & UA & UA & 5 & 8 & UA & 7 & UA & UA & 4 & UA & UA & UA & 11 & 6 & 9 & UA & UA & 7 \\
\hline MICHIGAN STATE & 29 & 42 & 59 & 17 & 69 & 48 & 44 & 44 & 42 & 49 & 47 & 55 & 38 & 37 & 31 & 58 & 58 & 47 & 33 & 27 & 46 \\
\hline MINNESOTA & 15 & 15 & 12 & 13 & 2 & 15 & 18 & 10 & 15 & 43 & 28 & 16 & 9 & 1 & 28 & 34 & 15 & 21 & 21 & 66 & 15 \\
\hline MISSOURI & 57 & 77 & 71 & 10 & 3 & 96 & 93 & 74 & 97 & 47 & 85 & 69 & 89 & 17 & 21 & 85 & 60 & 80 & 84 & 55 & 98 \\
\hline MONTREAL & 68 & 57 & 23 & 107 & 69 & 45 & 19 & 94 & 33 & 24 & 59 & 13 & 23 & 73 & 76 & 33 & 9 & 24 & 29 & 30 & 34 \\
\hline NEBRASKA & 67 & 73 & 35 & 46 & 25 & 88 & 86 & 61 & 86 & 92 & 94 & 23 & 77 & 72 & 75 & 95 & 64 & 81 & 97 & 85 & 87 \\
\hline NEW MEXICO & 84 & 81 & 95 & 45 & 23 & 107 & 45 & 43 & 66 & 80 & 106 & 74 & 104 & 81 & 67 & 64 & 33 & 42 & 95 & 59 & 70 \\
\hline NEW YORK & 24 & 13 & 21 & 22 & 28 & 10 & 16 & 8 & 11 & 10 & 15 & 25 & 4 & 76 & 52 & 21 & 12 & 16 & 7 & 61 & 11 \\
\hline NORTH CAROLINA & 21 & 24 & 33 & 44 & 4 & 18 & 20 & 32 & 18 & 14 & 24 & 39 & 22 & 27 & 94 & 14 & 18 & 19 & 54 & 91 & 17 \\
\hline NORTH CAROLINA STATE & 40 & 9 & 32 & 36 & 69 & 58 & 50 & 5 & 36 & 56 & 66 & 54 & 64 & 104 & 59 & 27 & 70 & 52 & 91 & 93 & 37 \\
\hline NORTHWESTERN & 30 & 30 & 50 & 51 & 62 & 29 & 33 & 73 & 37 & 35 & 23 & UA & 27 & 45 & 44 & 28 & 42 & 34 & 14 & 33 & 36 \\
\hline NOTRE DAME & 60 & 43 & 103 & 68 & UA & 50 & 55 & 57 & 55 & 23 & 22 & UA & 85 & 79 & 86 & 70 & 35 & 57 & 41 & 48 & 56 \\
\hline $\mathrm{OHIO}$ & 80 & 52 & 102 & 82 & UA & 105 & 98 & 59 & 103 & 48 & 90 & 77 & 107 & 10 & 11 & 77 & 103 & 95 & 90 & 42 & 104 \\
\hline
\end{tabular}


TABL:ES

\begin{tabular}{|c|c|c|c|c|c|c|c|c|c|c|c|c|c|c|c|c|c|c|c|c|c|}
\hline & 1 & 2 & 3 & 4 & 5 & 6 & 7 & 8 & 9 & 10 & 11 & 12 & 13 & 14 & 15 & 16 & 17 & 18 & 19 & 20 & 21 \\
\hline OHIO STATE & 18 & 20 & 69 & 32 & 66 & 28 & 22 & 17 & 21 & 6 & 21 & 58 & 43 & 3 & 1 & 21 & 29 & 20 & 27 & 57 & 22 \\
\hline OKLAHOMA & 27 & 37 & 18 & 54 & 61 & 30 & 96 & 83 & 62 & 64 & 29 & 32 & 59 & 56 & 35 & 85 & 76 & 69 & 79 & 96 & 61 \\
\hline OKLAHOMA STATE & 81 & 32 & 20 & 50 & 52 & 91 & 105 & 79 & 101 & 68 & UA & UA & UA & 49 & 66 & 79 & 95 & 79 & UA & UA & 101 \\
\hline OREGON & 77 & 93 & 98 & 90 & 29 & 110 & 84 & 88 & 102 & 37 & 86 & 75 & 109 & 12 & 5 & 91 & 83 & 83 & 101 & 74 & 103 \\
\hline PENNSYLVANIA & 19 & 26 & 44 & 60 & UA & 21 & 21 & 21 & 19 & UA & 19 & UA & 6 & 15 & 3 & 23 & 32 & 23 & 25 & 67 & 20 \\
\hline PENNSYLVANIA STATE & 26 & 41 & 14 & 39 & 69 & 6 & 7 & 7 & 8 & 12 & 17 & 14 & 2 & 18 & 13 & 13 & 2 & 6 & 1 & 14 & 8 \\
\hline PITTSBURGH & 28 & 17 & 42 & 9 & UA & 23 & 34 & 42 & 31 & UA & 25 & UA & 26 & 14 & 64 & 23 & 30 & 29 & 13 & 54 & 31 \\
\hline PRINCETON & 16 & 16 & 53 & 20 & 54 & 8 & 17 & 29 & 13 & 9 & 6 & 28 & 33 & 59 & 36 & 16 & 17 & 22 & 31 & 88 & 13 \\
\hline PURDUE & 89 & 64 & 70 & 86 & 27 & 39 & 52 & 45 & 43 & 78 & 42 & 79 & 49 & 65 & 54 & 72 & 55 & 62 & 98 & 106 & 42 \\
\hline QUEEN'S & 92 & 89 & 49 & 67 & 8 & 74 & 88 & 72 & 85 & 74 & 89 & 30 & 60 & 83 & 99 & 108 & 68 & 101 & 38 & 12 & 86 \\
\hline RICE & 93 & 84 & 108 & 85 & 58 & 49 & 110 & 98 & 87 & 46 & 16 & 87 & 81 & 102 & 103 & 90 & 106 & 110 & 47 & 60 & 85 \\
\hline ROCHESTER & 45 & 78 & 92 & 43 & 31 & 93 & 72 & 60 & 79 & 87 & 95 & UA & 83 & 75 & 71 & 48 & 105 & 77 & 104 & 95 & 77 \\
\hline RUTGERS & 34 & 51 & 36 & 30 & 1 & 43 & 15 & 64 & 26 & 77 & 74 & 20 & 31 & 61 & 56 & 36 & 11 & 18 & 56 & 73 & 28 \\
\hline SASKATCHEWAN & 112 & 104 & 66 & 87 & 34 & 79 & 99 & 102 & 94 & 85 & 44 & 45 & 75 & 80 & 73 & 107 & 83 & 107 & 49 & 22 & 90 \\
\hline SOUTH CAROLINA & 49 & 68 & 27 & 41 & 12 & 87 & 77 & 16 & 61 & 30 & 91 & 78 & 65 & 90 & 81 & 69 & 74 & 51 & 57 & 20 & 64 \\
\hline SOUTHERN CALIFORNIA & 37 & 72 & 25 & 27 & 43 & 25 & 24 & 27 & 23 & 59 & 26 & 12 & 41 & 77 & 91 & 29 & 49 & 26 & 67 & 94 & 23 \\
\hline SOUTHERN ILLINOIS & 71 & 98 & 58 & 48 & 39 & 86 & 87 & 108 & 96 & 38 & 105 & 56 & 66 & 26 & 27 & 95 & 85 & 85 & 78 & 63 & 94 \\
\hline SUNY-ALBANY & 107 & 103 & 96 & 93 & 69 & 109 & 92 & 110 & 109 & 95 & 101 & 76 & 105 & 101 & 83 & 85 & 104 & 105 & 106 & 80 & 109 \\
\hline SUNY-BUFFALO & 53 & 92 & 65 & 37 & UA & 78 & 51 & 86 & 65 & 96 & 98 & 53 & 57 & 43 & 88 & 19 & 111 & 63 & 36 & 7 & 62 \\
\hline SUNY-STONY BROOK & 103 & 111 & 64 & 71 & 37 & 95 & 89 & 100 & 99 & 99 & 109 & 41 & 86 & 78 & 93 & 46 & 113 & 105 & 96 & 76 & 95 \\
\hline SYRACUSE & 66 & 107 & 99 & 11 & 40 & 98 & 70 & 96 & 88 & 86 & 79 & 71 & 100 & 100 & 72 & 70 & 48 & 69 & 81 & 41 & 88 \\
\hline TEMPLE & 70 & 69 & 89 & 84 & 67 & 61 & 80 & 77 & 74 & 27 & 51 & 59 & 88 & 84 & 80 & 62 & 79 & 68 & 28 & 9 & 72 \\
\hline TENNESSEE & 65 & 66 & 78 & 56 & 63 & 36 & 58 & 80 & 53 & 97 & 80 & 49 & 13 & 55 & 48 & 46 & 42 & 49 & 93 & 103 & 50 \\
\hline TEXAS & 7 & 19 & 46 & 19 & 69 & 11 & 13 & 14 & 10 & 15 & 11 & UA & 18 & 36 & 34 & 16 & 8 & 11 & 9 & 68 & 9 \\
\hline TEXAS A\&M & 46 & 12 & 48 & 34 & 57 & 16 & 43 & 30 & 30 & 7 & 14 & 35 & 12 & 42 & 8 & 20 & 45 & 28 & 4 & 21 & 30 \\
\hline TEXAS TECH & 88 & 60 & 26 & 95 & 64 & 51 & 71 & 37 & 57 & 41 & 60 & 33 & 45 & 63 & 57 & 39 & 60 & 41 & 99 & 101 & 57 \\
\hline TORONTO & 3 & 4 & 16 & 38 & UA & 3 & 4 & 13 & 3 & 2 & 4 & 10 & 5 & 57 & 104 & 7 & 4 & 4 & 51 & 107 & 4 \\
\hline TULANE & 69 & 100 & 105 & 110 & 53 & 81 & 103 & 107 & 98 & 76 & 92 & UA & 87 & 111 & 110 & 99 & 93 & 98 & 60 & 40 & 96 \\
\hline UTAH & 59 & 46 & 57 & 76 & 13 & 84 & 41 & 24 & 49 & UA & 65 & UA & 72 & 48 & 58 & 59 & 25 & 31 & 108 & 108 & 53 \\
\hline
\end{tabular}


INSTITUTION

TABL:ES

\begin{tabular}{lccccccccccccccccccccccccc}
\hline & 1 & 2 & 3 & 4 & 5 & 6 & 7 & 8 & 9 & 10 & 11 & 12 & 13 & 14 & 15 & 16 & 17 & 18 & 19 & 20 & 21 \\
\hline VANDERBILT & 58 & 70 & 72 & 89 & 44 & 44 & 61 & 38 & 51 & 60 & 55 & 42 & 21 & 69 & 78 & 39 & 70 & 65 & 34 & 45 & 51 \\
VIRGINIA & 25 & 44 & 13 & 35 & 20 & 47 & 12 & 25 & 17 & 42 & 46 & 50 & 46 & 44 & 38 & 43 & 13 & 27 & 45 & 62 & 19 \\
VIRGINIA TECH & 102 & 102 & 67 & 23 & 35 & 85 & 106 & 90 & 100 & 73 & 78 & 37 & 71 & 89 & 70 & 111 & 89 & 104 & 42 & 11 & 100 \\
WASHINGTON & 14 & 22 & 22 & 8 & 69 & 27 & 11 & 15 & 14 & 21 & 39 & UA & 10 & 11 & 10 & 6 & 16 & 12 & 48 & 77 & 14 \\
WASHINGTON STATE & 101 & 108 & 87 & 55 & 69 & 102 & 90 & 89 & 105 & 93 & 99 & UA & 99 & 40 & 82 & 103 & 92 & 101 & 76 & 17 & 106 \\
WASHINGTON U.-ST. LOUIS & 38 & 62 & 54 & 80 & 69 & 42 & 30 & 4 & 25 & 79 & 54 & 40 & 39 & 34 & 16 & 29 & 47 & 40 & 24 & 25 & 26 \\
WATERLOO & 110 & 112 & 104 & 105 & 69 & 100 & 94 & 54 & 93 & 83 & 93 & UA & 96 & 13 & 17 & 112 & 76 & 96 & 61 & 10 & 99 \\
WAYNE STATE & 52 & 99 & 97 & 69 & 36 & 77 & 62 & 26 & 58 & 91 & 88 & UA & 61 & 50 & 42 & 45 & 89 & 55 & 15 & 1 & 59 \\
WESTERN ONTARIO & 56 & 75 & 47 & 63 & 69 & 56 & 82 & 109 & 81 & 72 & 70 & 24 & 32 & 92 & 111 & 65 & 73 & 92 & 17 & 8 & 80 \\
WISCONSIN & 10 & 21 & 17 & 24 & 15 & 34 & 9 & 6 & 12 & 29 & 36 & 17 & 48 & 2 & 4 & 4 & 30 & 8 & 58 & 82 & 12 \\
YALE & 2 & 3 & 11 & 1 & 69 & 1 & 3 & 2 & 2 & 1 & 1 & 7 & 29 & 32 & 23 & 3 & 3 & 2 & 35 & 109 & 2 \\
YORK & 87 & 88 & 113 & 61 & UA & 65 & 57 & 101 & 68 & 55 & 45 & UA & 62 & 99 & 112 & 72 & 53 & 61 & 37 & 15 & 66
\end{tabular}




\section{RANK ORDER TABLE 1 VOLUMES IN LIBRARY}

\begin{tabular}{|c|c|c|c|c|c|}
\hline RANK & INSTITUTION & VALUE & RANK & INSTITUTION & VALUE \\
\hline 1 & HARVARD & $15,826,570$ & 58 & VANDERBILT & $3,264,231$ \\
\hline 2 & YALE & $12,368,757$ & 59 & UTAH & $3,254,709$ \\
\hline 3 & TORONTO & $10,536,868$ & 60 & NOTRE DAME & $3,246,968$ \\
\hline 4 & ILLINOIS, URBANA & $10,524,935$ & 61 & CALIFORNIA, SAN DIEGO & $3,236,219$ \\
\hline 5 & CALIFORNIA, BERKELEY & $10,094,417$ & 62 & MASSACHUSETTS & $3,230,697$ \\
\hline 6 & COLUMBIA & $9,455,312$ & 63 & CINCINNATI & $3,209,337$ \\
\hline 7 & TEXAS & $9,022,363$ & 64 & EMORY & $3,184,754$ \\
\hline 8 & MICHIGAN & $8,273,050$ & 65 & TENNESSEE & $3,183,529$ \\
\hline 9 & CALIFORNIA, LOS ANGELES & $8,157,182$ & 66 & SYRACUSE & $3,180,427$ \\
\hline 10 & WISCONSIN & $8,015,081$ & 67 & NEBRASKA & $3,113,473$ \\
\hline 11 & CORNELL & $7,785,263$ & 68 & MONTREAL & $3,090,289$ \\
\hline 12 & CHICAGO & $7,765,583$ & 69 & TULANE & $3,076,954$ \\
\hline 13 & INDIANA & $7,374,784$ & 70 & TEMPLE & $3,071,624$ \\
\hline 14 & WASHINGTON & $7,111,065$ & 71 & SOUTHERN ILLINOIS & $3,026,125$ \\
\hline 15 & MINNESOTA & $6,713,629$ & 72 & MIAMI & $3,013,867$ \\
\hline 16 & PRINCETON & $6,618,464$ & 73 & AUBURN & $2,984,279$ \\
\hline 17 & ALBERTA & $6,416,254$ & 74 & FLORIDA STATE & $2,947,702$ \\
\hline 18 & OHIO STATE & $6,180,744$ & 75 & CALIFORNIA, SANTA BARBARA & $2,880,294$ \\
\hline 19 & PENNSYLVANIA & $5,880,460$ & 76 & MIT & $2,807,568$ \\
\hline 20 & BRITISH COLUMBIA & $5,820,527$ & 77 & OREGON & $2,787,476$ \\
\hline 21 & NORTH CAROLINA & $5,816,677$ & 78 & LAVAL & $2,785,210$ \\
\hline 22 & DUKE & $5,665,241$ & 79 & KENT STATE & $2,758,324$ \\
\hline 23 & ARIZONA & $5,533,482$ & 80 & $\mathrm{OHIO}$ & $2,722,197$ \\
\hline 24 & NEW YORK & $5,144,879$ & 81 & OKLAHOMA STATE & $2,711,255$ \\
\hline 25 & VIRGINIA & $5,102,954$ & 82 & DELAWARE & $2,704,986$ \\
\hline 26 & PENNSYLVANIA STATE & $5,069,854$ & 83 & DARTMOUTH & $2,690,395$ \\
\hline 27 & OKLAHOMA & $4,918,351$ & 84 & NEW MEXICO & $2,675,082$ \\
\hline 28 & PITTSBURGH & $4,909,264$ & 85 & CALIFORNIA, IRVINE & $2,605,003$ \\
\hline 29 & MICHIGAN STATE & $4,864,603$ & 86 & ALABAMA & $2,566,978$ \\
\hline 30 & NORTHWESTERN & $4,687,828$ & 87 & YORK & $2,526,324$ \\
\hline 31 & IOWA & $4,592,560$ & 88 & TEXAS TECH & $2,525,957$ \\
\hline 32 & GEORGIA & $4,345,939$ & 89 & PURDUE & $2,511,097$ \\
\hline 33 & ARIZONA STATE & $4,254,719$ & 90 & CASE WESTERN RESERVE & $2,495,769$ \\
\hline 34 & RUTGERS & $4,203,237$ & 91 & GEORGETOWN & $2,489,528$ \\
\hline 35 & KANSAS & $4,194,283$ & 92 & QUEEN'S & $2,477,137$ \\
\hline 36 & FLORIDA & $4,178,355$ & 93 & RICE & $2,474,352$ \\
\hline 37 & SOUTHERN CALIFORNIA & $3,968,814$ & 94 & IOWA STATE & $2,473,075$ \\
\hline 38 & WASHINGTON U.-ST. LOUIS & $3,947,725$ & 95 & BOSTON & $2,449,521$ \\
\hline 39 & BRIGHAM YOUNG & $3,738,847$ & 96 & GEORGIA TECH & $2,449,323$ \\
\hline 40 & NORTH CAROLINA STATE & $3,687,733$ & 97 & CALIFORNIA, RIVERSIDE & $2,435,296$ \\
\hline 41 & JOHNS HOPKINS & $3,686,575$ & 98 & HOWARD & $2,435,163$ \\
\hline 42 & COLORADO & $3,641,096$ & 99 & BOSTON COLLEGE & $2,407,253$ \\
\hline 43 & MCGILL & $3,631,326$ & 100 & ILLINOIS, CHICAGO & $2,324,857$ \\
\hline 44 & CONNECTICUT & $3,629,755$ & 101 & WASHINGTON STATE & $2,277,967$ \\
\hline 45 & ROCHESTER & $3,607,310$ & 102 & VIRGINIA TECH & $2,268,619$ \\
\hline 46 & TEXAS A\&M & $3,581,226$ & 103 & SUNY-STONY BROOK & $2,237,823$ \\
\hline 47 & BROWN & $3,568,944$ & 104 & HOUSTON & $2,231,199$ \\
\hline 48 & CALIFORNIA, DAVIS & $3,549,004$ & 105 & GEORGE WASHINGTON & $2,197,950$ \\
\hline 49 & SOUTH CAROLINA & $3,532,538$ & 106 & MANITOBA & $2,190,304$ \\
\hline 50 & MARYLAND & $3,501,054$ & 107 & SUNY-ALBANY & $2,129,103$ \\
\hline 51 & LOUISIANA STATE & $3,499,137$ & 108 & LOUISVILLE & $2,081,825$ \\
\hline 52 & WAYNE STATE & $3,443,247$ & 109 & COLORADO STATE & $2,056,928$ \\
\hline 53 & SUNY-BUFFALO & $3,423,148$ & 110 & WATERLOO & $2,008,232$ \\
\hline 54 & HAWAII & $3,407,167$ & 111 & MCMASTER & $2,005,280$ \\
\hline 55 & KENTUCKY & $3,406,377$ & 112 & SASKATCHEWAN & $1,996,901$ \\
\hline 56 & WESTERN ONTARIO & $3,388,376$ & 113 & GUELPH & $1,557,742$ \\
\hline 57 & MISSOURI & $3,295,378$ & & & \\
\hline
\end{tabular}




\section{RANK ORDER TABLE 2 VOLUMES ADDED (GROSS)}

\begin{tabular}{|c|c|c|c|c|c|}
\hline RANK & INSTITUTION & VALUE & RANK & INSTITUTION & VALUE \\
\hline 1 & HARVARD & 292,658 & 58 & FLORIDA STATE & 64,265 \\
\hline 2 & ALBERTA & 255,975 & 59 & BOSTON & 63,186 \\
\hline 3 & YALE & 249,613 & 60 & TEXAS TECH & 63,179 \\
\hline 4 & TORONTO & 216,531 & 61 & LAVAL & 60,462 \\
\hline 5 & CHICAGO & 208,122 & 62 & WASHINGTON U.-ST. LOUIS & 60,029 \\
\hline 6 & COLUMBIA & 189,507 & 63 & BROWN & 59,889 \\
\hline 7 & CALIFORNIA, BERKELEY & 186,987 & 64 & PURDUE & 58,618 \\
\hline 8 & MICHIGAN & 176,998 & 65 & HAWAII & 58,083 \\
\hline 9 & NORTH CAROLINA STATE & 159,765 & 66 & TENNESSEE & 57,953 \\
\hline 10 & ILLINOIS, URBANA & 158,914 & 67 & LOUISIANA STATE & 57,772 \\
\hline 11 & INDIANA & 155,895 & 68 & SOUTH CAROLINA & 56,298 \\
\hline 12 & TEXAS A\&M & 149,398 & 69 & TEMPLE & 55,830 \\
\hline 13 & NEW YORK & 137,629 & 70 & VANDERBILT & 55,283 \\
\hline 14 & BRITISH COLUMBIA & 133,885 & 71 & JOHNS HOPKINS & 53,306 \\
\hline 15 & MINNESOTA & 132,628 & 72 & SOUTHERN CALIFORNIA & 53,232 \\
\hline 16 & PRINCETON & 130,801 & 73 & NEBRASKA & 52,880 \\
\hline 17 & PITTSBURGH & 129,443 & 74 & ALABAMA & 52,804 \\
\hline 18 & CORNELL & 127,998 & 75 & WESTERN ONTARIO & 52,337 \\
\hline 19 & TEXAS & 127,845 & 76 & FLORIDA & 51,388 \\
\hline 20 & OHIO STATE & 123,424 & 77 & MISSOURI & 51,329 \\
\hline 21 & WISCONSIN & 121,148 & 78 & ROCHESTER & 49,221 \\
\hline 22 & WASHINGTON & 120,950 & 79 & IOWA STATE & 48,314 \\
\hline 23 & BRIGHAM YOUNG & 120,649 & 80 & DARTMOUTH & 48,139 \\
\hline 24 & NORTH CAROLINA & 118,374 & 81 & NEW MEXICO & 47,699 \\
\hline 25 & CALIFORNIA, IRVINE & 118,034 & 82 & COLORADO STATE & 46,823 \\
\hline 26 & PENNSYLVANIA & 108,678 & 83 & BOSTON COLLEGE & 46,782 \\
\hline 27 & CALIFORNIA, SAN DIEGO & 108,376 & 84 & RICE & 46,741 \\
\hline 28 & IOWA & 107,046 & 85 & KENT STATE & 46,660 \\
\hline 29 & CALIFORNIA, LOS ANGELES & 106,767 & 86 & GEORGE WASHINGTON & 46,613 \\
\hline 30 & NORTHWESTERN & 102,557 & 87 & ILLINOIS, CHICAGO & 46,373 \\
\hline 31 & DUKE & 101,563 & 88 & YORK & 46,039 \\
\hline 32 & OKLAHOMA STATE & 92,050 & 89 & QUEEN'S & 45,541 \\
\hline 33 & ARIZONA STATE & 89,216 & 90 & MIT & 44,170 \\
\hline 34 & CINCINNATI & 87,840 & 91 & CALIFORNIA, SANTA BARBARA & 43,820 \\
\hline 35 & ARIZONA & 87,095 & 92 & SUNY-BUFFALO & 41,442 \\
\hline 36 & EMORY & 86,754 & 93 & OREGON & 40,872 \\
\hline 37 & OKLAHOMA & 84,137 & 94 & MANITOBA & 40,744 \\
\hline 38 & KENTUCKY & 83,729 & 95 & DELAWARE & 40,076 \\
\hline 39 & COLORADO & 82,741 & 96 & GEORGIA TECH & 39,428 \\
\hline 40 & HOUSTON & 80,780 & 97 & CONNECTICUT & 38,920 \\
\hline 41 & PENNSYLVANIA STATE & 80,745 & 98 & SOUTHERN ILLINOIS & 36,947 \\
\hline 42 & MICHIGAN STATE & 80,572 & 99 & WAYNE STATE & 36,636 \\
\hline 43 & NOTRE DAME & 76,470 & 100 & TULANE & 35,843 \\
\hline 44 & VIRGINIA & 76,325 & 101 & AUBURN & 35,713 \\
\hline 45 & MIAMI & 76,138 & 102 & VIRGINIA TECH & 35,702 \\
\hline 46 & UTAH & 76,048 & 103 & SUNY-ALBANY & 35,590 \\
\hline 47 & MCGILL & 75,737 & 104 & SASKATCHEWAN & 35,466 \\
\hline 48 & GEORGIA & 75,195 & 105 & HOWARD & 34,150 \\
\hline 49 & KANSAS & 74,974 & 106 & MASSACHUSETTS & 33,462 \\
\hline 50 & GEORGETOWN & 74,321 & 107 & SYRACUSE & 32,087 \\
\hline 51 & RUTGERS & 72,957 & 108 & WASHINGTON STATE & 29,752 \\
\hline 52 & OHIO & 71,231 & 109 & CASE WESTERN RESERVE & 28,365 \\
\hline 53 & CALIFORNIA, DAVIS & 68,375 & 110 & MCMASTER & 27,495 \\
\hline 54 & LOUISVILLE & 67,393 & 111 & SUNY-STONY BROOK & 26,287 \\
\hline 55 & CALIFORNIA, RIVERSIDE & 66,733 & 112 & WATERLOO & 23,056 \\
\hline 56 & MARYLAND & 66,128 & 113 & GUELPH & 20,131 \\
\hline 57 & MONTREAL & 64,371 & & & \\
\hline
\end{tabular}




\section{RANK ORDER TABLE 3 \\ CuRRENT SERIALs (Totals)}

\begin{tabular}{|c|c|c|c|c|c|}
\hline RANK & INSTITUTION & VALUE & RANK & INSTITUTION & VALUE \\
\hline 1 & MICHIGAN & 118,654 & 58 & SOUTHERN ILLINOIS & 40,490 \\
\hline 2 & COLUMBIA & 117,264 & 59 & BROWN & 40,082 \\
\hline 3 & CALIFORNIA, BERKELEY & 114,860 & 60 & MICHIGAN STATE & 40,082 \\
\hline 4 & HARVARD & 98,988 & 61 & GEORGIA & 39,226 \\
\hline 5 & FLORIDA & 85,169 & 62 & LOUISVILLE & 37,931 \\
\hline 6 & MCGILL & 81,772 & 63 & EMORY & 37,779 \\
\hline 7 & INDIANA & 79,427 & 64 & SUNY-STONY BROOK & 37,497 \\
\hline 8 & CALIFORNIA, LOS ANGELES & 77,509 & 65 & SUNY-BUFFALO & 37,288 \\
\hline 9 & CORNELL & 77,392 & 66 & SASKATCHEWAN & 37,208 \\
\hline 10 & JOHNS HOPKINS & 74,244 & 67 & VIRGINIA TECH & 36,988 \\
\hline 11 & YALE & 73,953 & 68 & CALIFORNIA, SANTA BARBARA & 36,962 \\
\hline 12 & MINNESOTA & 72,573 & 69 & OHIO STATE & 36,813 \\
\hline 13 & VIRGINIA & 71,832 & 70 & PURDUE & 36,296 \\
\hline 14 & PENNSYLVANIA STATE & 71,230 & 71 & MISSOURI & 36,244 \\
\hline 15 & ALBERTA & 69,367 & 72 & VANDERBILT & 36,184 \\
\hline 16 & TORONTO & 68,790 & 73 & GEORGIA TECH & 34,576 \\
\hline 17 & WISCONSIN & 68,560 & 74 & ALABAMA & 34,461 \\
\hline 18 & OKLAHOMA & 63,431 & 75 & ARIZONA & 33,899 \\
\hline 19 & ILLINOIS, URBANA & 63,413 & 76 & HAWAII & 33,291 \\
\hline 20 & OKLAHOMA STATE & 63,116 & 77 & ARIZONA STATE & 33,200 \\
\hline 21 & NEW YORK & 62,537 & 78 & TENNESSEE & 33,184 \\
\hline 22 & WASHINGTON & 62,429 & 79 & MARYLAND & 32,777 \\
\hline 23 & MONTREAL & 62,374 & 80 & CALIFORNIA, SAN DIEGO & 32,088 \\
\hline 24 & LOUISIANA STATE & 60,889 & 81 & KENTUCKY & 31,897 \\
\hline 25 & SOUTHERN CALIFORNIA & 60,718 & 82 & COLORADO STATE & 31,382 \\
\hline 26 & TEXAS TECH & 60,348 & 83 & IOWA STATE & 31,256 \\
\hline 27 & SOUTH CAROLINA & 58,855 & 84 & GEORGETOWN & 31,196 \\
\hline 28 & DUKE & 58,282 & 85 & BRIGHAM YOUNG & 30,895 \\
\hline 29 & CONNECTICUT & 57,270 & 86 & COLORADO & 30,221 \\
\hline 30 & BRITISH COLUMBIA & 56,959 & 87 & WASHINGTON STATE & 30,122 \\
\hline 31 & DARTMOUTH & 55,389 & 88 & CALIFORNIA, RIVERSIDE & 29,941 \\
\hline 32 & NORTH CAROLINA STATE & 54,843 & 89 & TEMPLE & 29,608 \\
\hline 33 & NORTH CAROLINA & 54,591 & 90 & MANITOBA & 28,928 \\
\hline 34 & BOSTON COLLEGE & 53,926 & 91 & LAVAL & 28,417 \\
\hline 35 & NEBRASKA & 53,466 & 92 & ROCHESTER & 26,760 \\
\hline 36 & RUTGERS & 53,302 & 93 & MCMASTER & 26,477 \\
\hline 37 & CALIFORNIA, IRVINE & 53,050 & 94 & GEORGE WASHINGTON & 25,153 \\
\hline 38 & FLORIDA STATE & 51,896 & 95 & NEW MEXICO & 24,323 \\
\hline 39 & IOWA & 51,374 & 96 & SUNY-ALBANY & 23,907 \\
\hline 40 & MIAMI & 51,349 & 97 & WAYNE STATE & 23,693 \\
\hline 41 & ILLINOIS, CHICAGO & 50,325 & 98 & OREGON & 23,186 \\
\hline 42 & PITTSBURGH & 50,232 & 99 & SYRACUSE & 22,865 \\
\hline 43 & KANSAS & 48,037 & 100 & MIT & 22,332 \\
\hline 44 & PENNSYLVANIA & 47,787 & 101 & HOUSTON & 22,193 \\
\hline 45 & CALIFORNIA, DAVIS & 47,008 & 102 & OHIO & 22,081 \\
\hline 46 & TEXAS & 46,880 & 103 & NOTRE DAME & 21,622 \\
\hline 47 & WESTERN ONTARIO & 46,851 & 104 & WATERLOO & 20,524 \\
\hline 48 & TEXAS A\&M & 45,806 & 105 & TULANE & 19,716 \\
\hline 49 & QUEEN'S & 45,363 & 106 & AUBURN & 18,694 \\
\hline 50 & NORTHWESTERN & 45,259 & 107 & CASE WESTERN RESERVE & 18,422 \\
\hline 51 & CHICAGO & 43,390 & 108 & RICE & 15,854 \\
\hline 52 & CINCINNATI & 42,265 & 109 & KENT STATE & 13,726 \\
\hline 53 & PRINCETON & 41,775 & 110 & DELAWARE & 12,532 \\
\hline 54 & WASHINGTON U.-ST. LOUIS & 41,339 & 111 & GUELPH & 11,804 \\
\hline 55 & BOSTON & 40,757 & 112 & HOWARD & 11,333 \\
\hline 56 & MASSACHUSETTS & 40,749 & 113 & YORK & 9,084 \\
\hline 57 & UTAH & 40,607 & & & \\
\hline
\end{tabular}




\section{RANK ORDER TABLE 4 MICROFORM UNITS}

\begin{tabular}{|c|c|c|c|c|c|}
\hline RANK & INSTITUTION & VALUE & RANK & INSTITUTION & VALUE \\
\hline 1 & YALE & $10,123,626$ & 58 & DUKE & $4,215,023$ \\
\hline 2 & HARVARD & $10,008,356$ & 59 & BOSTON COLLEGE & $4,102,631$ \\
\hline 3 & FLORIDA STATE & $9,094,077$ & 60 & PENNSYLVANIA & $4,085,467$ \\
\hline 4 & ILLINOIS, URBANA & $8,508,526$ & 61 & YORK & $4,057,687$ \\
\hline 5 & CORNELL & $8,327,016$ & 62 & ALABAMA & $4,045,077$ \\
\hline 6 & FLORIDA & $7,752,132$ & 63 & WESTERN ONTARIO & $3,991,614$ \\
\hline 7 & ARIZONA STATE & $7,712,343$ & 64 & ILLINOIS, CHICAGO & $3,948,866$ \\
\hline 8 & WASHINGTON & $7,680,082$ & 65 & GEORGETOWN & $3,945,820$ \\
\hline 9 & PITTSBURGH & $7,543,846$ & 66 & MIAMI & $3,941,072$ \\
\hline 10 & MISSOURI & $7,524,227$ & 67 & QUEEN'S & $3,885,140$ \\
\hline 11 & SYRACUSE & $7,401,625$ & 68 & NOTRE DAME & $3,871,875$ \\
\hline 12 & IOWA & $7,189,464$ & 69 & WAYNE STATE & $3,866,924$ \\
\hline 13 & MINNESOTA & $6,900,505$ & 70 & BRIGHAM YOUNG & $3,837,384$ \\
\hline 14 & CALIFORNIA, BERKELEY & $6,891,881$ & 71 & SUNY-STONY BROOK & $3,834,398$ \\
\hline 15 & COLORADO & $6,843,252$ & 72 & HOWARD & $3,776,923$ \\
\hline 16 & LOUISIANA STATE & $6,733,022$ & 73 & CALIFORNIA, SANTA BARBARA & $3,771,751$ \\
\hline 17 & MICHIGAN STATE & $6,676,334$ & 74 & ALBERTA & $3,726,833$ \\
\hline 18 & GEORGIA & $6,580,560$ & 75 & KANSAS & $3,623,655$ \\
\hline 19 & TEXAS & $6,570,665$ & 76 & UTAH & $3,619,914$ \\
\hline 20 & PRINCETON & $6,521,389$ & 77 & HAWAII & $3,502,277$ \\
\hline 21 & KENTUCKY & $6,388,872$ & 78 & IOWA STATE & $3,491,104$ \\
\hline 22 & NEW YORK & $6,372,947$ & 79 & DELAWARE & $3,428,291$ \\
\hline 23 & VIRGINIA TECH & $6,318,427$ & 80 & WASHINGTON U.-ST. LOUIS & $3,392,968$ \\
\hline 24 & WISCONSIN & $6,277,775$ & 81 & CINCINNATI & $3,346,254$ \\
\hline 25 & ARIZONA & $6,247,714$ & 82 & $\mathrm{OHIO}$ & $3,278,156$ \\
\hline 26 & COLUMBIA & $6,245,400$ & 83 & CALIFORNIA, SAN DIEGO & $3,260,769$ \\
\hline 27 & SOUTHERN CALIFORNIA & $6,221,738$ & 84 & TEMPLE & $3,214,480$ \\
\hline 28 & CALIFORNIA, LOS ANGELES & $6,181,047$ & 85 & RICE & $3,204,245$ \\
\hline 29 & EMORY & $6,157,328$ & 86 & PURDUE & $3,099,407$ \\
\hline 30 & RUTGERS & $5,935,929$ & 87 & SASKATCHEWAN & $3,084,671$ \\
\hline 31 & HOUSTON & $5,917,649$ & 88 & CHICAGO & $3,044,663$ \\
\hline 32 & OHIO STATE & $5,912,179$ & 89 & VANDERBILT & $3,043,451$ \\
\hline 33 & MARYLAND & $5,771,015$ & 90 & OREGON & $2,920,159$ \\
\hline 34 & TEXAS A\&M & $5,543,084$ & 91 & GEORGE WASHINGTON & $2,900,748$ \\
\hline 35 & VIRGINIA & $5,474,181$ & 92 & CALIFORNIA, IRVINE & $2,889,312$ \\
\hline 36 & NORTH CAROLINA STATE & $5,443,193$ & 93 & SUNY-ALBANY & $2,876,879$ \\
\hline 37 & SUNY-BUFFALO & $5,442,977$ & 94 & AUBURN & $2,690,621$ \\
\hline 38 & TORONTO & $5,425,323$ & 95 & TEXAS TECH & $2,630,207$ \\
\hline 39 & PENNSYLVANIA STATE & $5,294,473$ & 96 & DARTMOUTH & $2,584,144$ \\
\hline 40 & INDIANA & $5,292,522$ & 97 & MASSACHUSETTS & $2,580,684$ \\
\hline 41 & SOUTH CAROLINA & $5,262,414$ & 98 & CASE WESTERN RESERVE & $2,565,701$ \\
\hline 42 & BRITISH COLUMBIA & $5,225,508$ & 99 & MIT & $2,401,703$ \\
\hline 43 & ROCHESTER & $5,103,919$ & 100 & GUELPH & $2,326,827$ \\
\hline 44 & NORTH CAROLINA & $5,023,954$ & 101 & LOUISVILLE & $2,201,300$ \\
\hline 45 & NEW MEXICO & $4,708,918$ & 102 & CALIFORNIA, RIVERSIDE & $2,182,650$ \\
\hline 46 & NEBRASKA & $4,630,935$ & 103 & BROWN & $1,947,043$ \\
\hline 47 & BOSTON & $4,612,586$ & 104 & MCGILL & $1,826,291$ \\
\hline 48 & SOUTHERN ILLINOIS & $4,588,909$ & 105 & WATERLOO & $1,708,666$ \\
\hline 49 & GEORGIA TECH & $4,564,518$ & 106 & MCMASTER & $1,612,607$ \\
\hline 50 & OKLAHOMA STATE & $4,502,389$ & 107 & MONTREAL & $1,533,723$ \\
\hline 51 & NORTHWESTERN & $4,458,193$ & 108 & MANITOBA & $1,469,948$ \\
\hline 52 & CONNECTICUT & $4,404,479$ & 109 & LAVAL & $1,330,408$ \\
\hline 53 & JOHNS HOPKINS & $4,388,145$ & 110 & TULANE & $1,265,125$ \\
\hline 54 & OKLAHOMA & $4,349,115$ & 111 & COLORADO STATE & $1,197,144$ \\
\hline 55 & WASHINGTON STATE & $4,229,240$ & 112 & KENT STATE & UA \\
\hline 56 & TENNESSEE & $4,225,398$ & 113 & MICHIGAN & UA \\
\hline 57 & CALIFORNIA, DAVIS & $4,223,277$ & & & \\
\hline
\end{tabular}

UA - Unavailable 


\section{Rank Order Table 5}

GOVERNMENT DOCUMENTS

\begin{tabular}{|c|c|c|c|c|c|}
\hline RANK & INSTITUTION & VALUE & RANK & INSTITUTION & VALUE \\
\hline 1 & RUTGERS & $2,663,310$ & 58 & RICE & 55,896 \\
\hline 2 & MINNESOTA & $2,637,739$ & 59 & CINCINNATI & 34,353 \\
\hline 3 & MISSOURI & $1,663,855$ & 60 & CASE WESTERN RESERVE & 24,717 \\
\hline 4 & NORTH CAROLINA & $1,625,932$ & 61 & OKLAHOMA & 24,065 \\
\hline 5 & FLORIDA & $1,551,630$ & 62 & NORTHWESTERN & 7,808 \\
\hline 6 & DUKE & $1,248,520$ & 63 & TENNESSEE & 5,297 \\
\hline 7 & KENTUCKY & $1,107,296$ & 64 & TEXAS TECH & 4,222 \\
\hline 8 & QUEEN'S & $1,106,135$ & 65 & HOWARD & 2,145 \\
\hline 9 & GEORGIA & $1,060,065$ & 66 & OHIO STATE & 2,080 \\
\hline 10 & COLORADO & 966,668 & 67 & TEMPLE & 410 \\
\hline 11 & ALABAMA & 934,159 & 68 & JOHNS HOPKINS & 80 \\
\hline 12 & SOUTH CAROLINA & 923,489 & 69 & ALBERTA & 0 \\
\hline 13 & UTAH & 885,109 & 70 & ARIZONA & 0 \\
\hline 14 & FLORIDA STATE & 855,373 & 71 & BROWN & 0 \\
\hline 15 & WISCONSIN & 811,086 & 72 & CALIFORNIA, SAN DIEGO & 0 \\
\hline 16 & ARIZONA STATE & 800,678 & 73 & DARTMOUTH & 0 \\
\hline 17 & ILLINOIS, CHICAGO & 776,943 & 74 & GEORGE WASHINGTON & 0 \\
\hline 18 & KANSAS & 734,016 & 75 & GEORGIA TECH & 0 \\
\hline 19 & HOUSTON & 680,971 & 76 & GUELPH & 0 \\
\hline 20 & VIRGINIA & 676,242 & 77 & HAWAII & 0 \\
\hline 21 & MCGILL & 672,147 & 78 & ILLINOIS, URBANA & 0 \\
\hline 22 & IOWA & 665,618 & 79 & IOWA STATE & 0 \\
\hline 23 & NEW MEXICO & 655,929 & 80 & KENT STATE & 0 \\
\hline 24 & LOUISIANA STATE & 601,036 & 81 & LAVAL & 0 \\
\hline 25 & NEBRASKA & 552,672 & 82 & LOUISVILLE & 0 \\
\hline 26 & CALIFORNIA, BERKELEY & 508,081 & 83 & MCMASTER & 0 \\
\hline 27 & PURDUE & 489,215 & 84 & MIAMI & 0 \\
\hline 28 & NEW YORK & 479,462 & 85 & MICHIGAN STATE & 0 \\
\hline 29 & OREGON & 467,921 & 86 & MONTREAL & 0 \\
\hline 30 & DELAWARE & 448,183 & 87 & NORTH CAROLINA STATE & 0 \\
\hline 31 & ROCHESTER & 443,509 & 88 & PENNSYLVANIA STATE & 0 \\
\hline 32 & CALIFORNIA, SANTA BARBARA & 419,256 & 89 & SUNY-ALBANY & 0 \\
\hline 33 & BRIGHAM YOUNG & 418,406 & 90 & TEXAS & 0 \\
\hline 34 & SASKATCHEWAN & 414,542 & 91 & WASHINGTON & 0 \\
\hline 35 & VIRGINIA TECH & 399,525 & 92 & WASHINGTON STATE & 0 \\
\hline 36 & WAYNE STATE & 388,182 & 93 & WASHINGTON U.-ST. LOUIS & 0 \\
\hline 37 & SUNY-STONY BROOK & 339,423 & 94 & WATERLOO & 0 \\
\hline 38 & INDIANA & 314,450 & 95 & WESTERN ONTARIO & 0 \\
\hline 39 & SOUTHERN ILLINOIS & 313,173 & 96 & YALE & 0 \\
\hline 40 & SYRACUSE & 306,768 & 97 & BOSTON & UA \\
\hline 41 & EMORY & 303,566 & 98 & BRITISH COLUMBIA & UA \\
\hline 42 & CALIFORNIA, RIVERSIDE & 297,963 & 99 & CHICAGO & UA \\
\hline 43 & SOUTHERN CALIFORNIA & 268,670 & 100 & CONNECTICUT & UA \\
\hline 44 & VANDERBILT & 257,762 & 101 & CORNELL & UA \\
\hline 45 & AUBURN & 241,622 & 102 & GEORGETOWN & UA \\
\hline 46 & COLORADO STATE & 227,953 & 103 & HARVARD & UA \\
\hline 47 & MANITOBA & 224,980 & 104 & MASSACHUSETTS & UA \\
\hline 48 & BOSTON COLLEGE & 220,915 & 105 & MIT & UA \\
\hline 49 & CALIFORNIA, IRVINE & 200,063 & 106 & MICHIGAN & UA \\
\hline 50 & COLUMBIA & 194,156 & 107 & NOTRE DAME & UA \\
\hline 51 & MARYLAND & 188,990 & 108 & $\mathrm{OHIO}$ & UA \\
\hline 52 & OKLAHOMA STATE & 158,729 & 109 & PENNSYLVANIA & UA \\
\hline 53 & TULANE & 145,911 & 110 & PITTSBURGH & UA \\
\hline 54 & PRINCETON & 120,460 & 111 & SUNY-BUFFALO & UA \\
\hline 55 & CALIFORNIA, DAVIS & 74,849 & 112 & TORONTO & UA \\
\hline 56 & CALIFORNIA, LOS ANGELES & 69,577 & 113 & YORK & UA \\
\hline 57 & TEXAS A\&M & 65,519 & & & \\
\hline
\end{tabular}

UA - Unavailable 
RANK ORDER TABLE 6

TOTAL LIBRARY MATERIALS EXPENDITURES

\begin{tabular}{|c|c|c|c|c|c|}
\hline RANK & INSTITUTION & VALUE & RANK & INSTITUTION & VALUE \\
\hline 1 & YALE & $33,913,555$ & 58 & NORTH CAROLINA STATE & $9,002,518$ \\
\hline 2 & HARVARD & $28,138,927$ & 59 & IOWA STATE & $8,891,487$ \\
\hline 3 & TORONTO & $21,450,043$ & 60 & LAVAL & $8,801,485$ \\
\hline 4 & COLUMBIA & $21,017,791$ & 61 & TEMPLE & $8,747,157$ \\
\hline 5 & MICHIGAN & $20,669,495$ & 62 & BOSTON & $8,707,614$ \\
\hline 6 & PENNSYLVANIA STATE & $18,088,877$ & 63 & MARYLAND & $8,698,140$ \\
\hline 7 & CALIFORNIA, BERKELEY & $17,453,180$ & 64 & CALIFORNIA, DAVIS & $8,659,069$ \\
\hline 8 & PRINCETON & $16,754,840$ & 65 & YORK & $8,384,544$ \\
\hline 9 & CHICAGO & $16,462,379$ & 66 & BROWN & $8,285,394$ \\
\hline 10 & NEW YORK & $16,139,134$ & 67 & ILLINOIS, CHICAGO & $8,166,623$ \\
\hline 11 & TEXAS & $16,035,009$ & 68 & HOUSTON & $8,117,193$ \\
\hline 12 & CALIFORNIA, LOS ANGELES & $15,158,149$ & 69 & KANSAS & $8,053,844$ \\
\hline 13 & ALBERTA & $14,590,484$ & 70 & BOSTON COLLEGE & $8,009,522$ \\
\hline 14 & CORNELL & $14,381,579$ & 71 & DELAWARE & $8,005,686$ \\
\hline 15 & MINNESOTA & $14,157,172$ & 72 & MIT & $7,954,606$ \\
\hline 16 & TEXAS A\&M & $14,099,481$ & 73 & CALIFORNIA, SAN DIEGO & $7,922,917$ \\
\hline 17 & EMORY & $14,083,609$ & 74 & QUEEN'S & $7,881,622$ \\
\hline 18 & NORTH CAROLINA & $14,039,479$ & 75 & CONNECTICUT & $7,854,879$ \\
\hline 19 & DUKE & $13,961,002$ & 76 & CALIFORNIA, IRVINE & $7,814,726$ \\
\hline 20 & INDIANA & $13,907,828$ & 77 & WAYNE STATE & $7,811,606$ \\
\hline 21 & PENNSYLVANIA & $13,749,248$ & 78 & SUNY-BUFFALO & $7,765,537$ \\
\hline 22 & JOHNS HOPKINS & $13,638,688$ & 79 & SASKATCHEWAN & $7,763,830$ \\
\hline 23 & PITTSBURGH & $13,637,310$ & 80 & DARTMOUTH & $7,748,473$ \\
\hline 24 & BRITISH COLUMBIA & $13,523,834$ & 81 & TULANE & $7,675,475$ \\
\hline 25 & SOUTHERN CALIFORNIA & $12,962,417$ & 82 & FLORIDA STATE & $7,650,054$ \\
\hline 26 & IOWA & $12,546,477$ & 83 & HAWAII & $7,611,078$ \\
\hline 27 & WASHINGTON & $12,542,511$ & 84 & UTAH & $7,489,866$ \\
\hline 28 & OHIO STATE & $12,205,939$ & 85 & VIRGINIA TECH & $7,371,492$ \\
\hline 29 & NORTHWESTERN & $12,165,603$ & 86 & SOUTHERN ILLINOIS & $7,117,514$ \\
\hline 30 & OKLAHOMA & $12,118,386$ & 87 & SOUTH CAROLINA & $7,066,564$ \\
\hline 31 & ILLINOIS, URBANA & $12,043,672$ & 88 & NEBRASKA & $7,008,885$ \\
\hline 32 & MIAMI & $12,025,519$ & 89 & ALABAMA & $6,870,657$ \\
\hline 33 & ARIZONA & $11,662,200$ & 90 & MCMASTER & $6,829,286$ \\
\hline 34 & WISCONSIN & $11,582,710$ & 91 & OKLAHOMA STATE & $6,783,316$ \\
\hline 35 & MCGILL & $11,549,953$ & 92 & MANITOBA & $6,758,968$ \\
\hline 36 & TENNESSEE & $11,547,387$ & 93 & ROCHESTER & $6,715,587$ \\
\hline 37 & ARIZONA STATE & $11,474,442$ & 94 & LOUISIANA STATE & $6,675,849$ \\
\hline 38 & GEORGIA & $11,217,811$ & 95 & SUNY-STONY BROOK & $6,590,728$ \\
\hline 39 & PURDUE & $10,974,071$ & 96 & MISSOURI & $6,462,575$ \\
\hline 40 & FLORIDA & $10,821,088$ & 97 & CASE WESTERN RESERVE & $6,304,139$ \\
\hline 41 & GEORGETOWN & $10,783,633$ & 98 & SYRACUSE & $6,300,160$ \\
\hline 42 & WASHINGTON U.-ST. LOUIS & $10,646,463$ & 99 & COLORADO STATE & $6,265,271$ \\
\hline 43 & RUTGERS & $10,610,826$ & 100 & WATERLOO & $6,180,735$ \\
\hline 44 & VANDERBILT & $10,577,813$ & 101 & CALIFORNIA, RIVERSIDE & $6,109,872$ \\
\hline 45 & MONTREAL & $10,361,290$ & 102 & WASHINGTON STATE & $5,628,649$ \\
\hline 46 & CINCINNATI & $10,209,066$ & 103 & MASSACHUSETTS & $5,579,248$ \\
\hline 47 & VIRGINIA & $10,039,183$ & 104 & AUBURN & $5,543,726$ \\
\hline 48 & MICHIGAN STATE & $9,949,499$ & 105 & OHIO & $5,327,277$ \\
\hline 49 & RICE & $9,764,416$ & 106 & GEORGIA TECH & $5,306,847$ \\
\hline 50 & NOTRE DAME & $9,540,297$ & 107 & NEW MEXICO & $5,305,708$ \\
\hline 51 & TEXAS TECH & $9,503,529$ & 108 & CALIFORNIA, SANTA BARBARA & $5,236,330$ \\
\hline 52 & KENTUCKY & $9,463,513$ & 109 & SUNY-ALBANY & $5,231,629$ \\
\hline 53 & GEORGE WASHINGTON & $9,457,211$ & 110 & OREGON & $5,135,978$ \\
\hline 54 & COLORADO & $9,315,863$ & 111 & GUELPH & $5,039,056$ \\
\hline 55 & BRIGHAM YOUNG & $9,271,776$ & 112 & KENT STATE & $3,603,707$ \\
\hline 56 & WESTERN ONTARIO & $9,174,734$ & 113 & HOWARD & $3,411,656$ \\
\hline 57 & LOUISVILLE & $9,156,974$ & & & \\
\hline
\end{tabular}


RANK ORDER TABLE 7

TOTAL SALARIES \& WAGES EXPENDITURES

\begin{tabular}{|c|c|c|c|c|c|}
\hline RANK & INSTITUTION & VALUE & RANK & INSTITUTION & VALUE \\
\hline 1 & HARVARD & $58,047,311$ & 58 & TENNESSEE & $9,434,712$ \\
\hline 2 & CALIFORNIA, BERKELEY & $31,968,998$ & 59 & MANITOBA & $9,402,859$ \\
\hline 3 & YALE & $31,304,471$ & 60 & GEORGE WASHINGTON & $9,148,256$ \\
\hline 4 & TORONTO & $29,821,592$ & 61 & VANDERBILT & $9,003,218$ \\
\hline 5 & COLUMBIA & $29,057,396$ & 62 & WAYNE STATE & $8,812,470$ \\
\hline 6 & CALIFORNIA, LOS ANGELES & $26,270,989$ & 63 & CALIFORNIA, IRVINE & $8,663,498$ \\
\hline 7 & PENNSYLVANIA STATE & $23,496,010$ & 64 & BROWN & $8,648,110$ \\
\hline 8 & MICHIGAN & $23,203,751$ & 65 & BOSTON & $8,523,627$ \\
\hline 9 & WISCONSIN & $22,380,731$ & 66 & MIAMI & $8,446,548$ \\
\hline 10 & CORNELL & $21,196,729$ & 67 & LAVAL & $8,426,864$ \\
\hline 11 & WASHINGTON & $20,566,966$ & 68 & CALIFORNIA, SANTA BARBARA & $8,332,750$ \\
\hline 12 & VIRGINIA & $20,204,507$ & 69 & COLORADO & $8,251,807$ \\
\hline 13 & TEXAS & $19,810,313$ & 70 & SYRACUSE & $8,240,340$ \\
\hline 14 & ILLINOIS, URBANA & $19,151,325$ & 71 & TEXAS TECH & $8,231,498$ \\
\hline 15 & RUTGERS & $18,257,664$ & 72 & ROCHESTER & $8,231,005$ \\
\hline 16 & NEW YORK & $18,185,623$ & 73 & BOSTON COLLEGE & $8,227,190$ \\
\hline 17 & PRINCETON & $17,639,339$ & 74 & HAWAII & $8,126,908$ \\
\hline 18 & MINNESOTA & $17,469,535$ & 75 & KENTUCKY & $8,008,601$ \\
\hline 19 & MONTREAL & $16,443,397$ & 76 & DARTMOUTH & $7,940,926$ \\
\hline 20 & NORTH CAROLINA & $16,202,923$ & 77 & SOUTH CAROLINA & $7,837,177$ \\
\hline 21 & PENNSYLVANIA & $15,214,681$ & 78 & ILLINOIS, CHICAGO & $7,793,319$ \\
\hline 22 & OHIO STATE & $15,154,611$ & 79 & DELAWARE & $7,199,440$ \\
\hline 23 & DUKE & $14,598,282$ & 80 & TEMPLE & $7,178,583$ \\
\hline 24 & SOUTHERN CALIFORNIA & $14,534,193$ & 81 & MASSACHUSETTS & $7,176,049$ \\
\hline 25 & INDIANA & $14,312,985$ & 82 & WESTERN ONTARIO & $6,972,741$ \\
\hline 26 & BRITISH COLUMBIA & $13,865,835$ & 83 & IOWA STATE & $6,947,368$ \\
\hline 27 & ALBERTA & $13,809,237$ & 84 & OREGON & $6,921,185$ \\
\hline 28 & CONNECTICUT & $13,693,118$ & 85 & FLORIDA STATE & $6,903,278$ \\
\hline 29 & CALIFORNIA, SAN DIEGO & $13,570,569$ & 86 & NEBRASKA & $6,640,382$ \\
\hline 30 & WASHINGTON U.-ST. LOUIS & $12,923,180$ & 87 & SOUTHERN ILLINOIS & $6,542,641$ \\
\hline 31 & EMORY & $12,542,750$ & 88 & QUEEN'S & $6,383,917$ \\
\hline 32 & FLORIDA & $12,339,134$ & 89 & SUNY-STONY BROOK & $6,306,810$ \\
\hline 33 & NORTHWESTERN & $12,067,646$ & 90 & WASHINGTON STATE & $6,268,694$ \\
\hline 34 & PITTSBURGH & $11,869,412$ & 91 & CALIFORNIA, RIVERSIDE & $6,253,811$ \\
\hline 35 & ARIZONA & $11,755,093$ & 92 & SUNY-ALBANY & $6,111,639$ \\
\hline 36 & CHICAGO & $11,635,404$ & 93 & MISSOURI & $6,051,328$ \\
\hline 37 & MIT & $11,574,719$ & 94 & WATERLOO & $6,000,777$ \\
\hline 38 & ARIZONA STATE & $11,520,417$ & 95 & LOUISVILLE & $5,982,693$ \\
\hline 39 & IOWA & $11,334,699$ & 96 & OKLAHOMA & $5,917,957$ \\
\hline 40 & MARYLAND & $11,195,093$ & 97 & HOUSTON & $5,910,147$ \\
\hline 41 & UTAH & $11,182,891$ & 98 & OHIO & $5,901,095$ \\
\hline 42 & BRIGHAM YOUNG & $11,177,134$ & 99 & SASKATCHEWAN & $5,878,972$ \\
\hline 43 & TEXAS A\&M & $11,077,467$ & 100 & CASE WESTERN RESERVE & $5,846,752$ \\
\hline 44 & MICHIGAN STATE & $10,896,612$ & 101 & ALABAMA & $5,797,704$ \\
\hline 45 & NEW MEXICO & $10,876,975$ & 102 & MCMASTER & $5,631,285$ \\
\hline 46 & MCGILL & $10,501,564$ & 103 & TULANE & $5,620,318$ \\
\hline 47 & GEORGETOWN & $10,422,635$ & 104 & COLORADO STATE & $5,398,029$ \\
\hline 48 & JOHNS HOPKINS & $10,147,962$ & 105 & OKLAHOMA STATE & $5,290,295$ \\
\hline 49 & CINCINNATI & $10,071,807$ & 106 & VIRGINIA TECH & $5,232,155$ \\
\hline 50 & NORTH CAROLINA STATE & $10,033,422$ & 107 & GUELPH & $5,209,953$ \\
\hline 51 & SUNY-BUFFALO & $9,964,759$ & 108 & HOWARD & $5,119,471$ \\
\hline 52 & PURDUE & $9,924,575$ & 109 & LOUISIANA STATE & $5,097,544$ \\
\hline 53 & KANSAS & $9,771,011$ & 110 & RICE & $4,936,852$ \\
\hline 54 & CALIFORNIA, DAVIS & $9,755,811$ & 111 & GEORGIA TECH & $4,796,837$ \\
\hline 55 & NOTRE DAME & $9,539,578$ & 112 & AUBURN & $4,680,882$ \\
\hline 56 & GEORGIA & $9,487,337$ & 113 & KENT STATE & $4,440,988$ \\
\hline 57 & YORK & $9,444,855$ & & & \\
\hline
\end{tabular}

UA - Unavailable 


\section{RANK ORder TABLE 8}

OTHER OPERATING EXPENDITURES

\begin{tabular}{|c|c|c|c|c|c|}
\hline RANK & INSTITUTION & VAlue & RANK & INSTITUTION & VALUE \\
\hline 1 & HARVARD & $18,605,497$ & 58 & GEORGETOWN & $2,440,663$ \\
\hline 2 & YALE & $9,131,178$ & 59 & OHIO & $2,356,964$ \\
\hline 3 & CALIFORNIA, LOS ANGELES & $9,015,043$ & 60 & ROCHESTER & $2,336,792$ \\
\hline 4 & WASHINGTON U.-ST. LOUIS & $7,786,020$ & 61 & NEBRASKA & $2,334,020$ \\
\hline 5 & NORTH CAROLINA STATE & $7,483,903$ & 62 & AUBURN & $2,283,891$ \\
\hline 6 & WISCONSIN & $6,607,997$ & 63 & CALIFORNIA, RIVERSIDE & $2,278,507$ \\
\hline 7 & PENNSYLVANIA STATE & $6,567,041$ & 64 & RUTGERS & $2,244,119$ \\
\hline 8 & NEW YORK & $6,424,257$ & 65 & CONNECTICUT & $2,171,752$ \\
\hline 9 & CORNELL & $6,404,870$ & 66 & ALABAMA & $2,120,819$ \\
\hline 10 & MINNESOTA & $6,357,109$ & 67 & BOSTON & $2,113,448$ \\
\hline 11 & COLUMBIA & $6,223,006$ & 68 & ALBERTA & $2,063,189$ \\
\hline 12 & CALIFORNIA, BERKELEY & $6,021,132$ & 69 & GEORGIA & $2,046,444$ \\
\hline 13 & TORONTO & $5,940,464$ & 70 & CALIFORNIA, DAVIS & $2,031,184$ \\
\hline 14 & TEXAS & $5,572,367$ & 71 & KENTUCKY & $1,934,664$ \\
\hline 15 & WASHINGTON & $5,486,661$ & 72 & QUEEN'S & $1,907,213$ \\
\hline 16 & SOUTH CAROLINA & $5,418,559$ & 73 & NORTHWESTERN & $1,883,240$ \\
\hline 17 & OHIO STATE & $5,255,845$ & 74 & MISSOURI & $1,879,906$ \\
\hline 18 & CALIFORNIA, SANTA BARBARA & $4,851,553$ & 75 & ILLINOIS, CHICAGO & $1,861,703$ \\
\hline 19 & DUKE & $4,818,896$ & 76 & MASSACHUSETTS & $1,836,714$ \\
\hline 20 & ARIZONA & $4,818,457$ & 77 & TEMPLE & $1,835,712$ \\
\hline 21 & PENNSYLVANIA & $4,679,437$ & 78 & BROWN & $1,797,823$ \\
\hline 22 & ILLINOIS, URBANA & $4,639,760$ & 79 & OKLAHOMA STATE & $1,792,118$ \\
\hline 23 & CALIFORNIA, SAN DIEGO & $4,529,147$ & 80 & TENNESSEE & $1,756,502$ \\
\hline 24 & UTAH & $4,469,965$ & 81 & DARTMOUTH & $1,693,392$ \\
\hline 25 & VIRGINIA & $4,448,759$ & 82 & COLORADO STATE & $1,670,566$ \\
\hline 26 & WAYNE STATE & $4,370,981$ & 83 & OKLAHOMA & $1,648,347$ \\
\hline 27 & SOUTHERN CALIFORNIA & $4,264,218$ & 84 & DELAWARE & $1,647,113$ \\
\hline 28 & JOHNS HOPKINS & $4,253,334$ & 85 & MIT & $1,641,302$ \\
\hline 29 & PRINCETON & $4,220,797$ & 86 & SUNY-BUFFALO & $1,628,145$ \\
\hline 30 & TEXAS A\&M & $4,200,120$ & 87 & IOWA & $1,601,683$ \\
\hline 31 & FLORIDA & $4,097,979$ & 88 & OREGON & $1,580,709$ \\
\hline 32 & NORTH CAROLINA & $4,024,002$ & 89 & WASHINGTON STATE & $1,573,838$ \\
\hline 33 & EMORY & $4,007,780$ & 90 & VIRGINIA TECH & $1,436,106$ \\
\hline 34 & MCGILL & $3,633,371$ & 91 & HAWAII & $1,410,600$ \\
\hline 35 & INDIANA & $3,629,712$ & 92 & COLORADO & $1,380,087$ \\
\hline 36 & MARYLAND & $3,565,022$ & 93 & CALIFORNIA, IRVINE & $1,297,937$ \\
\hline 37 & TEXAS TECH & $3,489,492$ & 94 & MONTREAL & $1,268,900$ \\
\hline 38 & VANDERBILT & $3,308,978$ & 95 & HOWARD & $1,263,805$ \\
\hline 39 & BRIGHAM YOUNG & $3,259,004$ & 96 & SYRACUSE & $1,235,583$ \\
\hline 40 & LOUISVILLE & $3,253,551$ & 97 & GEORGIA TECH & $1,232,259$ \\
\hline 41 & CHICAGO & $3,223,018$ & 98 & RICE & $1,146,717$ \\
\hline 42 & PITTSBURGH & $3,198,640$ & 99 & GUELPH & $1,136,966$ \\
\hline 43 & NEW MEXICO & $3,116,758$ & 100 & SUNY-STONY BROOK & $1,133,427$ \\
\hline 44 & MICHIGAN STATE & $3,047,831$ & 101 & YORK & $1,129,115$ \\
\hline 45 & PURDUE & $3,014,032$ & 102 & SASKATCHEWAN & $1,114,615$ \\
\hline 46 & GEORGE WASHINGTON & $2,988,742$ & 103 & BOSTON COLLEGE & $1,106,872$ \\
\hline 47 & MANITOBA & $2,940,720$ & 104 & LOUISIANA STATE & $1,045,925$ \\
\hline 48 & KANSAS & $2,887,628$ & 105 & MCMASTER & $1,040,193$ \\
\hline 49 & HOUSTON & $2,877,961$ & 106 & IOWA STATE & 945,544 \\
\hline 50 & BRITISH COLUMBIA & $2,859,571$ & 107 & TULANE & 929,771 \\
\hline 51 & MIAMI & $2,717,564$ & 108 & SOUTHERN ILLINOIS & 825,601 \\
\hline 52 & KENT STATE & $2,708,329$ & 109 & WESTERN ONTARIO & 751,520 \\
\hline 53 & CASE WESTERN RESERVE & $2,597,127$ & 110 & SUNY-ALBANY & 613,972 \\
\hline 54 & WATERLOO & $2,593,655$ & 111 & FLORIDA STATE & 460,142 \\
\hline 55 & CINCINNATI & $2,552,039$ & 112 & LAVAL & 419,986 \\
\hline 56 & ARIZONA STATE & $2,528,302$ & 113 & MICHIGAN & UA \\
\hline 57 & NOTRE DAME & $2,524,825$ & & & \\
\hline
\end{tabular}

UA - Unavailable 


\section{RANK ORDER TABLE 9 \\ TOTAL LIBRARY EXPENDITURES}

\begin{tabular}{|c|c|c|c|c|c|}
\hline RANK & INSTITUTION & VALUe & RANK & INSTITUTION & VALUE \\
\hline 1 & HARVARD & $105,809,085$ & 58 & WAYNE STATE & $21,065,002$ \\
\hline 2 & YALE & $74,938,119$ & 59 & KANSAS & $20,832,470$ \\
\hline 3 & TORONTO & $57,610,752$ & 60 & CALIFORNIA, DAVIS & $20,609,936$ \\
\hline 4 & COLUMBIA & $56,938,255$ & 61 & SOUTH CAROLINA & $20,422,352$ \\
\hline 5 & CALIFORNIA, BERKELEY & $56,186,972$ & 62 & OKLAHOMA & $19,856,352$ \\
\hline 6 & CALIFORNIA, LOS ANGELES & $50,919,689$ & 63 & KENTUCKY & $19,569,146$ \\
\hline 7 & MICHIGAN & $49,053,402$ & 64 & BOSTON & $19,484,243$ \\
\hline 8 & PENNSYLVANIA STATE & $48,580,052$ & 65 & SUNY-BUFFALO & $19,453,788$ \\
\hline 9 & CORNELL & $42,156,480$ & 66 & NEW MEXICO & $19,398,044$ \\
\hline 10 & TEXAS & $41,585,820$ & 67 & MANITOBA & $19,234,599$ \\
\hline 11 & NEW YORK & $41,003,612$ & 68 & YORK & $19,099,174$ \\
\hline 12 & WISCONSIN & $40,800,267$ & 69 & COLORADO & $19,014,310$ \\
\hline 13 & PRINCETON & $39,143,966$ & 70 & BROWN & $18,945,391$ \\
\hline 14 & WASHINGTON & $38,841,830$ & 71 & CALIFORNIA, SANTA BARBARA & $18,580,864$ \\
\hline 15 & MINNESOTA & $38,321,667$ & 72 & LOUISVILLE & $18,493,616$ \\
\hline 16 & ILLINOIS, URBANA & $36,102,613$ & 73 & ILLINOIS, CHICAGO & $17,887,357$ \\
\hline 17 & VIRGINIA & $34,775,087$ & 74 & TEMPLE & $17,873,359$ \\
\hline 18 & NORTH CAROLINA & $34,488,601$ & 75 & CALIFORNIA, IRVINE & $17,845,419$ \\
\hline 19 & PENNSYLVANIA & $33,897,604$ & 76 & LAVAL & $17,695,209$ \\
\hline 20 & DUKE & $33,531,731$ & 77 & DARTMOUTH & $17,497,174$ \\
\hline 21 & OHIO STATE & $32,966,111$ & 78 & BOSTON COLLEGE & $17,481,965$ \\
\hline 22 & INDIANA & $32,143,391$ & 79 & ROCHESTER & $17,412,084$ \\
\hline 23 & SOUTHERN CALIFORNIA & $31,893,689$ & 80 & HAWAII & $17,306,904$ \\
\hline 24 & CHICAGO & $31,671,892$ & 81 & WESTERN ONTARIO & $17,007,710$ \\
\hline 25 & WASHINGTON U.-ST. LOUIS & $31,466,476$ & 82 & HOUSTON & $17,000,792$ \\
\hline 26 & RUTGERS & $31,253,603$ & 83 & IOWA STATE & $16,973,524$ \\
\hline 27 & EMORY & $30,765,589$ & 84 & DELAWARE & $16,937,444$ \\
\hline 28 & ALBERTA & $30,588,230$ & 85 & QUEEN'S & $16,259,032$ \\
\hline 29 & BRITISH COLUMBIA & $30,420,698$ & 86 & NEBRASKA & $16,138,697$ \\
\hline 30 & TEXAS A\&M & $29,511,695$ & 87 & RICE & $15,909,567$ \\
\hline 31 & PITTSBURGH & $28,927,212$ & 88 & SYRACUSE & $15,836,698$ \\
\hline 32 & ARIZONA & $28,333,910$ & 89 & FLORIDA STATE & $15,083,949$ \\
\hline 33 & MONTREAL & $28,288,912$ & 90 & CALIFORNIA, RIVERSIDE & $14,888,349$ \\
\hline 34 & JOHNS HOPKINS & $28,123,663$ & 91 & ALABAMA & $14,868,525$ \\
\hline 35 & FLORIDA & $27,435,482$ & 92 & CASE WESTERN RESERVE & $14,861,753$ \\
\hline 36 & NORTH CAROLINA STATE & $26,714,162$ & 93 & WATERLOO & $14,838,150$ \\
\hline 37 & NORTHWESTERN & $26,335,228$ & 94 & SASKATCHEWAN & $14,829,710$ \\
\hline 38 & CALIFORNIA, SAN DIEGO & $26,298,111$ & 95 & MASSACHUSETTS & $14,680,447$ \\
\hline 39 & MCGILL & $25,882,656$ & 96 & SOUTHERN ILLINOIS & $14,619,649$ \\
\hline 40 & ARIZONA STATE & $25,677,775$ & 97 & MISSOURI & $14,555,302$ \\
\hline 41 & IOWA & $25,655,780$ & 98 & TULANE & $14,261,505$ \\
\hline 42 & MICHIGAN STATE & $24,109,074$ & 99 & SUNY-STONY BROOK & $14,114,795$ \\
\hline 43 & PURDUE & $24,023,842$ & 100 & VIRGINIA TECH & $14,082,936$ \\
\hline 44 & CONNECTICUT & $23,960,782$ & 101 & OKLAHOMA STATE & $13,912,768$ \\
\hline 45 & BRIGHAM YOUNG & $23,942,505$ & 102 & OREGON & $13,739,169$ \\
\hline 46 & GEORGETOWN & $23,754,207$ & 103 & OHIO & $13,632,399$ \\
\hline 47 & MARYLAND & $23,589,122$ & 104 & MCMASTER & $13,626,100$ \\
\hline 48 & MIAMI & $23,328,442$ & 105 & WASHINGTON STATE & $13,544,201$ \\
\hline 49 & UTAH & $23,288,732$ & 106 & COLORADO STATE & $13,412,963$ \\
\hline 50 & GEORGIA & $23,014,039$ & 107 & LOUISIANA STATE & $12,864,995$ \\
\hline 51 & VANDERBILT & $23,007,106$ & 108 & AUBURN & $12,571,525$ \\
\hline 52 & CINCINNATI & $22,960,596$ & 109 & SUNY-ALBANY & $12,041,893$ \\
\hline 53 & TENNESSEE & $22,871,489$ & 110 & GUELPH & $11,413,624$ \\
\hline 54 & GEORGE WASHINGTON & $21,727,764$ & 111 & GEORGIA TECH & $11,373,366$ \\
\hline 55 & NOTRE DAME & $21,694,210$ & 112 & KENT STATE & $10,830,484$ \\
\hline 56 & MIT & $21,365,671$ & 113 & HOWARD & $9,854,124$ \\
\hline 57 & TEXAS TECH & $21,324,759$ & & & \\
\hline
\end{tabular}


RANK ORDER TABLE 10

MONOGRAPHS PURCHASED (VOLUMES)

\begin{tabular}{|c|c|c|c|c|c|}
\hline RANK & INSTITUTION & VALUe & RANK & INSTITUTION & VALUE \\
\hline 1 & YALE & 160,397 & 58 & HAWAII & 32,248 \\
\hline 2 & TORONTO & 137,067 & 59 & SOUTHERN CALIFORNIA & 32,191 \\
\hline 3 & COLUMBIA & 118,294 & 60 & VANDERBILT & 31,683 \\
\hline 4 & CHICAGO & 114,686 & 61 & COLORADO & 30,920 \\
\hline 5 & BRIGHAM YOUNG & 93,712 & 62 & CONNECTICUT & 29,929 \\
\hline 6 & OHIO STATE & 90,377 & 63 & DELAWARE & 29,101 \\
\hline 7 & TEXAS A\&M & 86,572 & 64 & OKLAHOMA & 29,096 \\
\hline 8 & CALIFORNIA, SAN DIEGO & 83,135 & 65 & DARTMOUTH & 28,985 \\
\hline 9 & PRINCETON & 81,140 & 66 & ILLINOIS, CHICAGO & 28,866 \\
\hline 10 & NEW YORK & 80,066 & 67 & IOWA STATE & 28,581 \\
\hline 11 & JOHNS HOPKINS & 72,596 & 68 & OKLAHOMA STATE & 28,095 \\
\hline 12 & PENNSYLVANIA STATE & 70,021 & 69 & CALIFORNIA, IRVINE & 28,015 \\
\hline 13 & ARIZONA STATE & 69,903 & 70 & GEORGE WASHINGTON & 27,426 \\
\hline 14 & NORTH CAROLINA & 69,290 & 71 & CALIFORNIA, SANTA BARBARA & 27,218 \\
\hline 15 & TEXAS & 68,059 & 72 & WESTERN ONTARIO & 26,961 \\
\hline 16 & DUKE & 67,985 & 73 & VIRGINIA TECH & 26,196 \\
\hline 17 & CALIFORNIA, LOS ANGELES & 65,597 & 74 & QUEEN'S & 25,950 \\
\hline 18 & ILLINOIS, URBANA & 65,445 & 75 & FLORIDA STATE & 25,699 \\
\hline 19 & INDIANA & 63,013 & 76 & TULANE & 25,590 \\
\hline 20 & IOWA & 59,854 & 77 & RUTGERS & 24,862 \\
\hline 21 & WASHINGTON & 57,155 & 78 & PURDUE & 23,907 \\
\hline 22 & LOUISVILLE & 55,477 & 79 & WASHINGTON U.-ST. LOUIS & 23,570 \\
\hline 23 & NOTRE DAME & 54,514 & 80 & NEW MEXICO & 23,221 \\
\hline 24 & MONTREAL & 52,758 & 81 & ALABAMA & 22,388 \\
\hline 25 & MARYLAND & 52,712 & 82 & MIT & 22,343 \\
\hline 26 & GEORGETOWN & 52,381 & 83 & WATERLOO & 21,567 \\
\hline 27 & TEMPLE & 51,352 & 84 & LOUISIANA STATE & 21,468 \\
\hline 28 & ARIZONA & 49,812 & 85 & SASKATCHEWAN & 21,236 \\
\hline 29 & WISCONSIN & 49,761 & 86 & SYRACUSE & 21,128 \\
\hline 30 & SOUTH CAROLINA & 47,881 & 87 & ROCHESTER & 20,591 \\
\hline 31 & GEORGIA & 47,805 & 88 & MASSACHUSETTS & 19,301 \\
\hline 32 & MANITOBA & 47,129 & 89 & AUBURN & 18,338 \\
\hline 33 & MCGILL & 46,334 & 90 & BOSTON & 18,175 \\
\hline 34 & HOUSTON & 45,522 & 91 & WAYNE STATE & 17,719 \\
\hline 35 & NORTHWESTERN & 43,803 & 92 & NEBRASKA & 17,298 \\
\hline 36 & EMORY & 42,830 & 93 & WASHINGTON STATE & 17,096 \\
\hline 37 & OREGON & 42,822 & 94 & GUELPH & 16,950 \\
\hline 38 & SOUTHERN ILLINOIS & 41,740 & 95 & SUNY-ALBANY & 16,399 \\
\hline 39 & BROWN & 40,070 & 96 & SUNY-BUFFALO & 15,316 \\
\hline 40 & CINCINNATI & 39,729 & 97 & TENNESSEE & 12,672 \\
\hline 41 & TEXAS TECH & 39,280 & 98 & CASE WESTERN RESERVE & 12,639 \\
\hline 42 & VIRGINIA & 38,954 & 99 & SUNY-STONY BROOK & 12,035 \\
\hline 43 & MINNESOTA & 38,722 & 100 & KENT STATE & 11,808 \\
\hline 44 & COLORADO STATE & 38,589 & 101 & GEORGIA TECH & 6,327 \\
\hline 45 & KANSAS & 37,802 & 102 & HOWARD & 4,670 \\
\hline 46 & RICE & 37,175 & 103 & ALBERTA & UA \\
\hline 47 & MISSOURI & 36,780 & 104 & BOSTON COLLEGE & UA \\
\hline 48 & OHIO & 36,564 & 105 & BRITISH COLUMBIA & UA \\
\hline 49 & MICHIGAN STATE & 36,418 & 106 & CALIFORNIA, BERKELEY & UA \\
\hline 50 & CALIFORNIA, RIVERSIDE & 36,001 & 107 & CALIFORNIA, DAVIS & UA \\
\hline 51 & KENTUCKY & 35,306 & 108 & CORNELL & UA \\
\hline 52 & FLORIDA & 34,584 & 109 & HARVARD & UA \\
\hline 53 & MIAMI & 33,996 & 110 & MICHIGAN & UA \\
\hline 54 & MCMASTER & 33,718 & 111 & PENNSYLVANIA & UA \\
\hline 55 & YORK & 33,158 & 112 & PITTSBURGH & UA \\
\hline 56 & NORTH CAROLINA STATE & 32,571 & 113 & UTAH & UA \\
\hline 57 & LAVAL & 32,437 & & & \\
\hline
\end{tabular}

UA - Unavailable 
RANK ORDER TABLE 11

EXPENDITURES FOR MONOGRAPHS

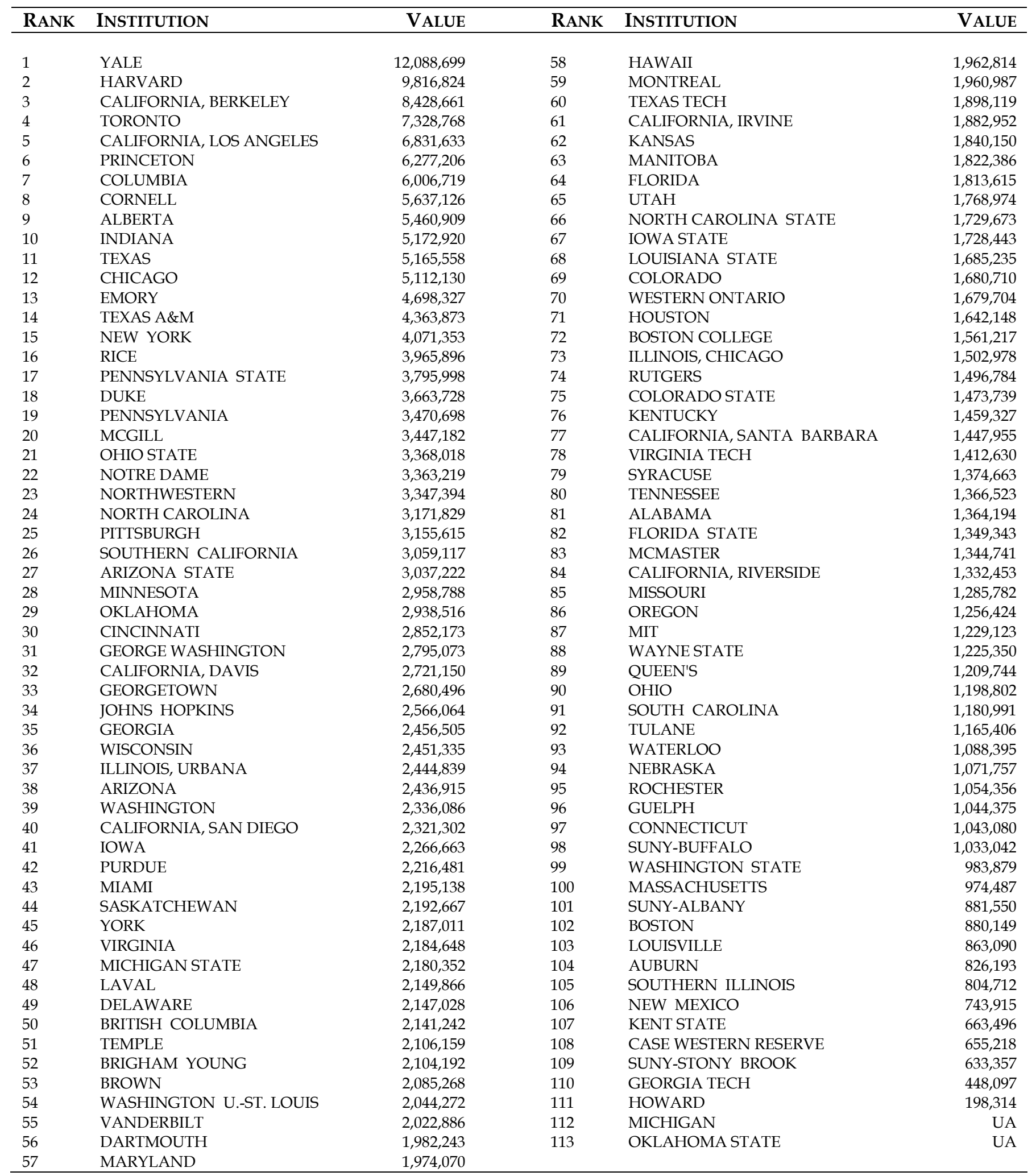

UA - Unavailable 
RANK ORDER TABLE 12

Current Serials Purchased (Subscriptions)

\begin{tabular}{|c|c|c|c|c|c|}
\hline RANK & INSTITUTION & VALUE & RANK & INSTITUTION & VALUE \\
\hline 1 & CALIFORNIA, BERKELEY & 89,249 & 58 & OHIO STATE & 21,734 \\
\hline 2 & COLUMBIA & 82,764 & 59 & TEMPLE & 21,614 \\
\hline 3 & MCGILL & 75,111 & 60 & KENTUCKY & 21,132 \\
\hline 4 & MICHIGAN & 74,746 & 61 & MANITOBA & 20,494 \\
\hline 5 & INDIANA & 71,330 & 62 & GEORGE WASHINGTON & 20,421 \\
\hline 6 & FLORIDA & 69,762 & 63 & CALIFORNIA, SANTA BARBARA & 20,237 \\
\hline 7 & YALE & 66,597 & 64 & CALIFORNIA, SAN DIEGO & 20,129 \\
\hline 8 & ILLINOIS, URBANA & 62,848 & 65 & COLORADO STATE & 20,069 \\
\hline 9 & CORNELL & 62,320 & 66 & BRIGHAM YOUNG & 19,858 \\
\hline 10 & TORONTO & 58,572 & 67 & ARIZONA STATE & 19,726 \\
\hline 11 & DUKE & 57,223 & 68 & HAWAII & 19,616 \\
\hline 12 & SOUTHERN CALIFORNIA & 56,739 & 69 & MISSOURI & 18,982 \\
\hline 13 & MONTREAL & 56,723 & 70 & HOUSTON & 18,807 \\
\hline 14 & PENNSYLVANIA STATE & 56,674 & 71 & SYRACUSE & 18,793 \\
\hline 15 & ALBERTA & 55,708 & 72 & IOWA STATE & 18,610 \\
\hline 16 & MINNESOTA & 53,126 & 73 & MCMASTER & 18,343 \\
\hline 17 & WISCONSIN & 52,339 & 74 & NEW MEXICO & 18,143 \\
\hline 18 & MIAMI & 50,290 & 75 & OREGON & 17,775 \\
\hline 19 & FLORIDA STATE & 50,074 & 76 & SUNY-ALBANY & 17,524 \\
\hline 20 & RUTGERS & 50,003 & 77 & $\mathrm{OHIO}$ & 16,814 \\
\hline 21 & DARTMOUTH & 49,274 & 78 & SOUTH CAROLINA & 15,713 \\
\hline 22 & JOHNS HOPKINS & 48,631 & 79 & PURDUE & 14,154 \\
\hline 23 & NEBRASKA & 44,936 & 80 & GEORGIA TECH & 12,682 \\
\hline 24 & WESTERN ONTARIO & 44,122 & 81 & MARYLAND & 12,147 \\
\hline 25 & NEW YORK & 44,005 & 82 & KENT STATE & 11,941 \\
\hline 26 & CONNECTICUT & 41,626 & 83 & MIT & 11,826 \\
\hline 27 & CINCINNATI & 40,890 & 84 & COLORADO & 11,623 \\
\hline 28 & PRINCETON & 40,498 & 85 & GUELPH & 11,304 \\
\hline 29 & BOSTON COLLEGE & 40,204 & 86 & CASE WESTERN RESERVE & 11,228 \\
\hline 30 & QUEEN'S & 39,918 & 87 & RICE & 11,157 \\
\hline 31 & LOUISVILLE & 37,931 & 88 & AUBURN & 10,072 \\
\hline 32 & OKLAHOMA & 37,840 & 89 & HOWARD & 9,823 \\
\hline 33 & TEXAS TECH & 37,711 & 90 & DELAWARE & 7,519 \\
\hline 34 & CALIFORNIA, IRVINE & 35,648 & 91 & ARIZONA & UA \\
\hline 35 & TEXAS A\&M & 34,567 & 92 & BOSTON & UA \\
\hline 36 & MASSACHUSETTS & 33,959 & 93 & BRITISH COLUMBIA & UA \\
\hline 37 & VIRGINIA TECH & 33,874 & 94 & CALIFORNIA, DAVIS & UA \\
\hline 38 & IOWA & 32,909 & 95 & CALIFORNIA, LOS ANGELES & UA \\
\hline 39 & NORTH CAROLINA & 30,796 & 96 & CHICAGO & UA \\
\hline 40 & WASHINGTON U.-ST. LOUIS & 28,490 & 97 & HARVARD & UA \\
\hline 41 & SUNY-STONY BROOK & 27,895 & 98 & ILLINOIS, CHICAGO & UA \\
\hline 42 & VANDERBILT & 26,999 & 99 & LOUISIANA STATE & UA \\
\hline 43 & GEORGIA & 26,482 & 100 & NORTHWESTERN & UA \\
\hline 44 & BROWN & 26,373 & 101 & NOTRE DAME & UA \\
\hline 45 & SASKATCHEWAN & 25,996 & 102 & OKLAHOMA STATE & UA \\
\hline 46 & GEORGETOWN & 25,937 & 103 & PENNSYLVANIA & UA \\
\hline 47 & ALABAMA & 25,904 & 104 & PITTSBURGH & UA \\
\hline 48 & KANSAS & 25,663 & 105 & ROCHESTER & UA \\
\hline 49 & TENNESSEE & 25,567 & 106 & TEXAS & UA \\
\hline 50 & VIRGINIA & 24,386 & 107 & TULANE & UA \\
\hline 51 & EMORY & 24,316 & 108 & UTAH & UA \\
\hline 52 & LAVAL & 23,897 & 109 & WASHINGTON & UA \\
\hline 53 & SUNY-BUFFALO & 23,800 & 110 & WASHINGTON STATE & UA \\
\hline 54 & NORTH CAROLINA STATE & 22,794 & 111 & WATERLOO & UA \\
\hline 55 & MICHIGAN STATE & 22,767 & 112 & WAYNE STATE & UA \\
\hline 56 & SOUTHERN ILLINOIS & 22,204 & 113 & YORK & UA \\
\hline 57 & CALIFORNIA, RIVERSIDE & 21,874 & & & \\
\hline
\end{tabular}

UA - Unavailable 


\section{RANK ORDER TABLE 13}

\section{EXPENDITURES FOR CURRENT SERIALS}

\begin{tabular}{|c|c|c|c|c|c|}
\hline RANK & INSTITUTION & VALUE & RANK & INSTITUTION & VALUE \\
\hline 1 & COLUMBIA & $12,831,465$ & 58 & BOSTON COLLEGE & $6,143,117$ \\
\hline 2 & PENNSYLVANIA STATE & $12,249,954$ & 59 & OKLAHOMA & $6,136,135$ \\
\hline 3 & CHICAGO & $11,056,788$ & 60 & QUEEN'S & $6,114,253$ \\
\hline 4 & NEW YORK & $10,900,902$ & 61 & WAYNE STATE & $6,079,545$ \\
\hline 5 & TORONTO & $10,010,684$ & 62 & YORK & $6,057,219$ \\
\hline 6 & PENNSYLVANIA & $10,005,808$ & 63 & EMORY & $5,903,787$ \\
\hline 7 & HARVARD & $9,911,522$ & 64 & NORTH CAROLINA STATE & $5,813,216$ \\
\hline 8 & BRITISH COLUMBIA & $9,899,713$ & 65 & SOUTH CAROLINA & $5,717,091$ \\
\hline 9 & MINNESOTA & $9,859,444$ & 66 & SOUTHERN ILLINOIS & $5,698,153$ \\
\hline 10 & WASHINGTON & $9,818,882$ & 67 & HOUSTON & $5,684,365$ \\
\hline 11 & JOHNS HOPKINS & $9,491,228$ & 68 & DARTMOUTH & $5,669,207$ \\
\hline 12 & TEXAS A\&M & $9,385,580$ & 69 & HAWAII & $5,648,264$ \\
\hline 13 & TENNESSEE & $9,261,449$ & 70 & GEORGE WASHINGTON & $5,633,307$ \\
\hline 14 & DUKE & $9,201,728$ & 71 & VIRGINIA TECH & $5,546,834$ \\
\hline 15 & MIAMI & $8,946,033$ & 72 & UTAH & $5,541,181$ \\
\hline 16 & CALIFORNIA, BERKELEY & $8,719,614$ & 73 & KANSAS & $5,483,771$ \\
\hline 17 & ILLINOIS, URBANA & $8,634,385$ & 74 & BOSTON & $5,443,573$ \\
\hline 18 & TEXAS & $8,610,285$ & 75 & SASKATCHEWAN & $5,407,965$ \\
\hline 19 & ALBERTA & $8,492,244$ & 76 & CALIFORNIA, IRVINE & $5,392,865$ \\
\hline 20 & FLORIDA & $8,347,943$ & 77 & NEBRASKA & $5,386,159$ \\
\hline 21 & VANDERBILT & $8,260,264$ & 78 & CALIFORNIA, SAN DIEGO & $5,380,965$ \\
\hline 22 & NORTH CAROLINA & $8,151,067$ & 79 & BROWN & $5,343,805$ \\
\hline 23 & MONTREAL & $8,124,191$ & 80 & ALABAMA & $5,339,198$ \\
\hline 24 & LOUISVILLE & $8,076,155$ & 81 & RICE & $5,295,592$ \\
\hline 25 & ARIZONA & $8,015,246$ & 82 & DELAWARE & $5,283,118$ \\
\hline 26 & PITTSBURGH & $7,881,684$ & 83 & ROCHESTER & $5,269,503$ \\
\hline 27 & NORTHWESTERN & $7,878,978$ & 84 & CASE WESTERN RESERVE & $5,226,735$ \\
\hline 28 & CALIFORNIA, LOS ANGELES & $7,790,034$ & 85 & NOTRE DAME & $5,167,892$ \\
\hline 29 & YALE & $7,705,342$ & 86 & SUNY-STONY BROOK & $5,068,744$ \\
\hline 30 & GEORGIA & $7,620,806$ & 87 & TULANE & $5,039,744$ \\
\hline 31 & RUTGERS & $7,499,482$ & 88 & TEMPLE & $5,037,468$ \\
\hline 32 & WESTERN ONTARIO & $7,490,733$ & 89 & MISSOURI & $4,989,546$ \\
\hline 33 & PRINCETON & $7,480,441$ & 90 & MCMASTER & $4,840,846$ \\
\hline 34 & ARIZONA STATE & $7,437,248$ & 91 & FLORIDA STATE & $4,804,159$ \\
\hline 35 & IOWA & $7,343,041$ & 92 & AUBURN & $4,647,678$ \\
\hline 36 & CORNELL & $7,340,695$ & 93 & LOUISIANA STATE & $4,608,991$ \\
\hline 37 & INDIANA & $7,326,574$ & 94 & MARYLAND & $4,599,063$ \\
\hline 38 & MICHIGAN STATE & $7,295,789$ & 95 & CALIFORNIA, DAVIS & $4,569,504$ \\
\hline 39 & WASHINGTON U.-ST. LOUIS & $7,275,964$ & 96 & WATERLOO & $4,540,404$ \\
\hline 40 & KENTUCKY & $7,265,268$ & 97 & MANITOBA & $4,528,145$ \\
\hline 41 & SOUTHERN CALIFORNIA & $7,215,984$ & 98 & CALIFORNIA, RIVERSIDE & $4,467,954$ \\
\hline 42 & BRIGHAM YOUNG & $7,000,979$ & 99 & WASHINGTON STATE & $4,453,232$ \\
\hline 43 & OHIO STATE & $6,953,106$ & 100 & SYRACUSE & $4,444,275$ \\
\hline 44 & GEORGETOWN & $6,863,022$ & 101 & MASSACHUSETTS & $4,339,742$ \\
\hline 45 & TEXAS TECH & $6,849,349$ & 102 & COLORADO STATE & $4,131,559$ \\
\hline 46 & VIRGINIA & $6,741,512$ & 103 & GEORGIA TECH & $4,105,463$ \\
\hline 47 & MCGILL & $6,699,174$ & 104 & NEW MEXICO & $4,055,092$ \\
\hline 48 & WISCONSIN & $6,629,327$ & 105 & SUNY-ALBANY & $4,030,434$ \\
\hline 49 & PURDUE & $6,600,726$ & 106 & CALIFORNIA, SANTA BARBARA & $3,788,375$ \\
\hline 50 & CINCINNATI & $6,562,896$ & 107 & OHIO & $3,651,914$ \\
\hline 51 & IOWA STATE & $6,539,217$ & 108 & GUELPH & $3,245,327$ \\
\hline 52 & LAVAL & $6,389,065$ & 109 & OREGON & $3,070,990$ \\
\hline 53 & CONNECTICUT & $6,369,593$ & 110 & KENT STATE & $2,940,211$ \\
\hline 54 & ILLINOIS, CHICAGO & $6,353,550$ & 111 & HOWARD & $2,923,720$ \\
\hline 55 & MIT & $6,324,816$ & 112 & MICHIGAN & UA \\
\hline 56 & COLORADO & $6,289,768$ & 113 & OKLAHOMA STATE & UA \\
\hline 57 & SUNY-BUFFALO & $6,270,231$ & & & \\
\hline
\end{tabular}

UA - Unavailable 


\section{RANK ORDER TABLE 14 TOTAL ITEMS LOANED (ILL/DD)}

\begin{tabular}{|c|c|c|c|c|c|}
\hline RANK & INSTITUTION & VALUE & RANK & INSTITUTION & VALUE \\
\hline 1 & MINNESOTA & 177,020 & 58 & GEORGE WASHINGTON & 34,591 \\
\hline 2 & WISCONSIN & 115,302 & 59 & PRINCETON & 34,402 \\
\hline 3 & OHIO STATE & 108,828 & 60 & DARTMOUTH & 34,249 \\
\hline 4 & INDIANA & 96,126 & 61 & RUTGERS & 34,055 \\
\hline 5 & CINCINNATI & 93,770 & 62 & IOWA STATE & 33,827 \\
\hline 6 & ILLINOIS, URBANA & 83,999 & 63 & TEXAS TECH & 33,632 \\
\hline 7 & CASE WESTERN RESERVE & 81,645 & 64 & CALIFORNIA, SAN DIEGO & 33,040 \\
\hline 8 & ALBERTA & 80,302 & 65 & PURDUE & 31,666 \\
\hline 9 & ILLINOIS, CHICAGO & 76,941 & 66 & KENTUCKY & 31,662 \\
\hline 10 & $\mathrm{OHIO}$ & 76,678 & 67 & HOUSTON & 31,019 \\
\hline 11 & WASHINGTON & 72,447 & 68 & FLORIDA & 28,979 \\
\hline 12 & OREGON & 69,623 & 69 & VANDERBILT & 28,686 \\
\hline 13 & WATERLOO & 68,736 & 70 & CALIFORNIA, IRVINE & 28,501 \\
\hline 14 & PITTSBURGH & 68,125 & 71 & CALIFORNIA, DAVIS & 28,305 \\
\hline 15 & PENNSYLVANIA & 64,483 & 72 & NEBRASKA & 27,857 \\
\hline 16 & IOWA & 63,356 & 73 & MONTREAL & 27,664 \\
\hline 17 & MISSOURI & 62,761 & 74 & BRITISH COLUMBIA & 26,402 \\
\hline 18 & PENNSYLVANIA STATE & 61,885 & 75 & ROCHESTER & 25,694 \\
\hline 19 & CORNELL & 61,490 & 76 & NEW YORK & 25,215 \\
\hline 20 & ARIZONA & 61,151 & 77 & SOUTHERN CALIFORNIA & 24,385 \\
\hline 21 & COLORADO STATE & 60,998 & 78 & SUNY-STONY BROOK & 24,294 \\
\hline 22 & DUKE & 59,964 & 79 & NOTRE DAME & 23,822 \\
\hline 23 & MASSACHUSETTS & 59,838 & 80 & SASKATCHEWAN & 23,758 \\
\hline 24 & EMORY & 59,096 & 81 & NEW MEXICO & 23,540 \\
\hline 25 & KANSAS & 58,466 & 82 & CALIFORNIA, SANTA BARBARA & 23,432 \\
\hline 26 & SOUTHERN ILLINOIS & 57,968 & 83 & QUEEN'S & 23,016 \\
\hline 27 & NORTH CAROLINA & 56,504 & 84 & TEMPLE & 22,832 \\
\hline 28 & ARIZONA STATE & 56,361 & 85 & CALIFORNIA, RIVERSIDE & 22,618 \\
\hline 29 & CALIFORNIA, LOS ANGELES & 55,454 & 86 & LOUISVILLE & 22,570 \\
\hline 30 & CHICAGO & 50,646 & 87 & FLORIDA STATE & 22,392 \\
\hline 31 & COLUMBIA & 50,096 & 88 & MANITOBA & 22,026 \\
\hline 32 & YALE & 49,320 & 89 & VIRGINIA TECH & 21,978 \\
\hline 33 & GEORGIA & 49,233 & 90 & SOUTH CAROLINA & 21,813 \\
\hline 34 & WASHINGTON U.-ST. LOUIS & 48,775 & 91 & LAVAL & 20,088 \\
\hline 35 & COLORADO & 48,208 & 92 & WESTERN ONTARIO & 19,825 \\
\hline 36 & TEXAS & 48,065 & 93 & BOSTON COLLEGE & 18,867 \\
\hline 37 & MICHIGAN STATE & 47,915 & 94 & MCGILL & 17,375 \\
\hline 38 & GUELPH & 47,732 & 95 & MARYLAND & 16,747 \\
\hline 39 & CONNECTICUT & 46,537 & 96 & DELAWARE & 16,491 \\
\hline 40 & WASHINGTON STATE & 46,040 & 97 & BOSTON & 16,230 \\
\hline 41 & HARVARD & 45,097 & 98 & MCMASTER & 16,061 \\
\hline 42 & TEXAS A\&M & 45,016 & 99 & YORK & 14,832 \\
\hline 43 & SUNY-BUFFALO & 44,521 & 100 & SYRACUSE & 14,230 \\
\hline 44 & VIRGINIA & 44,397 & 101 & SUNY-ALBANY & 13,921 \\
\hline 45 & NORTHWESTERN & 43,569 & 102 & RICE & 13,802 \\
\hline 46 & CALIFORNIA, BERKELEY & 42,662 & 103 & KENT STATE & 13,710 \\
\hline 47 & MIAMI & 41,279 & 104 & NORTH CAROLINA STATE & 13,622 \\
\hline 48 & UTAH & 39,396 & 105 & AUBURN & 13,113 \\
\hline 49 & OKLAHOMA STATE & 39,036 & 106 & MIT & 11,261 \\
\hline 50 & WAYNE STATE & 38,802 & 107 & ALABAMA & 10,482 \\
\hline 51 & GEORGETOWN & 38,388 & 108 & GEORGIA TECH & 9,852 \\
\hline 52 & BROWN & 36,904 & 109 & LOUISIANA STATE & 7,733 \\
\hline 53 & BRIGHAM YOUNG & 36,103 & 110 & HOWARD & 5,676 \\
\hline 54 & JOHNS HOPKINS & 36,097 & 111 & TULANE & 4,411 \\
\hline 55 & TENNESSEE & 36,035 & 112 & HAWAII & 4,394 \\
\hline 56 & OKLAHOMA & 35,697 & 113 & MICHIGAN & UA \\
\hline 57 & TORONTO & 35,439 & & & \\
\hline
\end{tabular}

UA - Unavailable 


\section{RANK ORDER TABLE 15 \\ TOTAL ITEMS BORROWED (ILL/DD)}

\begin{tabular}{|c|c|c|c|c|c|}
\hline RANK & INSTITUTION & VALUe & RANK & INSTITUTION & VALUE \\
\hline 1 & OHIO STATE & 93,669 & 58 & UTAH & 26,609 \\
\hline 2 & ILLINOIS, URBANA & 87,264 & 59 & NORTH CAROLINA STATE & 25,006 \\
\hline 3 & PENNSYLVANIA & 79,534 & 60 & KENTUCKY & 24,392 \\
\hline 4 & WISCONSIN & 75,331 & 61 & BRITISH COLUMBIA & 24,371 \\
\hline 5 & OREGON & 63,238 & 62 & DELAWARE & 24,330 \\
\hline 6 & GUELPH & 62,831 & 63 & GEORGETOWN & 24,320 \\
\hline 7 & COLORADO STATE & 61,965 & 64 & PITTSBURGH & 23,914 \\
\hline 8 & TEXAS A\&M & 58,344 & 65 & LOUISIANA STATE & 23,341 \\
\hline 9 & GEORGE WASHINGTON & 56,506 & 66 & OKLAHOMA STATE & 23,077 \\
\hline 10 & WASHINGTON & 55,011 & 67 & NEW MEXICO & 22,995 \\
\hline 11 & OHIO & 54,503 & 68 & CHICAGO & 22,652 \\
\hline 12 & INDIANA & 51,920 & 69 & CALIFORNIA, BERKELEY & 22,374 \\
\hline 13 & PENNSYLVANIA STATE & 51,789 & 70 & VIRGINIA TECH & 22,079 \\
\hline 14 & CINCINNATI & 51,142 & 71 & ROCHESTER & 21,834 \\
\hline 15 & COLUMBIA & 50,297 & 72 & SYRACUSE & 21,179 \\
\hline 16 & WASHINGTON U.-ST. LOUIS & 48,880 & 73 & SASKATCHEWAN & 21,119 \\
\hline 17 & WATERLOO & 46,413 & 74 & MCGILL & 20,656 \\
\hline 18 & KANSAS & 42,771 & 75 & NEBRASKA & 20,172 \\
\hline 19 & CASE WESTERN RESERVE & 42,151 & 76 & MONTREAL & 20,116 \\
\hline 20 & MARYLAND & 40,366 & 77 & FLORIDA & 20,001 \\
\hline 21 & MISSOURI & 39,219 & 78 & VANDERBILT & 19,610 \\
\hline 22 & BRIGHAM YOUNG & 38,989 & 79 & HOUSTON & 19,606 \\
\hline 23 & YALE & 38,237 & 80 & TEMPLE & 19,391 \\
\hline 24 & BROWN & 37,673 & 81 & SOUTH CAROLINA & 18,794 \\
\hline 25 & ALBERTA & 37,333 & 82 & WASHINGTON STATE & 18,426 \\
\hline 26 & ILLINOIS, CHICAGO & 36,930 & 83 & SUNY-ALBANY & 18,285 \\
\hline 27 & SOUTHERN ILLINOIS & 36,342 & 84 & EMORY & 17,843 \\
\hline 28 & MINNESOTA & 36,326 & 85 & HAWAII & 17,676 \\
\hline 29 & JOHNS HOPKINS & 36,266 & 86 & NOTRE DAME & 17,667 \\
\hline 30 & MASSACHUSETTS & 36,190 & 87 & CALIFORNIA, RIVERSIDE & 17,511 \\
\hline 31 & MICHIGAN STATE & 35,221 & 88 & SUNY-BUFFALO & 17,064 \\
\hline 32 & CORNELL & 34,587 & 89 & FLORIDA STATE & 16,263 \\
\hline 33 & CALIFORNIA, LOS ANGELES & 34,529 & 90 & MIAMI & 16,110 \\
\hline 34 & TEXAS & 34,428 & 91 & SOUTHERN CALIFORNIA & 16,103 \\
\hline 35 & OKLAHOMA & 33,878 & 92 & BOSTON COLLEGE & 16,094 \\
\hline 36 & PRINCETON & 33,579 & 93 & SUNY-STONY BROOK & 15,576 \\
\hline 37 & CALIFORNIA, SANTA BARBARA & 32,628 & 94 & NORTH CAROLINA & 14,934 \\
\hline 38 & VIRGINIA & 32,501 & 95 & MCMASTER & 14,840 \\
\hline 39 & LOUISVILLE & 32,090 & 96 & BOSTON & 14,546 \\
\hline 40 & COLORADO & 31,708 & 97 & IOWA STATE & 14,450 \\
\hline 41 & MANITOBA & 31,600 & 98 & HARVARD & 13,381 \\
\hline 42 & WAYNE STATE & 30,880 & 99 & QUEEN'S & 13,118 \\
\hline 43 & DUKE & 30,613 & 100 & LAVAL & 12,940 \\
\hline 44 & NORTHWESTERN & 30,510 & 101 & MIT & 12,558 \\
\hline 45 & DARTMOUTH & 30,309 & 102 & ALABAMA & 10,986 \\
\hline 46 & CALIFORNIA, DAVIS & 29,978 & 103 & RICE & 10,758 \\
\hline 47 & ARIZONA STATE & 29,649 & 104 & TORONTO & 10,748 \\
\hline 48 & TENNESSEE & 29,382 & 105 & AUBURN & 9,391 \\
\hline 49 & CONNECTICUT & 29,347 & 106 & GEORGIA & 8,698 \\
\hline 50 & CALIFORNIA, IRVINE & 29,287 & 107 & GEORGIA TECH & 8,634 \\
\hline 51 & CALIFORNIA, SAN DIEGO & 29,220 & 108 & KENT STATE & 7,725 \\
\hline 52 & NEW YORK & 29,160 & 109 & HOWARD & 7,239 \\
\hline 53 & IOWA & 28,761 & 110 & TULANE & 6,755 \\
\hline 54 & PURDUE & 28,274 & 111 & WESTERN ONTARIO & 6,721 \\
\hline 55 & ARIZONA & 27,725 & 112 & YORK & 4,926 \\
\hline 56 & RUTGERS & 27,412 & 113 & MICHIGAN & UA \\
\hline 57 & TEXAS TECH & 26,959 & & & \\
\hline
\end{tabular}

UA - Unavaileble 
Rank ORder Table 16

Professional STAFF (FTE)

\begin{tabular}{|c|c|c|c|c|c|}
\hline RANK & INSTITUTION & VALUE & RANK & INSTITUTION & VALUE \\
\hline 1 & HARVARD & 554 & 58 & MICHIGAN STATE & 79 \\
\hline 2 & COLUMBIA & 311 & 59 & UTAH & 76 \\
\hline 3 & YALE & 260 & 60 & GEORGE WASHINGTON & 75 \\
\hline 4 & WISCONSIN & 233 & 61 & HAWAII & 75 \\
\hline 5 & CALIFORNIA, BERKELEY & 219 & 62 & BOSTON COLLEGE & 73 \\
\hline 6 & WASHINGTON & 189 & 63 & TEMPLE & 73 \\
\hline 7 & TORONTO & 187 & 64 & NEW MEXICO & 72 \\
\hline 8 & ILLINOIS, URBANA & 185 & 65 & BROWN & 71 \\
\hline 9 & CALIFORNIA, LOS ANGELES & 174 & 66 & GEORGIA & 71 \\
\hline 10 & DUKE & 173 & 67 & WESTERN ONTARIO & 71 \\
\hline 11 & MICHIGAN & 158 & 68 & FLORIDA STATE & 70 \\
\hline 12 & INDIANA & 154 & 69 & SOUTH CAROLINA & 68 \\
\hline 13 & PENNSYLVANIA STATE & 148 & 70 & NOTRE DAME & 67 \\
\hline 14 & NORTH CAROLINA & 147 & 71 & SYRACUSE & 67 \\
\hline 15 & MARYLAND & 145 & 72 & CALIFORNIA, IRVINE & 66 \\
\hline 16 & PRINCETON & 143 & 73 & CHICAGO & 66 \\
\hline 17 & TEXAS & 143 & 74 & PURDUE & 66 \\
\hline 18 & JOHNS HOPKINS & 140 & 75 & YORK & 66 \\
\hline 19 & SUNY-BUFFALO & 134 & 76 & CALIFORNIA, DAVIS & 65 \\
\hline 20 & TEXAS A\&M & 131 & 77 & $\mathrm{OHIO}$ & 64 \\
\hline 21 & NEW YORK & 125 & 78 & ALABAMA & 63 \\
\hline 22 & OHIO STATE & 125 & 79 & CASE WESTERN RESERVE & 62 \\
\hline 23 & BRIGHAM YOUNG & 120 & 80 & HOUSTON & 62 \\
\hline 24 & PENNSYLVANIA & 120 & 81 & MCGILL & 62 \\
\hline 25 & PITTSBURGH & 120 & 82 & OKLAHOMA STATE & 62 \\
\hline 26 & CORNELL & 118 & 83 & DARTMOUTH & 61 \\
\hline 27 & NORTH CAROLINA STATE & 117 & 84 & MANITOBA & 61 \\
\hline 28 & NORTHWESTERN & 115 & 85 & DELAWARE & 60 \\
\hline 29 & SOUTHERN CALIFORNIA & 113 & 86 & LAVAL & 60 \\
\hline 30 & WASHINGTON U.-ST. LOUIS & 113 & 87 & MISSOURI & 60 \\
\hline 31 & BRITISH COLUMBIA & 112 & 88 & OKLAHOMA & 60 \\
\hline 32 & FLORIDA & 109 & 89 & SUNY-ALBANY & 60 \\
\hline 33 & MONTREAL & 108 & 90 & RICE & 59 \\
\hline 34 & MINNESOTA & 106 & 91 & OREGON & 57 \\
\hline 35 & CINCINNATI & 105 & 92 & MASSACHUSETTS & 55 \\
\hline 36 & EMORY & 103 & 93 & KENT STATE & 54 \\
\hline 37 & KANSAS & 103 & 94 & LOUISIANA STATE & 54 \\
\hline 38 & RUTGERS & 103 & 95 & COLORADO & 52 \\
\hline 39 & TEXAS TECH & 101 & 96 & HOWARD & 52 \\
\hline 40 & VANDERBILT & 101 & 97 & NEBRASKA & 52 \\
\hline 41 & ARIZONA & 98 & 98 & SOUTHERN ILLINOIS & 52 \\
\hline 42 & BOSTON & 98 & 99 & AUBURN & 51 \\
\hline 43 & MIT & 97 & 100 & IOWA STATE & 51 \\
\hline 44 & VIRGINIA & 97 & 101 & LOUISVILLE & 51 \\
\hline 45 & WAYNE STATE & 96 & 102 & TULANE & 51 \\
\hline 46 & SUNY-STONY BROOK & 95 & 103 & COLORADO STATE & 49 \\
\hline 47 & TENNESSEE & 95 & 104 & WASHINGTON STATE & 49 \\
\hline 48 & KENTUCKY & 91 & 105 & CALIFORNIA, SANTA BARBARA & 48 \\
\hline 49 & ROCHESTER & 91 & 106 & GEORGIA TECH & 48 \\
\hline 50 & IOWA & 90 & 107 & SASKATCHEWAN & 47 \\
\hline 51 & GEORGETOWN & 89 & 108 & QUEEN'S & 45 \\
\hline 52 & ARIZONA STATE & 88 & 109 & CALIFORNIA, RIVERSIDE & 43 \\
\hline 53 & CONNECTICUT & 88 & 110 & GUELPH & 40 \\
\hline 54 & CALIFORNIA, SAN DIEGO & 87 & 111 & VIRGINIA TECH & 36 \\
\hline 55 & ALBERTA & 82 & 112 & WATERLOO & 35 \\
\hline 56 & MIAMI & 82 & 113 & MCMASTER & 34 \\
\hline 57 & ILLINOIS, CHICAGO & 80 & & & \\
\hline
\end{tabular}


RANK ORDER TABLE 17

SUPPORT STAFF (FTE)

\begin{tabular}{|c|c|c|c|c|c|}
\hline RANK & INSTITUTION & VALUE & RANK & INSTITUTION & VAlue \\
\hline 1 & HARVARD & 580 & 58 & MICHIGAN STATE & 124 \\
\hline 2 & PENNSYLVANIA STATE & 388 & 59 & KENTUCKY & 119 \\
\hline 3 & YALE & 375 & 60 & TEXAS TECH & 117 \\
\hline 4 & TORONTO & 352 & 61 & DARTMOUTH & 117 \\
\hline 5 & CORNELL & 320 & 62 & MISSOURI & 117 \\
\hline 6 & MICHIGAN & 310 & 63 & ILLINOIS, CHICAGO & 115 \\
\hline 7 & CALIFORNIA, LOS ANGELES & 297 & 64 & NEBRASKA & 114 \\
\hline 8 & TEXAS & 286 & 65 & COLORADO & 111 \\
\hline 9 & MONTREAL & 274 & 66 & KANSAS & 110 \\
\hline 10 & COLUMBIA & 247 & 67 & DELAWARE & 110 \\
\hline 11 & RUTGERS & 235 & 68 & QUEEN'S & 108 \\
\hline 12 & NEW YORK & 232 & 69 & MCMASTER & 107 \\
\hline 13 & VIRGINIA & 217 & 70 & NORTH CAROLINA STATE & 106 \\
\hline 14 & ALBERTA & 215 & 71 & VANDERBILT & 106 \\
\hline 15 & MINNESOTA & 214 & 72 & BROWN & 105 \\
\hline 16 & WASHINGTON & 212 & 73 & WESTERN ONTARIO & 103 \\
\hline 17 & PRINCETON & 210 & 74 & SOUTH CAROLINA & 101 \\
\hline 18 & NORTH CAROLINA & 204 & 75 & BOSTON & 100 \\
\hline 19 & CALIFORNIA, BERKELEY & 202 & 76 & OKLAHOMA & 99 \\
\hline 20 & FLORIDA & 202 & 77 & WATERLOO & 99 \\
\hline 21 & BRITISH COLUMBIA & 199 & 78 & MARYLAND & 97 \\
\hline 22 & ILLINOIS, URBANA & 197 & 79 & TEMPLE & 96 \\
\hline 23 & ARIZONA STATE & 194 & 80 & LOUISIANA STATE & 96 \\
\hline 24 & CHICAGO & 193 & 81 & CALIFORNIA, RIVERSIDE & 96 \\
\hline 25 & UTAH & 190 & 82 & MIT & 95 \\
\hline 26 & INDIANA & 185 & 83 & OREGON & 94 \\
\hline 27 & GEORGIA & 185 & 84 & SASKATCHEWAN & 94 \\
\hline 28 & CALIFORNIA, SAN DIEGO & 183 & 85 & HAWAII & 93 \\
\hline 29 & OHIO STATE & 179 & 86 & HOUSTON & 93 \\
\hline 30 & WISCONSIN & 172 & 87 & SOUTHERN ILLINOIS & 93 \\
\hline 31 & PITTSBURGH & 172 & 88 & IOWA STATE & 93 \\
\hline 32 & PENNSYLVANIA & 170 & 89 & WAYNE STATE & 90 \\
\hline 33 & NEW MEXICO & 166 & 90 & LOUISVILLE & 90 \\
\hline 34 & MCGILL & 163 & 91 & VIRGINIA TECH & 90 \\
\hline 35 & NOTRE DAME & 158 & 92 & WASHINGTON STATE & 84 \\
\hline 36 & JOHNS HOPKINS & 157 & 93 & ALABAMA & 82 \\
\hline 37 & DUKE & 156 & 94 & TULANE & 82 \\
\hline 38 & CALIFORNIA, DAVIS & 154 & 95 & OKLAHOMA STATE & 80 \\
\hline 39 & LAVAL & 154 & 96 & BOSTON COLLEGE & 77 \\
\hline 40 & ARIZONA & 153 & 97 & MASSACHUSETTS & 76 \\
\hline 41 & EMORY & 148 & 98 & CONNECTICUT & 75 \\
\hline 42 & NORTHWESTERN & 140 & 99 & GUELPH & 72 \\
\hline 43 & TENNESSEE & 140 & 100 & HOWARD & 71 \\
\hline 44 & CALIFORNIA, SANTA BARBARA & 138 & 101 & GEORGIA TECH & 71 \\
\hline 45 & TEXAS A\&M & 137 & 102 & CINCINNATI & 70 \\
\hline 46 & IOWA & 137 & 103 & $\mathrm{OHIO}$ & 68 \\
\hline 47 & WASHINGTON U.-ST. LOUIS & 135 & 104 & SUNY-ALBANY & 67 \\
\hline 48 & SYRACUSE & 134 & 105 & ROCHESTER & 66 \\
\hline 49 & SOUTHERN CALIFORNIA & 131 & 106 & BRIGHAM YOUNG & 61 \\
\hline 50 & GEORGE WASHINGTON & 130 & 107 & RICE & 61 \\
\hline 51 & GEORGETOWN & 129 & 108 & AUBURN & 55 \\
\hline 52 & CALIFORNIA, IRVINE & 128 & 109 & CASE WESTERN RESERVE & 53 \\
\hline 53 & YORK & 127 & 110 & COLORADO STATE & 51 \\
\hline 54 & MANITOBA & 127 & 111 & SUNY-BUFFALO & 47 \\
\hline 55 & FLORIDA STATE & 126 & 112 & KENT STATE & 38 \\
\hline 56 & PURDUE & 126 & 113 & SUNY-STONY BROOK & 25 \\
\hline 57 & MIAMI & 125 & & & \\
\hline
\end{tabular}




\section{RANK ORDER TABLE 18}

TOTAL STAFF (FTE)

\begin{tabular}{|c|c|c|c|c|c|}
\hline RANK & INSTITUTION & VALUE & RANK & INSTITUTION & VALUE \\
\hline 1 & HARVARD & 1,266 & 58 & KENTUCKY & 257 \\
\hline 2 & YALE & 706 & 59 & MIAMI & 257 \\
\hline 3 & COLUMBIA & 698 & 60 & MCGILL & 256 \\
\hline 4 & TORONTO & 688 & 61 & YORK & 252 \\
\hline 5 & CALIFORNIA, LOS ANGELES & 622 & 62 & PURDUE & 251 \\
\hline 6 & PENNSYLVANIA STATE & 612 & 63 & SUNY-BUFFALO & 249 \\
\hline 7 & CALIFORNIA, BERKELEY & 580 & 64 & FLORIDA STATE & 246 \\
\hline 8 & WISCONSIN & 577 & 65 & VANDERBILT & 245 \\
\hline 9 & MICHIGAN & 574 & 66 & CALIFORNIA, IRVINE & 243 \\
\hline 10 & CORNELL & 553 & 67 & CALIFORNIA, SANTA BARBARA & 234 \\
\hline 11 & TEXAS & 536 & 68 & TEMPLE & 229 \\
\hline 12 & WASHINGTON & 527 & 69 & CINCINNATI & 228 \\
\hline 13 & ILLINOIS, URBANA & 503 & 70 & OKLAHOMA & 228 \\
\hline 14 & INDIANA & 486 & 71 & SYRACUSE & 228 \\
\hline 15 & MARYLAND & 455 & 72 & ILLINOIS, CHICAGO & 226 \\
\hline 16 & NEW YORK & 451 & 73 & LAVAL & 226 \\
\hline 17 & FLORIDA & 439 & 74 & HAWAII & 220 \\
\hline 18 & RUTGERS & 438 & 75 & CONNECTICUT & 218 \\
\hline 19 & NORTH CAROLINA & 437 & 76 & MANITOBA & 218 \\
\hline 20 & OHIO STATE & 435 & 77 & ROCHESTER & 214 \\
\hline 21 & MINNESOTA & 408 & 78 & COLORADO & 213 \\
\hline 22 & PRINCETON & 397 & 79 & OKLAHOMA STATE & 212 \\
\hline 23 & PENNSYLVANIA & 393 & 80 & MISSOURI & 211 \\
\hline 24 & MONTREAL & 389 & 81 & MIT & 209 \\
\hline 25 & BRIGHAM YOUNG & 380 & 82 & NEBRASKA & 209 \\
\hline 26 & SOUTHERN CALIFORNIA & 375 & 83 & BROWN & 208 \\
\hline 27 & VIRGINIA & 373 & 84 & OREGON & 208 \\
\hline 28 & TEXAS A\&M & 367 & 85 & ALABAMA & 207 \\
\hline 29 & PITTSBURGH & 362 & 86 & DELAWARE & 207 \\
\hline 30 & DUKE & 361 & 87 & SOUTHERN ILLINOIS & 207 \\
\hline 31 & UTAH & 356 & 88 & DARTMOUTH & 206 \\
\hline 32 & BRITISH COLUMBIA & 349 & 89 & LOUISIANA STATE & 203 \\
\hline 33 & JOHNS HOPKINS & 345 & 90 & BOSTON COLLEGE & 202 \\
\hline 34 & NORTHWESTERN & 339 & 91 & HOUSTON & 201 \\
\hline 35 & CALIFORNIA, SAN DIEGO & 337 & 92 & WESTERN ONTARIO & 195 \\
\hline 36 & ALBERTA & 324 & 93 & LOUISVILLE & 185 \\
\hline 37 & ARIZONA STATE & 322 & 94 & IOWA STATE & 182 \\
\hline 38 & CHICAGO & 318 & 95 & $\mathrm{OHIO}$ & 179 \\
\hline 39 & ARIZONA & 313 & 96 & MASSACHUSETTS & 177 \\
\hline 40 & WASHINGTON U.-ST. LOUIS & 311 & 97 & WATERLOO & 177 \\
\hline 41 & TEXAS TECH & 306 & 98 & TULANE & 175 \\
\hline 42 & NEW MEXICO & 298 & 99 & CALIFORNIA, RIVERSIDE & 172 \\
\hline 43 & BOSTON & 297 & 100 & HOWARD & 171 \\
\hline 44 & IOWA & 297 & 101 & QUEEN'S & 169 \\
\hline 45 & EMORY & 295 & 102 & WASHINGTON STATE & 169 \\
\hline 46 & GEORGIA & 294 & 103 & MCMASTER & 165 \\
\hline 47 & MICHIGAN STATE & 292 & 104 & VIRGINIA TECH & 162 \\
\hline 48 & KANSAS & 289 & 105 & SUNY-ALBANY & 159 \\
\hline 49 & KENT STATE & 284 & 106 & SUNY-STONY BROOK & 159 \\
\hline 50 & TENNESSEE & 284 & 107 & SASKATCHEWAN & 152 \\
\hline 51 & SOUTH CAROLINA & 277 & 108 & CASE WESTERN RESERVE & 147 \\
\hline 52 & NORTH CAROLINA STATE & 276 & 109 & AUBURN & 140 \\
\hline 53 & CALIFORNIA, DAVIS & 273 & 110 & RICE & 132 \\
\hline 54 & GEORGETOWN & 269 & 111 & GUELPH & 131 \\
\hline 55 & WAYNE STATE & 267 & 112 & GEORGIA TECH & 126 \\
\hline 56 & GEORGE WASHINGTON & 264 & 113 & COLORADO STATE & 123 \\
\hline 57 & NOTRE DAME & 261 & & & \\
\hline
\end{tabular}


RANK ORDER TABLE 19

EXPENDITURES FOR ELECTRONIC MATERIALS

\begin{tabular}{|c|c|c|c|c|c|}
\hline RANK & INSTITUTION & VALUE & RANK & INSTITUTION & VALUE \\
\hline 1 & PENNSYLVANIA STATE & $9,823,592$ & 58 & WISCONSIN & $3,606,560$ \\
\hline 2 & COLUMBIA & $7,704,688$ & 59 & ALABAMA & $3,578,275$ \\
\hline 3 & CINCINNATI & $7,239,964$ & 60 & TULANE & $3,572,303$ \\
\hline 4 & TEXAS A\&M & $7,193,666$ & 61 & WATERLOO & $3,523,184$ \\
\hline 5 & ALBERTA & $7,006,150$ & 62 & CONNECTICUT & $3,504,528$ \\
\hline 6 & BRITISH COLUMBIA & $6,723,530$ & 63 & KENTUCKY & $3,491,559$ \\
\hline 7 & NEW YORK & $6,688,267$ & 64 & ILLINOIS, CHICAGO & $3,455,796$ \\
\hline 8 & JOHNS HOPKINS & $6,490,250$ & 65 & CALIFORNIA, SAN DIEGO & $3,439,242$ \\
\hline 9 & TEXAS & $6,038,544$ & 66 & CALIFORNIA, IRVINE & $3,411,180$ \\
\hline 10 & CHICAGO & $6,021,047$ & 67 & SOUTHERN CALIFORNIA & $3,359,071$ \\
\hline 11 & MCGILL & $6,016,847$ & 68 & BROWN & $3,343,811$ \\
\hline 12 & IOWA STATE & $5,991,018$ & 69 & CALIFORNIA, LOS ANGELES & $3,287,538$ \\
\hline 13 & PITTSBURGH & $5,891,621$ & 70 & ILLINOIS, URBANA & $3,247,049$ \\
\hline 14 & NORTHWESTERN & $5,844,331$ & 71 & MIT & $3,245,325$ \\
\hline 15 & WAYNE STATE & $5,669,076$ & 72 & GUELPH & $3,198,679$ \\
\hline 16 & ARIZONA & $5,560,913$ & 73 & MANITOBA & $3,151,007$ \\
\hline 17 & WESTERN ONTARIO & $5,493,986$ & 74 & GEORGETOWN & $3,114,752$ \\
\hline 18 & GEORGIA & $5,478,944$ & 75 & COLORADO STATE & $3,036,234$ \\
\hline 19 & IOWA & $5,421,137$ & 76 & WASHINGTON STATE & $3,020,713$ \\
\hline 20 & HARVARD & $5,417,996$ & 77 & EMORY & $2,935,205$ \\
\hline 21 & MINNESOTA & $5,414,827$ & 78 & SOUTHERN ILLINOIS & $2,921,467$ \\
\hline 22 & CORNELL & $5,366,442$ & 79 & OKLAHOMA & $2,912,861$ \\
\hline 23 & FLORIDA & $5,355,636$ & 80 & CALIFORNIA, RIVERSIDE & $2,908,692$ \\
\hline 24 & WASHINGTON U.-ST. LOUIS & $5,273,151$ & 81 & SYRACUSE & $2,899,414$ \\
\hline 25 & PENNSYLVANIA & $5,231,082$ & 82 & GEORGE WASHINGTON & $2,894,930$ \\
\hline 26 & COLORADO & $5,179,225$ & 83 & CASE WESTERN RESERVE & $2,866,823$ \\
\hline 27 & OHIO STATE & $5,174,694$ & 84 & $\begin{array}{l}\text { MISSOURI } \\
\text { MIST }\end{array}$ & $2,787,967$ \\
\hline 28 & TEMPLE & $5,121,982$ & 85 & LOUISVILLE & $2,703,346$ \\
\hline 29 & MONTREAL & $5,030,158$ & 86 & MASSACHUSETTS & $2,637,996$ \\
\hline 30 & ARIZONA STATE & $5,010,330$ & 87 & AUBURN & $2,476,161$ \\
\hline 31 & PRINCETON & $4,942,212$ & 88 & HAWAII & $2,471,454$ \\
\hline 32 & DELAWARE & $4,910,639$ & 89 & KANSAS & $2,434,356$ \\
\hline 33 & MICHIGAN STATE & $4,899,965$ & 90 & OHIO & $2,425,985$ \\
\hline 34 & VANDERBILT & $4,728,525$ & 91 & NORTH CAROLINA STATE & $2,405,541$ \\
\hline 35 & YALE & $4,689,354$ & 92 & HOUSTON & $2,378,095$ \\
\hline 36 & SUNY-BUFFALO & $4,677,244$ & 93 & TENNESSEE & $2,355,919$ \\
\hline 37 & YORK & $4,525,542$ & 94 & CALIFORNIA, SANTA BARBARA & $2,248,483$ \\
\hline 38 & QUEEN'S & $4,478,343$ & 95 & NEW MEXICO & $2,205,594$ \\
\hline 39 & DUKE & $4,463,339$ & 96 & SUNY-STONY BROOK & $2,128,413$ \\
\hline 40 & LAVAL & $4,323,571$ & 97 & NEBRASKA & $2,115,486$ \\
\hline 41 & NOTRE DAME & $4,214,924$ & 98 & PURDUE & $2,017,160$ \\
\hline 42 & VIRGINIA TECH & $4,195,140$ & 99 & TEXAS TECH & $2,015,136$ \\
\hline 43 & MCMASTER & $4,170,524$ & 100 & BRIGHAM YOUNG & $1,981,220$ \\
\hline 44 & DARTMOUTH & $4,163,977$ & 101 & OREGON & $1,763,019$ \\
\hline 45 & VIRGINIA & $4,157,333$ & 102 & KENT STATE & $1,732,487$ \\
\hline 46 & INDIANA & $4,135,103$ & 103 & BOSTON & $1,716,862$ \\
\hline 47 & RICE & $4,050,736$ & 104 & ROCHESTER & $1,715,993$ \\
\hline 48 & WASHINGTON & $4,022,052$ & 105 & BOSTON COLLEGE & $1,700,151$ \\
\hline 49 & SASKATCHEWAN & $3,943,918$ & 106 & SUNY-ALBANY & $1,671,207$ \\
\hline 50 & MARYLAND & $3,939,405$ & 107 & LOUISIANA STATE & $1,470,331$ \\
\hline 51 & TORONTO & $3,930,250$ & 108 & UTAH & $1,193,845$ \\
\hline 52 & FLORIDA STATE & $3,880,840$ & 109 & HOWARD & $1,070,931$ \\
\hline 53 & MIAMI & $3,844,486$ & 110 & CALIFORNIA, BERKELEY & UA \\
\hline 54 & NORTH CAROLINA & $3,804,794$ & 111 & GEORGIA TECH & UA \\
\hline 55 & CALIFORNIA, DAVIS & $3,792,873$ & 112 & MICHIGAN & UA \\
\hline 56 & RUTGERS & $3,733,667$ & 113 & OKLAHOMA STATE & UA \\
\hline 57 & SOUTH CAROLINA & $3,679,957$ & & & \\
\hline
\end{tabular}

UA - Unavailable 
RANK ORDER TABLE 20

Electronic Materials as a Percent of Total Library Materials

\begin{tabular}{|c|c|c|c|c|c|}
\hline RANK & INSTITUTION & VALUE & RANK & INSTITUTION & VALUE \\
\hline 1 & WAYNE STATE & 72.57 & 58 & ILLINOIS, CHICAGO & 42.32 \\
\hline 2 & CINCINNATI & 70.92 & 59 & NEW MEXICO & 41.57 \\
\hline 3 & IOWA STATE & 67.38 & 60 & RICE & 41.48 \\
\hline 4 & GUELPH & 63.48 & 61 & NEW YORK & 41.44 \\
\hline 5 & DELAWARE & 61.34 & 62 & VIRGINIA & 41.41 \\
\hline 6 & MCMASTER & 61.07 & 63 & SOUTHERN ILLINOIS & 41.05 \\
\hline 7 & SUNY-BUFFALO & 60.23 & 64 & MIT & 40.80 \\
\hline 8 & WESTERN ONTARIO & 59.88 & 65 & BROWN & 40.36 \\
\hline 9 & TEMPLE & 58.56 & 66 & MINNESOTA & 38.25 \\
\hline 10 & WATERLOO & 57.00 & 67 & PENNSYLVANIA & 38.05 \\
\hline 11 & VIRGINIA TECH & 56.91 & 68 & TEXAS & 37.66 \\
\hline 12 & QUEEN'S & 56.82 & 69 & CORNELL & 37.31 \\
\hline 13 & COLORADO & 55.60 & 70 & KENTUCKY & 36.89 \\
\hline 14 & PENNSYLVANIA STATE & 54.31 & 71 & COLUMBIA & 36.66 \\
\hline 15 & YORK & 53.97 & 72 & CHICAGO & 36.57 \\
\hline 16 & DARTMOUTH & 53.74 & 73 & RUTGERS & 35.19 \\
\hline 17 & WASHINGTON STATE & 53.67 & 74 & OREGON & 34.33 \\
\hline 18 & MCGILL & 52.09 & 75 & HAWAII & 32.47 \\
\hline 19 & ALABAMA & 52.08 & 76 & SUNY-STONY BROOK & 32.29 \\
\hline 20 & SOUTH CAROLINA & 52.08 & 77 & WASHINGTON & 32.07 \\
\hline 21 & TEXAS A\&M & 51.02 & 78 & DUKE & 31.97 \\
\hline 22 & SASKATCHEWAN & 50.80 & 79 & MIAMI & 31.97 \\
\hline 23 & FLORIDA STATE & 50.73 & 80 & SUNY-ALBANY & 31.94 \\
\hline 24 & BRITISH COLUMBIA & 49.72 & 81 & HOWARD & 31.39 \\
\hline 25 & WASHINGTON U.-ST. LOUIS & 49.53 & 82 & WISCONSIN & 31.14 \\
\hline 26 & FLORIDA & 49.49 & 83 & GEORGE WASHINGTON & 30.61 \\
\hline 27 & MICHIGAN STATE & 49.25 & 84 & KANSAS & 30.23 \\
\hline 28 & LAVAL & 49.12 & 85 & NEBRASKA & 30.18 \\
\hline 29 & GEORGIA & 48.84 & 86 & INDIANA & 29.73 \\
\hline 30 & MONTREAL & 48.55 & 87 & LOUISVILLE & 29.52 \\
\hline 31 & COLORADO STATE & 48.46 & 88 & PRINCETON & 29.50 \\
\hline 32 & KENT STATE & 48.08 & 89 & HOUSTON & 29.30 \\
\hline 33 & NORTHWESTERN & 48.04 & 90 & GEORGETOWN & 28.88 \\
\hline 34 & ALBERTA & 48.02 & 91 & NORTH CAROLINA & 27.10 \\
\hline 35 & ARIZONA & 47.68 & 92 & ILLINOIS, URBANA & 26.96 \\
\hline 36 & CALIFORNIA, RIVERSIDE & 47.61 & 93 & NORTH CAROLINA STATE & 26.72 \\
\hline 37 & JOHNS HOPKINS & 47.59 & 94 & SOUTHERN CALIFORNIA & 25.91 \\
\hline 38 & MASSACHUSETTS & 47.28 & 95 & ROCHESTER & 25.55 \\
\hline 39 & MANITOBA & 46.62 & 96 & OKLAHOMA & 24.04 \\
\hline 40 & TULANE & 46.54 & 97 & LOUISIANA STATE & 22.02 \\
\hline 41 & SYRACUSE & 46.02 & 98 & CALIFORNIA, LOS ANGELES & 21.69 \\
\hline 42 & $\mathrm{OHIO}$ & 45.54 & 99 & BRIGHAM YOUNG & 21.37 \\
\hline 43 & CASE WESTERN RESERVE & 45.48 & 100 & BOSTON COLLEGE & 21.23 \\
\hline 44 & MARYLAND & 45.29 & 101 & TEXAS TECH & 21.20 \\
\hline 45 & VANDERBILT & 44.70 & 102 & EMORY & 20.84 \\
\hline 46 & AUBURN & 44.67 & 103 & TENNESSEE & 20.40 \\
\hline 47 & CONNECTICUT & 44.62 & 104 & BOSTON & 19.72 \\
\hline 48 & NOTRE DAME & 44.18 & 105 & HARVARD & 19.25 \\
\hline 49 & CALIFORNIA, DAVIS & 43.80 & 106 & PURDUE & 18.38 \\
\hline 50 & ARIZONA STATE & 43.67 & 107 & TORONTO & 18.32 \\
\hline 51 & CALIFORNIA, IRVINE & 43.65 & 108 & UTAH & 15.94 \\
\hline 52 & CALIFORNIA, SAN DIEGO & 43.41 & 109 & YALE & 13.83 \\
\hline 53 & IOWA & 43.21 & 110 & CALIFORNIA, BERKELEY & UA \\
\hline 54 & PITTSBURGH & 43.20 & 111 & GEORGIA TECH & UA \\
\hline 55 & MISSOURI & 43.14 & 112 & MICHIGAN & UA \\
\hline 56 & CALIFORNIA, SANTA BARBARA & 42.94 & 113 & OKLAHOMA STATE & UA \\
\hline 57 & OHIO STATE & 42.39 & & & \\
\hline
\end{tabular}

UA - Unavailable 
RANK ORDER TABLE 21

EXPENDITURES-FOCUSED INDEX OF ARL UNIVERSITY LIBRARIES, 2005-06

\begin{tabular}{|c|c|c|c|c|c|}
\hline RANK & INSTITUTION & VALUE & RANK & INSTITUTION & VALUE \\
\hline 1 & HARVARD & 5.83 & 58 & KANSAS & -0.31 \\
\hline 2 & YALE & 3.71 & 59 & WAYNE STATE & -0.32 \\
\hline 3 & COLUMBIA & 2.39 & 60 & CALIF., DAVIS & -0.34 \\
\hline 4 & TORONTO & 2.32 & 61 & OKLAHOMA & -0.34 \\
\hline 5 & CALIF., BERKELEY & 2.27 & 62 & SUNY-BUFFALO & -0.36 \\
\hline 6 & CALIF., LOS ANGELES & 1.78 & 63 & KENTUCKY & -0.38 \\
\hline 7 & MICHIGAN & 1.76 & 64 & SOUTH CAROLINA & -0.38 \\
\hline 8 & PENNSYLVANIA STATE & 1.64 & 65 & BOSTON & -0.39 \\
\hline 9 & TEXAS & 1.17 & 66 & YORK & -0.43 \\
\hline 10 & CORNELL & 1.16 & 67 & BROWN & -0.44 \\
\hline 11 & NEW YORK & 1.12 & 68 & COLORADO & -0.45 \\
\hline 12 & WISCONSIN & 1.12 & 69 & MANITOBA & -0.46 \\
\hline 13 & PRINCETON & 1.04 & 70 & NEW MEXICO & -0.47 \\
\hline 14 & WASHINGTON & 0.97 & 71 & LOUISVILLE & -0.48 \\
\hline 15 & MINNESOTA & 0.91 & 72 & TEMPLE & -0.50 \\
\hline 16 & ILLINOIS, URBANA & 0.79 & 73 & ILLINOIS, CHICAGO & -0.52 \\
\hline 17 & NORTH CAROLINA & 0.71 & 74 & BOSTON COLLEGE & -0.53 \\
\hline 18 & DUKE & 0.65 & 75 & CALIF., IRVINE & -0.53 \\
\hline 19 & VIRGINIA & 0.63 & 76 & LAVAL & -0.53 \\
\hline 20 & PENNSYLVANIA & 0.63 & 77 & ROCHESTER & -0.54 \\
\hline 21 & INDIANA & 0.54 & 78 & CALIF., SANTA BARBARA & -0.55 \\
\hline 22 & OHIO STATE & 0.54 & 79 & DARTMOUTH & -0.55 \\
\hline 23 & SOUTHERN CALIFORNIA & 0.53 & 80 & WESTERN ONTARIO & -0.56 \\
\hline 24 & CHICAGO & 0.49 & 81 & HAWAII & -0.57 \\
\hline 25 & EMORY & 0.43 & 82 & IOWA STATE & -0.58 \\
\hline 26 & WASHINGTON U.-ST. LOUIS & 0.43 & 83 & DELAWARE & -0.58 \\
\hline 27 & ALBERTA & 0.40 & 84 & HOUSTON & -0.59 \\
\hline 28 & RUTGERS & 0.40 & 85 & RICE & -0.62 \\
\hline 29 & BRITISH COLUMBIA & 0.40 & 86 & QUEEN'S & -0.65 \\
\hline 30 & TEXAS A\&M & 0.36 & 87 & NEBRASKA & -0.66 \\
\hline 31 & PITTSBURGH & 0.31 & 88 & SYRACUSE & -0.68 \\
\hline 32 & JOHNS HOPKINS & 0.24 & 89 & FLORIDA STATE & -0.71 \\
\hline 33 & ARIZONA & 0.22 & 90 & SASKATCHEWAN & -0.73 \\
\hline 34 & MONTREAL & 0.20 & 91 & ALABAMA & -0.74 \\
\hline 35 & FLORIDA & 0.16 & 92 & CASE WESTERN RESERVE & -0.75 \\
\hline 36 & NORTHWESTERN & 0.13 & 93 & CALIF., RIVERSIDE & -0.75 \\
\hline 37 & NORTH CAROLINA STATE & 0.08 & 94 & SOUTHERN ILLINOIS & -0.76 \\
\hline 38 & IOWA & 0.06 & 95 & SUNY-STONY BROOK & -0.76 \\
\hline 39 & MCGILL & 0.05 & 96 & TULANE & -0.77 \\
\hline 40 & ARIZONA STATE & 0.04 & 97 & MASSACHUSETTS & -0.77 \\
\hline 41 & CALIF., SAN DIEGO & 0.02 & 98 & MISSOURI & -0.77 \\
\hline 42 & PURDUE & -0.07 & 99 & WATERLOO & -0.77 \\
\hline 43 & BRIGHAM YOUNG & -0.08 & 100 & VIRGINIA TECH & -0.80 \\
\hline 44 & CONNECTICUT & -0.08 & 101 & OKLAHOMA STATE & -0.80 \\
\hline 45 & GEORGETOWN & -0.08 & 102 & MCMASTER & -0.84 \\
\hline 46 & MICHIGAN STATE & -0.09 & 103 & OREGON & -0.85 \\
\hline 47 & MIAMI & -0.10 & 104 & OHIO & -0.85 \\
\hline 48 & MARYLAND & -0.11 & 105 & COLORADO STATE & -0.85 \\
\hline 49 & CINCINNATI & -0.11 & 106 & WASHINGTON STATE & -0.85 \\
\hline 50 & TENNESSEE & -0.13 & 107 & LOUISIANA STATE & -0.87 \\
\hline 51 & VANDERBILT & -0.13 & 108 & AUBURN & -0.91 \\
\hline 52 & GEORGIA & -0.15 & 109 & SUNY-ALBANY & -0.94 \\
\hline 53 & UTAH & -0.20 & 110 & GEORGIA TECH & -0.98 \\
\hline 54 & GEORGE WASHINGTON & -0.24 & 111 & GUELPH & -1.00 \\
\hline 55 & MIT & -0.25 & 112 & KENT STATE & -1.06 \\
\hline 56 & NOTRE DAME & -0.25 & 113 & HOWARD & -1.12 \\
\hline 57 & TEXAS TECH & -0.27 & & & \\
\hline
\end{tabular}



ARL STATISTICS QUESTIONNAIRE AND INSTRUCTIONS, 2005-06 


\section{ARL STATISTICS QUESTIONNAIRE, 2005-06 INSTRUCTIONS FOR COMPLETING THE QUESTIONNAIRE}

\section{$\underline{\text { General Instructions }}$}

Definitions of statistical categories can be found in NISO Z39.7-2004, Information Services and Use: Metrics \& statistics for libraries and information providers--Data Dictionary (http://www.niso.org/emetrics/current/index.html). ARL has been modifying the interpretation of the standard definitions to address questions posed by by the library staff at various member institutions that complete the survey and with feedback from the ARL Statistics and Assessment Committee (http://www.arl.org/stats/program/meeting.html).

Please do not use decimals. All figures should be rounded to the nearest whole number.

Please respond to every question. If an exact figure cannot be provided, use NA/UA to indicate that the figure is either unavailable or not applicable. If the appropriate answer is zero or none, use $\mathbf{0 .}$

Although the form allows for data to be entered from both main and branch campuses, an effort should be made to report figures for the main campus only. (The U.S. National Center for Education Statistics, Integrated Postsecondary Education Data System (IPEDS) defines a branch institution as "a campus or site of an educational institution that is not temporary, is located in a community beyond a reasonable commuting distance from its parent institution, and offers organized programs of study, not just courses.”) If figures for libraries located at branch campuses are reported, please specify which branch libraries are included and which ones are excluded in the notes below.

A branch library is defined as an auxiliary library service outlet with quarters separate from the central library of an institution, which has a basic collection of books and other materials, a regular staffing level, and an established schedule. A branch library is administered either by the central library or (as in the case of some law and medical libraries) through the administrative structure of other units within the university. Departmental study/reading rooms are not included.

The questionnaire assumes a fiscal year ending June 30, 2006. If your fiscal year is different, please indicate this in the notes below by adjusting the reporting period.

Footnotes. Explanatory footnotes will be included with the published statistics. Provide any notes you may have in the footnotes area at the end of the survey. Reporting libraries are urged to record there any information that would clarify the figures submitted in that line, e.g., the inclusion and exclusion of branch campus libraries. Please make an effort to word your footnotes in a manner consistent with notes appearing in the published report, so that the ARL Office can interpret your footnotes correctly.

\section{$\underline{\text { Specific Instructions }}$}

Question 1. Volumes in Library. Use the ANSI/NISO Z39.7-2004 definition for volume as follows:

a single physical unit of any printed, typewritten, handwritten, mimeographed, or processed work, distinguished from other units by a separate binding, encasement, portfolio, or other clear distinction, which has been cataloged, classified, and made ready for use, and which is typically the unit used to charge circulation transactions. Either a serial volume is bound, or it comprises the serial issues that would be bound together if the library bound all serials.

Include duplicates and bound volumes of periodicals. For purposes of this questionnaire, unclassified bound serials arranged in alphabetical order are considered classified. Exclude microforms, maps, nonprint materials, and uncataloged items. If any of these items cannot be excluded, please provide an explanatory footnote 
Include government document volumes that are accessible through the library's catalogs regardless of whether they are separately shelved. "Classified" includes documents arranged by Superintendent of Documents, CODOC, or similar numbers. "Cataloged" includes documents for which records are provided by the library or downloaded from other sources into the library's card or online catalogs. Documents should, to the extent possible, be counted as they would if they were in bound volumes (e.g., 12 issues of an annual serial would be one or two volumes). Title and piece counts should not be considered the same as volume counts. If a volume count has not been kept, it may be estimated through sampling a representative group of title records and determining the corresponding number of volumes, then extrapolating to the rest of the collection. As an alternative, an estimate may be made using the following formulae:

\section{2 documents pieces per foot \\ 10 "traditional" volumes per foot \\ 5.2 documents pieces per volume}

Include e-book units, as long as these e-books have been purchased and are owned by your library. If you have access to netLibrary titles as a result of participating in various consortia, do not report these e-books as your library's property unless the e-books actually belong to your library. If the books were purchased by a consortium, they may belong to the consortium itself and not to the participating libraries. Report only the number of e-books that belong to your library and are cataloged, classified and made ready for use. Provide a footnote explaining how many e-books you are reporting, preferably by specifying the products and the number of titles in a note.

For information on how to count items housed in remote storage, see the Interim Guidelines for Counting Materials Housed in Library Storage Centers, at http://www.arl.org/stats/arlstat/storage.html .

If either formulas or sampling are used for deriving your count, please indicate in a footnote.

Question 1b. Volumes Added. Include only volumes cataloged, classified, and made ready for use. Include government documents if they have been included in the count of volumes on line 1a. Do not include as part of Volumes Added Gross any government documents or other collections (such as large gift collections or e-book packages) that were added to the collection as the result of a one time download or addition to the OPAC. Include these items in Volumes Held of the previous year and provide a footnote explaining the revision of Line 1a.

Include e-book units, as long as these e-books have been purchased and are owned by your library. If you have access to netLibrary titles as a result of participating in various consortia, do not report these e-books as your library's property unless the e-books actually belong to your library. If the books were purchased by a consortium, they may belong to the consortium itself and not to the participating libraries. Report only the number of e-books that belong to your library and are cataloged, classified and made ready for use. Provide a footnote explaining how many e-books you are reporting, preferably by specifying the products and the number of titles in a note.

Question 2. Monographic Volumes Purchased. Report number of volumes purchased; do not include volumes received or cataloged. Include all volumes for which an expenditure was made during 2005-06, including volumes paid for in advance but not received during the fiscal year. Include monographs in series and continuations. Include e-books that fit the netLibrary model, i.e., electronic manifestations of physical entities and/or units; provide a footnote explaining how many e-books you are reporting, preferably by specifying the products and the number of titles. If only number of titles purchased can be reported, please report the data and provide an explanatory footnote.

Question 3: Basis of Volume Count. A physical count is a piece count; a bibliographic count is a catalog record count.

Questions 4-5. Serials. Report the total number of subscriptions, not titles. Include duplicate subscriptions and, to the extent possible, all government document serials even if housed in a separate documents collection. Verify the inclusion or exclusion of document serials in Question 5. Exclude unnumbered monographic and publishers' series. Electronic serials acquired as part of an aggregated package (e.g., Project MUSE, BioOne, ScienceDirect) should be counted by title. A serial is 
a publication in any medium issued in successive parts bearing numerical or chronological designations and intended to be continued indefinitely. This definition includes periodicals, newspapers, and annuals (reports, yearbooks, etc.); the journals, memoirs, proceedings, transactions, etc. of societies; and numbered monographic series.

In the case of consortial agreements, count under Q4a only those subscriptions to titles for which the library pays directly from its budgeted expenditures reported under Q15b (expenditures for serials). Count under Q4a only those titles and subscriptions for which your library pays. Report other subscriptions that your library receives and does not pay for directly under Q4b (serials received and not purchased). If a purchased subscription includes electronic access to the title, count that subscription twice: once for the print version and once for the electronic version. If serials have been purchased through a consortium whose budget is centrally funded and independent from the library's budget, these serials should be reported under Q4b.

Do not include the full-text serials from such indexing/abstracting products as Wilson Social Sciences Abstracts Full Text, Lexis-Nexis, ABI/INFORM, and other indexes with access to the full text of articles. These full-text titles are counted in in the ARL Supplementary Statistics.

Question 4b. Serials: Not Purchased. Record those serials whose subscriptions were received without purchase for whatever reason. If separate counts of nonpurchased and purchased serials are not available, report only the total number of current serials received on line 4, and report U/A for lines 4a and 4b.

Question 6. Microforms. Report the total number of physical units: reels of microfilm, microcards, and microprint and microfiche sheets. Include all government documents in microform; provide a footnote if documents are excluded.

Question 7. Government documents. Report the total number of physical units (pieces) of government documents in paper format that have not been counted elsewhere. Include local, state, national, and international documents; include documents purchased from a commercial source if shelved with separate documents collections and not counted above. Include serials and monographs. To estimate pieces from a measurement of linear feet, use the formula 1 foot $=52$ pieces and indicate in a footnote that the count is based on this estimate. Exclude microforms and nonprint formats such as maps or CD-ROMs. Adjust line 1a, i.e., last year's Volumes Held, and provide a footnote if you are adding records to the OPAC for government documents previously held but not counted as part of Volumes Held (line 1a).

Question 8. Computer files. Include the number of pieces of computer-readable disks, tapes, CD-ROMs, and similar machine-readable files comprising data or programs that are locally held as part of the library's collections available to library clients. Examples are U.S. Census data tapes, sample research software, locally-mounted databases, and reference tools on CD-ROM, tape or disk. Exclude bibliographic records used to manage the collection (i.e., the library's own catalog in machine-readable form), library system software, and microcomputer software used only by the library staff.

Question 9. Manuscripts and archives. Include both manuscripts and archives measured in linear feet.

Question 10. Cartographic materials. Include the numbers of pieces of two- and three-dimensional maps and globes. Include satellite and aerial photographs and images.

Question 11. Graphic materials. Include the number of pieces of prints, pictures, photographs, postcards, slides, transparencies, film strips, and the like.

Question 12. Audio materials. Include the number of pieces of audiocassettes, phonodiscs, audio compact discs, reel-to-reel tapes, and other sound recordings.

Question 13. Film and video materials. Include the number of pieces of motion pictures, videocassettes, video laser discs, and similar visual materials. 
Questions 14-20. Expenditures. Report all expenditures of funds that come to the library from the regular institutional budget, and from sources such as research grants, special projects, gifts and endowments, and fees for service. (For question 17, include non-library funds; see instruction Q17.) Do not report encumbrances of funds that have not yet been expended. Canadian libraries should report expenditures in Canadian dollars. (For your information, if interested in determining figures in U.S. dollars, divide Canadian dollar amounts by 1.16289, the average monthly noon exchange rate published in the Bank of Canada Review for the period July 2005-June 2006.) Please round figures to the nearest dollar.

Question 15a. Monographs. Report expenditures for volumes counted on line 2.

Question 15b. Current Serials. Report expenditures for serials counted on line 4a. Exclude unnumbered monographic and publishers' series, and encumbrances.

Question 15c. Other library materials. Include expenditures for all materials not reported in Questions 15a and 15b, e.g., backfiles of serials, charts and maps, audiovisual materials, manuscripts, etc. If expenditures for these materials are included in lines 15a and/or 15b and cannot be disaggregated, please report U/A and provide a footnote. Do not include encumbrances.

Question 15d. Miscellaneous expenditures. Include any other materials funds expenditures not included in questions 15a-c, e.g., expenditures for bibliographic utilities, literature searching, security devices, memberships for the purposes of publications, etc. Please list categories, with amounts, in a footnote. Note: If your library does not use materials funds for non-materials expenditures-i.e., such expenditures are included in "Other Operating Expenditures" - report 0, not U/A, on line 15d.

Question 16. Contract Binding. Include only contract expenditures for binding done outside the library. If all binding is done in-house, state this fact and give in-house expenditures in a footnote; do not include personnel expenditures. (This figure should also be reported in the 2005-06 ARL Preservation Survey, question 7b.)

Questions 17. Salaries and wages. Exclude fringe benefits. If professional and support staff salaries cannot be separated, enter $\mathbf{U} / \mathbf{A}$, on lines $17 \mathrm{a}$ and $17 \mathrm{~b}$ and enter total staff on line 17.

Question 17c. Salaries and wages: Student Assistants. Report $100 \%$ of student wages regardless of budgetary source of funds. Include federal and local funds for work study students.

Question 19. Other operating expenditures. Exclude expenditures for buildings, maintenance, and fringe benefits.

Questions 21-25. Electronic materials expenditures. These items are intended to indicate what portion of your institution's total library expenditures are dedicated to electronic resources and services. Please use the Footnotes to indicate any electronic materials expenditures you believe not to be covered by these questions. Many expenditures recorded in these questions should have been included in Question 20, total library expenditures.

Question 21. Computer files. Report expenditures that are not current serials (i.e. are non-subscription, one-time, or monographic in nature) for software and machine-readable materials considered part of the collections. Examples include periodical backfiles, literature collections, one-time costs for JSTOR membership, etc. Expenditures reported here may be derived from any of the following categories: Monographs (Q15a), Other Library Materials (Q15c), Miscellaneous (Q15d), or Other Operating Expenditures (Q19).

Question 22. Electronic Serials. Report subscription expenditures (or those which are expected to be ongoing commitments) for serial publications whose primary format is electronic and for online searches of remote databases such as OCLC FirstSearch, DIALOG, Lexis-Nexis, etc. Examples include paid subscriptions for electronic journals and indexes/abstracts available via the Internet, CD-ROM serials, and annual access fees for resources purchased on a "one-time" basis, such as literature collections, JSTOR membership, etc. Not all items whose expenditures are counted here will be included in Total Current Serials (Question 4) or Current Serial Expenditures (Question 15b). 
Q23. Bibliographic Utilities, Networks, and Consortia. Because it is increasingly common for ARL Libraries to enter into consortial arrangements to purchase access to electronic resources, both "Library" and "External" expenditure blanks and instructions are provided. Please use afootnote to describe expenditures that you believe are not covered by the question, or situations that do not seem to fit the instructions.

Q23a. From internal library sources. Report expenditures paid by the Library for services provided by national, regional, and local bibliographic utilities, networks, and consortia, such as OCLC and RLG, unless for user database access and subscriptions, which should be reported in Questions 21 or 22. Include only expenditures that are part of Other Operating Expenditures (Q19).

Q23b. From external sources. If your library receives access to computer files, electronic serials or search services through one or more centrally-funded system or consortial arrangements for which it does not pay fully and/or directly (for example, funding is provided by the state on behalf of all members), enter the amount paid by external bodies on its behalf. If the specific dollar amount is not known, but the total student FTE for the consortium and amount spent for the academic members are known, divide the overall amount spent by your institution's share of the total student FTE.

Q24. Computer hardware and software. Report expenditures from the library budget for computer hardware and software used to support library operations, whether purchased or leased, mainframe or microcomputer, and whether for staff or public use. Include expenditures for: maintenance; equipment used to run information service products when those expenditures can be separated from the price of the product; telecommunications infrastructure costs, such as wiring, hubs, routers, etc. Include only expenditures that are part of Other Operating Expenditures (Q19).

Q25. Document Delivery/Interlibrary Loan. Report expenditures for document delivery and interlibrary loan services (both borrowing and lending). Include fees paid for photocopies, costs of telefacsimile transmission, royalties and access fees paid to provide document delivery or interlibrary loan. Include fees paid to bibliographic utilities if the portion paid for interlibrary loan can be separately counted. Include only expenditures that are part of Miscellaneous Materials Expenditures (Q15d) or Other Operating Expenditures (Q19), and only for those ILL/DD programs with data recorded in Questions 34-35.

Questions 26. Personnel. Report the number of staff in filled positions, or positions that are only temporarily vacant. ARL defines temporarily vacant positions as positions that were vacated during the fiscal year for which ARL data were submitted, for which there is a firm intent to refill, and for which there are expenditures for salaries reported on line 17.

Include cost recovery positions and staff hired for special projects and grants, but provide an explanatory footnote indicating the number of such staff. If such staff cannot be included, provide a footnote. To compute full-time equivalents of part-time employees and student assistants, take the total number of hours per week (or year) worked by part-time employees in each category and divide it by the number of hours considered by the reporting library to be a full-time work week (or year). Round figures to the nearest whole numbers.

Question 26a. Professional Staff. Since the criteria for determining professional status vary among libraries, there is no attempt to define the term "professional." Each library should report those staff members it considers professional, including, when appropriate, staff who are not librarians in the strict sense of the term, for example computer experts, systems analysts, or budget officers.

Question 26c. Student Assistants. Report the total FTE (see instruction Q26) of student assistants employed on an hourly basis whose wages are paid from funds under library control or from a budget other than the library's, including federal work-study programs. Exclude maintenance and custodial staff.

Question 27. Number of staffed library service points. Count the number of staffed public service points in the main library and in all branch libraries reported in this inventory, including reference desks, information desks, circulation, current periodicals, reserve rooms, reprographic services (if staffed as a public facility), etc. Report the number of designated locations, not the number of staff. 
Question 28. Number of weekly public service hours. Report an unduplicated count of the total public service hours per typical full-service week (i.e., no holidays or other special accommodations) across both main library and branches using the following method (corresponds to IPEDS): If a library is open from 9:00 a.m. to 5:00 p.m. Monday through Friday, it should report 40 hours per week. If several of its branches are also open during these hours, the figure remains 40 hours per week. Should Branch A also be open one evening from 7:00 p.m. to 9:00 p.m., the total hours during which users can find service somewhere within the system becomes 42 hours per week. If Branch B is open the same hours on the same evening, the count is still 42, but if Branch B is open two hours on another evening, or remains open two hours later, the total is then 44 hours per week. Exclude 24-hour unstaffed reserve or similar reading rooms. The maximum total is 168 (i.e., a staffed reading room open 7 days per week, 24 hours per day).

Questions 29-30. Instruction. Sampling based on a typical week may be used to extrapolate TO A FULL YEAR for Questions 29 and 30. Please indicate if responses are based on sampling.

Question 29. Presentations to Groups. Report the total number of sessions during the year of presentations made as part of formal bibliographic instruction programs and through other planned class presentations, orientation sessions, and tours. If the library sponsors multi-session or credit courses that meet several times over the course of a semester, each session should be counted. Presentations to groups may be for either bibliographic instruction, cultural, recreational, or educational purposes. Presentations both on and off the premises should be included as long as they are sponsored by the library. Do not include meetings sponsored by other groups using library meeting rooms. Do not include training for library staff; the purpose of this question is to capture information about the services the library provides for its clientele. Please indicate if the figure is based on sampling.

Question 30. Participants in Group Presentations. Report the total number of participants in the presentations reported on line 29. For multi-session classes with a constant enrollment, count each person only once. Personal, one-to-one instruction in the use of sources should be counted as reference transactions on line 31. Please indicate if the figure is based on sampling. Use a footnote to describe any special situations.

Question 31. Reference Transactions. Report the total number of reference transactions. A reference transaction is

an information contact that involves the knowledge, use, recommendations, interpretation, or instruction in the use of one or more information sources by a member of the library staff. The term includes information and referral service. Information sources include (a) printed and nonprinted material; (b) machinereadable databases (including computer-assisted instruction); (c) the library's own catalogs and other holdings records; (d) other libraries and institutions through communication or referral; and (e) persons both inside and outside the library. When a staff member uses information gained from previous use of information sources to answer a question, the transaction is reported as a reference transaction even if the source is not consulted again.

If a contact includes both reference and directional services, it should be reported as one reference transaction. Duration should not be an element in determining whether a transaction is a reference transaction. Sampling based on a typical week may be used to extrapolate TO A FULL YEAR for Question 31. Please indicate if the figure is based on sampling.

EXCLUDE SIMPLE DIRECTIONAL QUESTIONS. A directional transaction is an information contact that facilitates the logistical use of the library and that does not involve the knowledge, use, recommendations, interpretation, or instruction in the use of any information sources other than those that describe the library, such as schedules, floor plans, and handbooks.

Questions 32-33. Circulation. For Question 32, count the number of initial circulations during the fiscal year from the general collection for use usually (although not always) outside the library. Do not count renewals. Include circulations to and from remote storage facilities for library users (i.e., do not include transactions reflecting transfers or stages of technical processing). Count the total number of items lent, not the number of borrowers. For 
Question 33, report total circulation for the fiscal year including initial transactions reported on line 32 and renewal transactions. Exclude reserve circulations; these are no longer reported.

Questions 34-35. Interlibrary Loans. Report the number of requests for material (both returnables and nonreturnables) provided to other libraries on line 34 and the number of filled requests received from other libraries or providers on line 35. On both lines, include originals, photocopies, and materials sent by telefacsimile or other forms of electronic transmission. Include patron-initiated transactions. Exclude requests for materials locally owned and available on the shelves or electronically. Do not include transactions between libraries covered by this questionnaire.

Questions 36. Ph.D. Degrees. Report the number awarded during the 2004-05 fiscal year. Please note that only the number of Ph.D. degrees are to be counted. Statistics on all other advanced degrees (e.g., D.Ed., D.P.A., M.D., J.D.) should not be reported in this survey. If you are unable to provide a figure for Ph.D.s only, please add a footnote.

Question 37. Ph.D. Fields. For the purposes of this report, Ph.D. fields are defined as the specific discipline specialties enumerated in the U.S. Department of Education's Integrated Postsecondary Education Data System (IPEDS) "Completions" Survey. Although the IPEDS form requests figures for all doctoral degrees, only fields in which Ph.D.s are awarded should be reported on the ARL questionnaire. Any exceptions should be footnoted.

Question 38. Instructional Faculty. Instructional faculty are defined by the U.S. Dept. of Education as

members of the instruction/research staff who are employed full-time as defined by the institution, including faculty with released time for research and faculty on sabbatical leave.

Full-time counts generally exclude faculty who are employed to teach fewer than two semesters, three quarters, two trimesters, or two four-month sessions; replacements for faculty on sabbatical leave or leave without pay; faculty for preclinical and clinical medicine; faculty who are donating their services; faculty who are members of military organizations and paid on a different pay scale from civilian employees; academic officers, whose primary duties are administrative; and graduate students who assist in the instruction of courses. Please be sure the number reported, and the basis for counting, are consistent with those for 2004-05 (unless in previous years faculty were counted who should have been excluded according to the above definition). Please footnote any discrepancies.

Questions 39-42. Enrollment. U.S. libraries should use the Fall 2005 enrollment figures reported to the Department of Education on the Integrated Postsecondary Education Data System survey. Please check these figures against the enrollment figures reported to ARL last year to ensure consistency and accuracy. Note: In the past, the number of part-time students reported was FTE; the number now reported to IPEDS is a head count of parttime students. Canadian libraries should note that the category "graduate students" as reported here includes all post-baccalaureate students.

\section{FOOTNOTES}

Please consult the printed copy of the ARL Statistics 2004-05 for a copy of last year's footnotes. A pdf version is available at: http://www.arl.org/stats/arlstat/. Explanatory footnotes will be included with the published statistics. Reporting libraries are urged to record in the footnote section any information that would clarify the figures submitted, e.g., the inclusion and exclusion of branch campus libraries (see the "General Instructions" for definition of branch campus libraries). Please make an effort to word your footnotes in a manner consistent with notes appearing in the published report, so that the ARL Office can interpret your footnotes correctly.

Submit the completed questionnaire by January 30, 2006.

For assistance, please e-mail Martha Kyrillidou (martha@arl.org) or Mark Young (tatsra@arl.org)

Tel. (202) 296-2296. 


\section{ARL STATISTICS 2005-06 WORKSHEET}

This worksheet is designed to help you plan your submission for the 2005-06 ARL Statistics. The figures on this worksheet should be similar to those in the "Summary" page of your web form, except in cases where data are unavailable. If an exact figure is unavailable, use "NA/UA". If the appropriate answer is zero or none, use " 0. ."

Reporting Institution Date Returned to ARL

Report Prepared by (name)

Title

Email address Phone number

Contact person (if different)

Title

Email address Phone number

\section{PAGE ONE - VOLUMES}

1. Volumes held June 30, 2006

1a. Volumes held June 30, 2005

1b. Volumes added during the year

(i) Volumes added - Gross

(1.b.i)

(ii) Volumes withdrawn during year

(1.b.ii)

(Net Volumes Added: 1.b.i-1.b.ii)

(1.b)

(Volumes held June 30, 2006: 1.a+ 1.b)

2. Number of monographic volumes purchased

3. Basis of volume count is:

(3) Physical Bibliographic 
PAGE TWO - OTHER COLLECTIONS

SERIALS

4. Total number of current serials received, including periodicals

4a. Number of current serials purchased

4b. Number of current serials received but not purchased (Exchanges, gifts, deposits, etc. See instructions.)

(Total serials received: $4 . a+4 . b)$

5. Government documents are included in count of Current Serials?

\section{OTHER LIBRARY MATERIALS}

6. Microform units

(6)

7. Government documents not counted elsewhere

(7)

8. Computer files

(8)

9. Manuscripts and archives (linear ft.) (4a)

(4b)

(4)

(5) Yes No

\section{AUDIOVISUAL MATERIALS}

10. Cartographic

11. Graphic

12. Audio

13. Film and Video 
14. Are the below figures reported in Canadian dollars?

15. Total Library Materials Expenditures

15a. Monographs

15b. Current serials, including periodicals

15c. Other Library Materials

15d. Miscellaneous

(Total library materials: $15 . a+15 . b+15 . c+15 . d$ )
(14)

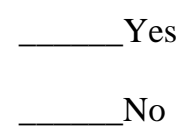

16. Contract binding

(15a)

(15b)

(15c)

(15d)

(15)

(16)

\section{Total Salaries and Wages}

17a. Professional staff

17b. Support staff

17c. Student assistants

(Total salaries and wages: $17 . a+17 . b+17 . c)$ (17a)

18. Fringe benefits are included in expenditures for salaries and wages?

(17)
(18) Yes No

19. Other operating expenditures

20. Total library expenditures

$$
(15+16+17+19)
$$

\section{ELECTRONIC MATERIALS EXPENDITURES}

21. Computer files (One-time/monographic purchases.)

22. Electronic serials

23. Bibliograhpic Utilities, Networks, and Consortia

23a. From internal library sources

23b. From external sources

24. Computer hardware and software

25. Document Delivery/Interlibrary Loan 
PAGE FOUR - PERSONNEL AND PUBLIC SERVICES

PERSONNEL (Round figures to nearest whole number.)

\section{Total Staff FTE}

26a. Professional staff

26b. Support staff

26c. Student assistants

(Total staff FTE: $26 . a+26 . b+26 . c)$ (26a)

(26b)

(26c)

(26)

\section{STAFFED SERVICE POINTS AND HOURS}

27. Number of staffed library service points

28. Number of weekly public service hours

\section{INSTRUCTION}

29. Number of library presentations to groups

29a. Figure based on sampling?

(29a)

Yes _ No

30. Number of total participants in group presentations reported in line 29

30a. Figure based on sampling?

(30a)

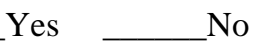

\section{REFERENCE}

31. Number of reference transactions

31a. Figure based on sampling?
(31)

(31a) Yes No 
PAGE FIVE - PUBLIC SERVICES AND LOCAL CHARACTERISTICS

\section{CIRCULATION}

32. Number of initial circulations (excluding reserves)

33. Total circulations (initial and renewals, excluding reserves)

\section{INTERLIBRARY LOANS}

34. Total number of filled requests provided to other libraries

35. Total number of filled requests received from other libraries or providers

\section{Ph.D. DEGREES AND FACULTY}

36. Number of Ph.D.s awarded in FY2005-06

37. Number of fields in which Ph.D.s can be awarded

38. Number of full-time instructional faculty in FY2005-06

ENROLLMENT - FALL 2005

(Line numbers refer to IPEDS survey form.)

39. Full-time students, undergraduate and graduate (Add line 8, columns 15 \& 16, and line 14, columns 15 \& 16.)

40. Part-time students, undergraduate and graduate (Add line 22, columns $15 \& 16$, and line 28, columns 15 \& 16.)

41. Full-time graduate students (Line 14 , columns 15 \& 16.)

42. Part-time graduate students (Line 28 , columns 15 \& 16.)

\section{FOOTNOTES}

On the web form, you will be able to add footnotes to individual questions, as well as footnotes that apply to your entire institution. Please provide any information which would clarify the figures submitted, e.g., the inclusion of branch campus libraries or any special projects which might cause radical increases or decreases from the 2004-05 data. The form will not be able to provide all of your institution's 2004-05 footnotes; please use the footnotes published in the ARL Statistics 2004-05 for comparison if necessary. Please make an effort to word your footnotes in a manner consistent with notes appearing in the published report, so that the ARL Office can interpret your footnotes correctly.

Submit the completed questionnaire by January 30, 2007.

For assistance, please e-mail Martha Kyrillidou (martha@arl.org) or Mark Young (statsra@arl.org)

Tel. (202) 296-2296. 



\section{FOOTNOTES TO THE ARL STATISTICS, 2005-06}

Footnotes may also include errata and corrections to data from prior years not previously reported. Numbers in parentheses refer to columns in Library Data Tables and to Questionnaire numbers.

\section{ALABAMA}

Volumes held June 30, 2005 revised to 2,518,200 due to a mistake from the Map Library in 2004-05.

15.d Includes the Center for Research Libraries membership.

19 Includes bibliographic utilities, memberships (except CRL), and literature searching.

21 Includes purchase of e-books and $1 \mathrm{x}$ full-text collections; increase due to purchase of several major 1x full-text collections (e.g., Eighteenth Century Collections Online).

22 Increase due to continued moving of print plus electronic to electronic-only subscriptions.

\section{ALBERTA}

All figures are as of March 31, 2006.

15-25 Expenditures as reported in Canadian dollars: (15a) $\$ 6,350,436$; (15b) $\$ 9,875,546 ;(15 c) \$ 0 ;(15 d) \$ 741,146 ;(15)$

$\$ 16,967,128$; (16) \$145,733; (17a) \$5,994,083; (17b) \$9,130,644; (17c) \$933,897; (17) \$16,058,624; (19) \$2,399,262; (20)

$\$ 35,570,747$; (21) \$980,313; (22) \$7,167,069; (23a) UA/NA; (23b) UA/NA; (24) UA/NA; (25) UA/NA.

\section{ARIZONA}

Volumes held June 30, 2005 revised to 5,517,564 to reflect the inclusion of new collections of electronic monographs not previously cataloged.

\section{ARIZONA STATE}

Volumes held June 30, 2005 revised to 4,178,244 due to the following e-book record loads completed in 2005-06: Early English Books Online (13,142 additional titles loaded); Ebrary (4,703 additional titles loaded); Knovel (152 additional titles loaded); MyiLibrary (3,370 titles in new e-book package purchased and loaded in 2005-06); netLibrary (145 additional titles purchased and loaded in 2005-06).

2 Includes the following e-book subscription purchases: Knovel (779 titles), Lippincott Springhouse Nursing Collection (12 titles), Lippincott Clinical Choice Collection (42 titles), MIT CogNet (447 titles), netLibrary (20 additional individual titles purchased), Safari Tech Books Online (603 titles), SourceOECD (Books) (2,554 volumes), Stanford Encyclopedia of Philosophy (1 title), Stat!Ref Electronic Medical Library (31 titles), Teatro Espanol del Siglo de Oro (848 plays), Waterloo Directory of English Newspapers \& Periodicals 1800-1900, Series 2 (20 volumes), and Zaha Hadid: Works (Planet Architecture Series) (1 title).

15.d Includes: Conservation/Preservation $(\$ 14,604)$, Memberships $(\$ 101,003)$, ILL/Document Delivery $(\$ 34,684)$, and uncataloged monographs purchased for staff use (\$717).

\section{AUBURN}

All figures are as of September 30, 2006. Volumes held June 30, 2005 revised to 2,955,859.

1.b Several retrospective conversion projects were completed.

2 Decrease due to approval vendors supplying fewer volumes.

$4 \mathrm{~b}$ Decrease due to a large drop in government document serials.

7 2004-05 figure was incorrect.

12 Includes additional titles added from Naxos.

15.b Includes serial databases that were counted in 2004-05 as Other Library Materials (15c).

37 Four (4) new Ph.D. fields were added.

41 2004-05 figure was incorrectly computed and should be revised to 1,560.

\section{BOSTON}

23.b Includes the Theology Library only.

30.a Mugar figure is based on sampling $(4,865)$, Theology and Special Collections data not based on sampling.

31.a Mugar and Special Collections data were not based on sampling, the Theology figure (425) was. 


\section{BOSTON COLLEGE}

Volumes held June 30, 2005 revised to 2,371,139 to include 246,897 e-books.

8 Figure is better tracked due to better accounting.

15.d Includes: Physical processing of shelf ready $(\$ 10,556)$, Boston Library Consortium delivery fee $(\$ 8,438)$, Portico and LOCKSS $(\$ 20,550)$, and miscellaneous $(\$ 50)$.

\section{BOSTON PUBLIC}

Volumes held June 30, 2005 revised to 7,443,759.

2 Increase from 2004-05 is due to purchases that overlapped between the two fiscal years which were not counted in 2004-05.

\section{BRIGHAM YOUNG}

All figures are for the calendar year ending December 31, 2005.

1 Includes 146,551 e-books as indicated in a bibliographic report. All other volumes are a physical count of items.

2 Includes 49,766 e-books added during 2005 to the catalog.

8 This item was discontinued at both the Main and Law libraries.

\section{BRITISH COLUMBIA}

All figures are as of March 31, 2006 and also include the new UBC Okanagan campus (UBCO) with its own branch library as of July 1, 2005. New collections added and additional expenditures noted in other footnotes. Xwi7xwa Library: included in base volume statistics (changed from RR to Branch). Volumes held June 30, 2005 revised to $5,686,642$ as a result.

1.b.i Increase is due to: A change in methodology of acquisitions (shelf-ready books from Coutts); addition of backlog of gift books; addition of backlog of newly catalogued foreign language titles; addition of new collections added at UBCO campus; addition of e-books purchased and catalogued in 2005-06.

4 Increase is due to: Transition to Online program; inclusion of UBCO campus; purchase of new bundles (packages e.g., SAGE), consortia purchases.

6 Increase is due to: Year-end big purchases; addition of UBCO campus.

8 Decrease due to an error in count in 2004-05.

11, 12 Increases are due to the inclusion of the UBCO campus.

13 Increase is due to the inclusion of the UBCO campus, as well as significant a/v collections.

15-25 Expenditures as reported in Canadian dollars: (15a) \$2,490,029; (15b) \$11,512,277; (15c) \$819,786; (15d) \$904,639; (15) $\$ 15,726,731$; (16) $\$ 199,388$; (17a) \$7,681,298; (17b) \$7,515,179; (17c) \$927,964; (17) \$16,124,441; (19) \$3,325,366; (20) \$35,375,926; (21) \$320,081; (22) \$7,498,645; (23a) \$51,437; (23b) \$0; (24) UA/NA; (25) \$189,055.

15 Includes expenditures at the UBCO campus.

15.a Expenditures for e-books are included in expenditures for e-resources - one-time funds.

15.b Increase is due to: Costs; Transition to Online; inclusion of UBCO campus; new packages; and consortia buys.

15.c Increase is due to the inclusion of the UBCO campus.

17.a-b Includes UBCO.

17.c Decrease in expenditures due to closure of Learning Commons for renovations.

19, 20 Includes UBCO campus.

21 Includes year-end desiderata one-time expenditure.

22 Includes Transition to Online, and consortia packages.

24 Expenditure included in other operating expenditures.

26 Increase due to the inclusion of the UBCO campus.

26a-b Includes new campus UBCO; change of employees' employee group (support staff to management/professional); development staff additions. 
26.c Decrease due to closure of Learning Commons for renovations.

29, 30 Figure reflects inclusion of UBCO campus and outreach programs.

33 Decrease is due to change in ILS system and increase in loan period for graduate students (thus, fewer renewals).

34 Increase is due to change in fee structure (especially from fee to free in some cases).

38-40 Increases are due to the inclusion of the UBCO campus.

BROWN

Medical library statistics cannot be disaggregated from the main because the medical collection is an integral part of the Sciences Library.

3 Basis of volume count is both physical and bibliographic since electronic books have been added.

4 Increase based on three factors: (1) purchase of new packages; (2) decision to outsource our e-journal holdings has required a very thorough review of e-journals, uncovering titles previously missed; (3) tools/data available from Serials Solutions have made us aware of titles that heretofore we were unaware.

18 Salary expenditures reflect change in reporting for JCB library. 2004-05 figure included both salaries and benefits.

\section{CALIFORNIA, BERKELEY}

Volumes held June 30, 2005 revised to 9,935,596.

4 Figure represents the best available estimate of both physical and electronic serials.

\section{CALIFORNIA, DAVIS}

Volumes held June 30, 2005 revised to 3,490,368 due to a recount of items on hand in the Agricultural Research Economics Library unit.

2 Increase due to a number of titles purchased to augment the Blaisdell Medical Library collection, in anticipation of medical curriculum moving to the Sacramento campus.

4a-b Increase due to the General Library tracking this item separately for the first time in 2005-06.

15 Overall costs for Library Materials for the Main Library in 2004-05 were \$5,247,950 due to prepayments made in 2003 04. Thus, the increase between years is not as large as it first appears.

15.a New and current academic program needs required an infusion of funds to cover monographs selected by bibliographers for purchase.

15.b Overall costs for Library Materials for the Main Library and Health Sciences Library in 2004-05 were understated due to prepayments of $\$ 1,042,553$ made in 2003-04.

15.d Increase due to a $23 \%$ increase in tax paid on monographs, in addition to memberships previously incorrectly reported in the monograph column.

20 Increase due to new and current academic program needs, an increase in payroll due to new academic and staff hires made during the reporting period, and systems enhancements.

23.a Actual costs are $\$ 108,762$, as OCLC was prepaid in the amount of $\$ 88,000$ for 2006-07.

\section{CALIFORNIA, IRVINE}

Volumes held June 30, 2005 revised to 2,494,986.

4 Increase due to increased collections and from corrections to 2004-05 data.

\section{CALIFORNIA, LOS ANGELES}

Volumes held June 30, 2005 revised to 8,088,635.

15.a $\$ 934,000$ of the increase in monograph expenditures reflects the acquisition of the Isadora Duncan collection in 200506.

23.b 2005-06 amount provided by University of California Digital Library. UCLA amount determined by prorating the total UC Centrally Financed Database Expenditures by 2005-06 actual enrollment by UC Campus.

24 Increase over 2004-05 due to increase in CLICC (College Library Instructional Computing Commons) computerrelated expenditures of $\$ 604,000$. 


\section{CALIFORNIA, SAN DIEGO}

Volumes held June 30, 2005 revised to 3,146,758 to correct a duplication count of a small set of volumes moved from a closing branch (CMRR) and absorbed into a larger branch (S\&E).

2 Total includes 32,030 e-books.

3 The print monographic volume count is physical. The e-book count is bibliographic.

12 2004-05 figure should have been reported as 116,046.

15.a Endowment expenditures were $\$ 350,000$ less due to late spring allocation.

15.b Includes monographs and publisher series, as well as all formats and electronic databases with annual subscriptions.

15.c Total is lower due to a $\$ 107,000$ ISI Web of Science purchase in 2004-05.

21 Decrease due to some materials previously included here, now included with Electronic Serials (22).

22 Includes some one-time expenses not included in Computer Files (21).

36 Includes 7 joint doctoral degrees with California State University at San Diego.

\section{CANADA INSTITUTE OF SCIENTIFIC AND TECHNICAL INFORMATION}

1.b.ii Figure reflects a weeding project whereby volumes were removed.

15-25 Expenditures as reported in Canadian dollars: (15a) \$541,506; (15b) \$11,399,733; (15c) \$1,489,499; (15d) $\$ 93,831 ;(15)$ $\$ 13,524,569 ;$; (16) UA/NA; (17a) UA/NA; (17b) UA/NA; (17c) UA/NA; (17) $\$ 18,987,177 ;$ (19) $\$ 13,965,431 ;(20)$ $\$ 46,477,177$; (21) \$1,514,073; (22) \$1,935,963; (23a) \$10,414; (23b) UA/NA; (24) \$2,636,236; (25) UA/NA.

27 Figure reflects the front desk only.

\section{CASE WESTERN RESERVE}

15.d Includes expenses for Dialog, CNI membership, ICPSR membership, ARL membership, SPARC membership, CLIR membership, TEI and theses charges.

\section{CHICAGO}

John Crerar Library is a central science library with a biomedical collection for health sciences. Many numbers are percentages as a result. Data for the ARL survey are matched as closely as possible to data compiled and reported for the Association of Academic Health Sciences Libraries' (AAHSL) Annual Statistics of Medical School Libraries in the United States and Canada.

Volumes held June 30, 2005 revised to 7,644,714 to include 281,165 electronic monographs, previously unreported.

2 Approximately $72 \%$ of this total represents a title count.

$8-10,12$,

Excludes unprocessed and partially processed items.

21 Increase represents an emphasis on backfiles and other one-time purchases, and a shift toward the purchase of packages rather than subscriptions when that option exists.

23.a Includes standard ongoing operating costs only.

23.b Figure is estimated.

35 Includes 433 requests filled by the Center for Research Libraries.

\section{CINCINNATI}

Volumes held June 30, 2005 revised to 3,125,908 to reflect 4,175 volumes added for Government Documents retrocataloging and 1,585 subtracted for Government Documents retro-cleanup.

3 Historical data are based on a bibliographic count. Additions and deletions are based on physical count.

5 Electronic government documents are counted, but print are not, unless cataloged in the LC collection.

7 Figure decreases each year due to government document retro-cataloging.

22 Increase due to the way consortial payments were made. There is flexibility to pay on either side of the fiscal year divide.

34 Increase due to OhioLINK - and within the community, Cincinnati's priority as lender changed from low to high. Figure includes the whole university, including the two two-year colleges - Raymond Walters College and Clermont 
College - which are typically excluded from these statistics.

35 Figure includes the whole university, including the two two-year colleges - Raymond Walters College and Clermont College - which are typically excluded from these statistics.

\section{COLORADO}

Volumes held June 30, 2005 revised to 3,578,229 to include 23,403 e-books that were added during the 2005-06 fiscal year.

1.b.ii Significant increase due to a large number of government documents withdrawals in 2005-06.

3 Count is about $90 \%$ bibliographic and $10 \%$ physical.

9 Decrease due to move of large collection from Archives to another university.

13 Decrease due to large number of these items in the Archives Department being transferred to another university.

15.c Large increase due to major one-time electronic purchases (e.g., ECCO).

15.d Includes: Non-contract binding $(\$ 8,296)$; GPO shelf list service $(\$ 4,745)$; III services $(\$ 106,981)$; interlibrary loan $(\$ 14,974)$, memberships $(\$ 212,752) ;$ OCLC utility $(\$ 132,913) ;$ projects $(\$ 86,190) ;$ RLIN utility $(\$ 1,348)$; storage facility in Boulder (\$27,540); Prospector $(\$ 4,225)$; reconciliation with Peoplesoft $(\$ 105)$.

21 Large increase due to major one-time electronic purchases (e.g., ECCO).

23.a Expenditures for consortia memberships and bibliographic utilities are always paid out of the materials budget (15d), not out of other operating expenditures (19).

25 Copy costs decreased due to purchase/use of scanner in ILL.

\section{COLORADO STATE}

1.b Decrease due to decrease in gross volumes added and increased withdrawals throughout the fiscal year.

1.b.i Decrease due to reduction in flood recovery funds.

4.b Figure reflects an increase in free e-journals.

6 Figure reflects a change in methodology for calculating - bibliographic record shows 8 units per volume.

10 Includes a correction for uncataloged maps.

12 Figure is a result of weeding.

15.c Additional backfiles were added.

26 Working with the University Strategic Plan, open positions were not immediately filled until the reevaluation was completed showing faculty and staff support skills needed to implement new strategies and programs.

33 Figure reflects fewer checkouts.

40, 42 Figures are a result of multiple strategies to increase student enrollment.

\section{COLUMBIA}

Volumes held June 30, 2005 revised to 9,299,036 to include 21,994 electronic government documents that were added in 2005-06.

2 Includes 30,522 e-books, representing the number of e-books added to the catalog in 2005-06. Some were purchased and not added to the catalog; those are not included here.

15.c Includes digitization of historical dissertation collections, art collections, and development of digital finance archive.

15.d Includes costs associated with maintenance of collection (e.g., shipping, supplies, relocation, memberships).

17.a,

17.c, 19, Includes data for The Columbia Center for New Media Teaching and Learning (CCNMTL), Digital Knowledge

24, 26.a, Ventures (DKV) and EPIC (Electronic Publishing Initiative at Columbia).

26.c

17.b, 26.b Includes data for The Columbia Center for New Media Teaching and Learning (CCNMTL).

20 Excludes fringe benefits of $\$ 569,472$. 
25 ILL for Barnard handled by Columbia University.

26.c Figure is an estimate.

36 Teachers College also awarded 153 Ed.D. degrees.

\section{CONNECTICUT}

Volumes held June 30, 2005 revised to 3,597,423 to include 327,747 e-books. Figures for volumes added (1.b) are for print monographs.

15.b Increased expenditure can be attributed to "spend-down" from Lost Book, Fines and Fees accounts; the dissolution of an "advance account"; and the transfer of new periodical subscription.

\section{CORNELL}

Volumes held June 30, 2005 revised to 7,666,969 to reflect additional e-book counts (Early English Books Online, 18th Century Collections Online, Evans Digital Edition, and NetLibrary), and a small print count correction.

1.b.i Includes a small number of e-books, from the Witchcraft Collection.

4 Includes some unnumbered monographic series. Reflects in part cancellations of print subscriptions for which electronic access continues, cancellations of duplicate print subscriptions, and other adjustments. Estimate of e-serials does not exclude non-current titles in packages with current titles.

9 Figure in cubic feet.

15.d Figure reflects shipping costs only.

21, 22 Excludes Health Science Library.

26 Does not include staff in positions that were temporarily vacant. Includes professional, support and student staff on grants and projects.

28 Part of one library remains open between 2 and 8 a.m., Sunday-Thursday, staffed by library-paid security staff, which adds 27.5 hours to this figure.

31 Some libraries' counts based on sampling.

32, 33 Includes ILL and some staff transactions.

40, 42 Employee degree only.

\section{CENTER FOR RESEARCH LIBRARIES}

1.b.i Not only does the number of hard copy deposits vary from year to year, but the number of institutions who send to CRL hard copy dissertations decreases as electronic dissertations increase.

17.b Vacant positions have been filled since 2004-05.

35 CRL occasionally borrows materials from other libraries in order to make a preservation microfilm copy of an item which is out of print. This happened 3 times in 2005-06.

\section{DARTMOUTH}

Volumes held June 30, 2005 revised to 2,648,633 to include one-time additions of e-books, including 94,543 e-books from Early English Books Online.

1.b.i For the first time, includes e-books: Early American imprints (36,307), Early Encounters in NA (146), IEEE Xplore $(3,582)$, John Wiley (22), Knovel (807), MARCIVE $(25,184)$, MIT Cognet (411), Netlibrary $(14,729)$, Psycbooks $(847)$, Women Writers Project (188), Miscellaneous $(1,962)$.

4.a Includes 35,680 e-journals.

4.b Includes 3,174 e-journals. Free e-journals are underreported, as there is no consistent way of identifying them. Figure reflects packages in Serials Solutions identified as free, plus MARCIVE serials.

5 Federal documents are included partially, namely those that were selected for local cataloging and classification. We purchased MARCIVE records, but did not add all document serials to our subscription count.

23.a We have decided to opt out of this question, feeling it is too poorly defined to provide a meaningful basis of comparison between libraries.

25 This year the accounting was revised to capture all ILL and document delivery transactions, which have been consistently underreported in the past. 
39 Includes 31 undergrad and 5 grad students displaced by Hurricane Katrina.

\section{DELAWARE}

9 Excludes the University Archives which is not administered by the Library.

26 Excludes professional and support staff in Information Technologies assigned to the Library.

\section{DUKE}

Volumes held June 30, 2005 revised to 5,569,053 to include 8,087 volumes omitted from 2004-05 data.

1.b, 1.b.i Increases apparent because 2004-05 included decreases due to data loss, a shortened fiscal year and the implementation of new workflows in a new ILS.

2 Increase due to an increased amount of monographs purchased.

4.a Increase due to the inclusion of full-text electronic journals; previously these data were not available.

8 Increase apparent because 26,501 items were omitted in 2004-05.

15.a Increase due to an increased collections budget.

15.c The main library is not able to disaggregate the expenses of various formats.

15.d Increase due to increased miscellaneous expenditures.

17.c Increase due to an increase in student assistants hired.

19 Increase due to an increase in operating expenditures, including printers and other peripherals purchased during library renovation.

21, 22 Increase due to an increase of expenditures in this area.

23.b The Business Library reported an increase in expenditures in this area.

27 Current Periodicals was consolidated with Microforms into one service point.

29, 30 Increase due to an increase in library presentations to groups.

34,35 Increase due to higher requests in this area.

39-42 Increase apparent because enrollment statistics now include both degree-seeking and non-degree-seeking students. Previously only degree-seeking students were reported.

\section{EMORY}

Volumes held June 30, 2005 revised to 3,107,528.

1.b.i Includes 894 e-books from: EBL (91) and Knovel (803).

19 2005-06 did not have extraordinary operating expenses as in 2004-05. 2004-05 figure included one-time costs for Dawson and renovations of level 1.

23.b GALILEO services and costs have expanded dramatically from 2004-05 to 2005-06.

39-42 In the 2004-05 ARL Statistics, fall 2005 enrollment data were inadvertently used rather than fall 2004 enrollment data. Thus, these figures show no change from 2004-05.

\section{FLORIDA}

4.b Increase from newly available count of electronic serials not purchased.

17.c Includes some hourly employees that may not have current student status.

29, 30 Humanities and social sciences library closed during 2005-06.

\section{FLORIDA STATE}

9 2004-05 figure excluded Pepper Library; 2005-06 figure is based on new measuring procedures and includes Pepper Library.

17 In 2004-05, fringe benefits were reported under Operating Expenditures (19); 2005-06 figure includes fringe benefits.

21 Includes 2005-06 databases and e-book collections, as there is no section for databases as for electronic serials.

32, 33 Includes reserves; new system cannot exclude them. 


\section{GEORGE WASHINGTON}

Volumes held June 30, 2005 revised to 2,167,580.

1.b The Main Library's count includes 252 e-books purchased (not subscribed to) and cataloged (via MARC tape load). 926 Dewey Decimal and reference items were withdrawn.

4 Number of full-text journals purchased has increased significantly since print and online journals count as print, but online-only journals count as online. Free journals include 2,868 e-journals linked via SFX plus 179 government document e-journals.

4.a The Main Library spent $\$ 268,303$ on one time electronic purchases: several large journal backfiles from Springer and Elsevier, as well as large one-time monographic type purchases which will be spread out over three years, like Early English Books Online and North American Immigrant Letters, Diaries and Oral Histories. This trend of one-time electronic purchases is expected to continue and possibly increase.

15.b The law school had some year-end funds, and some 2006-07 subscriptions were paid for at the end of 2004-05 instead of holding them until 2005-06. Also, inflation was a factor.

15.c In 2004-05 the Law Library purchased some large microform sets; decrease is due to those purchases not being needed in 2005-06.

19 Increase in 2005-06 is due mainly to the cost of numerous construction and renovation projects.

20 The fee-based Gelman Library Information Service ceased operations on December 31, 2005.

25 Starting in 2005-06, the Main Library began including the costs for Science Direct Doc Delivery.

\section{GEORGETOWN}

2,15.a Increases due to the addition of a branch library.

\section{GEORGIA}

Volumes held June 30, 2005 revised to 4,274,074 to include EBBO volume count added, which was not included in 2004-05.

21 Decrease due to Law Library having fewer one-time purchases.

23.a Decrease due to Law Library having one-time bibliographic purchases in 2004-05.

25 Decrease due to fewer expenses in 2005-06.

\section{GEORGIA TECH}

25, 35 Increased volume due to greater ILL subsidy.

\section{GUELPH}

1.a Collections of electronic books that the Library "owns" but has yet to catalogue and make ready for use are not included in this figure. One example would be Eighteenth Century Collections Online.

1.b The percentage change from 2004-05 is large but the absolute change is not significant.

4.b Figure reflects the number of free electronic journals available through the Library's Web site.

15-25 Expenditures as reported in Canadian dollars: $(15 a) \$ 1,214,493 ;(15 b) \$ 3,773,958 ;(15 c) \$ 369,874 ;(15 d) \$ 501,543 ;(15)$ $\$ 5,859,868$; (16) \$32,153; (17a) \$2,770,228; (17b) \$2,997,448; (17c) \$290,926; (17) \$6,058,602; (19) \$1,322,166; (20) \$13,272,789; (21) \$292,322; (22) \$3,427,390; (23a) \$152,298; (23b) \$0; (24) \$562,771; (25) \$216,104.

19 The decrease in this figure relative to 2004-05 reflects the fact that in 2005-06 the University did not offer a "Voluntary early retirement and resignation" program.

23.a Marked increase relative to 2004-05 is due to substantial outsourcing of book cataloguing.

25 Increase in figure relative to 2004-05 reflects dramatic growth in the purchase of digital rights to scholarly documents as part of the Library's "e-learning" operations. The "e-learning" expenditures could not be separated from traditional DD/ILL expenditures.

29 The increase in number of presentations (relative to 2004-05) is a reflection of the importance given to promoting information literacy, as well as better record keeping.

36 Number of Ph.D. graduates is for the period October 2005 to September 2006. 
Volumes held June 30, 2005 revised to 15,586,234.

1 Figure reflects changes in acquisition patterns, including growth in electronic resources.

15 Decrease in (15d) reflects a change in accounting practice for electronic resources in faculty library. Increase in (15c) largely reflects acquisition of electronic resources.

\section{HAWAII}

Volumes held June 30, 2005 revised to 3,482,705 to reflect gift volumes inadvertently omitted by the Law Library in 2003-04.

1.b.ii Includes materials lost in the October 2004 flood.

2 Reflects increased ordering capability following October 2004 flood.

4.a Reflects a combination of serial cancellations and a new count of serial orders.

4.b Includes electronic serials not previously reported.

6 New physical count of microforms following October 2004 flood.

10 Reflects cartographic materials lost in the October 2004 flood.

15.a Reflects increased approval plan receipts following interruption of acquisitions activities due to the October 2004 flood. Includes audiovisual media not previously included.

15.c Figures for previous years included audiovisual media which are now included with monographs.

16 Figure reflects budget shortfall.

17.a-b Figures reported in previous years were estimates.

23.a Includes ARL, CNI, GWLA, OCLC, RLG.

24 Includes maintenance fees not previously reported.

25 May reflect increased librarian-student interaction following a period of curtailed library instruction activity due to the October 2004 flood.

29, 30 Increase from 2004-05 reflects impact of October 2004 flood on library instruction activity.

31 Includes simple directional questions.

34 2004-05 figure revised to 4,406.

35 2004-05 figure revised to 11,038 . Revised $60 \%$ increase may reflect increased librarian-student interaction following a period of curtailed library instruction activity due to the October 2004 flood.

\section{HOUSTON}

Volumes held June 30, 2005 revised to 2,160,366 to reflect a revision by the Law Library.

2 Both UH Law Library and the Main Library had a significant increase in number of monographic volumes purchased.

6 Increase due to a change in the way the Law Library reported its statistics. Previously, they had used the definition required for the American Bar Association (fiche = number of units divided by 6, microfilm = number of reels $\times 5$ ). 2005-06 figure reflects the definition used by ARL and was calculated by reverse-engineering the figures reported to ABA.

8, 9 Excludes Law Library.

22 UH Libraries report a significant growth in expenditures in this area. These expenditures are matched by the growth in number of electronic journals purchased in the ARL Supplementary Statistics.

26.a Five positions that were reported as professional in 2004-05 are reported as support staff in 2005-06.

\section{HOWARD}

Volumes held June 30, 2005 revised to 2,401,460.

17.c In 2005-06 students used all available hours; in 2004-05 they did not.

19 MSRC did not report any figure in 2004-05.

22 Excludes Health Sciences Library. 
24 No figure reported for Main Library in 2005-06.

31 MSRC did not report any figure in 2005-06.

32 More reliable record-keeping system used in 2005-06.

33 Figure reflects renewals; more reliable record-keeping system used in 2005-06.

34, 35 Increased access to full-text databases.

\section{ILLINOIS, CHICAGO}

1.b Excludes electronic titles (according to records, no permanently owned volumes were added/withdrawn), PromptCat titles (shelf-ready), government documents processed by that department, and Special Collections cataloging not performed through the catalog department.

12 Excludes videocassettes.

13 Does not include $16 \mathrm{~mm}$ films (non-circulating).

15.d Figure reflects expenditures for standing orders.

17 Includes both Richard J. Daley Library and LHS. Separate figures unavailable.

$21,23 a-$
b, 25 Figures cannot be separated from (15).

24 Figure cannot be separated from data in (15) and (19).

35 Figures unavailable for all institutions with whom UIC has reciprocal borrowing privileges.

\section{ILLINOIS, URBANA}

Volumes held June 30, 2005 revised to 10,371,460.

4.a Change in this figure results from change in reporting method from physical to bibliographic data pulled from the ILS system.

16 Decrease results from transfer of expenditures to contract conservation budget.

\section{INDIANA}

21 2004-05 figure included large purchases of periodical backfiles and literature collections.

31.a New Reference Portal tracks actual reference transactions.

33 Improved data retrieval detected double-counting of some areas in previous years.

\section{IOWA}

1.b.ii Government publications coming in electronic format supercede older versions of print. The older print volumes are then withdrawn, causing an increase in this figure.

21 Figure includes a grant from a private foundation for just under $\$ 300,000$ to purchase serial backfiles.

22 Increase due to purchase of electronic resources only rather than both electronic and print.

27 Due to several reorganizations, a number of service points have been combined or eliminated. Correct data should have been provided several years ago but due to staff responsibilities they were not.

31 Figure is affected by the week that is counted during the semester; sampling occurs 1 week each semester or 3 times per year.

32 Figure available for Law Library, but unavailable for Main Library.

38 Does not include 533 clinical and pre-clinical faulty with departmental appointments.

\section{IOWA STATE}

15.c Increase is due to increased purchases of backsets of electronic resources and the recording of certain expenditures in (15c) rather than (15a).

17a, 19 Salaries increased \$150,874, leaving less money for Other Operating Expenditures, causing the changes in these figures.

21 Increase is due to increased purchases of electronic resource backsets.

22 Library increased its Electronic Serials purchases by \$2,094,244 in 2005-06. 
24 Decrease reflects a management plan that includes saving money by buying PCs at bulk rates and working out a new three-year rotating schedule to replace PCs.

27 Closed the Periodical Room Service Desk July 2005.

\section{JOHNS HOPKINS}

Volumes held June 30, 2005 revised to 3,641,641 to reflect a more accurate count.

KANSAS

17.a Increases due to a $27^{\text {th }}$ pay period; due to bi-weekly pay periods, this anomaly occurs once every six years.

\section{KENT STATE}

Continued staffing changes have resulted in reporting anomalies over the past several years.

\section{KENTUCKY}

Volumes held June 30, 2005 revised to 3,325,894 to include 39,163 e-books purchased in 2005-06 (Early American Imprints: 38,095; Evan's Supplement: 1,068)

17, 20, 26 Some Law Library support staff FTE included in Main Library total in 2004-05. 2004-05 figures for Main Library revised to: (17.a) \$3,266,525; (17.b) \$2,061,825; (17) \$6,026,458; (20) \$14,859,781; (26.a) 69.5; (26.b) 93; (26) 217.

\section{LAVAL}

1.b.ii This figure represents a huge decrease over 2004-05, when a lot of weeding was done in the Science Library.

4.b 2004-05 figure was inaccurate; some free titles were counted by mistake. While accessible, they were not in the catalog.

10 Includes 27,497 geographic files.

15-25 Expenditures as reported in Canadian dollars: $(15 a) \$ 2,500,058 ;(15 b) \$ 7,429,780 ;(15 c) \$ 104,821 ;(15 d) \$ 200,500 ;(15)$ $\$ 10,235,159 ;$; (16) \$54,510; (17a) \$3,691,209; (17b) \$5,656,870; (17c) \$451,437; (17) \$9,799,516; (19) \$488,397; (20) $\$ 20,577,582 ;$ (21) \$584,307; (22) \$4,443,530; (23a) \$202,923; (23b) \$0; (24) \$426,037; (25) \$110,948.

17 Decrease in salaries is explainable by the fact that there are many new librarians.

17.c Many assistants were hired for digitization projects.

21 Includes JSTOR, electronic geographic material, and Journals Backfiles collections.

24 Includes: Service contracts for hardware $(\$ 32,065)$; computer hardware $(\$ 359,838)$; and software $(\$ 34,133)$.

33 A courtesy e-mail system was implemented, advising users that the loan is going to expire in a few days. That may be the cause for the change in this figure from 2004-05.

\section{LIBRARY AND ARCHIVES CANADA}

All figures are as of March 31, 2006.

Volumes held June 30, 2005 revised to 8,697,894.

15-25 Expenditures as reported in Canadian dollars: (15a) \$854,779; (15b) \$1,025,429; (15c) UA/NA; (15d) UA/NA; (15) $\$ 1,880,208$; (16) \$7,895; (17a) UA/NA; (17b) UA/NA; (17c) UA/NA; (17) UA/NA; (19) UA/NA; (20) $\$ 1,888,103 ;(21)$ UA/NA; (22) UA/NA; (23a) UA/NA; (23b) UA/NA; (24) UA/NA; (25) UA/NA.

17, 20 Due to staffing changes related to reorganization, these expenditures are reported as UA/NA.

\section{LIBRARY OF CONGRESS}

All figures are as of September 30, 2006.

1.a All figures for the fiscal year October 1, 2005 through September 30, 2006.

4 Figure reflects an approximate count. Reports for previous years counted number of titles, not subscriptions.

17.a Includes wages of support staff and student assistants.

\section{LOUISIANA STATE}

Volumes held June 30, 2005 revised to 3,468,236 to include: Netlibrary (2,589); Early English Books Online (94,758); Black Thought \& Culture (175); North American Women Letters and Diaries (463); Women's Social Movement (122); Early American Fiction Full Text Database (882). 
1.b.ii An additional branch library was closed and an extensive weeding project was undertaken to eliminate duplicates and books that were not circulating or were outdated.

2 Decrease from 2004-05 is due to one-time additions of large e-book packages in 2004-05.

10 Change due to the report run in 2005-06 giving different data than in 2004-05.

21 Change due to a revised counting method.

\section{LOUISVILLE}

Volumes held June 30, 2005 revised to 2,016,208.

\section{MCGILL}

4 2004-05 figure revised to 74,972 to reflect the inclusion of electronic serials as per ARL instructions.

15-25 Expenditures as reported in Canadian dollars: (15a) \$4,008,693; (15b) \$7,790,403; (15c) $\$ 1,207,410 ;(15 d) \$ 424,819 ;(15)$

$\$ 13,431,325$; (16) $\$ 229,982$; (17a) $\$ 5,544,270$; (17b) $\$ 5,946,028 ;(17 \mathrm{c}) \$ 721,866 ;(17) \$ 12,212,164 ;(19) \$ 4,225,211 ;(20)$

$\$ 30,098,682$; (21) \$714,768; (22) \$6,282,163; (23a) \$216,801; (23b) UA/NA; (24) \$809,765; (25) \$168,710.

\section{MCMASTER}

15-25 Expenditures as reported in Canadian dollars: (15a) $\$ 1,563,786 ;(15 b) \$ 5,629,371 ;(15 c) \$ 490,593 ;(15 d) \$ 257,958 ;(15)$

\$7,941,708; (16) \$145,753; (17a) \$2,157,960; (17b) \$3,986,980; (17c) \$403,625; (17) \$6,548,565; (19) \$1,209,630; (20)

$\$ 15,845,656$; (21) \$738,739; (22) \$4,111,122; (23a) \$124,493; (23b) \$0; (24) \$366,372; (25) \$109,939.

\section{MANITOBA}

All figures are as of March 31, 2006 and include: William R. Newman Agriculture Library; Architecture/Fine Arts/Music Libraries; Archives \& Special Collections; Elizabeth Dafoe Library; Fr. Harold Drake Library; St. John's College Library; D.S.Woods Education Library; Donald W. Craik Engineering Library; E.K. Williams Law Library; Albert D. Cohen Management Library; Sciences and Technology Library; Neil John Maclean Health Sciences Library; Bill Larson Library; Carolyn Sifton-Helene Fuld Library; Concordia Hospital Library; J.W. Crane Memorial Library; Misericordia Health Centre Library; Riverview Health Centre Virtual Library; Seven Oaks General Hospital Library; Victoria General Hospital Library.

15-25 Expenditures as reported in Canadian dollars: (15a) $\$ 2,119,234 ;(15 b) \$ 5,265,734 ;(15 c) \$ 110,463 ;(15 d) \$ 364,505 ;(15)$ $\$ 7,859,936$; (16) $\$ 153,562 ;$ (17a) \$4,851,363; (17b) \$5,282,742; (17c) \$800,386; (17) \$10,934,491; (19) \$3,419,734; (20) \$22,367,723; (21) \$556,069; (22) \$3,108,206; (23a) UA/NA; (23b) UA/NA; (24) \$404,121; (25) \$191,127.

\section{MARYLAND}

Volumes held June 30, 2005 revised to 3,465,506 to include government documents not previously included in volume counts.

25 Libraries began using ILLIAD in 2005-06.

\section{MASSACHUSETTS}

1.b.ii Withdrawals are abnormally high due to weeding of added copies from a traditional print-oriented reserve reading collection.

7 The accuracy of this number has eroded over the past few years and is no longer reliable. There is a large group of extant items in this category, but its magnitude is in question.

16 Continuing conversion of print journal subscriptions to access in digital format has decreased the need for contract binding.

17.c Includes federal work-study funds/subsidy.

24 Cyclical renewal of technology assets and expansion of the Learning Commons resulted in a substantial increase in expenditures for hardware and software.

27 Implementation of the Learning Commons resulted in a net increase of 3 staffed service points.

28 Implementation of the Learning Commons with its $24 / 5$ service schedule resulted in a large increase in service hours.

32 Circulation increased coincident with implementation of the Learning Commons.

34 Includes the numbers of items provided to two separate groups that also participate in Virtual Catalog projects; items paged from the stacks and sent to partner libraries in the Five College Library consortium; and traditional ILL, RAPID, and Document Delivery activities.

36 Excludes 30 Ed.D. degrees. No doctoral degrees were awarded for Audiology (Au.D.) or for Nursing Practice (DNP). 
37 Excludes Ed.D. (Education), Au.D. (Audiology), and DNP (Nursing Practice) doctoral degree programs.

MIT

19 2004-05 figure revised to $\$ 1,944,151$.

MIAMI

Volumes held June 30, 2005 revised to 2,952,532 to include 359,676 e-books not included in prior years. E-book collections include NetLibrary, Early English Books Online, Gale's 18th Century Collections Online, Women Writers Project, Wright American Fiction, Making of the Modern World, and Making of Modern Law.

17.a Excludes salary of the University Librarian. Includes salaries of 40 librarians and 3 non-librarian administrative/professional employees in central library; 10 exempt employees in central library reported as professional staff in 2004-05 are reported as support staff in 2005-06.

17.b In central library 10 exempt staff reported as professional staff in 2004-05 are reported as support staff in 2005-06.

17.c Excludes wages for student assistants subsidized by the School of Business Administration.

26.a Includes 40 librarians and 3 non-librarian administrative/professional employees in central library; 10 exempt employees in central library reported as professional staff in 2004-05 are reported as support staff in 2005-06.

26.b In central library 10 exempt staff reported as professional staff in 2004-05 are reported as support staff in 2005-06, for a total of 90 support staff in central library.

31 Reference transactions for marine and architecture libraries are unavailable. Reference transactions are based on enumerations in central, business, and music libraries and sampling in law and medical libraries.

37 Number of Ph.D. fields was recalculated in 2005 by University administration; reported number of Ph.D. fields in 2005 is a result of renaming programs and recombining majors and not a decrease in fields.

\section{MICHIGAN}

Volumes held June 30, 2005 revised to 8,138,604.

\section{MICHIGAN STATE}

1.a Includes 17,926 NetLibrary plus 34,542 Gov Doc e-books.

1.b.i Includes 7,777 NetLibrary plus 4,059 Gov Doc e-books.

4.a Includes monographic series that cannot be disaggregated.

15.b Includes expenditures for monographic series that cannot be disaggregated.

15.c Main library expenditures are included in monograph expenditures (15a) and current serials expenditures (15b). Figure reflects Law Library only.

15.d Includes: ILL Reserve (\$91,164); CRL Reserve $(\$ 44,763)$.

22 Increase reflects continuing conversion of large numbers of journals from print-based pricing, to a model where the online version is the basis of the subscription.

23.b MSU Libraries have access to a large number of electronic resources from several vendors through the Michigan eLibrary. These resources are provided to all residents of the State of Michigan.

29, 30 Includes tours and orientations for the first time.

39-42 2004-05 figures revised to: (39) 38,557; (40) 6,279; (41) 6,859; (42) 2,569.

\section{MINNESOTA}

Volumes held June 30, 2005 revised to 6,610,156 for adjustments to unit collection counts, corrections to records, and approximately 25,000 government documents records loaded.

1.b.ii Increase in withdrawals is largely due to an extensive duplicate withdrawal project in conjunction with moving materials to on-site storage.

2 Count reflects monograph titles ordered, not volumes purchased. Increase is due to $\$ 500,000$ increase in allocations for monograph purchases.

4 Increase in serials count is due to full count of individual titles in aggregated e-serial packages. These were not captured in counts submitted in past years.

4.a Increase is due to full count of individual titles in aggregated e-serial packages. These were not captured in counts 
submitted in past years.

4.b Includes 16,000 electronic titles provided by ELM consortium (Electronic Library for Minnesota) that were not captured in counts submitted in previous years.

11-13 Breakdown for Audiovisual materials not available. Aggregated A/V materials count for ARL Main Library is 859,171 .

Aggregated A/V materials count for ARL statistics including Health Sciences and Law is 862,627.

15.a Increase is due to $\$ 500,000$ increase in allocations for monograph purchases.

15.c Increase includes significant purchases of electronic serials backfiles. Also, in 2004-05 not all serial backfile purchases were included.

17.a Increase due to increases to professional salaries based on market equity study.

21 Increase reflects significant expenditures on electronic and print serial backfiles.

25 Includes expenses for Interlibrary loan borrowing and lending only. Does not include fee-for-service document delivery service for external users. This was included in 2004-05 and accounts for the apparent decrease. Expenses for regular ILL services increased slightly.

30.a Some participant numbers reported by some units are estimated.

31 Reference transactions are mainly counts; a few units' figures are estimated.

33 Increase in renewals reflects is due to increased use of online renewal facility.

36 Total includes all doctoral degrees awarded for 2005-06. Includes Health Sciences and Law academic units.

37 Includes Health Sciences and Law academic units.

38 Full-time primarily instruction non-medical faculty reported in EAP survey. Includes Health Sciences and Law academic units.

39-42 Includes Health Sciences and Law academic units. Graduate student count includes all post baccalaureate students (graduate/professional).

\section{MISSOURI}

4a, 4b Figures based upon best calculations from Acquisitions, LSO, HK counts, and the Open URL resolver.

15.b Includes $\$ 773,414$ serials paid by system-wide Library Systems Office.

15.c Includes memberships.

17 Excludes $\$ 1,459,912$ of fringe benefits now paid by the MU Libraries on all full-time salaries.

17.a Includes $\$ 198,152$ from system-wide Library Systems Office.

19 Includes: Library Systems Office, including from gift funds and lapsed salaries $(\$ 143,546)$; capital building improvements (\$650,651, including $\$ 250,000$ on a new Information Commons); non-salary development expenditures $(\$ 90,165)$.

22 Includes $\$ 773,414$ from Library Systems Office.

26.a Includes 4 FTE from Library Systems Office and 3 FTE non-librarian professionals: Head of IT Services, Development officer, and Personnel coordinator.

26.c Figure computed from dividing total student wages (17c) by average pay of $\$ 6.50 /$ hour.

\section{MONTREAL}

Volumes held June 30, 2005 revised to 3,044,277 in order to correct past miscalculations.

4.a Increase due to an accelerated development of electronic resources.

15-25 Expenditures as reported in Canadian dollars: (15a) \$2,280,412; (15b) \$9,447,540; (15c) \$116,604; (15d) \$204,484; (15) $\$ 12,049,040$; (16) \$250,400; (17a) \$7,179,974; (17b) \$11,841,388; (17c) \$100,500; (17) \$19,121,862; (19) \$1,475,591; (20) \$32,896,893; (21) \$442,325; (22) \$5,407,195; (23a) \$188,140; (23b) \$0; (24) \$560,483; (25) \$174,253.

\section{NATIONAL AGRICULTURAL LIBRARY}

All figures are as of September 30, 2006. Volumes held June 30, 2005 revised to 2,396,472. 
25 Document delivery expenditures decreased due to a significant reduction in the number of document delivery requests received. This was, in turn, due to the increasing availability of full-text articles through NAL's DigiTop service, and the implementation of an end-user fee-for-service plan.

29 Includes exhibits.

32 Loan requests decreased due to (1) increasing availability of digital full-text resources and (2) implementation of an end-user fee-for-service plan.

35 Materials received from other libraries decreased sharply due to increasing availability of digital full-text resources and implementation of an end-user fee-for-service plan.

\section{NATIONAL LIBRARY OF MEDICINE}

All figures are as of September 30, 2006.

\section{NEBRASKA}

Volumes held June 30, 2005 revised to 3,079,188 to reflect ECCO titles added during 2005-06.

\section{NEW MEXICO}

1.b.ii Estimate 30,000 volumes "withdrawn" due to loss in a fire on April 30, 2006. Actual figure not yet determined.

4 Increase due to ILS re-coding project which provided data that was previously irretrievable.

15.c Number of backfiles and other materials vary greatly from year to year.

25 Use of DDS and ILL expanded due to fire.

32 Circulations down due to temporary closure and limited services after initial opening of library after fire.

\section{NEW YORK STATE LIBRARY}

All figures are as of March 31, 2006.

\section{NEW YORK}

Volumes held June 30, 2005 revised to 5,035,331 to include 769,197 e-books, up from the 2004-2005 count of 673,590 due to collections acquired in 2005-06.

2 Increase caused by catch-up of backlogged materials (books previously awaiting selector review have now been processed and added to the collection). Also, part of large binding credit with vendor was applied to current year allowing additional materials to be purchased.

4.b Increase due to a growing number of new sources offering open-access publishing and existing sources expanding their offerings.

11 Brought in large graphics collections to the Tamiment Labor Archives Library.

13 Aggressive conversion of video to DVD.

15.a Increase caused by three factors: 1) Catch-up of backlogged monographs (books previously awaiting selector review have now been added); 2) Large binding credit with vendor allowed additional materials to be purchased; 3) 20042005 stats were unusually low, thus inflating the variance between the 04-05 and 05-06 years.

15.b Part of the 2004-05 binding credit was applied here, allowing additional materials to be purchased.

15.c Figure reflects far less spent on e-journal backfiles.

16 A large proportion of 2005-06 actual binding services were prepaid in 2004-05.

19 Unusual personnel vacancies within 2005-06 allowed spending for other uses, such as furniture and equipment.

21 Figure reflects far less spent on e-journal backfiles.

22 Figure reflects rapid growth in movement from print to electronic serials.

24 Figure reflects fewer funds allocated for year-end computer equipment purchases.

29 An increased need for instructional programs resulted in more presentations given.

34 New RAPID membership has allowed for improved lending efficiency due to more accurate requests and a mandatory 24-hour turnaround time.

35 Increase due to RAPID membership, new borrowing privileges for undergraduates, improved discovery methods, and new academic programs and fields of research. 


\section{NORTH CAROLINA}

1.a, 2 Includes government documents.

15.d Includes payments for CRL membership.

20 Includes $\$ 4,102,193$ in grants, trust, and other special funds (without benefits); excludes $\$ 304,109$ in expenditures for the Triangle Research Libraries Network from funds contributed by Duke, North Carolina State, and North Carolina Central Universities.

26 Includes 4.35 professionals on special grant funds; 0 FTE on special projects. Excludes 2.25 FTE paid from funds contributed by Duke, North Carolina State, and North Carolina Central universities for support of the Triangle Research Libraries Network.

37 Figure taken from new source in 2005-06 to reflect strictly Ph.D. programs, resulting in lower figure than in 2004-05.

38 Decrease due to recalculation of data from new source more consistent with ARL definitions. Previous figures did not exclude all categories set forth in ARL definition.

\section{NORTH CAROLINA STATE}

2 Includes some monographic standing order items paid from serials funds. Includes 2,985 e-books.

15.a-b Some monographic titles are paid from serials funds included in (15b).

15.d Includes bibliographic utilities $(\$ 177,285)$; document delivery $(\$ 133,600)$; and memberships $(\$ 218,544)$.

19 Increases for 2005-06 are due in part to temporary relocations of personnel during construction and purchases related to a new learning commons.

\section{NORTHWESTERN}

1-2 Includes government documents.

8, 13 Excludes Health Sciences Library.

11 Excludes Health Sciences and Law Library.

21 Computer file expenditures redefined internally.

$31 \quad$ Health Sciences revised definition of reference transaction.

$412004-05$ figure revised to 6,675.

\section{NOTRE DAME}

2 The number of e-books has not been determined.

9 This figure represents the entire holdings. It consists primarily of manuscripts and archival records, but it also includes substantial quantities of printed, microfilm, audio, visual, film, graphic, digital, and artifactual materials as well. Controls over records are based primarily on the creator of the records, and only secondarily on the types of materials. University Archives was not included in prior years.

15.d Consists primarily of memberships for the purpose of obtaining/accessing publications and literature searching.

17a, 26a Figures reflect exempt employees.

17b, 26b Figures reflect non-exempt employees.

38 Increase due to reclassification of people based on a reevaluation of faculty positions for classification in a newly implemented HR system and for compliance with IPEDS definitions. The greatest number of faculty were reclassified from non-instructional special research/professional positions to instructional special research/professional positions. These people were deemed to have met the teaching threshold established by IPEDS.

OHIO

Volumes held June 30, 2006 revised to 2,654,701 to include a one-time download of catalog records associated with the purchase of Early American Imprints, Series I (Evans).

2 Large increase in number of volumes purchased is due to purchases of major e-book collections and an extensive collection of Southeast Asian materials.

4.a Increase due to error in counting paid subscriptions in 2004-05; subscriptions bundled in packages were inadvertently counted as single subscriptions. 
15.d Includes: Bibliographic utilities $(\$ 74,936)$; document delivery $(\$ 3,211)$; and memberships $(\$ 51,315)$.

21 2004-05 figures revised to: (21) \$33,426; (22) \$2,098,551.

\section{OHIO STATE}

Volumes held June 30, 2005 revised to 6,117,141 to agree with the item record count in the online system, and to include 136,436 ECCO titles paid for over a 3 year period that were added to the catalog in 2005-06. Volume counts for libraries added this year are 37,434 for the additional campus libraries and 279,000 for the branch campus libraries.

2 Includes 732 microfilm and 4,211 microfiche.

7 Cataloging has been completed for all printed U.S. Government Documents; only State of Ohio documents remain uncataloged.

12 Audio, film, and video are included in a single category in the OSU catalog. 2004-05 figure should be revised to 58,989 .

27 The Thompson (Main) Library had a number of separate service points that have been eliminated and will not be included in the renovated library.

31 Switched from manual to online data gathering, and the results did not accurately reflect the reference activity, so the figure has been made unavailable.

32 Includes libraries as identified above (totals for branch campuses: (32) 35,011; (33) 102,770).

34 Includes OhioLINK direct loans: (34) 46,098; (35) 70,997.

\section{OKLAHOMA}

Volumes held June 30, 2005 revised to 4,855,285 to reflect the inclusion of Schusterman Librqary.

1.b.i Increased acquisition of e-books as incorporated into collection development policies.

1.b.ii Conducted a de-selection project.

4 Increased acquisitions of electronic serials.

7 Increased acquisitions of depository items.

15.d Figure reflects changes in internal accounting practices.

17.b Figure reflects a change in method of reporting Professional Staff.

21 Increased desiderata purchases.

23.a 2004-05 figure did not include payments from deposit accounts. Deposit accounts no longer utilized.

29 Law Librarians began teaching a required, semester-long 1 credit hour course on legal research to the entire first-year law student body.

34 Began participating in Rapid ILL.

\section{OREGON}

Volumes held June 30, 2005 revised to 2,756,575 to include 98,046 records loaded for ECCO.

2 Excluded 98,046 titles in ECCO.

9 Figure is in linear feet.

10 Corrected from 2004-05, based on new inventory.

15 Excludes $\$ 325,000$ delayed payment.

15.a Includes \$52,950 for first of 4 payments for ECCO.

15.b \$325,000 payment was delayed and not included here, which will skew numbers for both 2005-06 and 2006-07.

15.c Included in (15a) and (15b).

21 Figure reflects ECCO (1st of 4 payments) and JSTOR III.

22 Does not include \$325,000 payment delayed until 2006-07. This will skew numbers for both 2005-06 and 2006-07.

24 Excludes library expenses to support campus Blackboard system and campus classroom equipment expenses. 
25 Includes subsidized pay-per-view. Law ILL expenses cannot be separated out.

26.a Figure now reflects the same list of professional staff as on the salary survey.

26.b Now use same split of OAs into professional and support staff as used in the ARL Annual Salary Survey.

\section{PENNSYLVANIA}

Volumes held June 30, 2005 revised to 5,784,556.

\section{PENNSYLVANIA STATE}

15b, 15c Fluctuation due to 2005-06 expenses being categorized differently than in prior years. Several acquisition types previously reported in (15c) are now being included in (15b).

33 The fluctuation from 2004-05 is due to the use of a new reporting tool, which allowed for increased accuracy.

\section{PITTSBURGH}

Volumes held June 30, 2005 revised to 4,786,298.

29-31 2005-06 statistics were tracked online.

\section{PRINCETON}

4 Decrease due to the serials budget remaining constant, while prices of the serials increased.

21, 22 In the past e-journals and electronic non-continuation acquisitions were not tagged, to distinguish between them for statistical purposes, and the counts reported were guesses at the breakdown. Tagging procedures have since been amended to provide actual counts for each category.

\section{PURDUE}

1-15 Includes cataloged government documents.

2 Represents number of titles rather than volumes. Excludes materials purchased on sponsored research programs.

4a-b Excludes some numbered monographic series.

4.b Includes cataloged depository, exchange, and gift serials, and electronic journals purchased through statewide consortium.

15.c Represents bibliographic and full-text databases for which expenditures can be tracked separately. Expenditures for other database and for materials such as maps and audiovisuals are included in those for monographs (15a) and serials (15b), and cannot be disaggregated.

22 Includes expenditures for bibliographic and full-text databases in (15c), plus electronic journals.

25 Includes $\$ 154,145$ of expenditures recovered by fee-based service.

29, 30 Excludes use of online, interactive tutorial, "CORE" (approximately 1,440 account holders plus unregistered users made 12,206 visits).

31 Includes 3,151 reference transactions via digital reference service.

34 2004-05 figure revised to 23,903 to correct errors in data reported by ILL management software.

\section{QUEEN'S UNIVERSITY}

All statistics unless otherwise stated are as at April 30, 2006.

15-25 Expenditures as reported in Canadian dollars: (15a) $\$ 1,406,799 ;(15 b) \$ 7,110,204 ;(15 c) \$ 134,045 ;(15 d) \$ 514,411 ;(15)$

$\$ 9,165,459 ;$; (16) \$100,335; (17a) \$2,668,876; (17b) \$4,460,434; (17c) \$294,483; (17) \$7,423,793; (19) \$2,217,879; (20)

$\$ 18,907,466$; (21) UA/NA; (22) \$5,207,820; (23a) \$180,303; (23b) \$174,479; (24) \$103,020; (25) \$56,610.

26.a-b Figures are as of November 2005.

36-37, Figures are as of November 2005.

38 Figure is as of October 2005.

RICE

1.a Includes 23,476 Texshare e-books, 36,434 government document e-books, and 749 non-document e-books.

1.b.i Includes 3,515 government document e-books and 119 non-document e-books. 
9 Manuscripts and archives reported in cubic feet.

15.d Includes memberships (\$76,462), ILL $(\$ 28,883)$, in-house binding supplies (\$26.355), and bibliographic utilities $(\$ 191,374)$.

\section{ROCHESTER}

3 Basis of count for 2,425 volumes is physical.

9 Count for the Sibley Library unavailable.

18 Fringe benefits not included for Sibley Library salaries and wages.

22 Does not include print plus online subscriptions.

24 Sibley Library figure is unavailable.

29.a Sibley Library presentations, 159 based on sampling.

30.a Sibley Library data based on sampling.

32,33 Includes reserves.

36, 37 Figure reflects Ph.D.s only for entire university. Past counts may have included other doctoral degrees.

\section{RUTGERS}

Volumes held June 30, 2005 revised to 4,164,903 to reflect 4,444 additional volumes withdrawn in 2005-06.

1.b.ii Performed special withdrawal project to remove items from catalog that have been missing from 1997-2004.

10-13 Audiovisual Materials figures cannot be disaggregated. Total audiovisual is 147,362.

23.a Changed method of calculation to more accurately reflect actual annual cost.

\section{SASKATCHEWAN}

15-25 Expenditures as reported in Canadian dollars: (15a) \$2,549,830; (15b) \$6,288,869; (15c) UA/NA; $(15 \mathrm{~d}) \$ 189,781 ;(15)$ $\$ 9,028,480$; (16) $\$ 84,069$; (17a) $\$ 3,348,349$; (17b) $\$ 3,179,954$; (17c) $\$ 308,295 ;(17) \$ 6,836,598 ;(19) \$ 1,296,175 ;(20)$ $\$ 17,245,322$; (21) \$19,435; (22) \$4,566,908; (23a) \$68,156; (23b) \$0; (24) \$585,802; (25) \$72,534.

25 2004-05 statistics were unusually high due to late payment.

\section{SMITHSONIAN INSTITUTION}

All figures are as of September 30, 2006.

\section{SOUTHERN CALIFORNIA}

4 Increase due to a combination of increased subscriptions (via increased funding and targeting new serials subscriptions as a result of user feedback), and more accurate counting with Serials Solution EMS.

\section{SOUTHERN ILLINOIS}

1.b Change reflects 2004-05 bulk upload of a significant number of monographic records from OCLC.

2 Increase reflects the purchase of a large electronic monographic package.

4.b Increase reflects improved counting methods and additional titles in packages of electronic journals.

11 Decrease reflects the weeding of materials in the Curriculum Materials Center.

15.c Includes maps, serials backfiles, spoken word files, manuscripts, films/videos, microforms, music, and software.

15.d Includes bibliographic utilities.

26.a Grant FTE for professional staff is 2.

26.c Grant FTE for student assistants is 1.

33 Change reflects a decrease of traffic in the building due to building renovation.

\section{SUNY-ALBANY}

1.b.i Includes 6,313 e-books.

$20 \$ 122,929$ of the total represents expenditures from private giving to the Libraries.

33 2004-05 figure revised to 202,009. 


\section{SUNY-BUFFALO}

9 Total was revised in 2005-06. No updates had been provided in the past several years.

39-42 Enrollment data submitted in 2004-05 was for the incorrect academic year. 2004-05 figures revised to (39) 22,468; $(40)$ 4,$808 ;(41)$ 5,959; (42) 3,479.

\section{SUNY-STONY BROOK}

Volumes held June 30, 2005 revised to 2,215,589 to include 12,580 electronic books.

15 Includes research and foundation funds and NYS Coordinated Collection Development Grant (\$101,402).

15.d Includes bibliographic utilities $(\$ 289,790)$, memberships $(\$ 93,315)$, ILL and document delivery $(\$ 44,000)$, SUNYConnect fee $(\$ 79,427)$, digitization fees $(\$ 21,517)$, off-site storage $(\$ 21,941)$, freight $(\$ 41,205)$, gifts program $(\$ 16,185)$, processing supplies $(\$ 29,623)$.

16 Includes money from NYS preservation grant $(\$ 7,979)$.

37, 38 Figures are for Main Campus and Health Sciences Center.

\section{SYRACUSE}

4.b Government documents that are serials are counted here; MARCHIVE records may not provide accurate data.

15 2004-05 figure was incorrectly reported and should be revised to $\$ 4,545,616$.

15.c Library is able to pull out accurate data on AV and map expenditures; expenditures data able to be shifted here from Miscellaneous (15d).

15.d Includes Law Library only.

17.a Five positions are cost-share positions.

41, 42 Includes law school students.

TEMPLE

Volumes held June 30, 2005 revised to 3,016,464 to include another cataloged portion of the Arabic collection.

2 Increase reflects substantial increase in budget.

7 Due to completion of retrospective conversion, all government documents now cataloged.

\section{TENNESSEE}

Volumes held June 30, 2005 revised to 3,682,677 due to change in counting method, and due to the addition of 160,918 e-books from Early English Books Online and Eighteenth Century Collections Online.

4.b Removed 5,617 titles from the count, for Gale InfoTrac OneFile database which were incorrectly included in 2004-05 (consortial full-text articles). These titles are now included in the Supplementary Statistics.

23.b Cost is calculated from a percentage of searches done over the course of a year. Increase from 2004-05 is due to the use of a federated search engine (Powersearch).

TEXAS

2 Includes 58,006 monographic volumes purchased by the University Libraries, 158 by the Center for American History, 9,416 by the Tarlton Law Library, and 479 by the Humanities Research Center.

15.a Includes $\$ 4,890,215$ by the University Libraries, $\$ 5,825$ by the Center for American History, $\$ 215,388$ by the Tarlton Law Library, and $\$ 54,130$ by the Humanities Research Center.

15.b Includes $\$ 7,667,919$ by the University Libraries, $\$ 6,254$ by the Center for American History, $\$ 919,516$ by the Tarlton Law Library, and $\$ 16,596$ by the Humanities Research Center.

15.c Includes $\$ 294,943$ by the University Libraries, $\$ 0$ by the Center for American History, $\$ 348,036$ by the Tarlton Law Library, and $\$ 1,615,393$ by the Humanities Research Center.

15.d Includes $\$ 794$ by the University Libraries.

\section{TEXAS A\&M}

1 Increase beyond upper bound due to the purchase of a unique and sizeable eMonographs collection as well as an aggressive withdrawing project at one of TAMU Libraries' branches.

1.b.i Reflects the addition of a large eMonograph package purchase. 
1.b.ii Reflects withdrawing project conducted at Galveston Branch.

2 Figure reflects the purchase of large eMonograph collection.

4.a Figure reflects a change in the counting algorithm used and does not reflect an actual reduction in titles or resources. In previous years TAMU Libraries relied upon a combination of manual and computerized counts to obtain the Current Serials Purchased statistic. With the implementation of a new Serials Link Server(SFX) data are computed entirely through the link server. The result is the statistics will not only be more accurate but more consistent.

4.b Accurate physical count of free serials received through the Acquisitions Department as well as those received through Government Documents. In previous years this statistic was driven by sampling data.

7 Reflects pre-1976 government documents holdings.

31 Includes use of virtual reference (heavily marketed during 2005-06) and better tracking of reference transactions through the use of a Web-based tracker.

40 Figure reflects better tracking of this statistic.

\section{TEXAS TECH}

All figures are as of August 31, 2006.

15.d HSC figure includes: Amigos $(\$ 15,516)$; Amigos eReserve $(\$ 7,205)$; Amigos FirstSearch $(\$ 660)$; Cybertools $(\$ 26,340)$; ERIC Online (\$1,800); ExamMaster (\$2,000); First Consult (\$5,000); Gold Rush (\$3,600); Images MD (\$1,429); Info POEMS (\$6,138); Journal Citation Reports (\$4,227); MD Consult $(\$ 89,729) ;$ Natural Medicines $(\$ 1,416) ;$ OT Search (\$995); OVID (\$41,716); RefShare (\$583); TexShare (\$2,500); Databases/EbBSCO-Dyna Med $(\$ 10,000)$.

\section{TORONTO}

1-4 Includes monographic series and government documents.

15-25 Expenditures as reported in Canadian dollars: (15a) \$8,522,551; (15b) \$11,641,324; (15c) \$4,780,165; (15d) UA/NA; (15) $\$ 24,944,040$; (16) \$463,590; (17a) \$14,530,423; (17b) \$16,157,780; (17c) $\$ 3,991,028$; (17) $\$ 34,679,231$; (19) $\$ 6,908,106 ;(20)$ $\$ 66,994,967 ;$; (21) \$952,225; (22) \$3,618,224; (23a) \$1,317,779; (23b) UA/NA; (24) \$2,685,719; (25) \$43,714.

26.c In 2004-05 the number of casual staff was underreported by 13 and also excluded RCAT. In 2005-06 the number of casual staff includes RCAT.

TULANE

Volumes held June 30, 2005 revised to 3,486,823 as previous volume counts had omitted several large categories of materials including a large government documents collection.

1.b.ii Includes 12,600 music volumes and 428,001 government documents volumes destroyed in Hurricane Katrina.

6 Includes 9,519 microforms checked-in post-Katrina plus 18,269 reels of film returned from restoration.

7 Includes 1,378 checked-in post-Katrina plus 77,145 salvaged and returning from restoration.

36-38 Figures are same as 2004-05; new figures not available for 2005-06 due to interruption from Hurricane Katrina.

39-42 Figures are for spring 2006; data from fall 2005 unavailable due to Hurricane Katrina.

\section{UTAH}

15c, 15d Exclude Marriott Library; figures reflect sum of Law and Health Sciences.

\section{VANDERBILT}

1.a Volumes held June 30, 2005 revised to 3,215,163 to reflect e-books not included in previous years.

15.c Some expenditures included here were previously reported under Miscellaneous (15d).

21 Primarily for purchase of electronic journal backfiles.

38 This is the 2006-07 figure; 2005-06 data is not available.

\section{VIRGINIA}

4 Increase due to previously counted subscriptions that were cataloged and many subscriptions that were not in the catalog.

8 University Library figure for 2004-05 revised to 10,418.

17.c For the University Library, student figures include part-time wage and temp workers. 
23.b Figure for University Library for 2004-05 revised to $\$ 660,972$.

26.c For the University Library, student figures include part-time wage and temp workers; student FTE is an extrapolated number.

\section{VIRGINIA TECH}

15.c Expenditures are included in (15a) and (15b).

\section{WASHINGTON}

Changes in University of Washington statistics for 2005-06 also reflect addition of UW-Bothell and UW-Tacoma for the first time.

Volumes held June 30, 2005 revised to 7,011,833 to include 228,004 e-books added in 2005-06.

24, 25 Figures available only for Law Library and Medical Library.

26.a Includes librarians and "professional staff" category.

26.b No longer includes anyone from the "professional staff" category.

36 2004-05 figure revised to 530 .

\section{WASHINGTON STATE}

1.b.i Decrease due to 2004-05 figure counting e-books for the first time.

10 Increase due to counting regional campus holdings for the first time.

31 Estimate based in part on 2004-05 data.

32,33 Increase due in part to disproportionately large increases at regional campuses.

34, 35 Method of counting ILL transactions is not uniform across campuses.

\section{WASHINGTON U.-ST. LOUIS}

Volumes held June 30, 2005 revised to 3,891,802 to include 197,298 e-books not previously reported.

1.b.i Includes 793 e-books.

4.a Decrease represents continuation of project to cancel some journals in print that are also received electronically.

11 Reflects acquisition of new collection containing 48,480 photographs.

13 Represents acquisition of Bill Miles Collection of 20,254 film and video.

15.a Includes expenditures for electronic monographs previously reported under Miscellaneous (15d) and other library materials (15c).

15.d Able to allocate larger number of expenditures to monographs, serials and databases than in previous years, thus a decrease in charges previously posted as miscellaneous.

18 Fringes appear as Other Operating Expenditures (19) because they are included in the library budget.

19 Includes figures for utilities and rent, building a moritization for the renovation (which will be in budget for next 30 years) and fringes that are part of the library budget.

\section{WATERLOO}

1.b Figure reflects weeding and database clean-up projects undertaken in 2005-06.

2 Excludes e-books.

4 Includes 6,607 from print and microform; 13,917 from electronic.

15-25 Expenditures as reported in Canadian dollars: (15a) $\$ 1,265,684 ;(15 b) \$ 5,279,990 ;(15 c) \$ 299,449 ;(15 d) \$ 342,392 ;(15)$

$\$ 7,187,515 ;$; (16) $\$ 73,241$; (17a) $\$ 2,470,442$; (17b) $\$ 3,746,203$; (17c) $\$ 761,599 ;$; (17) $\$ 6,978,244 ;$ (19) $\$ 3,016,136$; (20)

\$17,255,136; (21) \$19,093; (22) \$4,077,983; (23a) \$0; (23b) \$0; (24) \$272,550; (25) \$92,186.

15.c Decrease relates to extraordinary one-time funding for electronic backfile purchases in 2004-05.

17 Salary and personnel figures are as of April 30, 2006.

19 Includes Gifts \& Endowments, which were not reported in previous years.

29, 30 Decrease in number of sessions, with significant increase in number of participants, is related to more focused 
instruction sessions, changes in scheduling, and literacy instruction for online courses.

\section{WAYNE STATE}

All figures are as of September 30, 2006 and include Reuther Archives of Labor and Urban Affairs.

Volumes held June 30, 2005 revised to 3,419,410.

4.a The serials count is taken from the online system. The system does not currently provide a count of purchased vs. not-purchased serials.

9 Figure is estimated.

11 Figure is estimated.

19 Includes fringe benefits.

32, 33 The 2004-05 figures were overstated by 3,076.

36 Includes Ed.D. degrees.

\section{WESTERN ONTARIO}

Volumes held June 30, 2005 revised to 3,206,490 due to a mostly automated count and the addition of 280,871 e-books.

8 Western Libraries are no longer counting computer files separately.

10 Figure is from the Archives and Research Collections Centre only.

11 Figure is from the Archives and Research Collections Centre only; count is number of photographic negatives in the London Free Press Photographic Negative Collection.

12, 13 Figures are from the Music Library only.

15-25 Expenditures as reported in Canadian dollars: (15a) $\$ 1,953,311 ;(15 b) \$ 8,710,898 ;$ (15c) UA/NA; (15d) $\$ 4,997 ;(15)$ $\$ 10,669,206$; (16) \$126,424; (17a) \$4,088,520; (17b) \$3,632,756; (17c) $\$ 387,255 ;(17) \$ 8,108,531 ;(19) \$ 873,935 ;(20)$ $\$ 19,778,096$; (21) \$68,721; (22) \$6,320,180; (23a) \$126,265; (23b) \$0; (24) \$374,766; (25) \$0.

\section{WISCONSIN}

7 Change is due to an error in past reporting (reported pieces and volumes resulting in some double reporting).

16 Decrease reflects completion of consolidation and move of Ebling Health Science Library collections (multiple year project).

21 A significant number of electronic journal backfiles were purchased on one-time funding.

YALE

Volumes held June 30, 2005 revised to 12,123,037.

2, 4a-b 2004-05 figures incorrectly reported volumes purchased. 2005-06 figures, and all figures before 2004-05, reported titles purchased.

8 1,327 computer files were added in 2005-06; a cumulative figure will not be reported.

32, 33 Yale University Library figures include reserve circulations since they cannot be excluded; for 2005-06, figures exclude ILL circulations previously included in Circulation count.

36 These are the doctoral degrees taken from the IPEDS Survey of Degrees Granted. First Professional degrees are not included.

37 First Professional fields not included.

38 Number is full time ladder faculty at the University.

\section{YORK}

8 2004-05 figure revised to 2,889, identical to 2005-06 figure.

15-25 Expenditures as reported in Canadian dollars: (15a) $\$ 2,543,253$; (15b) $\$ 7,043,879 ;(15 c) \$ 163,170 ;$ (15d) UA/NA; (15) $\$ 9,750,302 ;$; 16 ) \$163,573; (17a) \$4,939,032; (17b) \$4,886,995; (17c) \$1,157,301; (17) \$10,983,328; (19) \$1,313,036; (20) \$22,210,239; (21) \$502,126; (22) \$4,760,582; (23a) \$71,012; (23b) \$0; (24) \$236,227; (25) \$10,228. 


\title{
APPENDIX A - ARL MEMber LibRARIES AS OF JANUARY 1, 2008
}

The Association of Research Libraries (ARL) represents the interests of 123 libraries that serve major North American research institutions. The ARL Statistics and Measurement program is organized around identifying, collecting, analyzing, and distributing quantifiable information describing the characteristics of research libraries.

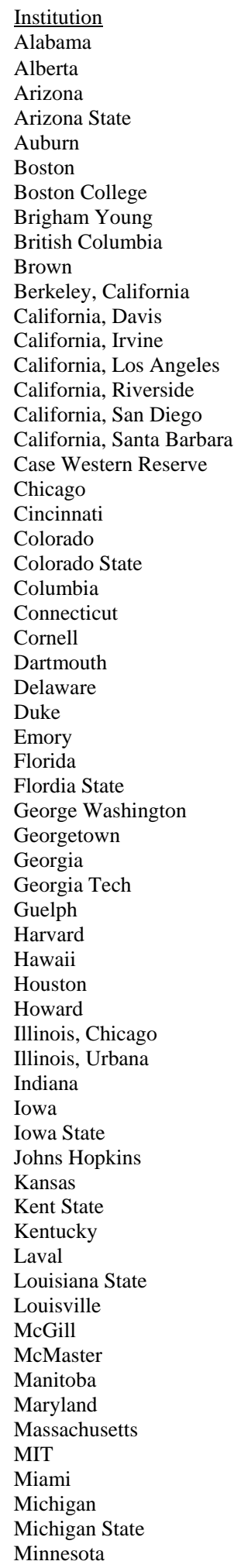

\begin{tabular}{|c|c|}
\hline Category & Full Name of Institution \\
\hline$S$ & $\overline{\text { University of Alabama }}$ \\
\hline $\mathrm{C}$ & University of Alberta \\
\hline S & University of Arizona \\
\hline S & Arizona State University \\
\hline S & Auburn University \\
\hline $\mathrm{P}$ & Boston University \\
\hline $\mathrm{P}$ & Boston College \\
\hline $\mathrm{P}$ & Brigham Young University \\
\hline $\mathrm{C}$ & University of British Columbia \\
\hline $\mathrm{P}$ & Brown University \\
\hline S & University of California, Berkeley \\
\hline S & University of California, Davis \\
\hline S & University of California, Irvine \\
\hline S & University of California, Los Angeles \\
\hline S & University of California, Riverside \\
\hline S & University of California, San Diego \\
\hline S & University of California, Santa Barbara \\
\hline $\mathrm{P}$ & Case Western Reserve University \\
\hline $\mathrm{P}$ & University of Chicago \\
\hline S & University of Cincinnati \\
\hline S & University of Colorado \\
\hline S & Colorado State University \\
\hline $\mathrm{P}$ & Columbia University \\
\hline S & University of Connecticut \\
\hline $\mathrm{P}$ & Cornell University \\
\hline $\mathrm{P}$ & Dartmouth College \\
\hline S & University of Delaware \\
\hline $\mathrm{P}$ & Duke University \\
\hline $\mathrm{P}$ & Emory University \\
\hline S & University of Florida \\
\hline S & Florida State University \\
\hline $\mathrm{P}$ & George Washington University \\
\hline $\mathrm{P}$ & Georgetown University \\
\hline S & University of Georgia \\
\hline S & Georgia Institute of Technology \\
\hline C & University of Guelph \\
\hline $\mathrm{P}$ & Harvard University \\
\hline S & University of Hawaii \\
\hline S & University of Houston \\
\hline $\mathrm{P}$ & Howard University \\
\hline S & University of Illinois at Chicago \\
\hline S & University of Illinois at Urbana \\
\hline S & Indiana University \\
\hline S & University of Iowa \\
\hline S & Iowa State University \\
\hline $\mathrm{P}$ & Johns Hopkins University \\
\hline S & University of Kansas \\
\hline S & Kent State University \\
\hline S & University of Kentucky \\
\hline C & Laval University \\
\hline S & Louisiana State University \\
\hline S & University of Louisville \\
\hline $\mathrm{C}$ & McGill University \\
\hline $\mathrm{C}$ & McMaster University \\
\hline $\mathrm{C}$ & University of Manitoba \\
\hline S & University of Maryland \\
\hline S & University of Massachusetts \\
\hline $\mathrm{P}$ & Massachusetts Institute of Technology \\
\hline $\mathrm{P}$ & University of Miami \\
\hline S & University of Michigan \\
\hline S & Michigan State University \\
\hline S & University of Minnesota \\
\hline
\end{tabular}

\author{
Location \\ Tuscaloosa, Alabama \\ Edmonton, Alberta \\ Tucson, Arizona \\ Tempe, Arizona \\ Auburn, Alabama \\ Boston, Massachusetts \\ Boston, Massachusetts \\ Provo, Utah \\ Vancouver, British Columbia \\ Providence, Rhode Island \\ California, Berkeley \\ Davis, California \\ Irvine, California \\ Los Angeles, California \\ Riverside, California \\ La Jolla, California \\ Santa Barbara, California \\ Cleveland, Ohio \\ Chicago, Illinois \\ Cincinnati, Ohio \\ Boulder, Colorado \\ Fort Collins, Colorado \\ New York, New York \\ Storrs, Connecticut \\ Ithaca, New York \\ Hanover, New Hampshire \\ Newark, Delaware \\ Durham, North Carolina \\ Atlanta, Georgia \\ Gainesville, Florida \\ Tallahassee, Florida \\ Washington, D.C. \\ Washington, D.C. \\ Athens, Georgia \\ Atlanta, Georgia \\ Guelph, Ontario \\ Cambridge, Massachusetts \\ Honolulu, Hawaii \\ Houston, Texas \\ Washington, D.C. \\ Chicago, Illinois \\ Urbana, Illinois \\ Bloomington, Indiana \\ Iowa City, Iowa \\ Ames, Iowa \\ Baltimore, Maryland \\ Lawrence, Kansas \\ Kent, Ohio \\ Lexington, Kentucky \\ Quebec, Quebec \\ Baton Rouge, Louisiana \\ Louisville, Kentucky \\ Montreal, Quebec \\ Hamilton, Ontario \\ Winnipeg, Manitoba \\ College Park, Maryland \\ Amherst, Massachusetts \\ Cambridge, Massachusetts \\ Coral Gables, Florida \\ Ann Arbor, Michigan \\ East Lansing, Michigan \\ Minneapolis, Minnesota
}

S: U.S. public univ.; P: U.S. private univ.; C: Canadian univ.; N: U.S. non-univ.; X: Canadian non-univ. 


\begin{tabular}{|c|c|c|}
\hline Institution & Category & Full Name of Institution \\
\hline$\overline{\text { Missouri }}$ & $\mathrm{S}$ & University of Missouri \\
\hline Montreal & $\mathrm{C}$ & University of Montreal \\
\hline Nebraska & $\mathrm{S}$ & University of Nebraska-Lincoln \\
\hline New Mexico & $\mathrm{S}$ & University of New Mexico \\
\hline New York & $\mathrm{P}$ & New York University \\
\hline North Carolina & $\mathrm{S}$ & University of North Carolina \\
\hline North Carolina State & $\mathrm{S}$ & North Carolina State University \\
\hline Northwestern & $\mathrm{P}$ & Northwestern University \\
\hline Notre Dame & $\mathrm{P}$ & University of Notre Dame \\
\hline Ohio & $\mathrm{S}$ & Ohio University \\
\hline Ohio State & $\mathrm{S}$ & Ohio State University \\
\hline Oklahoma & $\mathrm{S}$ & University of Oklahoma \\
\hline Oklahoma State & $\mathrm{S}$ & Oklahoma State University \\
\hline Oregon & $\mathrm{S}$ & University of Oregon \\
\hline Pennsylvania & $\mathrm{P}$ & University of Pennsylvania \\
\hline Pennsylvania State & $\mathrm{S}$ & Pennsylvania State University \\
\hline Pittsburgh & $\mathrm{S}$ & University of Pittsburgh \\
\hline Princeton & $\mathrm{P}$ & Princeton University \\
\hline Purdue & $\mathrm{S}$ & Purdue University \\
\hline Queen’s & $\mathrm{C}$ & Queen's University \\
\hline Rice & $\mathrm{P}$ & Rice University \\
\hline Rochester & $\mathrm{P}$ & University of Rochester \\
\hline Rutgers & $\mathrm{S}$ & Rutgers University \\
\hline Saskatchewan & $\mathrm{C}$ & University of Saskatchewan \\
\hline South Carolina & $\mathrm{S}$ & University of South Carolina \\
\hline Southern California & $\mathrm{P}$ & University of Southern California \\
\hline Southern Illinois & $\mathrm{S}$ & Southern Illinois University \\
\hline SUNY-Albany & $\mathrm{S}$ & University at Albany, State University of New York \\
\hline SUNY-Buffalo & $\mathrm{S}$ & University at Buffalo, State University of New York \\
\hline SUNY-Stony Brook & $\mathrm{S}$ & State University of New York at Stony Brook \\
\hline Syracuse & $\mathrm{P}$ & Syracuse University \\
\hline Temple & $\mathrm{S}$ & Temple University \\
\hline Tennessee & $\mathrm{S}$ & University of Tennessee \\
\hline Texas & $\mathrm{S}$ & University of Texas \\
\hline Texas A\&M & $\mathrm{S}$ & Texas A\&M University \\
\hline Texas Tech & $\mathrm{S}$ & Texas Tech University \\
\hline Toronto & $\mathrm{C}$ & University of Toronto \\
\hline Tulane & $\mathrm{P}$ & Tulane University \\
\hline Utah & $\mathrm{S}$ & University of Utah \\
\hline Vanderbilt & $\mathrm{P}$ & Vanderbilt University \\
\hline Virginia & $\mathrm{S}$ & University of Virginia \\
\hline Virginia Tech & $\mathrm{S}$ & Virginia Polytechnic Institute \& State University \\
\hline Washington & $\mathrm{S}$ & University of Washington \\
\hline Washington State & $\mathrm{S}$ & Washington State University \\
\hline Washington U.-St. Louis & $\mathrm{P}$ & Washington University \\
\hline Waterloo & $\mathrm{C}$ & University of Waterloo \\
\hline Wayne State & $\mathrm{S}$ & Wayne State University \\
\hline Western Ontario & $\mathrm{C}$ & University of Western Ontario \\
\hline Wisconsin & $\mathrm{S}$ & University of Wisconsin \\
\hline Yale & $\mathrm{P}$ & Yale University \\
\hline York & $\mathrm{C}$ & York University \\
\hline Boston Public Library & $\mathrm{N}$ & Boston Public Library \\
\hline Canada Inst. SciTech Info. & $\mathrm{X}$ & Canada Inst. for Scientific \& Technical Information \\
\hline Center for Research Libs. & $\mathrm{N}$ & Center for Research Libraries \\
\hline Library of Congress & $\mathrm{N}$ & Library of Congress \\
\hline Natl. Agricultural Lib. & $\mathrm{N}$ & National Agricultural Library \\
\hline Lib. \& Archives of Canada & $\mathrm{X}$ & The Library and Archives of Canada \\
\hline Natl. Library of Medicine & $\mathrm{N}$ & National Library of Medicine \\
\hline New York Public Library & $\mathrm{N}$ & New York Public Library \\
\hline New York State Library & $\mathrm{N}$ & New York State Library \\
\hline Smithsonian Institution & $\mathrm{N}$ & Smithsonian Institution \\
\hline
\end{tabular}

Location

Columbia, Missouri

Montreal, Quebec

Lincoln, Nebraska

Albuquerque, New Mexico

New York, New York

Chapel Hill, North Carolina

Raleigh, North Carolina

Evanston, Illinois

Notre Dame, Indiana

Athens, Ohio

Columbus, Ohio

Norman, Oklahoma

Stillwater, Oklahoma

Eugene, Oregon

Philadelphia, Pennsylvania

University Park, Pennsylvania

Pittsburgh, Pennsylvania

Princeton, New Jersey

West Lafayette, Indiana

Kingston, Ontario

Houston, Texas

Rochester, New York

New Brunswick, New Jersey

Saskatoon, Saskatchewan

Columbia, South Carolina

Los Angeles, California

Carbondale, Illinois

Albany, New York

Buffalo, New York

Stony Brook, New York

Syracuse, New York

Philadelphia, Pennsylvania

Knoxville, Tennessee

Austin, Texas

College Station, Texas

Lubbock, Texas

Toronto, Ontario

New Orleans, Louisiana

Salt Lake City, Utah

Nashville, Tennessee

Charlottesville, Virginia

Blacksburg, Virginia

Seattle, Washington

Pullman, Washington

St. Louis, Missouri

Waterloo, Ontario

Detroit, Michigan

London, Ontario

Madison, Wisconsin

New Haven, Connecticut

North York, Ontario

Boston, Massachusetts

Ottawa, Ontario

Chicago, Illinois

Washington, D.C.

Beltsville, Maryland

Ottawa, Ontario

Bethesda, Maryland

New York, New York

Albany, New York

Washington, D.C. 


\section{APPENDIX B - BIBLIOGRAPHY}

\section{PART A: Selected Articles from the ARL Newsletter}

“The ARL Membership Criteria Index.” ARL: A Bimonthly Newsletter of Research Library Issues and Actions 197 (April 1998$)$ : 9.

"Assessing ILL/DD Services: New Cost-Effective Alternatives." ARL: A Bimonthly Report on Research Library Issues and Actions from ARL, CNI, and SPARC 236 (October 2004): 9.

Askew Waller, Consuella, and Kaylyn Hipps. “Using LibQUAL+ and Developing a Culture of Assessment in Libraries." ARL: A Bimonthly Report on Research Library Issues and Actions from ARL, CNI, and SPARC 221 (April 2002): 10-11.

Case, Mary M. "A Snapshot in Time: ARL Libraries and Electronic Journal Resources." ARL: A Bimonthly Newsletter of Research Library Issues and Actions 235 (August 2004): 1-10.

"The Impact of Serial Costs on Library Collections." ARL: A Bimonthly Report on Research Library Issues and Actions from ARL, CNI, and SPARC 218 (October 2001): 9.

Case, Mary M. and Judith Matz. "Framing the Issue: Open Access." ARL: A Bimonthly Report on Research Library Issues and Actions from ARL, CNI, and SPARC 226 (February 2003): 8-10.

Case, Mary M. and Prudence Adler. "Promoting Open Access." ARL: A Bimonthly Report on Research Library Issues and Actions from ARL, CNI, and SPARC 220 (February 2002): 1-5.

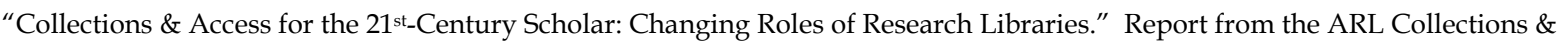
Access Issues Task Force. ARL: A Bimonthly Report on Research Library Issues and Actions from ARL, CNI, and SPARC 225 (December 2002).

Cook, Colleen, Fred Heath, and Bruce Thompson. "A Brief LibQUAL+ Phase One Progress Report." ARL: A Bimonthly Report on Research Library Issues and Actions from ARL, CNI, and SPARC 219 (December 2001): 7.

Crowe, William. "The End of History? Reflections on a Decade." ARL: A Bimonthly Report on Research Library Issues and Actions from ARL, CNI, and SPARC 226 (February 2003): 12-13.

Crow, Raym. "The Case for Institutional Repositories: A SPARC Position Paper." ARL: A Bimonthly Report on Research Library Issues and Actions from ARL, CNI, and SPARC 223 (August 2002): 1-4.

"The Future of Human Resources in Canadian Libraries." ARL, no. 240 (June 2005): 9.

Grosetta Nardini, Holly. "Building a Culture of Assessment." ARL: A Bimonthly Report on Research Library Issues and Actions from ARL, CNI, and SPARC 218 (October 2001): 11.

Groves, Richard. "Sharing Best Practices by Disseminating Assessment Results via the Web." ARL: A Bimonthly Report on Research Library Issues and Actions from ARL, CNI, and SPARC 236 (October 2004): 6.

Guedon, Jean-Claude. "Beyond Core Journals and Licenses: The Paths to Reform Scientific Publishing." ARL: A Bimonthly Report on Research Library Issues and Actions from ARL, CNI, and SPARC 218 (October 2001): 1-8.

Hahn, Karla. "The State of the Large Publisher Bundle: Findings from an ARL Member Survey." ARL: A Bimonthly Report on Research Library Issues and Actions from ARL, CNI, and SPARC 245 (April 2006).

Hipps, Kaylyn. "Diversity in the U.S. ARL Library Workforce." ARL: A Bimonthly Report on Research Library Issues and Actions from ARL, CNI, and SPARC 246 (June 2006): 1-2.

Johnson, Richard K. “Whither Competition?" ARL: A Bimonthly Report on Research Library Issues and Actions from ARL, CNI, and SPARC 217 (August 2001): 12-14.

Kyrillidou, Martha. "Reshaping ARL Statistics to Capture the New Environment." ARL: A Bimonthly Report on Research Library Issues and Actions from ARL, CNI, and SPARC 256 (February 2008): 9-11.

"The Impact of Electronic Publishing on Tracking Research Library Investments in Serials." ARL: A Bimonthly Report on Research Library Issues and Actions from ARL, CNI, and SPARC 249 (December 2006): 6-7. 
"The Future of Librarians in the U.S. Workforce." ARL: A Bimonthly Report on Research Library Issues and Actions from ARL, CNI, and SPARC 246 (June 2006): 5.

. "ARL University Libraries' Spending Trends." ARL: A Bimonthly Report on Research Library Issues and Actions from ARL, CNI, and SPARC 242 (October 2005): 10.

. "LibQUAL+TM in 2004." ARL: A Bimonthly Report on Research Library Issues and Actions from ARL, CNI, and SPARC 236 (October 2004): 6.

. "Serials Trends Reflected in the ARL Statistics 2002-03." ARL: A Bimonthly Report on Research Library Issues and Actions from ARL, CNI, and SPARC 234 (June 2004): 14-15.

Kyrillidou, Martha, and William Crowe. "In search of new measures." ARL: A Bimonthly Newsletter of Research and Library Issues and Actions 197 (April 1998): 8-10.

Kyrillidou, Martha, and Kaylyn Hipps. "Symposium on Measuring Library Service Quality." ARL: A Bimonthly Report on Research Library Issues and Actions from ARL, CNI, and SPARC 215 (April 2001): 9-11.

Lippincott, Sarah, and Martha Kyrillidou. "How ARL University Communities Access Information: Highlights From LibQUAL+TM." ARL: A Bimonthly Newsletter of Research Library Issues and Actions 236 (October 2004): 7-8.

Lynch, Clifford. "Institutional Repositories: Essential Infrastructure for Scholarship in the Digital Age." ARL: A Bimonthly Report on Research Library Issues and Actions from ARL, CNI, and SPARC 226 (February 2003): 1-7.

"Making Library Assessment Work." ARL: A Bimonthly Report on Research Library Issues and Actions from ARL, CNI, and SPARC 240 (June 2005): 9.

Shim, Wonsik "Jeff", Charles McClure, and John Carlo Bertot. "Measures and Statistics for Research Library Networked Services: ARL E-Metrics Phase II Report." ARL: A Bimonthly Report on Research Library Issues and Actions from ARL, CNI, and SPARC 219 (December 2001): 8-9.

Stuber, Peter. "Where does the Free Online Scholarship Movement Stand Today?" ARL: A Bimonthly Report on Research Library Issues and Actions from ARL, CNI, and SPARC 220 (February 2002): 9-15.

Unsworth, John M. "The Crisis in Scholarly Publishing in the Humanities." ARL: A Bimonthly Report on Research Library Issues and Actions from ARL, CNI, and SPARC 228 (June 2003): 1-4.

Wetzel, Karen, and Mary Jackson. "Portal Functionality Provided by ARL Libraries: Results of an ARL Survey." ARL: A Bimonthly Report on Research Library Issues and Actions from ARL, CNI, and SPARC 222 (June 2002): 7-9.

Wilder, Stanley. "New Hires in Research Libraries: Demographic Trends and Hiring Priorities." ARL: A Bimonthly Report on Research Library Issues and Actions from ARL, CNI, and SPARC 221 (April 2002): 5-8.

Young, Mark. "ARL Salary Survey Highlights." ARL: A Bimonthly Report on Research Library Issues and Actions from ARL, CNI, and SPARC 246 (June 2006): 4.

PART B: Related Books and Articles Published in Other Sources

Arms, William. "Quality Control in Scholarly Publishing on the Web." The Journal of Electronic Publishing 8, no. 1 (August 2002).

Baumol, W. J., and M. Marcus. Economics of Academic Libraries. Washington, D.C.: American Council of Education, 1973.

Bertot, John Carlo, Charles McClure, and Joe Ryan. Statistics and Performance Measures for Public Library Networked Services. Chicago: American Library Association, 2001.

Blixrud, Julia. "The Association of Research Libraries Statistics and Measurement Program: From Descriptive Data to Performance Measures." Proceedings from the $4^{\text {th }}$ Northumbria International Conference on Performance Measurement in Libraries and Information Services, edited by Joan Stein, Martha Kyrillidou, and Denise Davis. Washington, D.C.: Association of Research Libraries, 2002.

Carlson, Scott. “The Deserted Library: As Students Work Online, Reading Rooms Empty Out - Leading Some Campuses to Add Starbucks." The Chronicle of Higher Education, November 16, 2001.

Clapp, V. W. The Future of the Research Library. Urbana: University of Illinois Press, 1964. 
Coffman, Steve. “Building Earth's Largest Library: Driving into the Future.” Searcher 7, no. 3 (March 1999).

Competition Commission of the United Kingdom. Reed Elsevier plc and Harcourt General, Inc.: A report on the proposed merger, presented to Parliament by the Secretary of State and Trade and Industry by Command of Her Majesty, July 2001.

Cook, Colleen, Fred Heath, Martha Kyrillidou, and Duane Webster. “The Forging of Consensus: A Methodological Approach to Service Quality Assessment in Research Libraries - the LibQUAL+ Experience." Proceedings from the $4^{\text {th }}$ Northumbria International Conference on Performance Measurement in Libraries and Information Services, edited by Joan Stein, Martha Kyrillidou, and Denise Davis. Washington, D.C.: Association of Research Libraries, 2002.

Cook, Colleen, Fred Heath, and Bruce Thompson. "Score norms for improving library service quality: A LibQUAL+ study." portal: Libraries and the Academy 2 (2002): 13-26.

Cook, Colleen, Fred Heath, Bruce Thompson, and Duane Webster. “LibQUAL+TM Preliminary Results from 2002.” Performance Measurement and Metrics 4, no. 1 (2003): 38-47.

Cook, Colleen, Fred Heath, Bruce Thompson, and Russell Thompson. The Search for New Measures: The ARL LibQUAL+Project--A Preliminary Report. Baltimore: Johns Hopkins University Press, 2001. Accessed on the Internet at <http://muse.jhu.edu/demo/pla/1.1cook.html> on August 22, 2001.

"LibQUAL+: Service Quality Assessment in Research Libraries." IFLA Journal 27 (2001): 264-8.

Council on Library and Information Resources. A Different Approach to the Evaluation of Research Libraries. Research Brief 6. Washington, D.C.: The Council, 1998.

Cronenwett, Philip N., Kevin Osborn, Samuel A. Streit, eds. Celebrating Research: Rare and Special Collections from the Membership of the Association of Research Libraries. Washington, D.C.: ARL, 2007.

Crow, Raym. “The Case for Institutional Repositories: A SPARC Position Paper.” Washington, DC: Scholarly Publishing \& Academic Resources Coalition, 2002.

Cummings, Anthony M., et al. University Libraries and Scholarly Communication: A Study Prepared for The Andrew W. Mellon Foundation. Washington, D.C.: Association of Research Libraries, 1992.

Cummings, M. M. The Economics of Research Libraries. Washington, D.C.: Council on Library Resources, 1986.

Downs, Robert B. “The Growth of Research Collections.” Library Trends: American Library History: 1876-1976 25 (July 1976): 55-80. . Uniform Statistics for Library Holdings.” Library Quarterly 16 (January 1946): 63-69.

Drake, Miriam A. “Forecasting Academic Library Growth.” College and Research Libraries 37 (January 1976): 53-59.

Frazier, Kenneth. “The Librarians' Dilemma: Contemplating the Costs of the 'Big Deal,'” D-Lib Magazine 7, no. 3 (March 2001), viewed online at http://www.dlib.org/dlib/march01/frazier/03frazier.html.

Gooden, Paul, Matthew Owen, and Sarah Simon. Scientific Publishing: Knowledge is Power. New York: Morgan Stanley, 2002.

Hayes, Robert M. Strategic Management for Academic Libraries: A Handbook. Westport, Conn.: Greenwood Press, 1993.

Heath, Fred, Colleen Cook, Martha Kyrillidou, and Bruce Thompson. "ARL Index and other validity correlates of LibQUAL+ scores." portal: Libraries and the Academy 2 (2002): 27-42.

Hernon, Peter, and Robert E. Dugan. Action Plan for Outcomes Assessment in Your Library. Chicago: American Library Association, 2002.

Hernon, Peter, and John R. Whitman. Delivering Satisfaction and Service Quality. Chicago: American Library Association , 2001.

Hernon, Peter, and Ellen Altman. Assessing Service Quality. Chicago: American Library Association, 1998.

Hightower, Christy, Julie Sih, and Adam Tilgham. "Recommendations for Benchmarking Web Site Usage Among Academic Libraries." College and Research Libraries 59 (January 1998): 61-79.

Steve Hiller, Martha Kyrillidou and Jim Self, "Assessment in North American research libraries: a preliminary report card." Performance Measurement and Metrics 7 (2) (2006): 100-6. 
Jackson, Mary E. Measuring the Performance of Interlibrary Loan Operations in North American Research \& College Libraries. Washington, D.C.: Association of Research of Libraries, 1998.

Johnson, Richard K. “A Question of Access: SPARC, BioOne, and Society-Driven Electronic Publishing." D-Lib Magazine (May 2000), accessed on the Internet at <http://www.dlib.org/dlib/ may00/johnson/05johnson.html> on August 21, 2001.

Johnson, Richard K, and Judy Luther. “The E-Only Tipping Point for Journals.” Washington, D.C.: ARL, 2007.

Kling, Rob, Lisa Spector, and Geoff McKim. “The Guild Model.” The Journal of Electronic Publishing 8, no. 1 (August 2002).

Kuhlman, A. F. “Two ARL Approaches to Counting Holdings of Research Libraries.” College and Research Libraries 21 (May 1960): 207-11.

Kyrillidou, Martha. “Library Assessment as a collaborative enterprise.” Resource Sharing and Information Networks 1/2 (Special 200506 issue on the theme "Creative Collaborations: Libraries Within Their Institutions and Beyond"): 73-87.

Kyrillidou, Martha. "From Input and Output Measures to Quality and Outcome Measures, or, from the User in the Life of the Library to the Library in the Life of the User." The Journal of Academic Librarianship 28, no. 1 (January-March 2002): $42-46$.

"To Describe and Measure the Performance of North American Research Libraries." IFLA Journal 27 (2001, No. 4): 257-63.

"Research Library Trends: ARL Statistics." Washington, D.C.: Association of Research Libraries, 2001. Accessed on the Internet at <http://www.arl.org/stats/arlstat/jal99.html> on August 22, 2001.

Kyrillidou, Martha, and Fred Heath, eds. "Measuring Service Quality." Library Trends 49 (Spring 2001).

Lakos, Amos. "Culture of Assessment as a Catalyst for Organizational Culture Change in Libraries." Proceedings from the $4^{\text {th }}$ Northumbria International Conference on Performance Measurement in Libraries and Information Services, edited by Joan Stein, Martha Kyrillidou, and Denise Davis. Washington, D.C.: Association of Research Libraries, 2002.

Lavoie, Brian, Lynn Silipigni Connaway, and Lorcan Demsey. "Anatomy of aggregate collections: the example of Google Print for libraries" D-Lib Magazine (September 2005) 11 (9): http://www.dlib.org/dlib/september05/lavoie/09lavoie.html .

Lynch, Clifford A., and Joan K. Lippincott. "Institutional repository deployment in the United States as of early 2005" D-Lib Magazine (September 2005) 11 (9): http://www.dlib.org/dlib/september05/lynch/09lynch.html .

Mekkawi, Mod. “The ARL Library Index as a Decision-Making Tool.” College and Research Libraries 43 (September 1982): $396-401$.

Miller, Rush, and Sherrie Schmidt. "E-Metrics: Measures for Electronic Resources." Proceedings from the $4^{\text {th }}$ Northumbria International Conference on Performance Measurement in Libraries and Information Services, edited by Joan Stein, Martha Kyrillidou, and Denise Davis. Washington, D.C.: Association of Research Libraries, 2002.

Miller, Rush, Sherrie Schmidt, and Martha Kyrillidou. "New Initiatives in Performance Measures." Global Issues in $21^{\text {st }}$ Century Research Librarianship, edited by Sigrun Klara Hannesdottir. Helsinki: NORDINFO, 2002.

Molyneux, Robert E. “What Did Rider Do? An Inquiry into the Methodology of Fremont Rider's The Scholar and the Future of the Research Library." Libraries and Culture 29 (Summer 1994): 297-325.

. "Patterns, Processes of Growth, and the Projection of Library Size: A Critical Review of the Literature on Academic Library Growth." Library and Information Science Research 8 (January-March 1986): 5-28.

. The Gerould Statistics 1907/08 - 1961/62. Washington, D.C.: Association of Research Libraries, 1986.

Molyneux, Robert E., and Robert V. Williams. "Measuring the Internet." Annual Review of Information Science and Technology 34 (19992000): 287-339.

Morris, Dilys E., Collin B. Hobert, Lori Osmus, and Gregory Wool. “Cataloging Staff Costs Revisited.” LRTS 44 (2000): 70-83.

Nitecki, Danuta, and Toni Olshen. "Developing Service Quality Measurement Skills Through and Online Course for Librarians." Proceedings from the $4^{\text {th }}$ Northumbria International Conference on Performance Measurement in Libraries and Information Services, edited by Joan Stein, Martha Kyrillidou, and Denise Davis. Washington, D.C.: Association of Research Libraries, 2002.

O'Connor, Lisa, Carolyn Radcliff, and Julie Gedeon. "Applying Systems Design and Item Response Theory to the Problem of Measuring Information Literacy Skills." College and Research Libraries 63, no. 6 (2002): 528-543. 
Piternick, George. “ARL Statistics - Handle With Care." College and Research Libraries 38 (September 1977): 419-23.

“Library Growth and Academic Quality.” College and Research Libraries 24 (May 1963): 223-29.

Rider, Fremont. The Scholar and the Future of the Research Library. New York: Hadham Press, 1944.

Roche, Marilyn M. ARL/RLG Interlibrary Loan Cost Study. Washington, D.C.: Association of Research Libraries, 1993.

Rosenblatt, Susan. "Developing Performance Measures for Library Collections and Services." Chap. 18 in The Mirage of Continuity: Reconfiguring Academic Information Resources for the 21st Century, edited by Brian L. Hawkins and Patricia Battin. Washington, D.C.: Council on Library and Information Resources, 1998.

Shim, Wonsik, Charles McClure, and John Carlo Bertot. "Preliminary Statistics and Measures for ARL Libraries to Describe Electronic Resources and Services." Proceedings from the $4^{\text {th }}$ Northumbria International Conference on Performance Measurement in Libraries and Information Services, edited by Joan Stein, Martha Kyrillidou, and Denise Davis. Washington, D.C.: Association of Research Libraries, 2002.

Stubbs, Kendon. "Access and ARL membership criteria." Proceedings of the 125 th Meeting of the Association of Research Libraries (1993): $117-22$.

."University Libraries: Standards and Statistics." College and Research Libraries 42 (November 1981): 527-38.

“Apples and Oranges and ARL Statistics." Journal of Academic Librarianship 14 (September 1988): 231-35.

"On the ARL Library Index." Paper presented at the $108^{\text {th }}$ meeting of Research Libraries: Measurement, Management, Marketing (May 1986): Minneapolis, MN.

. "Lies, damned lies, ... and ARL Statistics?" Paper presented at the $108^{\text {th }}$ meeting of Research Libraries: Measurement, Management, Marketing (May 1986): Minneapolis, MN.

Thompson, Bruce. "Some Alternative Quantitative Library Activity Descriptions/Statistics that Supplement the ARL Logarithmic Index." Washington, D.C.: ARL, 2006.

Thompson, Bruce, Colleen Cook, and Russell Thompson. "Reliability and structure of LibQUAL+ scores." portal: Libraries and the Academy 2 (2002): 3-12.

van Westrienen, Gerard, and Clifford A. Lynch. “Academic Institutional Repositories: deployment status in 13 nations as of mid 2005" D-Lib Magazine (September 2005) 11 (9): http://www.dlib.org/dlib/september05/westrienen/09westrienen.html .

Weiner, Sharon. "Library quality and impact: Is there a relationship between new measures and traditional measures?" Journal of Academic Librarianship 31 (2005): 432-7.

Wilder, Stanley J. The Age Demographics of Academic Librarians: A Profession Apart. A Report Based on Data from the ARL Annual Salary Survey. Washington, D.C.: Association of Research Libraries, 1995.

Williams, Robert V. “The Making of Statistics of National Scope on American Libraries, 1836-1986: Purposes, Problems, and Issues.” Libraries and Culture 26 (Spring 1991): 464-85.

Wysocki, Bernard. “Scholarly Journals' Premiere Status is Diluted by Web." Wall Street Journal, May 23, 2005; Page A1. 